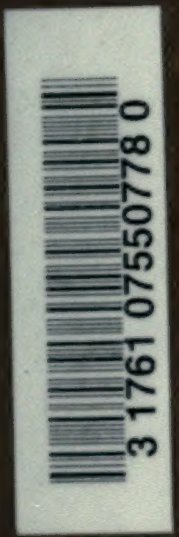


8198

\section{CH A S S E S}

DE L'ALGÉRIE 


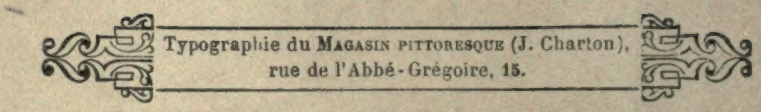




\title{
BIBLIOTHËQUE INSTRUCTIVE
}

\section{H A S S E S}

\author{
D E
}

L’A L GÉ R I E

ET NOTES

SUR LES ARABES DU SUD

$$
\text { PA R }
$$

Le Général A. MARGUERITTE

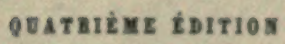

1L.LUSTHEE DE GS GRAYURES SUR BOIS

PARIS

\section{x. ฉ}

JOUVET ET C ie, EDITEURS

$$
\text { 5, MUE PALATINE, } 5
$$

\section{8}

Tous droilt rịervố. 


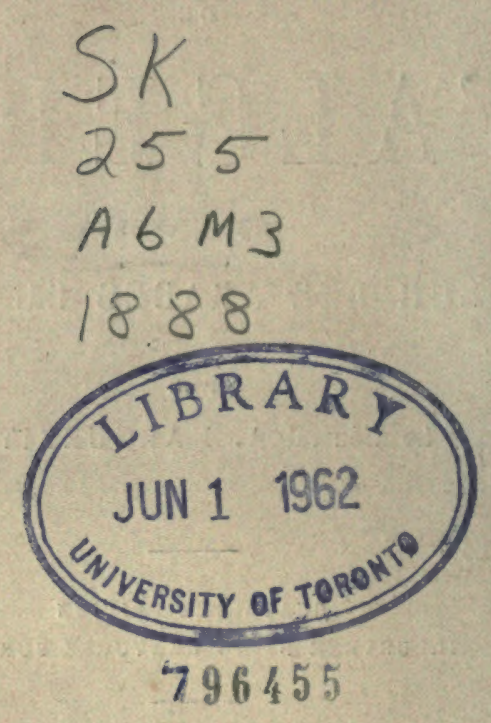




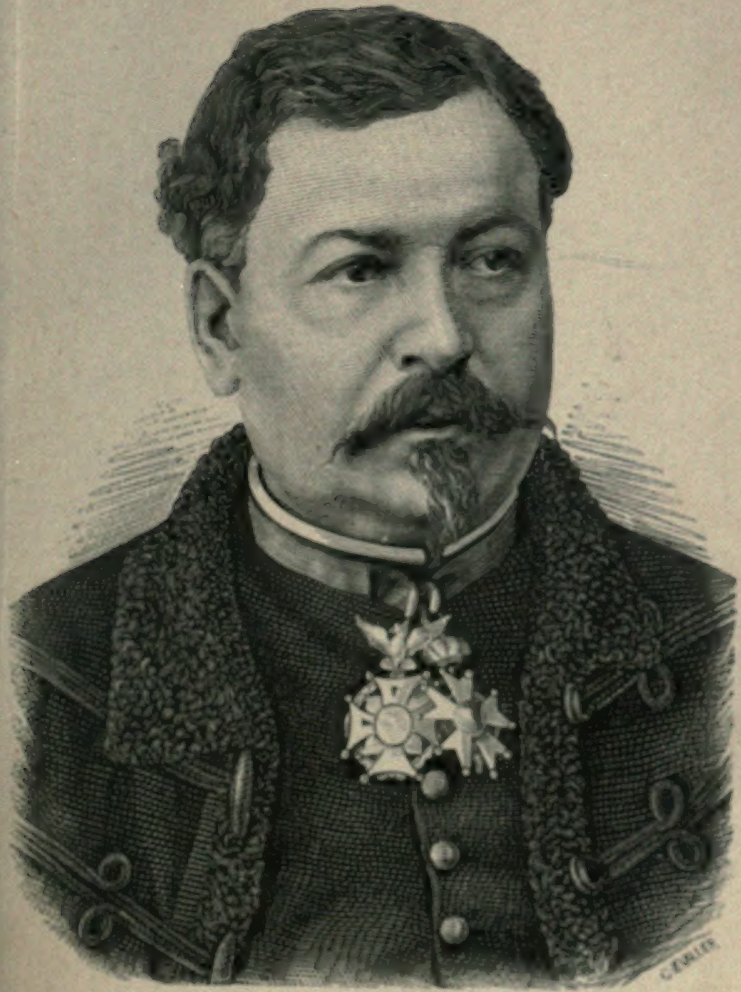

Le général A. Margueritte. 


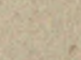




\section{DÉDICACE}

DF LA PRE:RIBHE BंDITIOY

lass souvenirs sont rloslines a mon fils, qui les lira quamb il sera en àge de les cumprendre el de sy intépissor.

a jen ai le sont et le loisir. je contimuerai les recilo d. une autres rhasses, qui aurout pour sujects:

I.as trous lions du Djelwet-R ilass:

las pautlicre de l'Uued-Masoine;

los rhasees aux sangliers:

La rhasse à liantilope bubalo:

las chasse aux mouflons:

leas explosits du vienx Monley, lo tuenor de lions des Blial, ine

liss recils auront, je limpoire, un terlain allrant pum

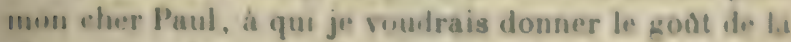

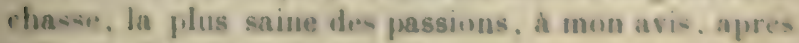
celles de l'élude el du travail.

1 Si tout arrive en ce momie, on peut dire aunat que tout siell va. Les Aralues d'anjourd'hus ne sont d.j. folus ceux d'il y a vingt ans: qpuelipue vingt anneses en

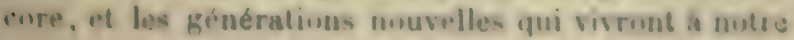




\section{DÉDICACE.}

contact ne présenteront plus lil mème originalité que celles observées au début de ma carrière en Algérie.

Quelques traits de leur caractère et de leurs aptitudes d'alors, pris sur le vif, ne seront pas sans intérêt pour Paul le Laghouati, qui aura à se rappeler qu’il est né dans le Sahara, où de bons Bédouins lui ont souhaité la bienvenue à son entrée dans la vie.

Si ces souhaits pouvaient avoir l’efłicacité de ceux des bonnes fées d'autrefois, mon petit Saharien serait merveilleusement doué; car il serait sage, savant, bon, brave, généreux, fort, cavalier parfait et chasseur intrépide, etc.

Moi aussi, cher enfant, je rous souhaite toutes ces vertus et brillantes qualités! - Mais, il faut bien l'avouer, c'est beaucoup désirer pour un seul, au temps présent!... Aussi prié-je votre mère de se joindre à moi pour m'aider à les demander à Dieu pour vous!

Quoi qu'il arrive, néammoins, et dès à présent, nous vous aimons bien.

A. Marguerit te.

Août 1866

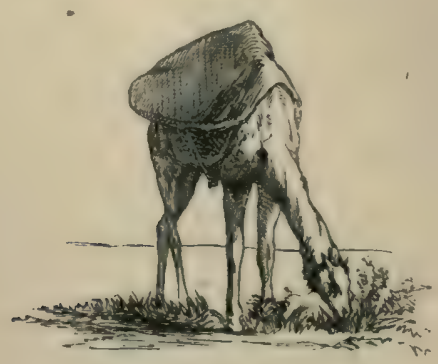




\section{PRE̊FACE}

DE LA IIE XIISAL LUITIOS

Jans le principr, es recits intimes nétaimt pas de-lines at las publicile.

1. insteur, par suite dune cireonstance foute partion-

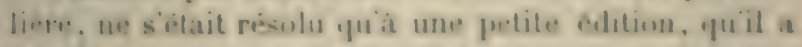
d.ifla a ses amis; il navait drautre pretention que

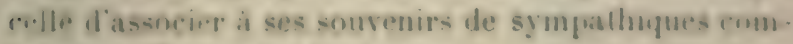

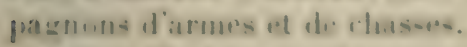

Anjourd hui encore il proserait de misme, sil naval

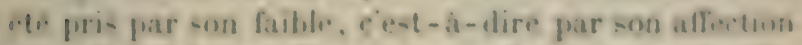

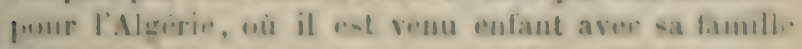
at an il a fait presigue loute sa carrite militare.

- Pourguor? Iui a-t-on dit, ne puthliez-sous fus ces

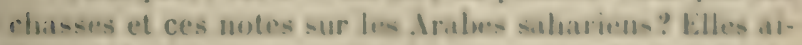
deront à melfre en reluef certaines partirularitios at

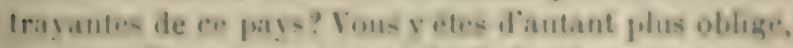

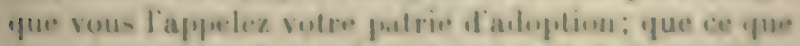
vous en dites est le resultiat de faits et diobservations

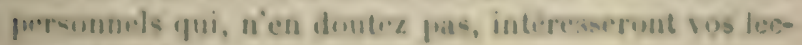
l.urs a l'Algérie ? $n$

Cirters, ronlit qui est siduisonul! 
Vos lecteurs! I Algérie! - l'est tout simplement magique, ces mots-là... De cette réflexion it la tentation de se faire imprimer vif, il n'y a qu'un pas..... et le voilà franchi!

Si vos prédictions ne se réalisent pas, chers conseitleurs qui maurez amené là, - vous aurez cet insnccès sur la conscience, car j'entends vous rendre solidaires de ma détermination.

Allez donc, rhers souvenirs, où votre destinée vous conduira. Dites surtout à ceux qui s'arrèteront à vous lire que l'AIgérie serail plus intimement pupulaire si elle était mieux connue. -- Dites-leur qu'elle possède un grand attrait d'originalité, un charme tout particulier qui agit infailliblement sur ceux qui s'y intéressent et savent la comprendre.

Insistez surtout près des disciples de saint IIubert, à quelque nationalité qu'ils appartiennent... Montrezleur dans un alléchant lointain ces rares et grandes bêtes à poursuivre... de bonnes émotions à glaner, lors même que le succès ne répondrait pas toujours aux espérances. - Et enfin, par anticipation, souhaitez à tous hienvenue et bonne chance!

Avril 1869.

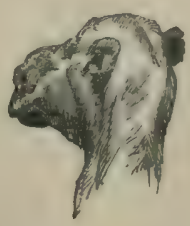




\section{LES CHASSES DE LALGERIE}

MA IREMIEIRE

\section{CIIASSE A U LION}

\section{Carcasconoe, noars 1868.}

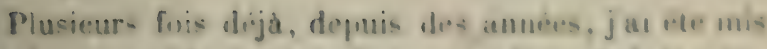
en demenre déerire les rhasees au linn et á la panlhere que jai fautes pendant mon long sijour dans le crivele de Téniet-el-Had.

Je un en suis toujours defendu, par paremed diaturd. ef auss je cros- par une surte dindillerence a narrer dere fails of des impressions dont je frouvais a ma ror lunte continuer la série.

Pendant une periode de vmyl-ciny anées, jas dio a ma destinie, qui ma toujours place anx asant-pustes it mesure que nous avancions dans Fintirieur du pays 
arabe, d'avoir la primeur de toutes les grandes chasses de l'Algérie ( $\left.{ }^{2}\right)$.

Quarais-je besoin decrire, quand en tous temps, it pied et it cheral, a la plaine et à la montagne, je pouvais faire plus de bourriches que je n'avais de gens à ıui les envoyer?

On réserve cela pour les tempis malheureux, quand frut gibier fait défaut. Hélas! ce n'est pas toujour: rète, selon le vieux dicton!

Aprè les grands festins, viemnent les jours maigres .t de pénitence; longtemps j'ai refusé d'y croire, mais depuis ma rentre sur le sol francais, jai dû me soumettre a l'évidence, cest-à-dire au régime du chonblane el du buisson creux.

Ir silis a l'heure présente ce que reut dire bredouille!

I es naturels du pays, excellentes gens du reste, ne parviernent à tirer un perdreau de loin eis loin queu firiant une neuvaine à saint Hubert.

Ma qualité d'hôte passager ne m’a pas mieux servi: qunique j'aie mis une grande persévérance ì battre les guiruts autour de notre bonne garnison de Carcassonne, je n'y ai vu qu'une seule fois l'ombre d'un lièvre.

C'est ainsi quapres de nombreuses déceptions ef n’avoir brûlé un peu de poudre qu'ì la chasse aux alouettes, j’ai vu venir la saison prohibée, toute hérissíc de gendarmes, de gardes champitres et autres!

(') C'est ainsi que j’ai pu claasser, dans les meilleures conditions, le lion, la panthère, le sanglier, l'hyène, le clacal, l'antilope bubale, le mouflon, la gazelle, le lynx, l'autruche, ete.; qque j’ai pu, avic d'excellents égruipage's de faucons, voler l'outarde, le lière, dans les plaines du Sud, et t'nlin chasser au marais des quantités considérables de sauragiaue à n'oser en the les chiffres. 


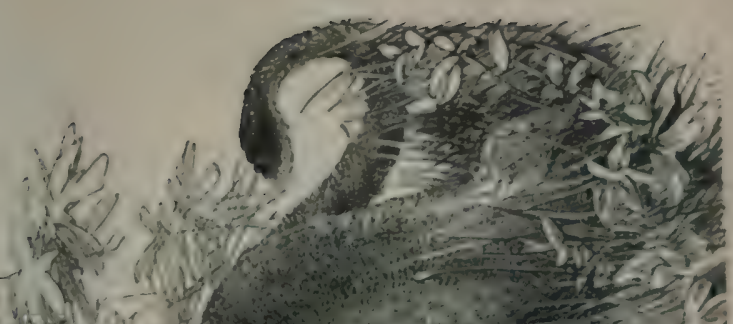

(2) 14 bow

(e)

q)

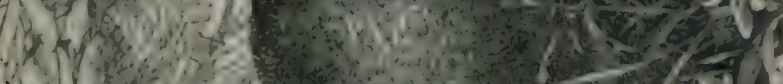

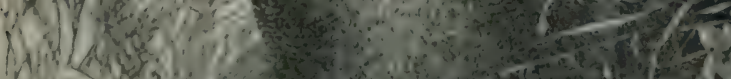

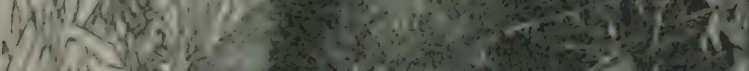

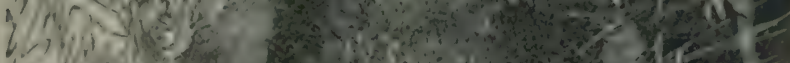

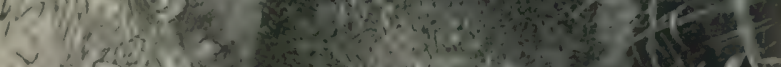

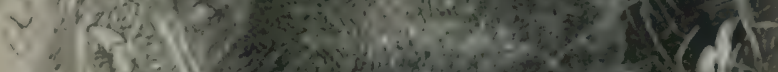

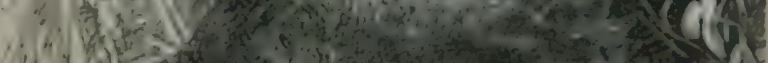

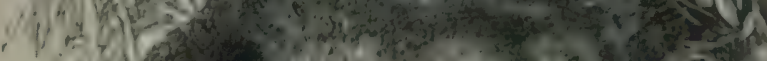

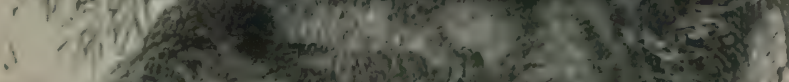

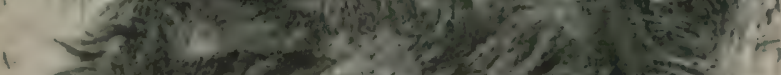

$\frac{8}{2}$

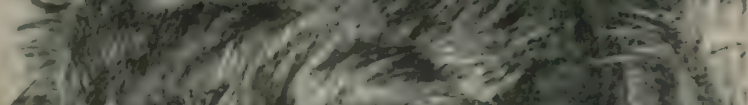
6) (1)

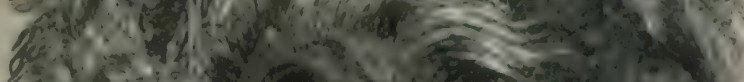

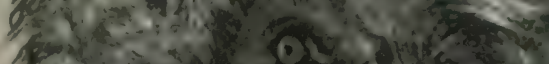

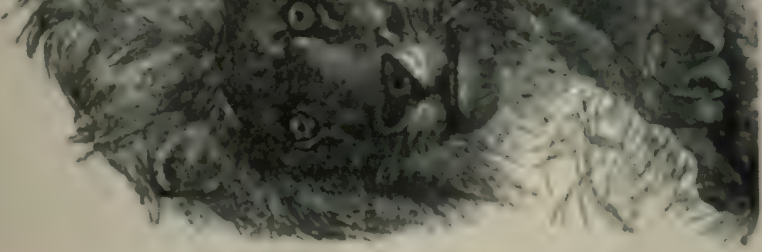



Uue faire en cetle extrémité? pécher à la lignue? I.e frujun lui-mème ne mord pas!

Allons, cette fois, il faut en prendre son parti, el. pruar tuer le temps à défaut de bêtes, je vais me raconter mes chasses diautrefois.

Cotte résolution me procurera, outre le placisir d. me rem morer de bunnes emotions, celui non muno grand the parler de quelyues-uns de mes compangums de chasse, braves Bedouins puur la plupart, que Limper aumat immortalises s'il arait pu les awnir peur types de ses livres, comme OEil-de-Faucon, Je? CerfArile. Chingagowk, etc.

II n' y aura dans mes récits aucune piripulie inventén apris coup. Leur morite, sils en ont, leur viendra de loner rigoureuse exactitude.

Cere-t, du reste, lessentiel quand le sujet trates est inlire-sant par lui-méme. Cerci dit et sals plus lon: preambule, j'entre en matiére.

La chase au lion est une des plus rulus que lim puisme taire, moins a cause des dangers a courir you par les dillicultes yue lion eprouve pour ahorder ce rou des animaux.

Míme dians les lowalitis quils fréquentent, les lison sont rares, quoi yqu ion en dise. Jai pasese onze ans dan. le pays de la prosince d'Alicer ai il $y$ en a le plus of jai constaté quili n'en a pas ate tué plus de truis ou ynatre en moyenne par annere.

Ciest dans la zone boiste (') et arcistentio prise entre

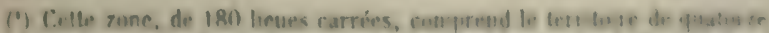

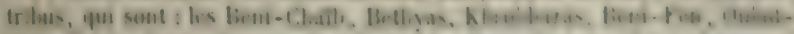

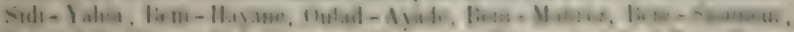

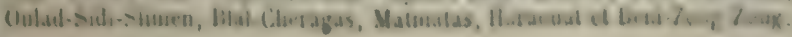


le Onarsenis à l'ouest, le pic de Taza à !'est. le DjebelEnnedate au sud et lia plaine du Chélif au nord, que se tiennent, pour le plus grand nombre, les lions et les panthères de la partie centrale de l’Algérie.

Dans cette grande superficie, vivent et se meuvent. autant que cela peut s'apprécier, une douzaine de lions: et panthères, y compris ceux que l'on désigne sou le nom de Berranis, étrangers, qui viennent accidentellement du Dir-Guezoul, dı Djebel-Dira et dı Zakkar.

La gent léonine est ainsi composée de deux tiers environ d'inligìnes, à demeure dans le pays, et d'un tier: de vagabonds.

Il faut croire que la reproduction de ces intéressanls animaux ne dépasse pas la destruction qui sen fail. car, au lieu d'augmenter, ils diminuent.

On peut prédire à coup sûr que, lans une vingtaine d'années, si on ne s'avise d'assurer la conservation de l'espèce par la défense de tuer les adultes au monent te la reproduction, il n'en restera que de bien rares. sprécimens, qui finiront même par disparaitre tout ì lait.

Lt ce serait grand dommage, à mon avis!

Ce noble roi des animaux, ce type du courage, de la force et de la magnanimité, ne doit pas disparaitre, comme un simple ruminant, d'un pars où nous l'avons encore à notre portée. Yon, il ne le faut pas! ne scraitce que pour conserver comme sujet de comparaison, et pour la plus grande émulation du genre humain, cette énergique et puissante bète.

. Yy aurait-il pas encore opportunité de ménager à nns arrière-neveux, sous prétexte de chasses et d'aventures périlleuses, quelques émotions propres à former 
unpape pour les organisations bataillmases et destruelives?

1. lisre ces riflexions at la sageses des penseur- de motre cpoxpue yui unt tant a faire, et roprends le fil de mon récit.

Cirs quelques lions, dissimines sur un si graml pays.

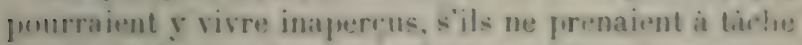
de se revéler par leurs dejpridations. Mais lis cun-mumition quids font, surtout en hiver. de beenfs, de va-

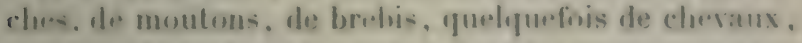
met bientut en emoi les tribus dont les troupeaus sunt summis a cette onereuse contrilution.

Il nest bruit alors que de borufo rasses, de monton:s

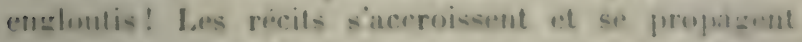

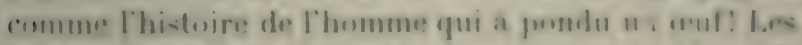

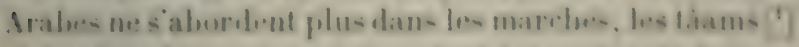

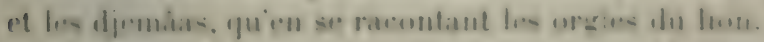

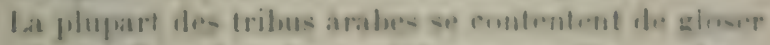

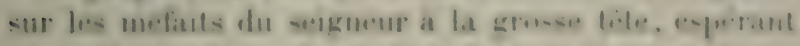

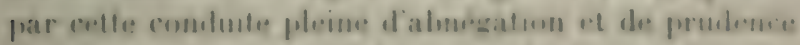

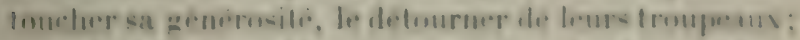

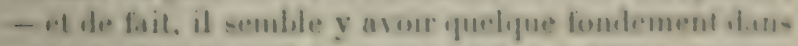

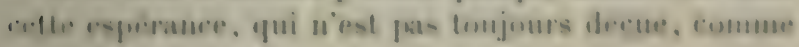
je le dirai plus tard.

bantres tribus, an contraure, quand le lion o montre

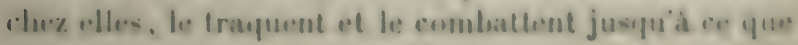
mort s'ensuive.

Jat tribu des Beni- Maluree e-t une de cos dimiores.

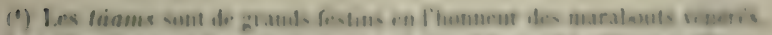

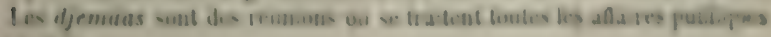
de lis titio. 
Depuis un temps immémorial, elle est en délicalesse avec les lions; sa situation en est la principale cause.

Placée entre le Djebel-Ennedate et Ic Djebel-R'ilass, les deux grands repaires de ce massif montagneux, les lions passent forcément sur son territoire en allant de l'un à l'autre, et se sustentent à ses dépens.

Jai fait un relevé de la moyenne des bestiaux mangés: en onze ans par les lions et panthires aux Beni-Mahrez: jai trouvé ces chiffres én moyenne par année:

Chevaux, juments ou poulains . Bœufs ou vaches.

Moutons ou brebis . . . . . . . 75

Soit une valeur de cinq mille francs environ, prélevée sur une tribu qui ne compte pas plus de cent tentes.

On peut dire généralement qu'il n'y a chez les Arabe: que les gens qui sont par trop agacés de la perte de leurs bestiaux qui combattent le lion.

Chez les Européens, c'est autre chose : ceux qui sont chasseurs sont entraînés par ce que l'on peut appeler la vocation de tout tuer.

C'est une monomanie, comme celle de certains naturalistes de tout collectionner.

Tant qu'il manque une peau à l'un, une planté иu un insecte à l'autre, leur bonheur n'est pas complet.

Le chassur veut pouvoir dire : J'ai tué de toute: les bêtes possibles!n

Le naturaliste veut pouvoir se glorifier que jas un échantillon des genres qu'il collectionne ne lui a échappé.

Et, pour alteindre ce résultat si désiré, tous denx sont capables de tout! 


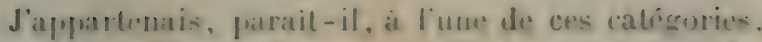
lorsyue je: fis, en 18\$4, nomme ehef du burean arabe de Téniet-el-lliul.

Javais eommene a chaserer at liage de douze ans: bracoup de bêtes drjà avaient blé me- victimes, maije n arais pas encore eu l'occasion de mattanuer ans Lions. The longue résidence a Téniet-el-Hà, siluée su le territoire des Beni-Mahrez, devait me la fournir.

Je nolais pas à mon poste depuis quinze jours, yue jentendais parler des lions, de leur proximiti, de l.mo ravages, etc.

l.hite que je pourrais en turer me vint alors ot mo demna un frisson de... joie, je pense; mais celte inlee to. put de sitot s'exécuter.

Três ucerupe, a mon debut, de l'organisation du pays. "t aus numbren-es expeditions dans le Ouarsenis, I.

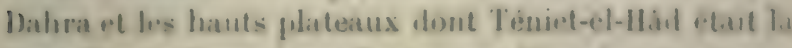
hose doprations, je ne fus un peu libre de mon temppue vire le commencement de 1816 .

fir-1 a celfe érenque que je me mis en relations asm un persounage impontant des Beni-Malırez, nomme R.iMokhlat-bel-Arthi: il etait frere de mon chamols e' alors caìd de sa tribu.

for-que je fis sa romaisance, il avuit deja tue quw. lore. lions ef trois pantheres.

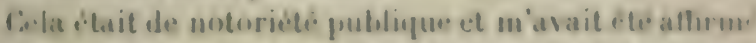

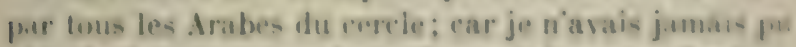
whtuir de ce trop modente Nemroul de me parler lon guement de ses chasses, de me dire le chiftre eacul d. seg victimes.

Uuand je le poussais trop sur ce puint, il tinissul te lencontre de cen qui a lieu dans les narrations de chan-

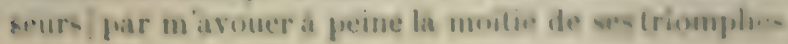


Il agissait ainsi, du reste, envers tous coux qui le metlaient sur ce sujet, et prétextait une afticire quelconque pour rompre l'entretien, si on faisait mine de le prolonger.

J'ai su depuis que la crainte du mauvais œil ( $\left.{ }^{1}\right)$ lui faisait employer ce subterfuge, ou cette extrème réserve, comme on voudra.

Quoi qu'il en soit, El-Mokhtar tuait proprement les lions et les panthères, tout seul.

Son procédé était très simple : il suivait leurs traces sur le sol détrempé par la pluie ou couvert de neige, il les abordait dans leurs repaires, et là, à la distance de quelques pas, il leur envoyait, arec un vieux fusil lont personne n'aurait donné dix francs, une balle si - ûre que la plupart du temps la bète ne sen relevait pas.

Je veux anticiper sur mes souvenirs pour raconter un Irait qui fera encore mieux connaitre cet intrépide Bédouin.

Un jour que nous avions suivi en vain, pendant toute ume journée, les traces d'une panthère, nous vimes celle-ci, à l'approche de la nuit, pénétrer dans une caverne sous un gros rocher, à une centaine de pas de nous.

Nous y allâmes aussitôt, et notre première idée fut le faire du feu à l'orifice de la carerne pour enfumer la bète et l'obliger à sortir.

Pendant que je tenais mon fusil braqué à l'ouverture,

(1) La crovance à l'influence du mauvais œil est très accréditée chez les Arabes. Ceux qui possèdent quelque chose d'enviable moralement ou physiquenent cherchent à s'en préserver en cachant ou en dissimulant le plus possibte ce qui peut être convoité par autrıi; l'usage vent, quand on a à lour' chez quelifu'un une qualite ou une possession quelconque, d'ajouter : "Que bieu bénisse et préserve du mauvais ocil? * 


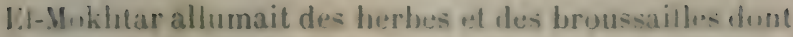

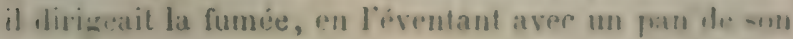

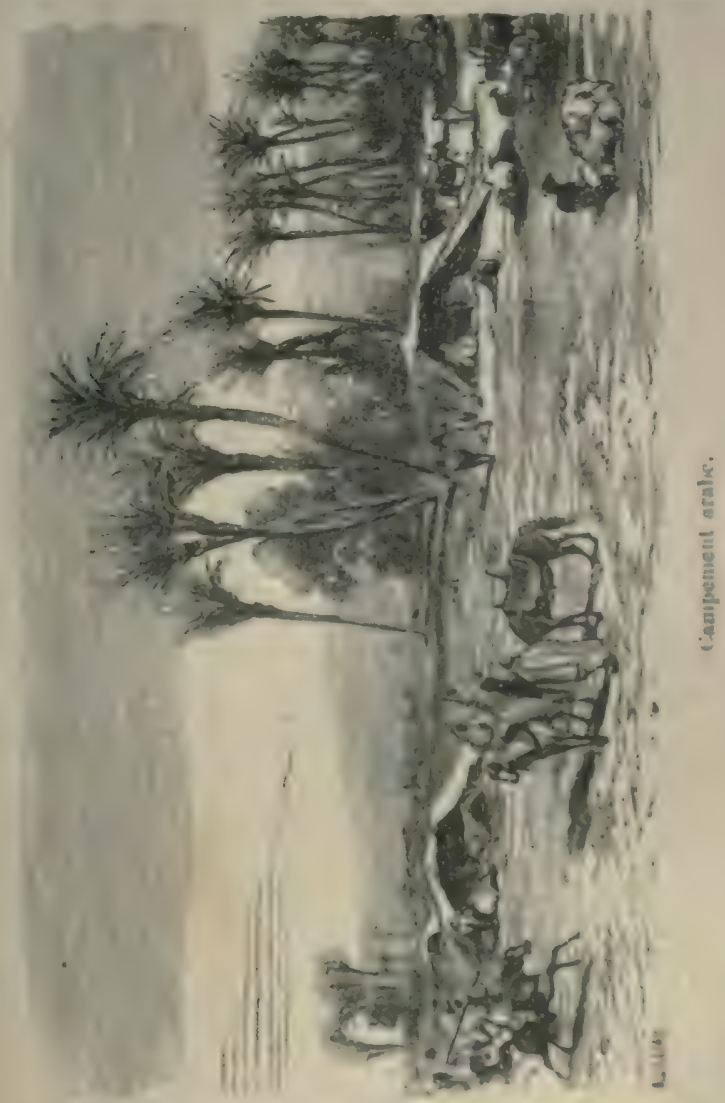

Burnons, dans linterieur du trou ou avait dinguru la jisuthere.

Apries une demi-lieure de ce m.un.ge que nous avons 
rmu devoir être efficace, la bète ne se déciclant pas ì surtir, j'allais proposer ì Li-Mokhtar de nous en aller. lorsque je le vis se défaire de ses vìtements, enrouler son burnous comme un manchon autour de son avantbras gauche, et, sa main droite armée de son couteau it raser, se mettre à plat ventre pour entrer dans lia cuverne.

-Que fais-tu? lui dis-je.

- Tu le vois bien!... Puisiuelle ne reut pas surtir. je vais aller lui couper le eou...

Et il se glissa la tèle la prenière dans l'entrée de lit caverne. Je l'appréhendai au corps et lui intimai d'Mi rester là de son entreprise, qui me paraissait insensée... J'eus toutes les peines du monde à l'empècher de lis mener jusqu'au bout.

"Je connais ce trou, me dit-il. Il est assez étroit pendant quelques pas, mais ensuite il s'élargit, on peut s'y tenir debout... Quand la panthère s'élanceri sur moi, je lui présenterai mon bras gauche, et pendant quille mordra dans le burnous, je lui ouvrirai te ventre arec mon couteau. J'ai fait cela sourent aves: des hyènes..."

Il l'eût fait encore comme il le disail et sans plus d'emphase, mais je ne voulus y consentir en ancun. ficcon. Finalement j'emmenai mon homme, qui ne se fit faute de maugréer d'aroir été arrèté dans une si belle aventure!

Je ne pouvais choisir, comme on le voit, un meilleur parrain pour faire mes premières armes en ce genre.

Un jour donc que El-Mokhtar m'apportait son troisième lion depuis mon arrivée à Téniet-el-Hàd, je lui fis la proposition d'aller avec lui pour essayer d'en luer aแssi. 
C.tte cuverture ne fut pas deburd bien accuedlie. II cralsuat quil ne marrivat mesaventure, et coin-ulerait comme bien grosse la responsabilite de the mettre aux prises avec de si rudes adversaires.

Veannoins, comme jinsistai beancoup. et hion con. vain:u, conme il le disait, que jitais maitre de men balle, il tinit par céder à mes instances.

Il avait justement ce jour-la connaissance d'un autre lion, sans doute frère de celıi quill arait tue la r.alle. leqfurel venait régulierenent chaque nuit, dequis une semaine, enlever des bribis daus un donar '/ gua eloigue du sien.

II etait probable yue ce lion ferait encore de neme la nuit prochane ${ }^{d}$ ) : nous purrions alors suise on traces, le terrain etant detremper, et le treer dans sum r.paire, si Dien en arait derute ainsi.

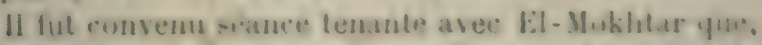

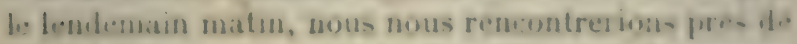
sa maison et que neus nous mettrions en chase ans. sitit, si les previsuns se realisaient.

Mon intentun aval ote daller seul aree lui, pour so conp dimsai; mais la nonvelle de mon projet setant reprandue, des ausilaures de lunne volonte se preasa. lirent pour se joindre a nous.

lip fut dabord min adjoint, M. le lientemant simziat, puis deux ofliciers du $2^{n}$ bataillon difrique, un de spahis, mon chawuih El-Midrouck, et cinj ou sis cavaliers indigènes dísirenx en rette circonstance do faire preuve de zele.

(1) Dowar, rëunaen de tentes alalus sormees en rond.

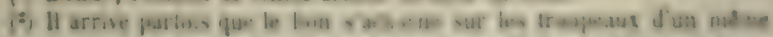

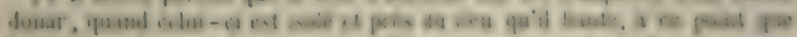

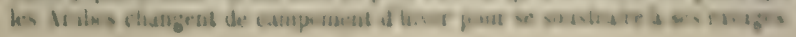


J'aurais eu mauvaise grâce de leur refuser d'assistur it cette chasse. Nous partimes donc le lendemain matin, au nombre d'une douzaine de tireurs et de quelques cavaliers pour tenir nos chevaux.

Nous trouvâmes lil-Mokhtar au reildez-vous, avec d'autres Arabes de ses administrés qui voulaient ausși suivre la chasse. Il nous donna la bonne nouvelle que le lion avait pris une brebis, selon son habitule, au douar qu'il soumetlait à rançon depuis huit jours, leyuel était peu distant de nous.

Il avait neigé la nuit, les pattes du lion étrient fortement empreintes sur le sol; elles mesuraient um grande main ouverte, ce qui indiquait une bète de la plus grande taille et nous donnait l'assurance de la pouvoir suivre.

El-Mokhtar aurait aussi préféré voir moins de mond. réuni pour cette expédition. Il savait par expérience 'que plus on est, moins on réussit, et que les accidents sont plus nombreux.

Il nous montra, à environ 1500 mètres, sur une pente boisée du Djebel-R'ilass, deux de ses parents qui suivaient lentement les traces ot les relevaient en altendant notre arrivée.

Nous allâmes à eux, et nous pùnes tous nous donner un avant-goût de la bète en contemplant les empreintes énormes qu'elle avait laissées sur la neige.

C'est vers deux heures du matin, nous dit-on, que le lion avait pénétré au milieu des tentes, en franchissant un abatis de branchages auquel elles étaient adossées.

Il lui avait fallu sauter au moins quatre mètres en hauteur sur dix de largeur pour pénétrer dans l'ellceinte! Il avait pris, en tombant comme la foudre ua 
mil:+u du troupeau ( ${ }^{1}$, qui s'y trouvait parque, une grusis brebis; puis, diun mème blan, avait franchi dr: numreau tentes et abalis.

Nayaut pas été inquiété dans sa retraite, il était nlle manger, a deux cents mètres do lat. la suadile lirehis, dont il n'avait laissé que qum-lupes flocons do I. iili.

Lil-Makhtar, tress au courant des habitul s dis lims qui fréquentent le pays, fut consulte sur les disprosiliums it prendre.

(9) On ne se figure gras le trouble et la confusion yua amine une parelloe scenr.

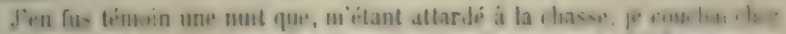

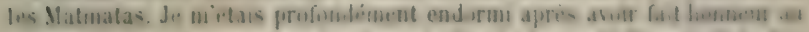

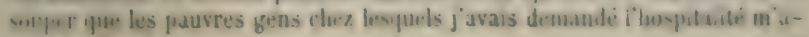
varetet oflert.

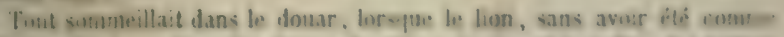

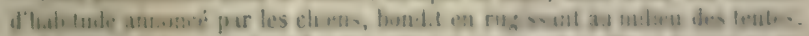

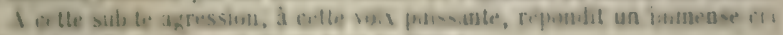

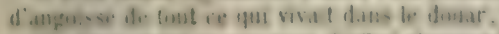

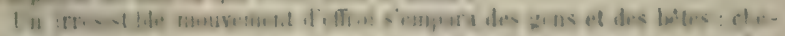

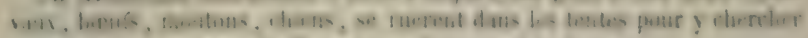

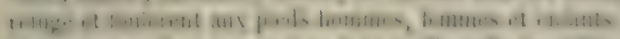

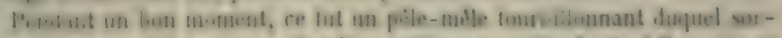

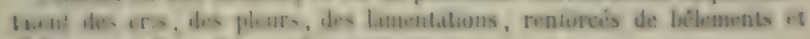

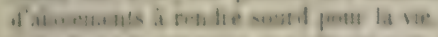

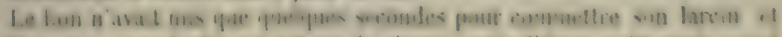

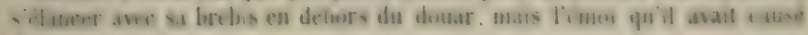
Aura jusqui iu jour

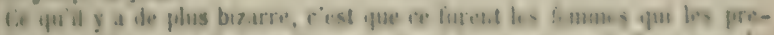

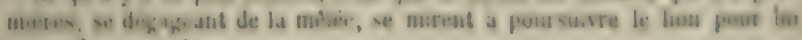
seprendre sa proie.

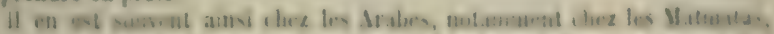

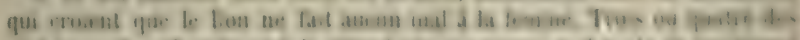

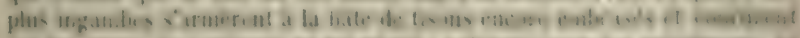

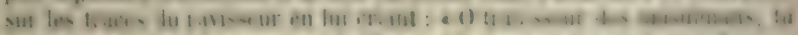

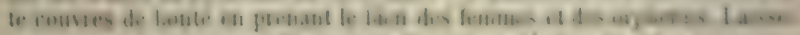

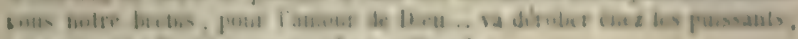

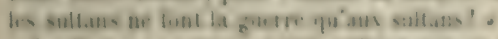

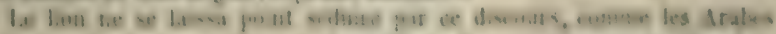

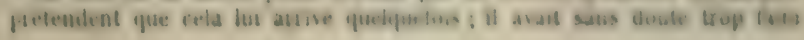


- "D'après la direction des traces, nous dit-il, et l'heure i laquelle le lion a mangé, il doit ètre allé cuire sa viande $\left({ }^{1}\right)$ dans le fourré des Saules ou du Rocher-du-Corbeau. - Je vais vous précéder de quelques centaines de pas, parce que vous faites trop de bruit avec vos cheraux... Je relìverai la piste avec soin, et quand j'aurai la certitude que le lion est dans l'un des fourrés, je vous ferai signe de mon burnous; vous mettrez alors pied à terre et me rejoindrez dans le plus grand silence. Nous aviserons ensuite. "

Les choses ainsi convenues, nous laissîmes ElMokhtar nous devancer, apris quoi nous reprimes notre marche.

Notre éclaireur se dirigea sur le fourré des Saules, où pénétraient les traces; il en fit le tour avec précaution, puis, les ayant retrouvées ì la sortie, il continuia sa marche lente et silenciense, que nous suivions it distance.

Nous nous dirigeâmes cette fois sur le repaire de

pour le quart d'heure, il emporta bel et bien la brebis et s'en fut la croquer à son aise dans le bois.

Les femmes revinrent exaspérées de leur insuccès.-Quant à moi, l'évinement m'avait surpris enveloppi dans mes burnous et un tapis; j'en fus quitte pour avoir été piétiné pendint deux ninutes par trois raches et leur progéniture.

Le lion rend la vie très dure aux gens dans le voisinage desquels il se cantonne, et, selon l'expression arahe, il leur enlève le sumneil des yeux.

Il est vrai qu'il n'attaque pas toujours, mais, par l'appréhiension qu'il donne de sa visite et par ses rugissements, il maintient les douars à deux ou truis lieces à la ronde dans un état d'anxiété fort pénible : les lommes veillent toute la nuit, en poussant de minute en minute des cris perçants et en jetant en l'air des tisons enflammés, quand les chiens, par leurs aboiements furibonds, annoncent l'approclie du lion.

(1) Cuire sa viande est l'expression usitée pour dire du lion qu'il fait sa digestion. Celle-ci est souvent pénible quand il a beaucoup mangé; il se tord, s'etire et gronde sourdement. l'est alors que les Arabes disent : Sbâ rah itebeulihr lagmou, - Le lion ruisine sa viande. 


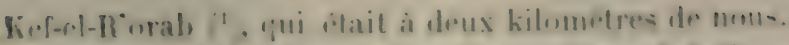

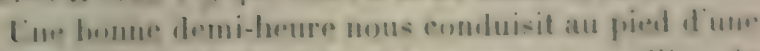

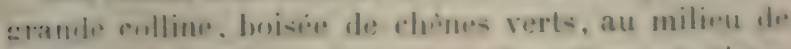
fapuelle sielevait un inorme reselere firmant plate-

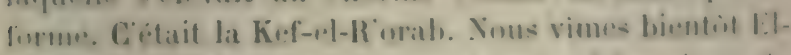

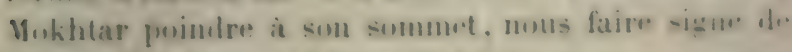
mellre poied at terre of des venir à lui.

Cuu-laissames nos rhevaux at la grarile de nos cava-

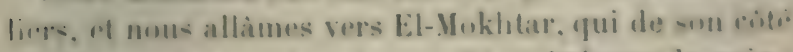
siut a motre reneontre et nous dit en bassant la vois:

"- I, linn est lit, dans lo groms huissun que vour

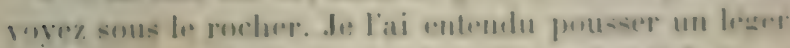
rugi-nement d'oveil, la viamle quil a mangere le lourmenter lates silenere. vous alle\% me suivere en marehaut fous dans mes pas, nous irous ain-i nems purers sur le.

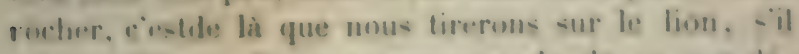
mel pussible. - Je vous rerommande de ne fas de-

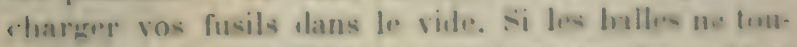

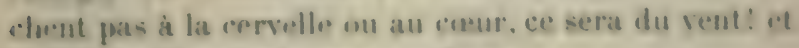

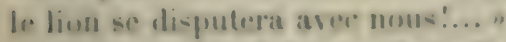

Irant dit, il prif la late de la tile: je marchais apres lui. suivi de mon adjoint et sucro:-sivement des autres lireurs.

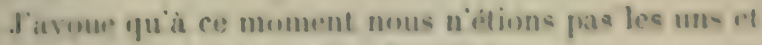
les autros sans une certatin emotion.

1.0 comblat allatil evidemmont s'enenger. Xims a a.

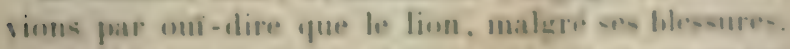

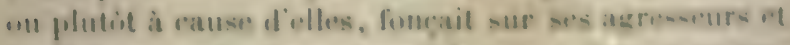

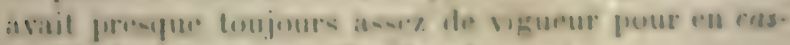
sep (2) plusieurs abaut diespirert...

(2) Rocluer-da - Corbean.

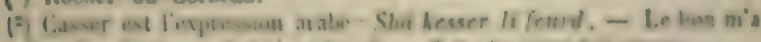

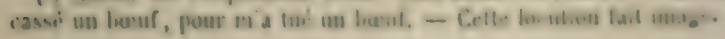


Nest-il pas vrai aussi qu'un genre de príril que $l^{\circ}(\mathrm{m}$ n'a pas encore affronté prend souvent dans limagination une importance plus grande que dans la réalité?... Donc le pouls nous battait plus vite. cela est certain.

Nos armes araient été chargées avec snin. J'avaijour mon compte une carabine de chasseurs a pied. du gros calibre. Elle était d'une justesse suffisante el je me promettais de ne m'en servir qu'à bon escient.

El-Mokhtar, après nous avoir fait exécuter un circuit pour donner moins d'éveil, nous conduisit sur le sommet de Kef-el-R'orab.

Cie rocher surplombait à pic, d'une hauteur de plus de quinze mètres, le fourré dans lequel était le lion: mais le bois en était si dru que, malgré l'élévation et notre vue plongeante, nous ne pouvions rien lécnuvrir.

I mesure que nous arrivions sur le rocher, nons nous placions les uns à côté des autres sur un rang. les fusils armés et prêts à meltre en joue.

Les deux tiers de notre froupe avaient déjà pris place de cette manière et sans faire de bruit, lorsqu'un des derniers Arabes, en marchant sur la partie déclive du rocher, glissa en arrière en laissant échapper sou fusil, qui rendit sur la pierre un son de ferraille.

I ce moment, le lion, qui sans doute nous voyait agir depuis quelques instants et n'attendait qu'un prétexte pour se révéler, répondit à ce bruit, qu'il prit jour un début d'hostilités, par un rugissement formiliable qui nous donna la chair de poule $\left({ }^{1}\right)$ !... En mème

(') Ou mieux, qui nous hérissa le poil. - J'ai éprouvé ce phénomène 'tui est un effet purement plịsique. Aussi, q'and je dis que nous eûmes la elair de poule, il ne fiut pais en conclure que nous eûmes peur, pour dire 
l.mpl il silanea vers nou- du milion do son fourre, en emoliant sous son elan dr jemurs chenes de la zrn-seur du bras, comme s'ils n'eussent eté que des roseatix!...

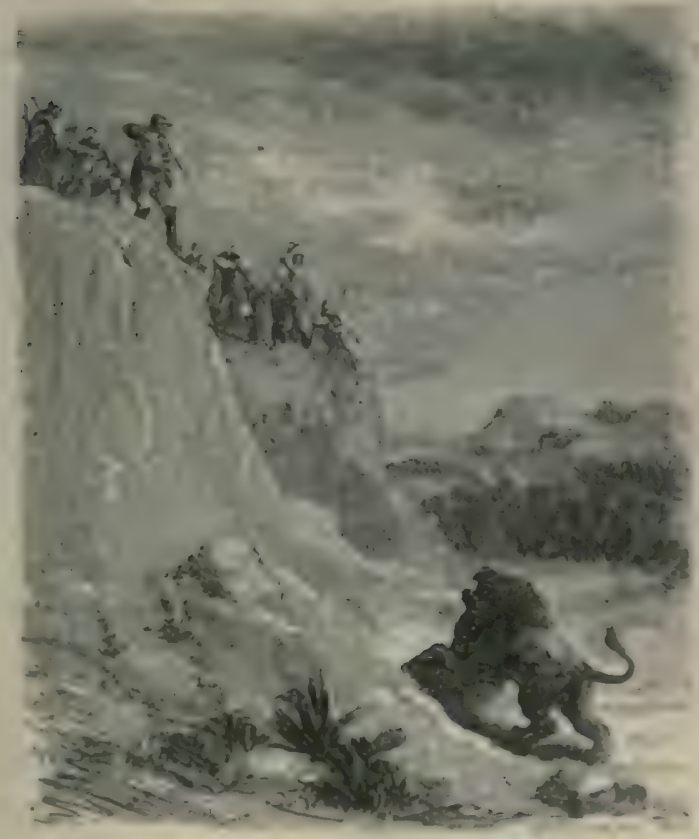

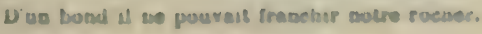

lben noms prit, et nous ne fumes pus longtempe a l.

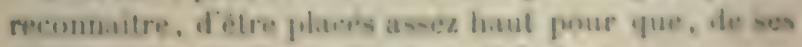
premiers bunds, al the put nous alteindre.

Il mous nurait certes fait un mauvais parli, malgr.

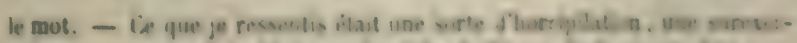

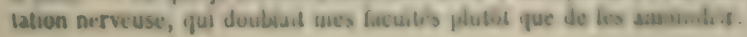


quelques halles qu'il recut d'une décharge presque générale, mais qui n'eurent d'autre eflet que de le rendre plus furieux!...

La hauteur de notre rocher était trop grande pour quiil parvint à la franchir; il le tenta néanmoins it plusieurs reprises par des sauts prodigieux, en poussant des rugissements qui agacaient nos nerfs et vibraient fortement en nous ( ${ }^{1}$ ).

J'avais réservé mon feu ainsi que El-MInkhtar, ce qui nous permit, après les efforls que le fier animal fit pour arriver jusqu'à nous, de le bien viser et de le tirer au bas de l'escarpement, daus un moment où il sapprètait à un nourel assatut.

La balle d'El-.Mokhtar lui entra par le poitrail, longea les côtes sous l'épaule droite et sortit par le flanc, ne lui faisant ainsi qu'un séton. La mienne, qui avait éti: tirée au front, n'eut pas cette destination, par suit: d'un de ses brusques mouvements de tête; elle pénétra dans la gueule, cassa une grosse dent du bas et sortit par la joue en entamant la mûchoire inférieure.

Ces deux nouvelles blessures portèrent au comble son exaspération; de sa queue qui sifflait dans l'air, il se battait les flanes arec rage, ses pattes de devant arrachaient des racines d'arbre et des pierres qu'elles faisaient voler en arrière comme lancées par une fronde.

Ce commencement d'action n'avait pas encore duré deux minutes, lorsque, voyant qu'il ne pourait nous joindre, le lion sembla prendre un parti et se mit it fuir vers notre droite; nous le pensions du moins, et jen fis la remarque a El-Mukhtar.

(') Les Aralws disent que le lion ruget dans le rentre de ceux qui l'attaquent. Cula nous semiba rrai, tant les viluations de celfe vois puissante vous pincitraient. 
Mais celni-ci, mieux anize, me ripendit: - D.trompe-troi, il ne fuit pass. il va nums tourner, ef hientit mus laurons sur nus derriares. - Je sous conserille at

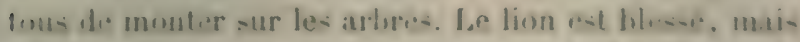
it $\mathrm{et}$ encore fort, et veut manger furly futin avant de mentrir::?....

Le conteril etait bon a suivre, nous fimes shois a l.s hite dm thenes los plus rappromluns pour y monter.

Vins fimes encore stimules dalls notre ascensiun

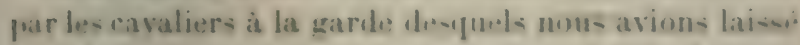
uns cheraux. Placés at cent rinjuate metres de nous -ur un pitun deses, ils virent le lion yui nous tournait: its mus crierent : - " 0 les gens! - vil. l... arbre. les arbres, - mili le limn! il vous arrive, - il vient -ur vous du coté droit... .

I:It.r.livenent, a peine atjons-nous jwhths sur nus rherses, dont la plupart, peol eleses, plovaient sous mutre poids, que nous le vime-s appuraitre, nous cherchant des yeux.

II etait effrayant d'aspect: sa gueule, à chaque conIraction, lancant une bcume sanclante, ses yeur injecto.s crmblaient jeter des leseurs rougres. Sa longue erinir.re nuire, herissepe raballue sur son fromt, le faisait pavalle enorme... Sa quese. frouellant autour de lui. atrallait les brandses des arleres.

Cielsit un des plos grands lions que Pon ait jamaiwis, et. dans lacton, il nous parut demesurememt long et haut.

II aurait pu nous cuwillir sur nos arhres comme des frommes moires, sil levit woulu.

Hien quion se dressaut sur sess pattes de derriêre. II poutait atteinitre le plus haut perehe dentre nous: mais le lien we grimpue pass commene la panther.. 
Il se contenta de courir d'un arbre à l'autre dans la direclion des coups de fusil et des cris qui les acconpagnaient.

Nous avions fini par nous griser au bruit de la poudre et aux rugissements de notre brave adversaire; c'était à qui l'interpellerait le plus fort, et Dieu sait comment, surfout quand il allait vers un arbre qui recelait un des nutres; - les cris redoublaient alors, afin d'altirer ailleurs son attention. Nous faisions de meme quand il allait vers nos chevaux, qui se cabraient et hennissaient de frayeur.

Le combat dura ainsi penrlant un quart d'heure; nous, tirant sur le lion quand nous l'apercevinns à découvert, entre les arlires; lui, courant dens toutes les directions vers les appels et les conps de fusil, qui de moment en moment lui causaient de nouvelles blessures...

Enfin, sétant une fois plus rapproch" de moi en me prètant le flanc gauche, je lui tirai ma troisième ballı. qui l'atteignit au cœur. Il s'affaissa sur ce coup qui fut salué des plus bruyantes acclamations!!!...

Le croyant mort, nous descendìmes de nos arbres pour aller le contempler de près, sans attendre, commr nous le disait El-Mokhtar, que son sanır se füt refroidi.

A peine avions-nous fait quelques pas vers lui, que dans un suprème effort de sa violente agonie il se releva sur ses pattes et fit deux ou trois pas comme pour s'élancer sur nous.

Nos fusils étaient déchargés, une lutte corps à corps dans ce moment nous tentait moins que jamais... Instinctivement nous fìmes volte-face et courûmes vers nos arbres tutélaires... Ceux qui en étaient les plus 
rapirucheis sy elancirent avee la conviotion que be lion flait sur leurs pas.

Xinurelle emotion. comme bien on penste.

M.ti- estui qui re-sentit la plu- forte a cet instant hat mun adjuint, M. Seriziat.

11 purtait une grande ceinture de laine qui sietait A.rombe fundant sa course; au monent wi il se crampumait a Farbre, elle sacroutia is un lon ssou et le

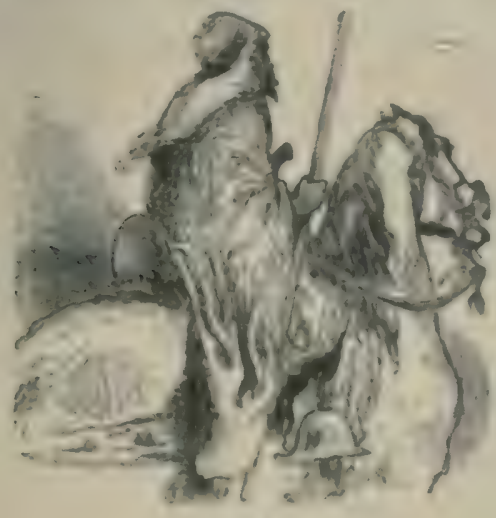

Ei - Mohblar

tira en arrice, il crut que ielait le liun yui lapprefuratait par son poleto: et aldait le metre en lam-

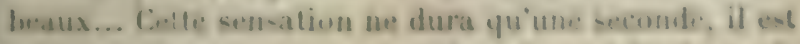

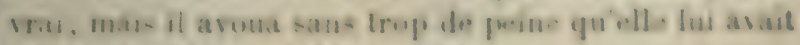
ete parfl-oliereament desagreable.

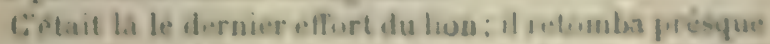
aussilit, en exhalant se w. dins un derner et courd rugissement.

Aims phones alurs fapprocher a motre aise.

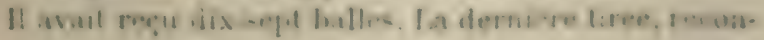


naissable par sa grosseur, avait causé sa mort en fracassant la cinquième côle et en venant se loger au coeur.

Nous fûmes longtemps ì repaitre nos yeux du spectacle de ce magnifique animal étendu à nos pieds; nous élions pour ainsi dire fascinés...

Les Arabes des environs, attirés par le bruit de la lutte, vinrent aussi contempler l'ennemi de leurs troupeaux. Hommes, femmes, enfants, rangés autour de Iui, parlaient et gesticulaient avec véhémence.

Ceux du douar, aux dépens desquels il s'était nourri quelques jours, ne lui ménagirent pas les reproches.

“ Dieu a enfin pris justice sur toi, lui dirent-ils. C'est la vengeance des hrebis que tu as mangées, qui pèse sur ta destinée! - Ton jour est arrivé et c'est ce jour qui t'acquitte de la dette de sang! ”

Des jeunes gens, moins mesurés dans leur rancune. lui disaient: "Eh! fils de chien, tu as trouvé d'autres. adversaires que des boufs et des moutons! - La poudre '’a mangé à son tour, les balles t'ont cassé les os!"

Les femmes, plus surexcitées que les hommes peutitre, lui jetirent aussi leurs bravades à la face. - " $U$ brandon de feu, voleur de nuit, mangeur du bien des pauvres! tu ne rugiras plus pris de nos tentes! Tu ne feras plus peur à nos enfants!!!" "

Et tous, en s'extasiant, répétaient: "Quelle tête, ủ Dieu, mon maitre! Quelles pattes puissantes! Quelles griffes pour déchirer la chair! Quelles dents pour moudret les os!

" - Oui, c'est bien là le roi des animaux ...."

Retiré à l'écart, j'étais, moi, pendant cette scène, sous l'empire d'impressions différentes; l'excitation d"u combat était tombée, je consiclérais ce lion qui ne m’a- 


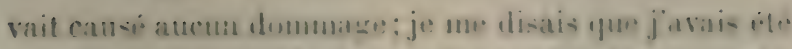

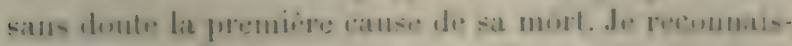
sais nasoir elé mi que par la vanile de punare dire : " dai classe le lim.n.

lit je Iromvais que 10 iat un senliment me-suin it

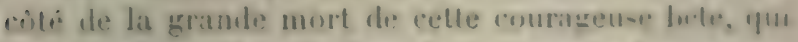

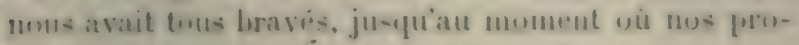
jertiles sirils avaient eu raisun de sas vie.

Lorsque je revins pris du liun, joutr lo litire charaer sur deux chevaux atlelés le front. jeus comme un remords, et assurément jelats tri-to.

Peu apres, je remarpluai fue cen sentiment dat fartacé par la plupart des areleurs de re dramr.

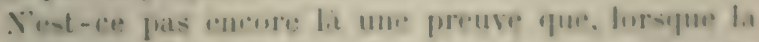

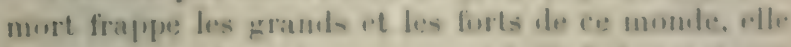

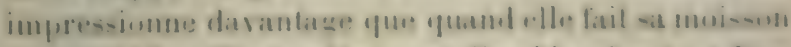

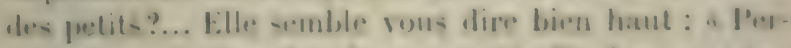
somme ne trouse grace devant moi! a lit alors on emm.

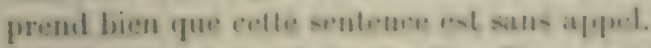

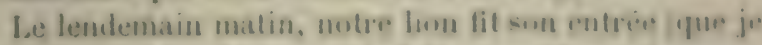

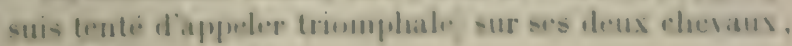
dans Ténict-el-Iliul.

Cis serul uaurait fun le purler:

Tous les sobledals de la garnion, la pongulation du vil-

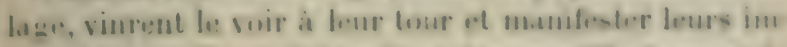

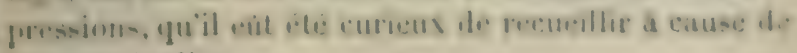
Iour originalité.

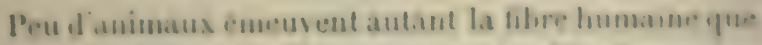

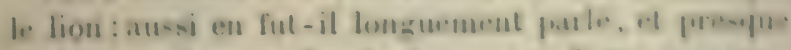

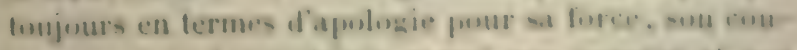
rage, verlus si fuppulaties parmi mos pous alls of thes 
32

LES CIIASSES DE L'ALGERIE.

solduls, qu'ils aiment à les louer partout où ils les rencontrent.

Ce n'est que bien avant dans l'après-midi, lorsyue la curiosité fut satisfaite, que je jus le faire dépouiller de sa grande peau, que j’ai conservée en suuvenir de mon début.

A partir de ce jour, je ne rèvai plus que chasse au lion, malgré mes dernières réflexions et ma considriralion particuliere pour ce digne animal.

Ce premier succès m'arait mis au cour une véritable fassion que bien des fatigues, bien des courses inutiles et de longues nuits blanches passées a liaffît, n’unt pu affaiblir.

Celte premiere chasse me confirma en outre clans la risolution de ne chasser le lion désormais que seul ou avec un solide compagnon.

J'ai expérimenté dans mes chasses suirantes yue cela ralait mieux ainsi; les chances de succès sont plus farorables. Il y a moins d'accidents à redouter de la part des tireurs peu expérimentés, et enfin la gloriole, inisque gloriole il y a, - est plus avouable quand on frarrient à tuer son lion en tète-a-tète. 


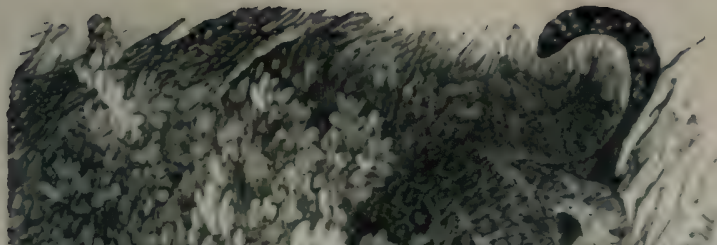
20.

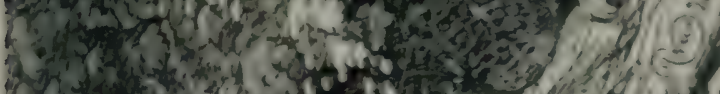

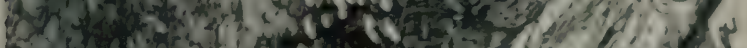

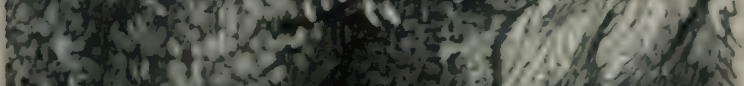

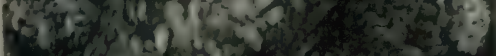

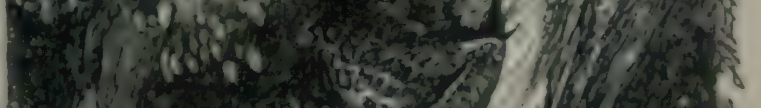
- 1 (1)

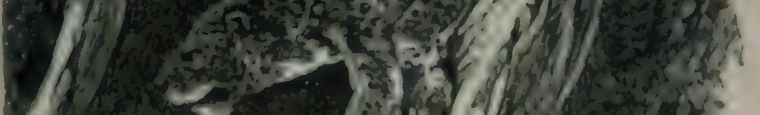

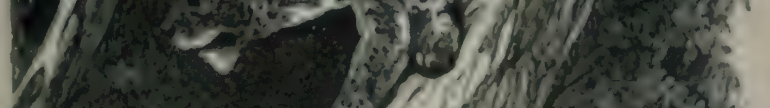

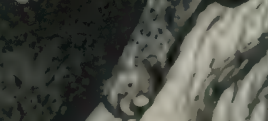

$\left(\begin{array}{l}1 \\ 2\end{array}\right.$

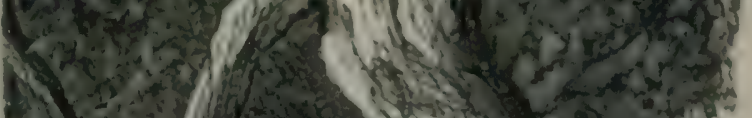

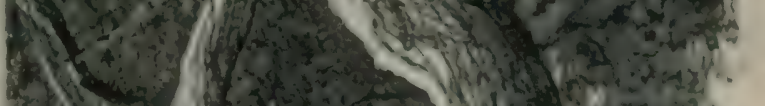

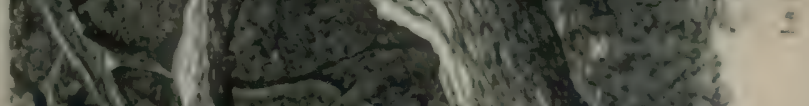
M.

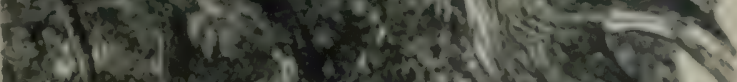

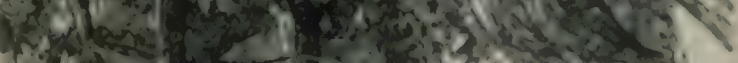

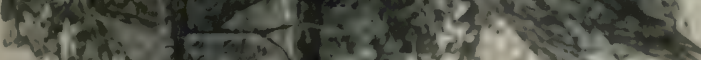

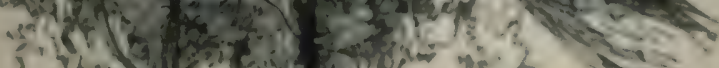

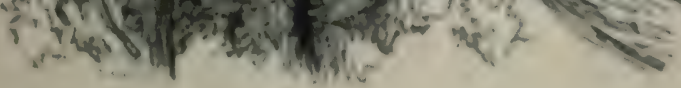





\section{IA PREMIÉRE PANTHÉRE}

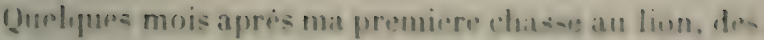
Iralues vintent me prévenir yue dos vaches et des girni-ors, appartenant a un douar des Muland-sili-Yahia ,

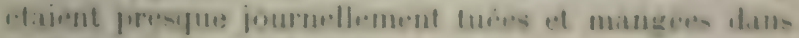

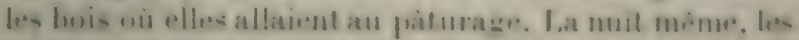

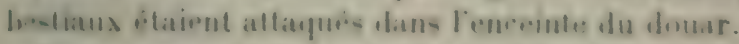

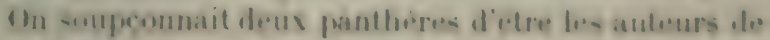
a... degals: les pauvres gens ansyuels apparlenatent les hedes mangere etaient dans la consternation.

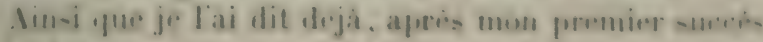

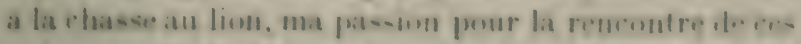
anmux setait deme-urement accrue: je ne sonzes.

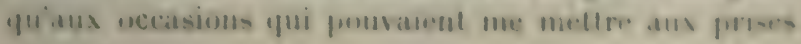
asee ens.

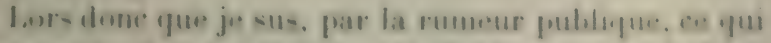

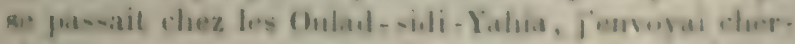
rher le chef du demar pour Ini demander des detaik

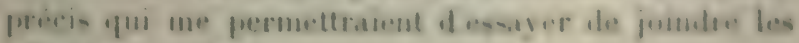

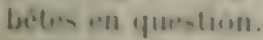

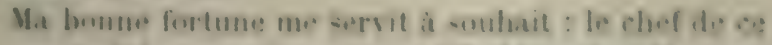

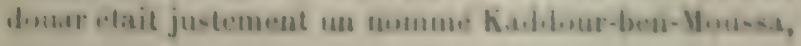


qui, l'année d'avant, avait eu une lutte terrible avec une panthère et conservait rancune à la race féline, on va voir pourquoi.

Le campement d'hiver ${ }^{1}$ ) de Kaddour-ben-Moussa et des siens était situé dans la partie la plus accidentée et la plus boisée du principal contre-fort de la magnifique forêt de cèdres de Téniet-el-Hàd, dans un lieı nommé la caverne du Fantôme.

Presque tous les ans, ils avaient dans cet endroit, privilégić, du reste, comme site pittoresque et grandiose, des bestiaux dévorés. J'année précédente, notamment, Kaddour en avait eu plusieurs de mangés. Un jour surtout, un jeune taureau auquel il tenait beaucoup avait été tué par la panthère en plein midi.

Kaddour, exaspéré de loutes ces pertes, se décida enfin à aller attaquer à son tour la panthère dans son refuge et à lui faire expier toutes ses déprédations.

Pour mener à bien cette résolution, il fit un appel à la bonne volonté de ses frères et de quelques amis du voisinage, qui s'empressèrent d'y répondre.

Ceux-ci, au nombre de huit, après avoir bien chargé leurs fusils et leurs pistolets, s'etre promis aide et sccours réciproques, se mirent, arec Kaddour à leur tìte, sur les traces de la panthère.

Ils les suivirent pendant assez de temps sur une longue crète boisée et rocheuse, qui aboutissait à un escar-

(') Les Arabes du Tell, quoiqu'ils ne soient pas nomades, cliangent plusieurs fois de campement dans le cours de l'année, surtout au printenips et à l'automne, pour mieux faire paitre leurs bestiaux et fumer les terres par le parcage. Le campenent d'étí et celui d'liver sont les plus stables, le premier à cause des récoltes à faire, et le second en raison des labours et d'une installation plus confortable en gourbis et abris pour les bestiaux, quie nécessite la saison froide. Le campement d'hiver dure de quatre à cinq mors sur le inême emplacement. 


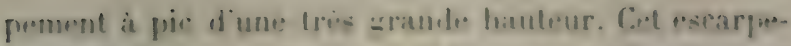

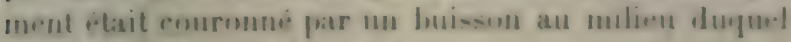
gelevitit un beatu cheme rert.

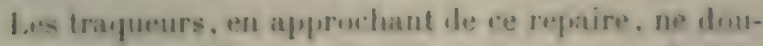
taient pas que la beto yü ils suivaind ny liat refugio...

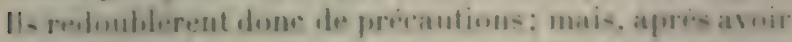
fonille be lonisson, ils recommorent quil elitit vide. Ils

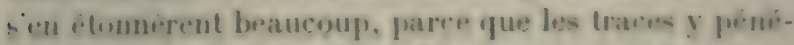
trairnl et ne reparaissaient plus dans ancune direction.

II- allibent revenir sur lecur- pas, lorsque hididour savi-a de lever la tóte el do regarder daus larbore.

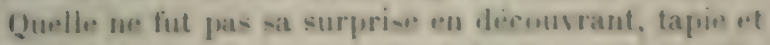

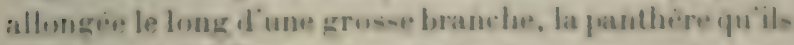
fusursuivajent: Pour se soustraire at une rencontre que ans iloute olle redoutait. elle as at srimpe enmme un

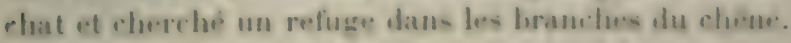

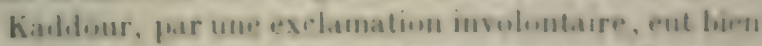
bite altare latlentitu de ses compazmens sur lour enuemie, of relle de celle-ci sur lur-meme en la fisant du regard el en laju-tant de -un fusl. Uuatre un cinq eoups

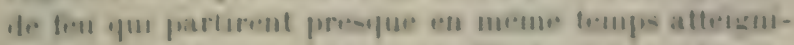

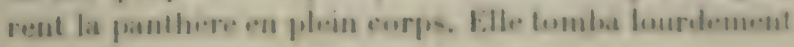
au pied de liarbre.

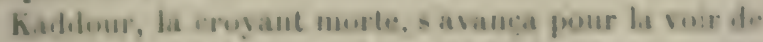
fres, mals mal lu en prit. Ia paullure avalt encole une

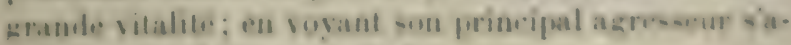
1.untre, flle sielanea lers lut.

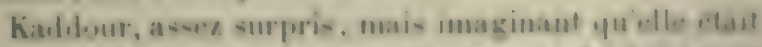

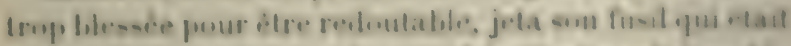

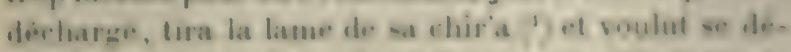

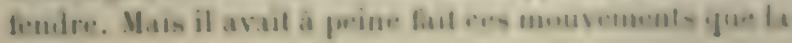

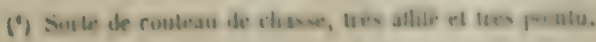


panthère était sur lui, la gueule béante et la griffe haute!...

D'un mouvement instinctif et comme pour se préserver des morsures, Kaddour enfonça résolument son bras gauche dans cette énorme gueule en essayant d'en saisir la langue; en mème temps, de sa main droite, il portait des coups de son arme sur les pattes et sur le flanc de la panthère.

C'était, comme on le voit, une véritable lutte corps à corps, dans laquelle n'osaient mème pas intervenir les frères du malheureux, qui appelait à l'aide.

En quelques secondes, il fut renversé par la panthère, qui s'accroupit sur lui et se mit à le labourer de ses griffes et de ses crocs.

$A$ cet instant seulement, ceux qui n'araient pas tiré saisirent un peu d'immobilité dans le groupe, appuyèrent le bout de leurs fusils sur les reins de la bête, firent feu et la foudroyèrent sur le corps de leur infortuné compagnon.

Kaddour ne donnait plus signe de vie; il avait le bras gauche broyé jusqu'au coude, la peau du crâne et de la figure était lacérée, l'œil gauche arraché; sa. poitrine, enfin, sur laquelle s'étaient exercées les pattes de la panthère, n'était qu'une plaie.

C'est dans cet état que Kaddour fut rapporté dans sa lente, oì l'attendaient les lamentations de ses femmes et de ses enfants.

Cependant on s'aperçut qu'il respirait encore. On lui prodigua tous les soins iniaginables, et, contre toute espérance, il guérit de ses graves et nombreuses blessures. Mais le pauvre homme resta défiguré, et, pour ne pas être un objet d'horreur, il était obligé de se voiler la moitié de la face avec son haïck. 


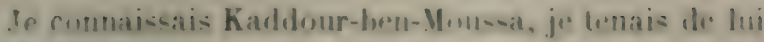
t de -..- rompagnons les details qui prevident.

- Eh trean. las dis-je. quand il vint me toucher la main. les pantheres ne veulent done te lassere aucun repit? list-il vrai quelles sallaguent eneore a tes lexliatus?

- C̈est vrai, me répundit-il, ceat la volonti de Dieu: qur puis-je y laire, du re-te? tu wis commont fai ete traile. o Et il me montrait son leras nutile. sat lisure qui ne conservait plus rien d humain. - a Je ne fuis mine plus pscilyer do me venger $"$, ajouta- 1 - il alec tristese ef risignation.

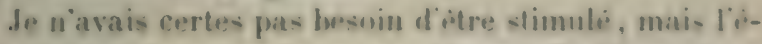
melion que je ressentis en con-inlerant Kadhlour - leenVonsas anementat encore mose de- ir de lenir une panHhere an bout de mon fusil. Je lui dis ators mon projed,

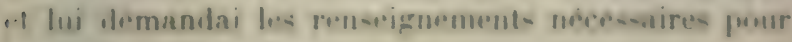
lo meltro it excecution.

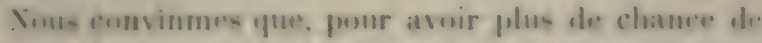
liver la lo.el. yni venall chaque soir sallayuer a -...

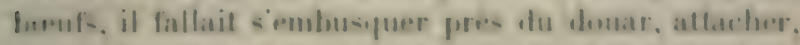

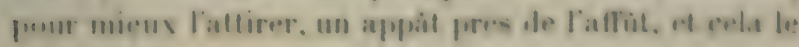
- air même.

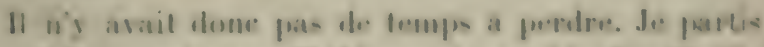

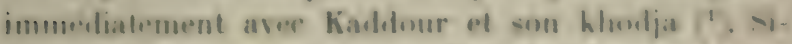

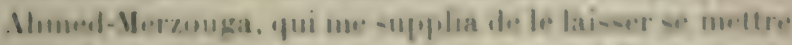

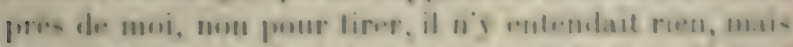

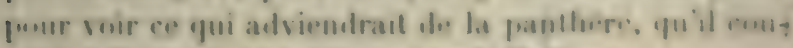

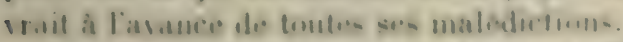

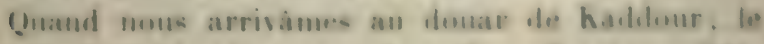

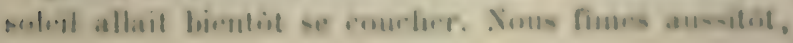

(i) Alowlje, acritaire. 
avec l'aide des gens de bonne volonté, un trou dans lequel je devais me placer arec si-.lhmed, qui y tenait plus que jamais, depuis qu'il avait entendu les récits lamentables des femmes et des vieillards.

Le trou creusé, je le fis entourer de petites branches d'arbre de facon à simuler un buisson naturel; puis je fis attacher, à la distance de cinq pas, sur une petite élévation $\left({ }^{1}\right)$, les restes d'une génisse tuée la veille par la panthère, presque à l'endroit mème où nous étions. Cés dispositions à peine acherées, le soleil suétait couché; les bestiaux avaient été rentrés dans l'enceinte du douar, le moment était venu de nous glisser dans notre trou; ce que nous fìmes.

Les habitants du douar nous souhaitèrent l'aide de. Dieu, puis rentrèrent dans leurs tentes, en fermant. avec de grosses branches le passage de la haute haie qui les entourait.

Une fois dans notre affùt, nous primes chacun lis position qui nous parut la plus commode pour passer la nuit.

Je me préoccupai surtout de bien décourrir l'endroit où viendrait se placer la panthère pour achever les restes de la génisse; je dirigeai le canon de mon fusil dins cette direction. Cela fait, je me mis au repos et l'attente commença!

On a déjà écrit les Réflexions d'un chasseur à l'afpüt.

(') Sur une petite élévation, c'est-à-dire de façon à ce que la bête qui viendrait pour manger se détachàt bien nettement dans le ciel. J'ai tnujours eu soin d'établir ainsi mes affüts, ce qui m’a permis, même par les nuits les plus obscures, de bien voir les hêtes jui venaient röder autour de liappàt ou y mordre, et de placer ma balle a peu près oil je voulais; tous las autres procédés, tels fue mettre dı plosplıne ou un brillant au guidon, etc., ne valent pas la manitre que j"indinge. 


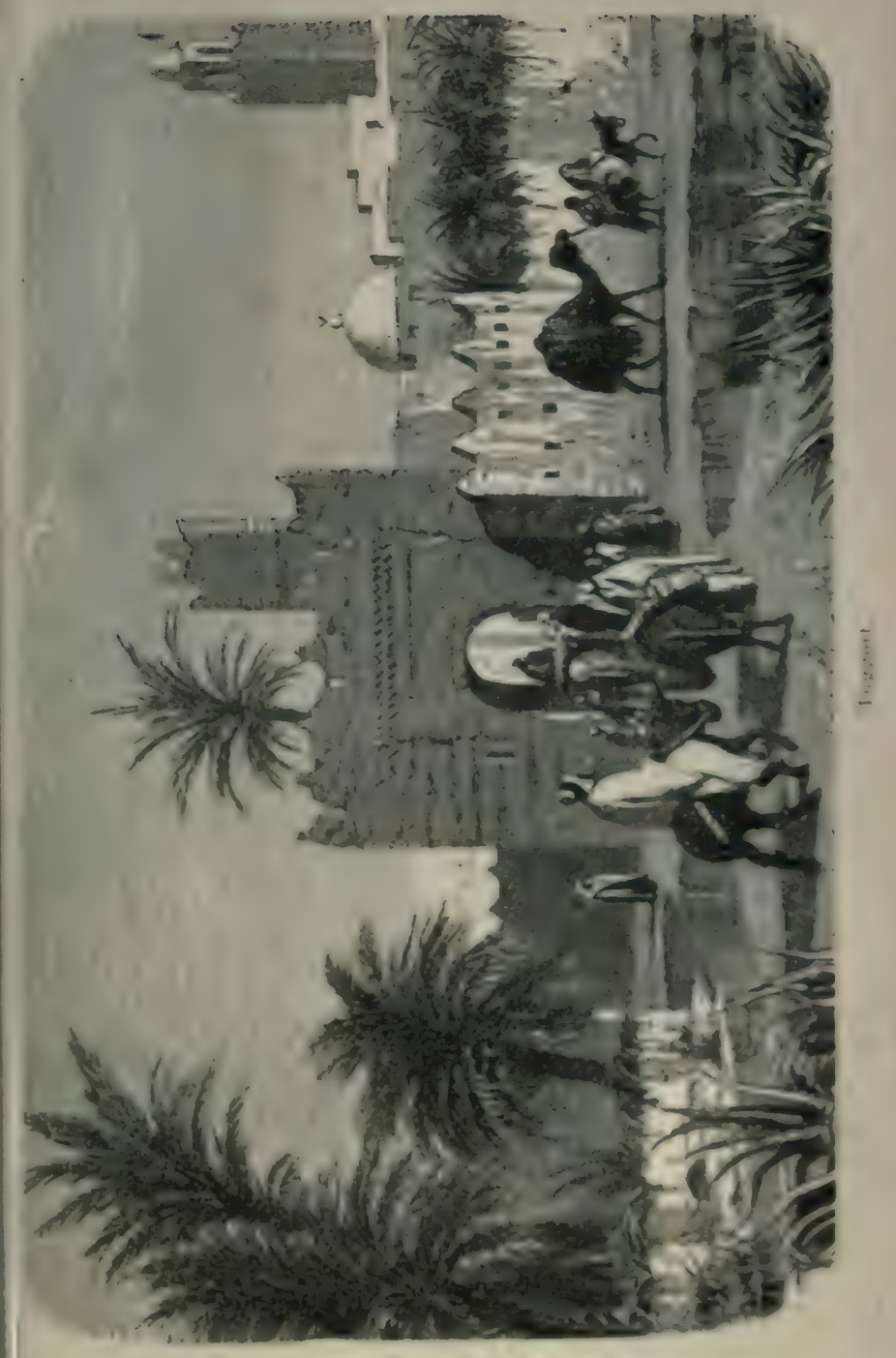



plusieurs vaches mangies en peu de jours! - Il doit même y avoir des petits? Certainement il y en a. Voila qui explique la grande consommation.

- Alors, une panthere au moins viendra achever le repas commencé la veille, ou il ne faudrait pas avoir la moindre chance. - Bon! cest très rassurant cela!

Troisieme consideration : - Mais si elle vient, ne flairera-t-elle pas l'embunhe? Ce buisson n'était pas là

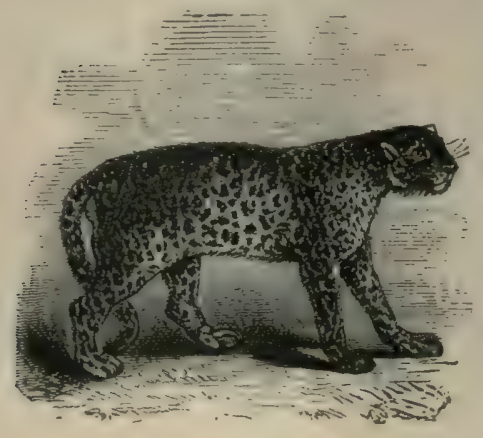

La pantière. jier...et il est assez jeu réussi. - Cette odeur de terre fraîchement remuée... roilà bien de quoi lui donner de légilimes soupcons. Mais quelle apparence?... - Il n'est pas probable que les panthères aient des notions exactes sur le temps que mettent les arbustes à pousser. Et puis il est bien connu qu'elles n'ont pas une très grande finesse d'odorat! T'rès bien encore.

Quatrième considération : - Comment se présentera Ja pranthère quand elle viendra mordre à la génisse, - de tlanc ou de face?

- Il vaudrait mieux de flanc, la surface est plus grande et le cour plus à decouvert. - Comment est chargé le fusil? Bien, j’imagine; bonne mesure de foudre, deux balles maricins dans chaque canon, capsules à bombes mises sur les cheminées pleines de poudre. Pas de ratés possibles ! - Très bien cela... 
- Que fera la pantheres une foris tirrese?

- Il eat rare que cot animal suit fue radide: ollo fomrera probablement sur le coup, à moius yue la luener de lat dotunation ne l'effrave ot la jefte de ciole. - (1) verra bien!

loi la folle du logis, qui a fait heanconp de chemin -ur ce- themes qui ne sont indiques que trés summairemsent, donne une attention toute patrticuliere a des

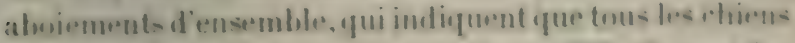

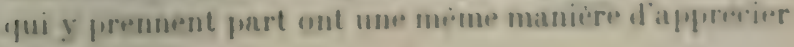
ce qu'ils fairent.

- E-t-e la panthere?... Ce doit etre elle!...

(1): alors... le corur - ien mite, il hat plus furt. Iat requiration deviont brugante. II faut ourrir la lumeles pour lui donner plus de jeu ef ne faire aucun lorut rivelatentr.

Dend minules. trois mimuless se passent. Ios elivens

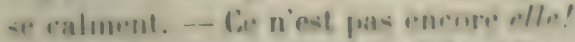

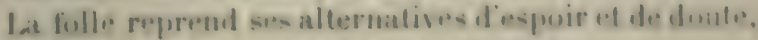
aves: variantes et eon-iderations nouvelles.

Il doit idge unse herures. Il gele... Jai tries froid ans

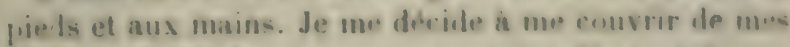

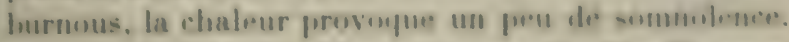
Dans la erainte de cealer au sommeil, jeseille mem compagnon qui dort dequis pres de dous labures. Je lui recommande de veiller ì son tour el de use presenir sil entenil quelypue cluse...

Ia procaution thait bonne. Je mienders. frendant

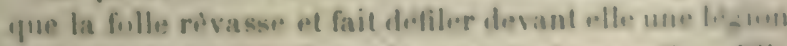

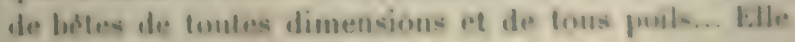
se emmplail en relle emmpaznie.

Dieu, yui en voila de grandes ! Connume elles paraissent féruces! Elles passent a regret en jetant de ce ciit. 
ın regard qui entre dans les chairs comme des grifes.

- En voici une horrible : elle a plusieurs têtes. G'est sans doute la bète de l'Apocalypse. Elle marche lentement. Mais comme elle grandit à mesure quelle approche! A qui en veut-elle avec ses gueules ouvertes?...

th ! il était temps ! Je me reveille par la sensation d'un coup de coude dans les côtes. Je reprends possession de moi en rappelant la folle du logis à la réalité et à l'appréciation exacte de la situation.

"-Qu'est-ce? dis-je à voix basse.

" - La panthère est venue, me répond de même SiAhmed-Merzouga; elle s'est montrée au-dessus de l'appàt, et s'est enfuie aussitot. "

Je suis confus et peiné... Quelle occasion je viens de manquer!... Peut-être ne reviendra-t-elle pas? Aussi cst-ce assez absurde de s'endormir quand on vient pour guetter toute une nuit!...

Tout en faisant ces réflexions, je reprenais ma puic de la première heure.

Les chiens s'étant mis de nouveau à donner très fort, je me remis à espérer.

La panthère rôdait évidemment autour du douar, a en juger par les différentes directions que prenaient les aboiements.

Au bout d'un quart d'heure qui me parut bien long. ju vis poindre, derrière le petit tertre où était l'appàt, la tête d'abord, puis la moitié du corps de la janthère.

Je voulus aussitôt l'ajuster, mais mon fusil, amené - $\quad$ un peu trop brusquement à l'épaule, résonna contre ıne branche. Ce bruit, quoique faible, fut entendu de la panthère, qui disparut de nouveau.

Malédiction! je n'en viendrai pas à bout. Quelle dé- 
Jorsque la panthère passa sur nous en nous courrant des débris du buisson, je ne cours, je crois, aucun risque d'itre démenti, jas plus qu'en ajoulant que notre satisfaction fut grande d'en ètre quiltes à si bon marché.

J'étais sûr d’avoir bien touché ma panthère; si elle nétait morte elle ne devait pas en valoir mieux, et j'esfrírais la retrouver assez pris du douar le lendemain matin.

Tout en me débarrassant des branches que la panthre avait foulées sur nous, je me demandais si en $\therefore$ 'élancant vers l'endroit d'où venait le coup qui l'avait frappée ell: lavait fait avec intention, ou si la pente naturelle du terrain, blessée comme elle devait liètre, ne l'avait pas simplement entrainée dans sa chute.

Je penchais pour cette hypothese; mais Si-AhmedNerzouga, clerc s'il en fut, possédant ses auteurs sur le bout du doigt, prétendit qu elle l'avait fait avec intention, que cela sétait toujours ru ainsi, que El-Donmiri $\left.{ }^{1}\right)$ lui-même avait écrit de la panthère qu'clle ćtait très vindicative et faisait un mauvais parti à ses agresseurs. Bref, le cher homme n était pas fâché de se figurer qu'il avait couru quelque danger en ma compagnie, et comme, en résumé, il l'arait fait de gaieté de cœur, je ne voulus pas avoir l'air de douter de son assertion, et lui enlever le relief que cela lui donnait dans sa propre appréciation.

Nous agitâmes ensuite la question sur la meilleure manière d'achever notre muit. Il nous parut qu'il n'y en avait pas d'autre que de rester dans l'espece de fosse où nous étions, en nous couvrant le mieux possible de nos burnous.

(2) Savant auteur arabe qui a écrit sur l'histoire naturelie. 


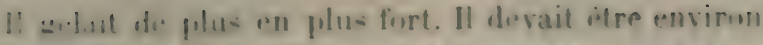
ume honre apros mimuit; uoms avions encore quatre on cind lowares de belle étrile. que nous domes passer ain-i, partie en sommeillant, partie en grelottant.

I la printe du junr, los gens du donar, trés impantimbl-de connailre le rémultat de mon comp de fu-il, -orturent aser leurs rhiens ef se dirigerent vers un hasfind qui a. trouvail it 80 metres derriere et au has de

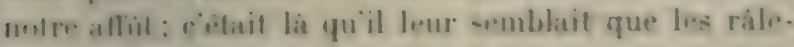
un.ut- de la panthere avaienl fini.

1,..5 rhiens, excites ot lances en avant, arriverent bientiot press de lat hete, qui itait raiste morte... II.

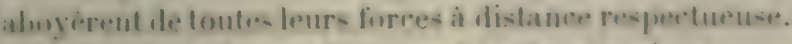
C. hruit nous deguredit, mon khodja of moi et nonmit sur pied.

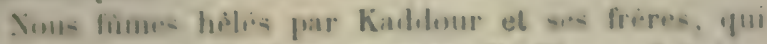
ums crierent : a Le tigre est mort (1)! Dind a fait fus-

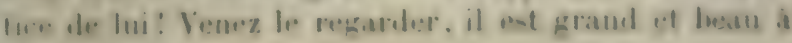
1.ult : .

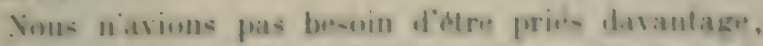
mons areourions de coule la vilesse de wos jambes raidies par le froid.

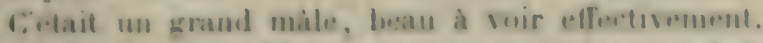
Ves deus bal!es. quil avait recurs en pleine putrone.

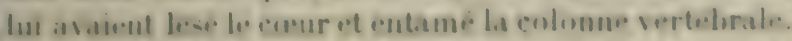
II Iu avait fallu sa puissante vitalité pour quu il ne re-tit fus sur le coup.

Ciny luommes le prirent par loes pattess et la queue. A l. monfirent an donar, wi fiemmes. enfants et siont-

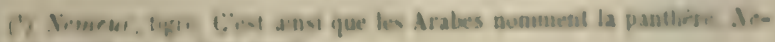

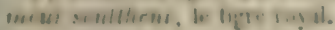


lards, vinrent l'entourer et le maudire jusque dans sa postérité la plus reculée.

Après nous être réchauffés a un feu bien flambant nous repartimes pour Téniet-el-Ilàd. Deux heures apris, la panthere y arrivait aussi, portée sur un mulet.

Nous fùmes complimentés, cela va sans dire; surtıut $\mathrm{Si}$-Ahmed-Merzouga, qui s'était constitué l'historiographe de l'aventure. Il en eut pour une semaine a l'écrire et à la narrer à qui voulait l'entendre.

Mon excellent khodja ne manquait jamais d'appuyer sur le bond de la panthère et de faire remarquer que cétait par gràce divine toute spéciale que nous avions dù de nètre pas mis en pièces. Aussi chacun lui disait, comme je lo dis moi-mème en terminant ce récit: "Dieu soit loué! "

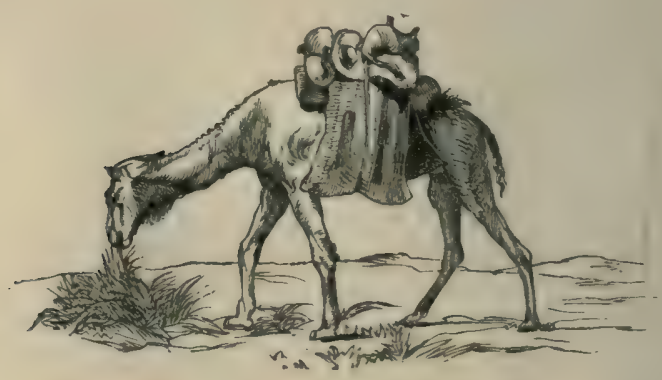




\title{
L.t \\ CHASSE A L'AUTRUOIE
}

\author{
DANS LE SAIIIA ALGERIES
}

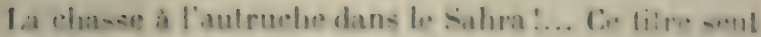

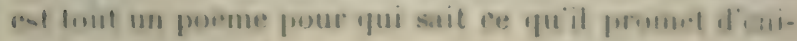
vrantes émotions!

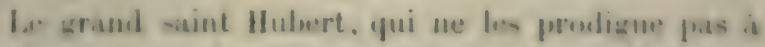

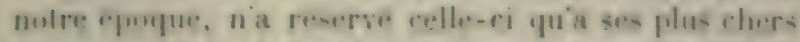
elus : ans-i lui suis-je a jamais reconnatssant de leferetion partieuliere quil a faite de quelyues-ans de imes amis of de moi pour leur reveler celte chasese, - $-i$ ed. trainante ef si rare - que bien peu d'turoperens peuvent se vanter de l'asuir faile.

lin jorence de la narration que je vais entroprowir. jeprouse serfieusement le regret de n'asoir ui le stgl. ni la couleur equil fandrait pour bien rembere ce que jai vu et eprouve a cotte chasse.

Cat husulale aveu ćtant fait en maniere de conjuratios el par anequit de conseciener. jaburile mon sujel.

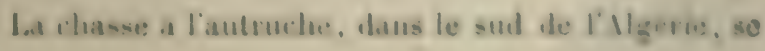


fait principalement sur le grand plateau situé entre Laghouat au nord, les Beni-Mzab au sud, le pays des Dulad-sidi-Chikh à l'ouest et Dzioua à l'est.

Ce plateau, d'une superficie de deux mille lieues carríes, est, pendant l'hiver, une partie du printemps et de l'automne, le pays de parcours des grandes tribus sahariennes, les Larba, Oulad-Nayls et Zouas, qui y font pacager leurs nombreux trompeaux de brebis et de chameaux.

Pour les nomades et les vrais chasseurs, ce désert possède un charme infini, de puissantes attractions, qui semblent émaner des premiers âges. avec le ressouvenir de la vie pastorale et contemplative.

L'existence biblique des patriarches se retrouve li tout entière; en effet, abordez ce douar des OuladNayls, et vous y retrouverez Laban, Jacob, Rébecca, Joseph et ses frères avec leurs tentes et leurs troupeaux. - Causez avec eux, ils vous diront leurs pérégrinations pour aller au loin chercher le blé ou les gras pâturages.

Avez-vous soif? Voici une fille d'Ismaël aux grands yeux noirs, qui va rous désaltérer avec le lait des chamelles ou l'eau de goudron, à votre choix.

Avez-vous faim? Dites : "Je suis l'hote de Dieu!" Le chef de la tente s'avancera vers vous en disant: "Sois le bienvenu!" et un mouton sera immolé en votre honneur.

Mais ce n'est pas pour peindre ces scènes des temps primitifs, encore vraies aujourd'hui, que j'ai entrepris ce récit. - Le désert dont je parle n'est plus habité à l'heure où nous allons $y$ chasser l'autruche. J'en reprends la description. 


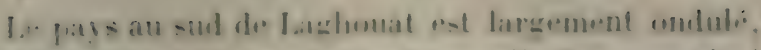

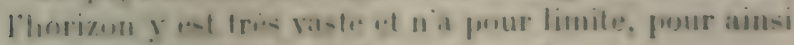
lime. pure la fatile.se de la vore humaine.

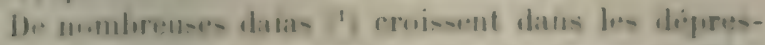

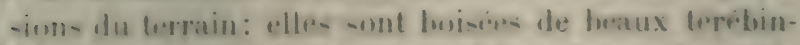

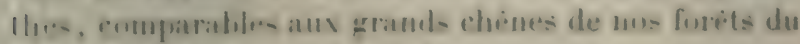
Anrel, et de bui-suns de jujuliers sanvaenes.

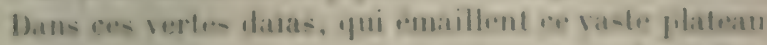
du sul, comme los nomres momeluelures lachent une

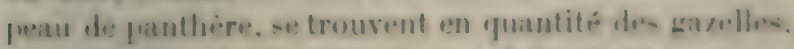
des mutarios, des lievres, des perdrix, dos gangas, et bien diutres foperes enoure, anssi rheres aux nituralietes quiaux rlassomer

L.er rele du sul est coumert d'arbustes et de plantes, t.e. 4 que :

I... S Salsulas ligueuses.

\section{L'If 'lianthime,}

LArmoise,

Io Rauthérium,

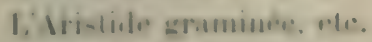

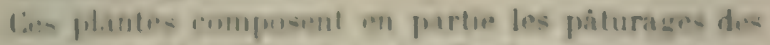
Irmupans et du gibier dus suel: olles leur donnent eetle.

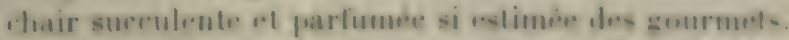

II u'y a ni sources ni cours d'eau dans ce lorritioire: los orages, los rares pluies aliver, alimentent sent-

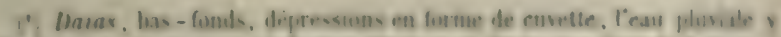

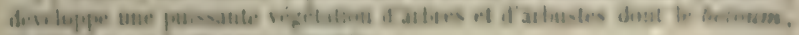

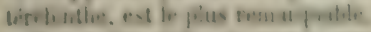

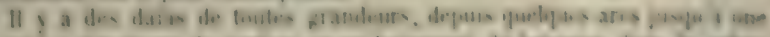

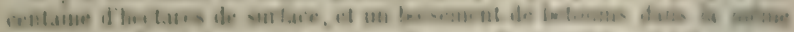

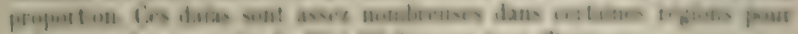

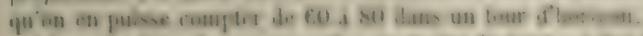

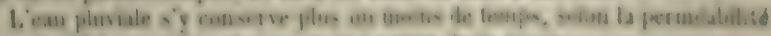

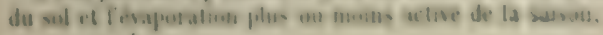


des réservoirs naturels qui se forment dans les daïas et le thalweg des vallées.

Ces rícrvoirs, appelés r'dirs (') par les indigines, en raison de leur peu de durée et des déceplions nombreuses qu'ils ont causées aux gens altérés, ne conservent leur eau que pendant quelques semaines dans la saison froide.

Ils la conservent bien moins longtemps cncore en élé; d'où la nécessité, pour les nomades, à l'époyue de la sécheresse, dabandonner malgré eux ces bienaimées terres de parcours, et de remonter vers le nord dans la région des eaux vives.

C'est alors que le plateau devient désert, comme je laai déjà dit, depuis le mois de mai jusqu’au mois d'octubre; il n'est plus traversé à cette époque que par de rares caravanes qui se rendent à Tuggurt et au Mzab.

C'est aussi dans cette saison que les autruches, chassées des régions plus méridionales par l'ardeur du soleil, envahissent cette parlie du Sahra pour y chercher lombre et la pâture.

La chasse à courre à l'autruche se fait donc dans les plus chaudes journées de l'année. Elle dure quarante ou cinquante jours, du 25 juin au 15 août. Les Arabes disent que c'est la chaleur plus encore que la vitesse des cheraux qui amène la capture de l'autruche.

Cet entraînant et rude exercice est le monopole de quelques tribus qui, avant que notre domination se fut établie dans le sud, y joignaient l'industrie moins licite du pillage des cararanes.

Ces tribus sont:

(') De r'deur, tralir. 
avons tous quelyues àmes sur la conscirnce... Dieu seul est parfait!... 》

Quelques àmes sur la conscience!... Hélas! uui; uи de leurs chefs, Toumi, dans ses moments d'expansion, en avouait une vingtaine: "Jai tué bien des gens combattant les caravanes, me disait-il un jour, beaucoup plus qu'il n'est permis à un bon croyant!... Après tout. chacun glane son existence comme il peut!... Nous n'avons fait que ce que nous ont montré nos pères! mais vingt hommes tués!... cela me préoccupe pour le' jour du jugement, - et souvent j'y songe !!! »

J'ai hâte de dire qu'en dehors des méfaits antéricur: dont leur conscience peut ètre chargée, méfaits yue l'on retrouve plus ou moins à l'état latent, chez les Arabes qui échappent à une autorité vigoureusr, lé Mrokhalifs sont hospitaliers, serviables et franes comme de vrais chasseurs.

Leur étude, pour quiconque connait leur langue el leurs usages, est pleine d'intérèt. Il va sans dire qu* l'on peut actuellement parcourir en toute sùreté arec eux le pays qui a servi de théâtre à leurs cxploits.

J'aurai mème à raconter plus loin l'extrème sollicitude qu'ils ont montrée envers mes compagnons d:chasse et moi.

Ce qui précide donne une légère teinte du paỵs et des gens qui aident à le parcourir; disons à présent un mot de l'outillage nécessaire pour passer un mois il li. chasse de l'autruche.

Le chameau est l'auxiliaire indispensable; sans cut excellent animal, qui pussède la faculté de rester en éti: sept ou huit jours sans boire, on ne pourrait vivre dan ce pays, si judicieusement nommé le payss de la soif. 


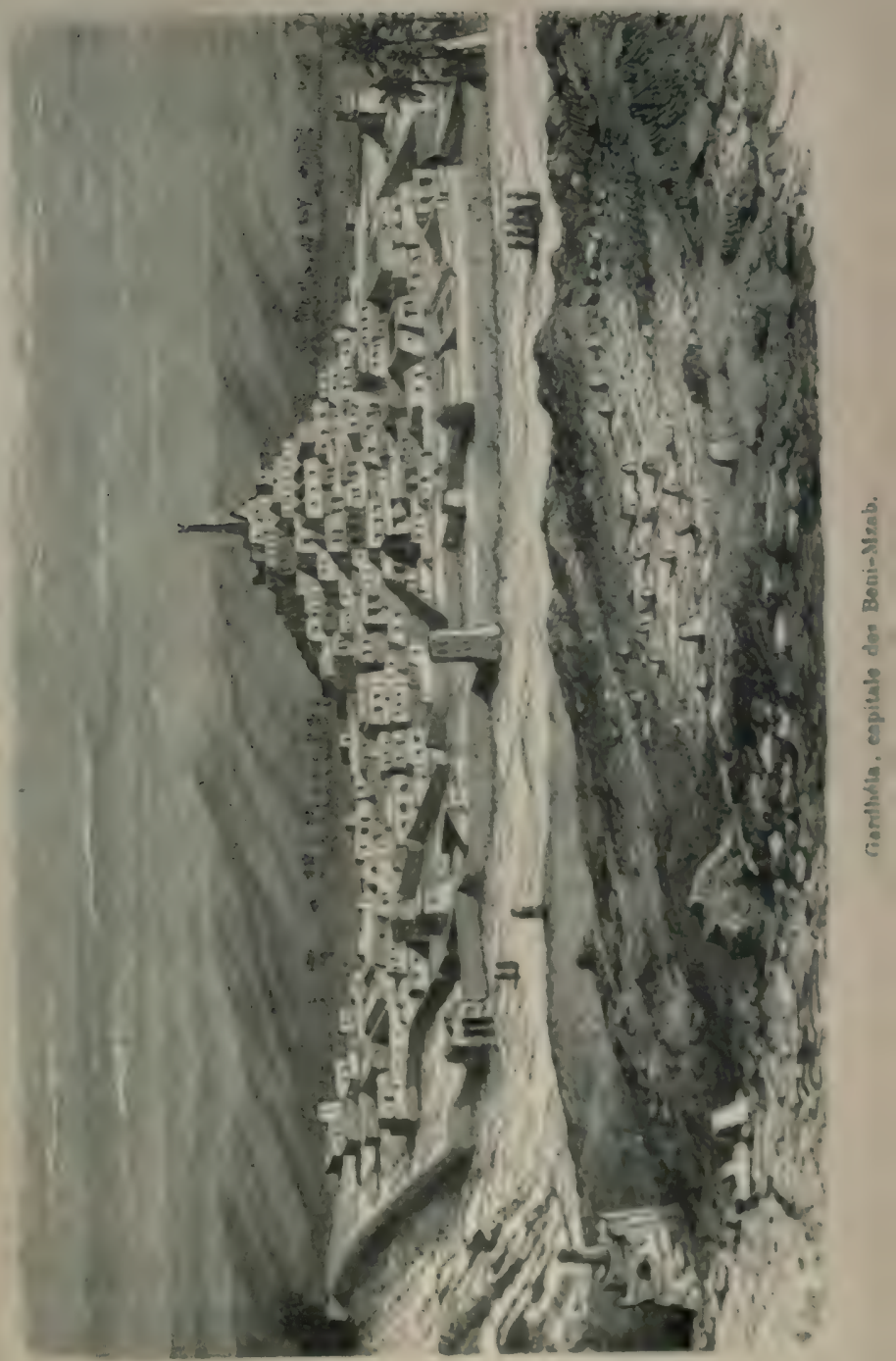





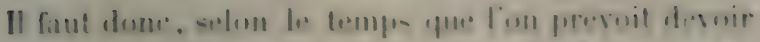

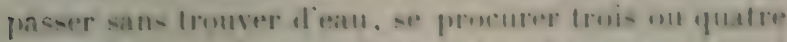

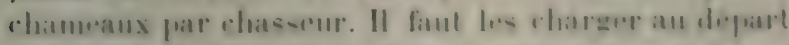

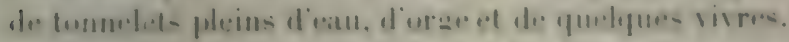

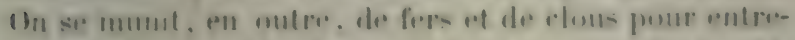
lenir la firrure de- chevaux, de sel punt saler les depouilles des autruches forcées.

\section{Inutile d'emporter} des tent s: le feuillage des betoums est un abri bien préférable a tout autre dans celte saison.

Chaque chasseur prend avec son fusil une quantité sutlisante de poudre, balles, plomb, pour assurer la nonrriture quotidienne.

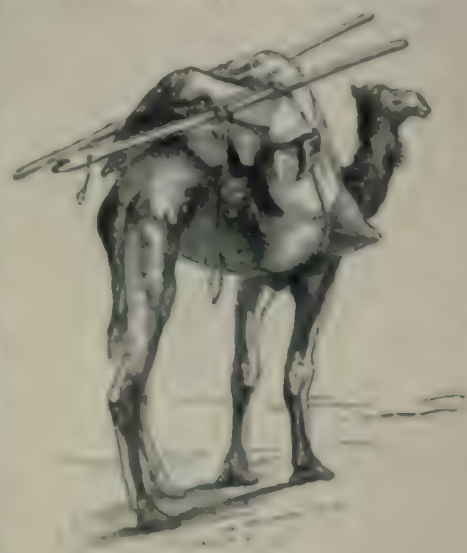

L. chinmean.

Les chevaux destinés a courir Tautru-he deisent stre entraines pendant fuune jours ou trois semaines.

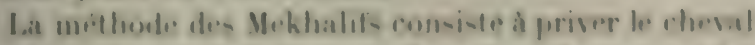

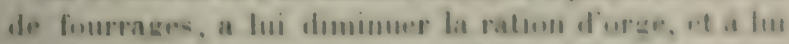

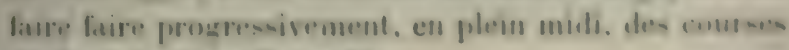
de deres a quatre leeves (1).

II nest pas indifferent dictre bien rensenge sur la

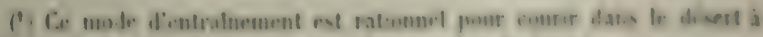

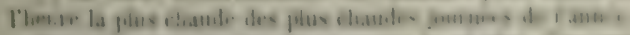

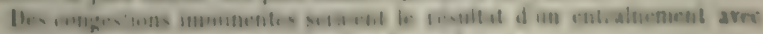

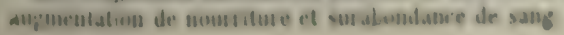


rigion qui recile le plus d'autruches, quand on vent commencer la chasse.

Aussi, quelques jours avant le départ, est-il opportun de faire explorer le pays par des éclaireurs expérimentés, montés sur des mahris ( $\left.{ }^{1}\right)$.

Cies éclaireurs recherchent en mème temps les r'dirs qui auraient conservé de l'eau.

Celle dernire découverte est tris importante, parce. yicelle permet de renoureler la provision sans obliger a parcourir de trop grandes distances, et parce que l'on est sûr de trouver dans le voisinage clu r'lir des autru-ches yui ont pris l'habitude de venir s'y désaltérer.

Je savais, en allant prendre le commandement du cercle de Laghonat. que j'aurais l'occasion de faire de: chasses tout à fait nouvelles, entre autres celle à l'autruche; et Dieu sait combien mon imagination, longlamps à l'avance, avait brodé et caressé cette perspective!

J'eus bientot fait la connaissance de deux caïds des Mekhalifs, Bel-Abbès et Toumi, personnages que je considérais beaucoup, tant à cause de leur cachet particulier d'originalité que de leur qualité de grands chasseurs d'autruches, et, il faut bien le dire, de mes projet. futurs.

Après aroir sourent causé arec eux et recueilli dc leurs récits tout ce qui pouvait m’intéresser, je leur avais fait connaitre mon désir de me joindre à eux pour les prochaines chasses à l'autruche.

Ils avaient gracieusement accueilli ma requète, à la suite de laquelle il avait été convenu que je serais dé-

(1) Mahris, chameaux de course qui possèdent une grande puissance de forcmotion. lis sont aux chameaux ordinaires ee qu'est le cleval de pur sang au cheval de trait. 
surmais des lears, initie a tous leurs proparalifs et infurme du jour vì eommencerait la chases.

Jisais done bien prepare physiquement et meralement. Lorsfue arrivat enfin ce jour lienheuresux !

Do.s la veille, javais été ralliv par M. Jo lieulonant Philebert, commandant le poste de Djelfa. qui matait demande it se juindre a mui : passionne cha-centr, il il.ait

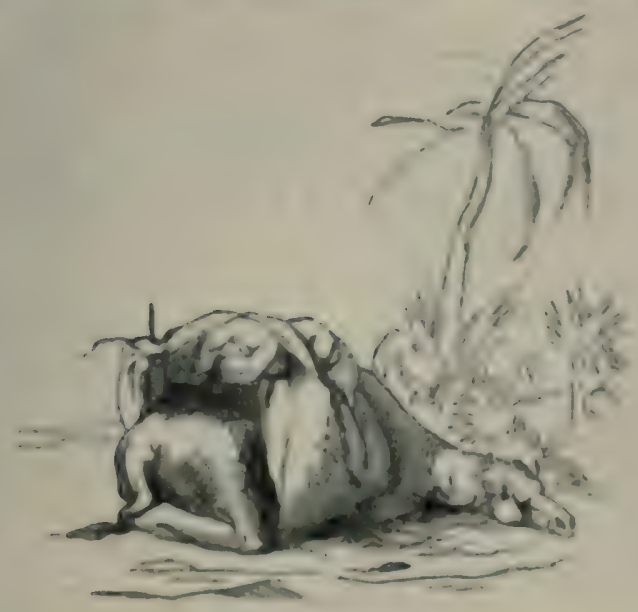

Lo repos du charreau.

anses tris impalient des se voir aux prian avee les anIruches.

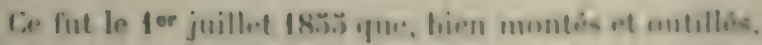
mutre caravane alumblamment pusuroue dienu, mons primes la direction du sud-est.

Javais domme render.-1mus hus Mehhalif a mi-eloemun

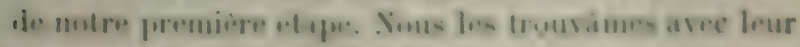
caratame aux datas de Bo.1- Mroug. 
Ils étaient trente chasseurs, y compris leurs deux chefs.

Nous avions de plus à notre suite quelques caraliers des Larba et des Oulad-Nayls, qui voulaient courir avee nous.

1 Le premier abord entre chasseurs est nécessairement consacré à l'intéressante question du gibier que l'on va poursuivre; on y mélange des vœux pour les succès futurs, des souvenirs des chasses précédentes, etc. Après donc que les Mekhalifs nous eurent salués et souhaité la bienvenue, j"échangeai quelques mots aver les éclaireurs :

- Eh bien, mes enfants, y a-t-il de la chasse, cette année? ( $\left.{ }^{1}\right)$

- Oui, il y en a le bien du bon Dieu.

- Prendrons-nous beaucoup dautruches?

- Invoquez Dieu et les jambes de ros chevaux.

-Où les avez-vous trouvées?

- Après avoir bien exploré, c est à Safel $\left({ }^{2}\right)$ que nous en avons ru beaucoup; elles y sont en troupeaux; le dernier orage les $\mathbf{y}$ a fait venir.

A cette alléchante nouvelle, les yeux de tous brillirent, un tressaillement de joie anima nos cœurs.

- Il y en a beaucoup de grandes, ajouta notre éclaireur en voyant le bon effet qu'il produisait; - je l'ai reconnu aux traces; - les mâles aussi sont en nombre, et, si Dieu veut, la chasse sera fortunée. Nous aurons grand plaisir et profit.

(1) Inutile de dire que je clserche, en traduisant nos conversations avec ies Mekhalifs, à conserver l'expression to la tournure des plirases empluyeiés.

(2) Grande dépression du plateau entre Laghouat et le Mzah.

Ce bas-font, a la moindre pluie, se courre de vigétation lerbacée, et, a cause de ceia, il est très affertionni des aulruches. 


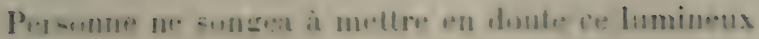
aligure.

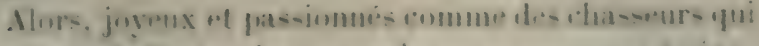

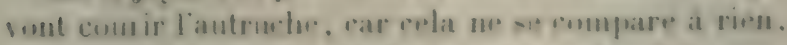

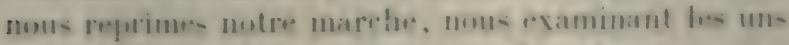
les autres, cherchant is revonnaitre lus mieux montos,

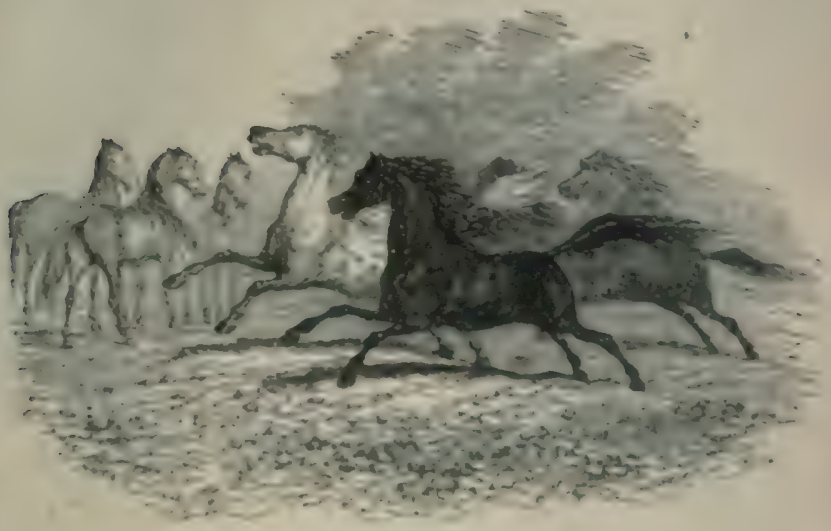

Cuevaus meales.

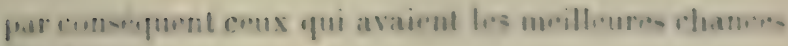
te riussite.

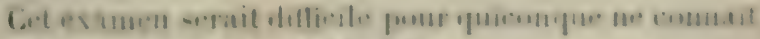

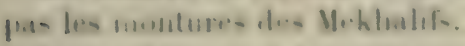

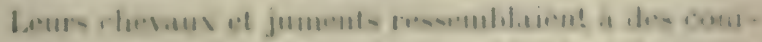

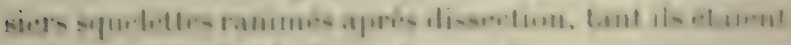

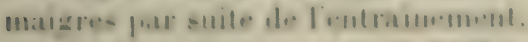

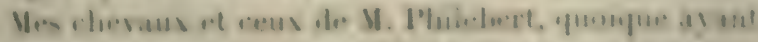

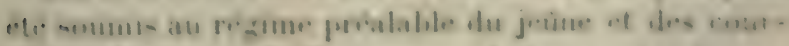

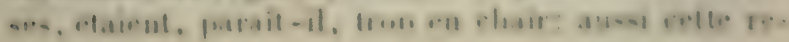


maryue nous était souvent adressée pendant la runte:

- Vos chevaux sont trop gras!

- Ils ne pourront courir assez!

- Ils crèveront de fourbure!

Comme cutle perspective n'avait rien de rassurant, je proponsai à mon compagnon de courir chemin faisant, pou mieux priparer nos chevaux, quelques troupeaux de gazelles que nous apercevions devant nous.

Cette propusition fut acceptée avec enthousiasme.

Le soir, en arrivant à notre bivouac des daias de Rasel-Chàb, nous rapportions chacun deux gazelles que nous avions tuies; elles servirent amplement à notre diner et à celui de notre suite.

Cictle chasse à la gazclle, comme on la fait dans le sud, est très attrayante. Toici comment on s'y prend:

Lorsqu'un troupeau est en rue, le chasseur tàche de le gagner au rent; il s'en rapproche ensuite d̀ une pelite allure; puis, quand il est i six ou sept cents mitres. il lance sur lui son cheval à fond de train et l'approche in une minute ou deux à la distance de soixante à quatrevingts pas... C'est alors que, sans ralentir l'allure, le chasseur tire dans le principal groupe ses deux coups de fusil chargés à balle ou à cherrotines.

Dans cette chasse, on a le double plasir du courre el du tir: quand un bon chasseur en possède l'habitude, il est rare qu'il ne tue pas une ou deux gazelles dans le troupeau couru.

Il y a certainement dilficulté, pour nous Européens, à tirer à cheval au galop; cela tient à ce que nous y sommes bien moins exercés que les Arabes, et à nos selles, qui ne nous isolent pas, comme les leurs, des réactions aux allures vives. Mais aussi quelle satisfaction quand on réussit! 

Cimmo trus les thasseurs, gens a prisates - il on ful. Jeg Mohthalifs augurèrent de nos suevos à venir par la reussite du debut: "La chair apposle la rhair, licairnt-ilson

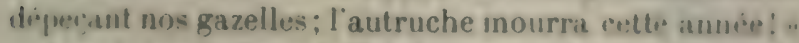

Aux laras de Ras-el-Chicab, nous n'itims qu iो muilii elumin du lieu où nous devions faire motre promiere? stition.

Niuts nous mimes en roule le lendemain, des lianrure. pour gagner la daia EI-Bengra ', distante de laghouat de dix-huit lieues.

Less élaireurs avaient reconnu pris diolle? un ridir dont lean pouvait nous abreuver trois juurs.

Rien de plus pittoresque que l'installation d un hivouac dans une belle daia.

leas chasseurs se dispernent par groupuess sums les plus beaux betumens a l'omlirage (o)uffu.

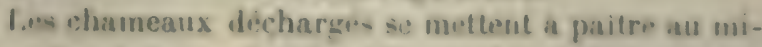
lien du camp, un les vuit allomaer lenr long cou pour Lomidre les plus basses branches des arbres.

I.to cheraus sont attaches a la iurdes, lent ferrur. est bisitibe el assujettie; il $y$ a a cela grande impurtalleses.

1) allume des feux, un propare le ropas.

lies dispositions sont souvent interrompunes par do listres yui se livent et meltent. en se jount an moloss des gens et des bretes, le camp en emoi. - Chacun lom lames yui une poirre, un mallet, un baton, un conp d.

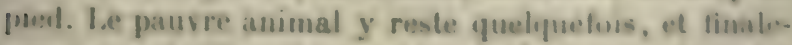
ment va cuire dans la marmite quil avaif qur-lopuefois renverace linstiont d'avant.

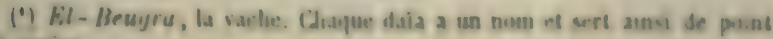
de riprere. 
Enfin on fait son lit, et, apres tous les soins accomplis, les longues causeries du soir terminees, chacun se couche quand vient le sommeil.

Liopération est simple pour les Mekhalifs, le sol est leur duvet habituel.

Nous avions, nous, des hamacs que nous suspendions aux branches des betoums; le moment venu, nous nous y glissions arec la certitude d'y pouvoir dormir sans être dévorés des moustiques, comme à Laghouat.

Il y a deux manières de forcer l'autruche, selon que l'on court le bedou ou le gàd (1).

Dans le bedou, le chasseur doit prendre l'autruche avec le mème cheval, sans relai ni rabatteur; c'est la chasse la plus difficile, celle qui demande le plus de science du courre et les meilleurs chevaux. Elle ne se fait qu'isolément.

Quand les chasseurs sont en nombre, on chasse toujours au gâd. Cette manière, comme le nom l'indique, consiste à se poster à un endroit convenu, près d'un point culminant, d'un arbre élevé, d'où on puisse voir de loin les autruches, que des rabatteurs vont lancer.

Ce qui rend le gâd possible, c'est que les autruches suivent presque toujours la direction qui leur est donnée au moment du lancer. Les rabatteurs qui connaissent la position de l'embuscade se basent sur elle; mais l'opération de diriger ce rapide échassier n'en est pas moins fort difficile et fatigante.

Il faut d'abord explorer le pays, dans un rayon d'un

(') Bedou, 'de beda, a commencé. - Gâd, posté, embusqué. 


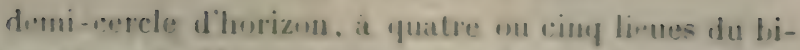
volue pour découvrir les autruelurs: puis, apres les avoir laneses dans la direction roulue, il faut les y maintenir par une toule de rubrigues. - Il faut tenir enfin lis poursuite raide prour lors fatiguer le plus pussilhe.

Aussi ne choisiton que des chasseurs emerites pour rabattre: et il est d'usage chez les Mekhatifs, dan- leurs ringlos sur la chasse a l'autruche, de leur accurder la mitie des dipouilles conquises.

Nous nous trouvions dans les conditions voulues pour chasser au gad.

Il fut convenu que nous formerions deux pelotuns. des quarante courmers que nous étions; que chatque preloten courrait un jour sur quatre, afin que les chosvaux eusint le temps de se repuser.

M. Philebert et moi avions chacun deux chesaux. cela nous permettait de courir tous les deus jeurs: mais dians motre impalience de jouir de celle rhasse, nous tronvions que celait trop peu encore.

Las Arabes, toijours lideles aux tradıtions du pawe, ne mampent famass, quand ils sont en campagne pour la guerre ou la chasse, de se reunir après la pritire de lian t', el de lenir consicil.

1.0s excuements du jour y sont racontés et apprébrive, les fortes tetes y decident des operations du lendemain, apris avoir resurilli les avis, renseigmements at undires ile tous ceux qui ont a en finurnir.

Ces rêtunions du soir, ou chatun geut connmuniq̨uer

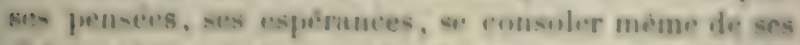
mésalentures, merme en les fanaut partager a laudi-

(1) Guater heures de liapres monds. 
toire, sont les plus intéressantes. - Les plus jeunes, en écoutant le récit des anciens, dont l'expérience et la sagesse sont reconnues, peuvent mettre à profit les leçons de ceux qui ont vu plus qu'eux et fait davantage.

Notre conseil du premier soir fut très animé; on connaissait des autruches sur plusieurs points; chaeun voulut émettre son avis, sa préférence, en déduire le pourquoi et le comment.

Enfin la question importante, celle du lieu d'exploration, ayant été déterminée, il fallut désigner les rabatteurs.

Le choix unanime tomba sur de vrais Mohicans pour la sagacité, Guettaf et Naïmi, dont les prouesses nous étaient connues.

Malgré le profit qui attend les rabatteurs lorsque la chasse est bonne, ceux-ci se font beaucoup prier; car en dehors des difficultés déjà citées, leurs chevaux, trop surmenés, se trouvent souvent indisponibles pour les chasses suivantes.

Les autruches font aussi quelquefois défaut; ou bien, effrayées par une caravane, ou toute autre cause, elles échappent aux rabatteurs. Quand ce contre-temps arrive, c'est une grande mésaventure pour ces infortunés qui rentrent au camp tout marris. Les plaisanteries et les reproches ne leur sont pas ménagés; leur susceptible amour-propre subit un tel assaut, qu'ils jurent par Sidi-Abdallah qu'ils ne feront jamais plus cet ingrat métier.

Il nous fallut donc prodiguer les meilleures amitiés, les flatteries les plus nuancées à Guettaf et Naïmi, pour les décider. 
- Vous ètes des guerdjoumas ${ }^{1}$, , leur dit le cand BelAbbès, vous avez du bonheur, nous faisons choix de vous pour que notre début soit heureux... " Et chacun de renchérir:

"-Vous avez d'excellents chevaux!"

"- Persoune na l'ceil de Naîmi, qui voil à une journée de marche!"

"- ruettaf est notre maltre à tous pour lancer et savoir maintenir l'autruche dans la bonne voie. - II court comme une gazelle! " Et on nous raconta cominent littettaf, un jour, courant une autruche qu'il ne pouvait espérer atteindre avec son cheval trop fatigué, mit pied a terre et acheva de la forcer avec ses propres jainbes.

A un pareil concours d'eloges nus futurs rabatteurs ne tirrent point : ils nous promirent de faire pour le mieux.

Changeant alors leur attilude modeste et incertaine en celle de gens résulus et surs deux-memes, ils nous dirent :

- Il faudra, par Dien, qu'il ne soit pas puused d'autruehes dans le pays pour que demain nous ne rous les amenions dans les jambes. Tenez bien vos Ames!... alvaissez vos yeux surs la terre, du colé du sud-nued. Cest par la que nous viendrons. "

Sur celte assurance on se sépara, chacun fut se purêparer par le repos un la réverie au courre du lendemain.

Habituellement un dort bien dans les dains, leenucoup mienx que dans les maisuns des cuasis, qui gardent, la unit. Ia chaleur qu'elles ont recueillie le jour.

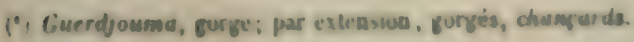


Les nuits des daias sont fraiches et parfumées par les plantes odoriférantes. Elles peurent ètre comparées aux nuits du nouveau monde, si poétiquement décrites par Chateaubriand; car elles en ont le charme, le merveilleux, les beaux clairs de lune et le silence.

Ce n'est pas le seul rapprochement que l'on puisse faire de cette région de l'Algérie arec les savanes de l'Amérique.

Bien des fois, dans le cours de mes chasses dans le Sahra, je me suis figuré ètre un personnage de Cooper et vivre en compagnie des Peaux-Rouges. Le pays, les gens qui m'entouraient, les scènes auxquelles j'assistais et prenais part, étaient celles de la Prairie, moins le vieux trappeur, moins aussi la possibilité d'ètre scalpé.

Quel est le chasseur qui n'aimerait se trouver un jour dans un immense territoire de chasse, affranchi de toute entrave, libre de se mouvoir en tous sens, arec droit de vie et de mort sur cette si grande et si belle variété de gibier, sans que personne songe à le lui contester; libre enfin de pouvoir oublier le souci des affaires pour savourer alternativement la chasse émouvante et la douce flânerie!... Voilà un beau rève !... eh bien, ce rêve devient une réalité dans le Sahra...

En Europe, on ne saurait éprouver ces sensations intenses. La chasse y est soumise à des lois restrictives, à des contraintes sans nombre, à des formalités fastidieuses... Elle est bornée à des territoires très limités; peu d'imprévu, peu de péripéties, - et enfin, rien de cette poésie, de ce vague grandiose que les grandes solitudes seules révèlent au cœur de l'homme.

Loin de moi l'idée d. rléprécier les chasses de France, auxyuelles tant de confrères prennent plaisir; elles sont ce quiuse socićté très agglomérée a pu les faire. 


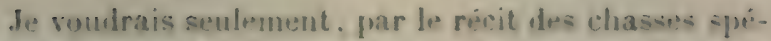
ciales de I. Mererip, ouvrir de nouveaux herizon- a tous for disciples de saint llubert. auxiquels ne sullit plus $\mathrm{L}$. rincolte prérue de quelques lières et perdreaux par. saison.

Mais voici une longue dizression qu'il est temps do finir pour reprendre notre chasse.

Maltree les conditions favorables ou je me trouvaipun une liver au repos, j'ens, comme à la veille diun. muverture de chasse, un sommeil agiti. Je songrai qu. luus mes compaznons, montes sur des chevaux rapintecumme le vent, prenaient des autruches â volonti", tanlis que moi je n'en pouvais joindre aucune. Mon chr.ral punvait a preine galoper ou sabattait des quatre pieds. ynand, a forre de les talonner, il prenait un train plus

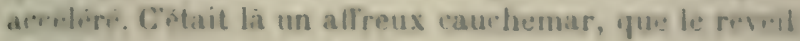
vint heureusement dissiper.

Xous lumes sur pled dés l'aube, quoiqu'il dỉt seconul.r encore bien des heures arant d'aller prendre pusition au lieu désigné.

Xous etions dans une agitation febrile que rien ne ponsait calmer, nous allions souvent caresser nus ithe. vaus, mus ajustions leurs harnais, qua d'exemple d..

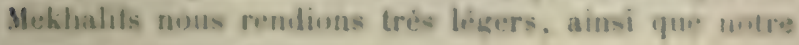
propre accoutrement.

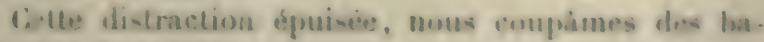
guetles de jujubier sanvage pour stimuler mos chessans au brosin, et pour en aseener un coup sur la mughe de.s antruches que nous equtriuns jemilre.

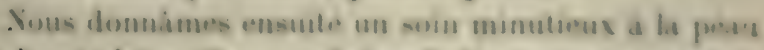
de bouc pleme deau, qui derait etre motre compagnes 
inséparable pendant la durie du courre. - Cette précaution est obligatoire, sous peine de souffrir toutes les tortures d'une soif ardente, que la rapidité de la course et le soleil déterminent.

Le déjeuner nous fit encore gagner quelques instants, mais on mange peu au mois de juillet, et il est plus sage de ne pas se charger l'estomac au moment de courir l'autruche.

Nous étions à bout de patience, quand Bel-Abbẻs et Toumi vinrent nous dire que l'heure approchait, quil fallait abreuver nos chevaux. Chacun de nous s'emfressa de faire boire cinq ou six litres d'eau à sa monlure, contrairement encore aux idées admises en Europe. Ici l'expérience a démontrú l'efficacité du procidé; les chevaux, du reste, sont à jeûn depuis la veille; on les fait boire pour la soif à venir, sans redouter de troubler leur digestion.

A onze heures et demie nous étions à cheval.

I.es Mekhalifs, qui composaient le premier peloton de coureurs, vinrent nous prendre en cérémonie.

Ils formaient un groupe que Callot eût été heureux de rencontrer.

Vètus de pittoresques haillons, n'ayant, afin d'ètre j)lus légers, que l'arçon nu et un feutre pour selle, une ficelle pour tètière de bride, ils avaient au suprème degré l'apparence de vrais gueux.

Ils me prièrent de commencer la marche. C'est un honneur qu'ils rendent au chef ou à celui qu'ils croient le plus heureux.

Ln homme heureux, selon leur croyance, mène ì lien l'entreprise de ceux qu'il précède. Un homme qui a du guignon le communique à qui le suit. Je fis 
mentulement des voux pour ifle lionmm heureux, et nous muss inimes en marohi: en promemçant tous les muts sacramentels en usag., Besm-Allah, - au nom de Dieu!

Vous avions prealablement fnit rentrer lous nos clonmedux et ródeurs dans notre bivouac, afin de ne pas

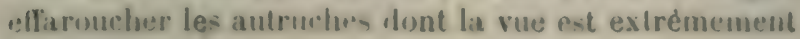
perçante.

Sinus allimes frendre por. sition a desux kilumitres de nutre campement.

Noux mimes pied à terre sulus de grands bolonums du haut desifuels on demisnit le pays.

C'stait là sotre gâd, indique aux raballeurs. Derus Mokhalifs montèront sur les plus hauters branches de larbre le plus elevé, afin de voir venir de loin.

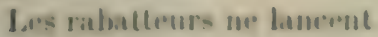

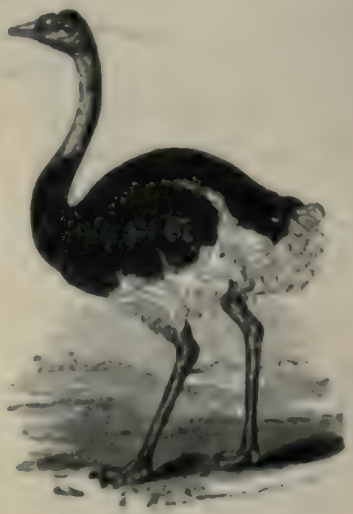

Antruetia. jamais less antruches as and

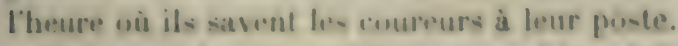

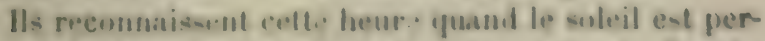

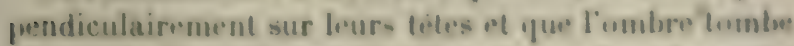

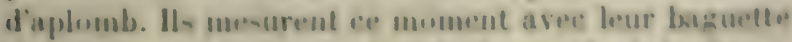
de sedra quäls tienusut verticale. - Guand elle we

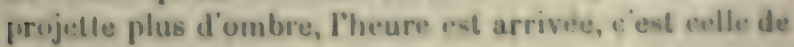
midi.

Nous devions allendre au moins ume demi-loware encore avant liarrivie pussible des autruches. Nutre

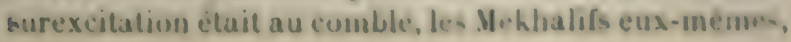


malgré leur gravité habituelle, avaient comme nous la fièvre de l'attente.

Chacun parlait, gesticulait, invoquait son marabout de prédilection, récapitulait les chances favorables.

Le chhili ( ${ }^{1}$ ), par bonne fortune, embrasait l'atmosphère; de fréquentes recommandations étaient adressées aux vedettes.

- Ourrez les yeux, fils de juifs $\left({ }^{2}\right)$ ! leur criait de temps à autre le caïd Bel-Abbès. - Ne vous laissez pas tromper par les autruches! Découvrez-vous bien le pays?

- Oui, n'aie aucune crainte, il est dans notre œil comme dans un miroir; mais cela fatigue de regarder fixement, et nos pieds cuisent sur les branches,

- Posez voś yeux à terre chacun votre tour, cela vous reposera, - patientez. Ne nous jaunissez pas la figure, - il s'agit de votre réputation de chouâfs $\left({ }^{3}\right)$.

Un Mekhalif, vieux chasseur, dans le but de Iromper l'attente générale, proposa de consulter les gorads pour savoir si l'autruche mourrait.

Cette bizarre expérience consiste à prendre des gorads, sorte de poux-de-bois que l'on trouve sur les Egypte.

(अ) On sait le dédain et la haine traditionnelle des Arabes pour les juifs. cette expression fils de juifs est souvent employée par eux quand ils s'exeitent à la guerre, à la cliasse ou même dans les jeux.

Elle a pour but sans doute de réveiller l'énergie, car elle est jetée à lit face cumme un souflet. Celui qu'elle atteint doit alors montrer, par plus de vigueur, qu'il ne la mérite pas.

Une autre version plus favurable à la gent israélite est celle-ci. Les tils d'Israël sont tellemtent délurés et aptes à la réalisation d'un but lucratif, qu'ils montrent pour y parvenir des fualités tout à fait exceptionnelles. C'est àor's à ces qualités qu'il serait fait allusion dans cette qualification de " tils de juifs. $n$

(3) De chouf, voir. - Chouâf, qui voit très loin. 
rheramx mal suigues coux ups Mokhalifs, a ce titre, en retiont alumbamment pourvus.

(In les expuse au soleil, sur la terre, au milieu diun cerile de la dimen-ion de cinquante centimatros de diamètre.

Si l.s gomds sunt cuits avant d'alteinlire la circonferrence da cercle, cest signe, disent nawement l... Mekhalifs. qu il fait chaud et que la temperature m-l propices a la mort des autruches.

sii, all contraire. le gural atteint le hord du cerrle. sans avuir été róti. il fait froid et les chevaux auront irup a faire pour forcer l'aulruche.

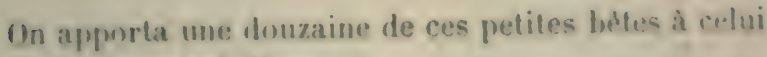
qui avait proposi l'épreuve.

I. humbumuse, avec la conviction et la gravite que lui farai-sait complurter la rirconstaner, aplanit le sul arec le pan do son burnous, trarat un cerrele de la di. mpriniun voulue et mit los goracts au centre.

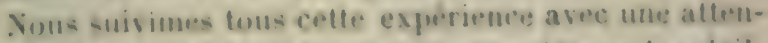
tion estreme. Datorol les gurads, stimules par lesuleil, se mirent en mouvement avee vivacite, et parecururent rapidement la moitie du trajet du centre a la circunference, mais ensulte ils raleulirent leur marche, se tor. dirent, se renverserent sur lo dos, puis restirent immohiles. Ils ftaient litteralement grillo's (').

.-Il fait chaud! - il fait chatud: - l'autruche mourra ! direot comme un seul homane les Mekhalits.

- Oui, liautrache mourra! reprit te canl Bel-Abtris: mais je vais. mes enfinnts, vous faire une recommandation.

(1) II y avait plus de 60 degrós rentigrades an sinteil. 
- Le commandant et l'officier sont nos hôtes, il faut qu'ils prennent des autruches. - Ceux d'entre vous qui arriveront les premiers sur les dolmânes ( $\left.{ }^{1}\right)$ les rabattront sur eux afin qu'ils puissent les joindre et les prendre...

Je l'interrompis et lui dis: - Les prendre, c'est bien ainsi que nous comptons faire, l'officier et moi, mais nous voulons forcer nous-mèmes, sans le secours d'aucun de vous! - Je ne vous demande qu'une chose, c'est de ne pas nous faire concurrence lorsque nous aurons fait choix des autruches que nous courrons! Cela fut convenu ainsi.

Nous terminions à peine cette convention, lorsque les vedettes nous crièrent cette phrase, qui nous fit bondir comme si nous eussions été touchés par une pile électrique!

- 0 mes seigneurs, prenez vos chevaux! l'autruche vient!!!

En un clin d'oeil nous fûmes à cheval, accablant de questions nos vedeltes qui voulaient descendre de leur arbre pour monter aussi leurs chevanx.

- Gombien y en a-t-il?

-De quel côté viennent-elles?

- Sont-elles séparées ou en masse?

- Sont-elles près ou loin?

-Y a-t-il beaucoup de mâles?

- Viennent-elles droit sur nous?...

— Ne descendez pas, fils de Satan! renseignez-nous bien, nous ne partirons pas sans vous.

Les vedettes, peu rassurées sur ce dernier point, el pour cause, trépignaient sur leurs branches en répon-

(') Pluriel de dolime, mâle de l'autruche. 
dinul : - "Oui! - mon! - mlles sont pros. - II y.m a Irois - ciny - huit - douzo - seize! - Filloo arrivent ensemble!... Mltende\%-nous pesur lianour 1. biru! si vous partez sans nous, jamais nous ne rommul.rons sur liarbre!..."

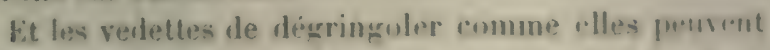
de leur perehoir. en laissant anx asperilis do lamfwans de leurs vètements!

lirimpess sur nos selles ou debout sur nos idri-r. mons aperoevons bientiol umus-memes les autrumber 'pui viennent droit å nous!

Impussible de peindre l'omotion qui nous sai-it daus ce moment: nos figures s̈illuminont, nos yeux laureut des edairs, nos membres sunt agites de momominls forveux dont se ressentent nos chevaux, qui se cabr.ut et bondissent !...

Chacun veut innner un rensegil :

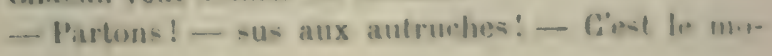
ment!

- Non, pas encore! - Mltenitez: - Raballun-. P. a ganuebe! - Nun, a droite! - Mlendez! allenter......

Mais, recommandaliun vaine: le demon du mous.. ment l'emporte; ef au premier fras en avant fait par un rhasorur, toute la bande s'envole comme une nun.e des -aulerelles et court droit aux autruches.

cielles-ci nous apercoivent anssitot et font un a Iroile ?... Nous les avions alors a cinq cents metres de nous 1

Ces unguilique oiseau, surtunt quand il est en truapm.

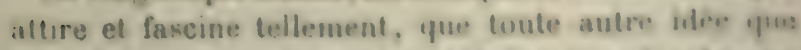

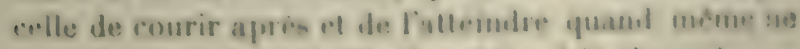
anmait entrer dans la lete de celui yua de thane: 
Toute préoccupation de conservation personnelle et de celle du cheval disparait.

La possibilité de s'égarer, chose assurément fort grave dans ces solitudes, ne touche point.

On n'a plus qu'un unique objectif !... ce grand échassier, aux plumes onduleuses et chatoyantes, qui fuit i lire de jambes... et que l'on veut atteindre à tout prix !

- "L'autruche tire le cour et l'œil ", dit-on dans le Sahra.

Sous l'empire de cette irrésistible attraction, nous courions avec frénésie! - Des gens calmes qui nous auraient vus passer dans ce moment nous auraient certainement pris pour des possédés, faisant une de ces chasses fantastiques des légendes d'autrefois !...

Les autruches avaient encore augmenté leur allure à notre vue, de sorte que nous ne pûmes gagner sur elles. - Au bout de deux ou trois minutes, nous perdimes mème du terrain, à notre grand crève-cœur, et force nous fut de redonner l'haleine ì nos chevaux en modérant leur vitesse.

Je profitai de ce moment pour jeter un coup d'oil sur mes compagnons.

J'étais dans le premier tiers des coureurs les plus avancés, celui des enthousiastes!

Le second tiers, qui venait après nous, était, comme je le reconnus ensuite, celui des rusés, qui ménageaient prudemment le fond de leurs chevaux.

Venaient enfin ceux dont les montures ne pouvaient faire mieux. Parmi ces derniers je vis M. Philebert, qui s'escrimait des jambes, de la bride el de la houssine, pour faire rattraper à son cheval la distance perdue; mais il me parut que ses efforts seraient en pure perte. 
Nous reprimes bientît la mime allure quiau départ: il ure fitliait pas perdre de vue les autruches. Nous ga-

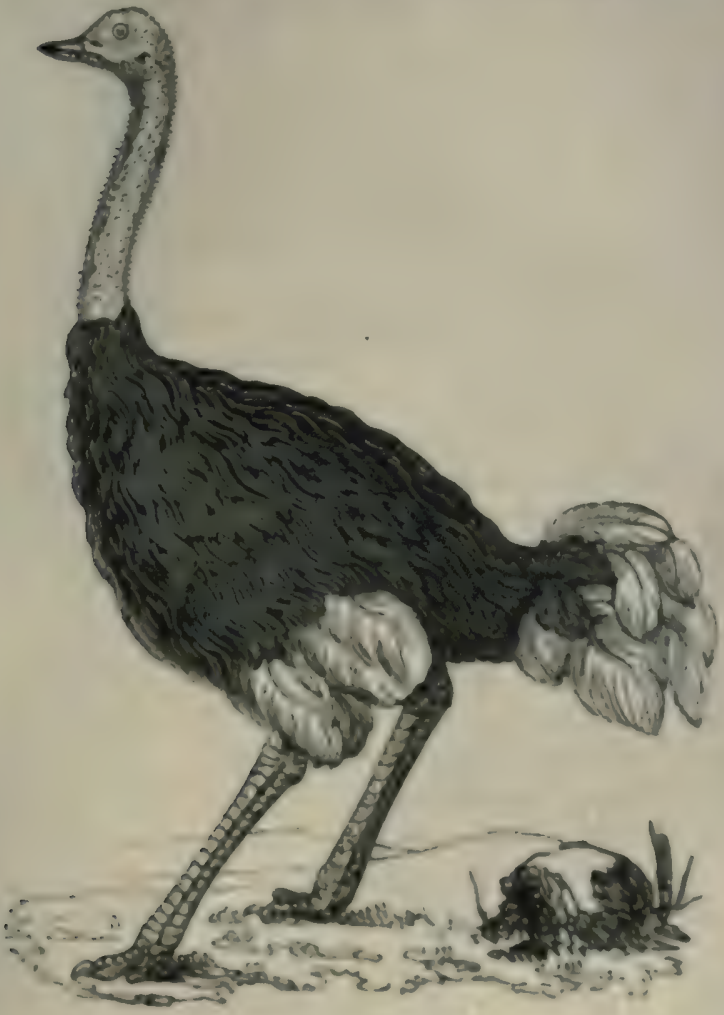

L'autreche noire.

guimes sur elles. l'uis, l'extrime vitesse de nos cherbas se ralentisant, elles reprirent leur asantage.

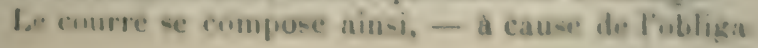


tion de redonner de l'haleine aux chevaux et de maintenir leur fond, - d'alternatives qui rapprochent et ¿qui éloignnent, jusqu'au moment où, étant forcée, l'auIruche sarrète d'elle-mème, souvent à la distance d'un kilomètre du chasseur $\left(^{1}\right)$.

Tant que les autruches courent réunies, les chassintur: ne forment qu'un groupe; mais lorsqu'elles sentent qui elles seront bientût forcées, un suprème instinct leporte i se disperser, afin d'augmenter leurs chances de salut.

Chacun prend, à ce moment, la piste de la siente.

Un suit pour ce choix l'ordre dans lequel on est placé, c'est-à-dire que les autruches qui se détachent à droite sont suivies par les coureurs de droite; celles: qui vont à gauche, par ceux qui se trouvent à gauche, et toujours ainsi en se fractionnant individuellement.

Nos autruches ne se séparèrent que quarante minutes environ après notre relancer; elles se dispersèrent en éventail.

C'est alors que je vis les coureurs du second tiernous rejoindre, nous dépasser, et chacun d'eux prendre la direction de l'autruche qu'il avait devant lui.

Nous fimes tous de mème, - je parle de ceux qui étaient restés les premiers en ligne, car presque tout le dernier tiers ne put rejoindre.

Yous continuâmes ainsi à suivre nos autruches, qui étaient alors à huit cents mètres à peu près de nous.

J'en avais une fort belle devant moi, qui me paraissait ètre un mâle, tant elle était noire.

(') Si l'autruche, comme la gazelle et bien d'autres amimaux, reprenait haleine dans sa course quand elle est poursuivie, on ne parviendrait pas, je erois, à la f rcer avec les moyens employés; mais du moment où elle est Jancée jusqu'ì celui où elle succombe, elle lournit sa traite avec la mêtméraideur et se crève positivement elle - même 
Jo purtis himblit mes compagnons de vue pour no phus un uremper que de ma chase. - Je navais pas tmin tumene mon cheval, je vis bientit que jarais chance de succès.

Apres quelyues minutes de grande allure, je gagnai som-iblement sur mon autrurhe. - Ses ailes commencirent a frondro le long de ses cuisses, ce quai ent un indice de fatizue; ses pieds soulevaient la prousiere en raclant lat terre.

Jetais dans un ravissement a nulautre pareil. Jexritai mon cheval, qui se ranimait d. lui-mene en vovant se rapprocher la distance qui le siparait de notre bite. Je fe carresai en lui faisant de heaux compliments. - Je poussai des cris impusibles et entunati, je ne sais sur yuel air, ni avec quelles paroles... un ehaut de triomples !

Quiromplue se rappellera te plaisir quil a eprouse on luant. prour la première fuis, a la chasse, un lievre ou un perdrean, comprendra - par comparaisun - - ha joe qu m'inondait lorsque jalteignis mon autruche. de ur puss la dectire, il faut leprouser.

La paure hóle s'elait arrelese â mon approche; clle me pourait plus avancer, elle avait te bee grand ouvert et racillatit sur ses palles.

de melancai a terre puur la prendre par le cou. J. roulais létreindre dans mes bras pour en tien promire possession. Au moment ui je la saisi-ais, jentendis derriere moi te galop d'un cheval et quelqu'un qui me criait :

" Prends garde aux coups de pieds. Srappe a la Letel to

J. me relournai sans làcher un proie, et je vis B..I-

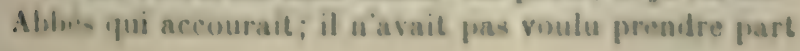


à la chasse et m'avait suivi dans la crainte de me voir m'égarer.

C'était une précaution superflue ce jour-là, mais dont je lui sus bon gré néanmoins.

Il mit pied à terre, et, après avoir abattu l'autruche d'un coup de baguette sur la tête, il se mit à la saigner ( $\left.{ }^{(}\right)$.

Pendant qu'il se livrait à cette opération, je prenais ma peau de bouc, je rafraîchissais avec son contenu la bouche, les naseaux et le ventre de mon cheval.

Ce bon animal se prètait avec une grande satisfaction à cette attention, que les Mekhalifs ne négligent jamais; puis je bus un peu moi-même pour ramener la salivation que la rapidité de la course et la grande chaleur avaient arrêtée en me desséchant la bouche et le gosier.

J'aidai ensuite Bel-Abbès à dépouiller l'autruche, ce qui se fait en coupant la peau au-dessus des cuisses, région où cessent les plumes; on enlève seulement ainsi la peau du dos, des ailes et du cou.

Je fus un peu désappointé de ne retrouver dans mon autruche qu'une belle femelle, au lieu d'un mâle que j'avais espéré et cru reconnaitre de loin. Nous la retournâmes sur le dos, les pieds en l'air.

Après l'avoir entourée d'arbustes et lui avoir mis quelques plumes blanches sous les ongles pour effrayer les carnassiers, nous reprimes le chemin du bivouac, d'où nous devions envoyer des chameaux pour recueillir les victimes et les rapporter le soir même.

(') On sait que les musulmans ne mangent que la chair des animaux qu'ils ont saignés en les tournant vers la Mecque et en prononçant la formule de "Au nom de Dieu, Dieu seul est grand. „Cet usage rituel est en commémoration du sacrifice d'Abraham. 
A re moment nous fümes rejoints par M. Phileliert, qui, malgre le peu de vitesse de sign cheval, navait pras voulu abaulonner la parlie.

II avait suivi nos trares en subissant en partie th réalité de mon rève de la nuit prếcédente. - Il stimulait toujours sa monture arec le méme acharmement, mais sans en tirer autre chose quin lourd salop, sur place.

II était exaspéré de sa mésaventure, il jurait comme un païen après son quidar (') el contre whii qui le lui avait sendu pour un coureur de fond.

Je comprenais, je ne dirai proint sun désappointement, car ce mot ne rendrait pas las situafirol dans larguedle il se tronvail : elle approchait du désespoir.

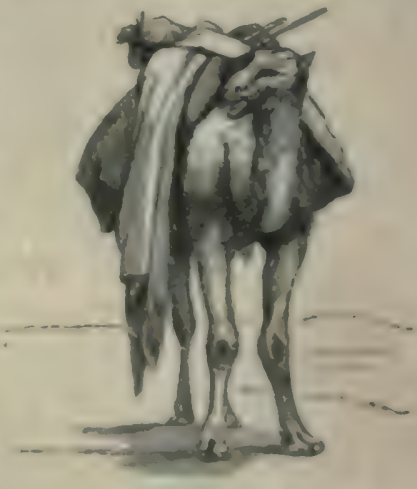

La chameas ourteup.

Nos condoléances le Lunchaient doulant mons. qu'à charque instant nous trouvuns sur notre parcours nus cumpagnons les Moklaalifs qui revenaient avee des dépouilles.

Less seize autruches avaient suceomlne:...

I notre arrive au bivouar. unus fimes acrlamés par tuus ceux qui n'avaient pas pris part a la chasse du jour. - Ils nous dirent: "Dieu vous donne la sante

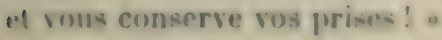

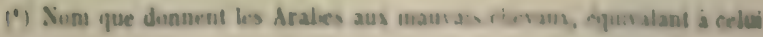
de mor 
Des chameliers furent immédiatement expédiés avec des indications suffisantes pour retrouver les autruches.

On étala leurs dépouilles à l'ombre et, après les avoir netlurées, on les courrit de sel.

Nos cheviux furent l'objet de tous nos soins; on les fit boire à leur soif, on leur laissa brouter l'herbe de la daïa, et peu après on leur donna l'orge $\left({ }^{1}\right)$.

En attendant nos autruches, nous nous inimes à examiner leurs belles peaux; il y en avait six de mâles et dix de femelles.

Après le plaisir de chasser, voir son gibier est le complément de satisfaction le plus agréable.

Les Mekhalifs, qui pensent de mème, faisaient en connaisseurs l'éloge de telle ou telle dépouille : l'une était remarquable par la beauté des plumes blanches des ailes; l'autre, par les plumes d'un noir de jais du dos et du col; une autre enfin, réunissant la beauté des deux coùleurs, était parfaite et n'avait pas de prix.

Et, à chaque éloge, ils répétaient: "Que Dieu bénisse et préserve du mauvais œil $\left({ }^{2}\right)$ ! ”

Chaque chasseur se mit ensuite à raconter les ép sodes de sa chasse particulière : - celui-ci avait été jusqu'a tel endroit, les sangles de sa selle s'étaient rompues, il avait dû les renouer tout en courant.

Le cheval de tel autre avait buté dans une ville de rats $\left.{ }^{3}\right)$ et avait fait panache sur lui; il était remonté malgré cela et avait forcé son autruche, etc.

(') On attache une grande importance à ce que le clieval qui a couru urine et se secoue dans les deux lieures qui suivent le courre; flus tòt il accomplit celte fonction et mieux cela vaut. Aussi est-ce la question préalable entre chasseurs qui s'abordent après le courre. Si la réponse ist affirmative on se réjouit, parce qu'il n'y a pas de mauvaises suites à redouter.

(2) Voir la note de la page 16 de la chasse au lion.

$\left.{ }^{3}\right)$ Medinet-el-firan. Ce sont des gerboises et des rats zébrés qui, pour 
Bientôt ces vastes récipients chantèrent, en bouillonnant à la ronde, une mélodie qui n'était pas sans charmes pour les estomacs des Mekhalifs, - et pourquoi ne dirions-nous pas des nôtres? - Le déjeuner avait éti insignifiant, la chasse et le succès avaient aiguisé chez. nous l'appétit proverbial du chasseur.

Nous fìmes donc confectionner, en attendant la cuisson du hammoum $\left({ }^{1}\right)$, des carbonnades avec un râble d'autruche :- cette préparation si simple, assaisonnée de sel et de poivre, fut trouvée excellente.

Isa chair de l'autruche a la plus grande analogie avec oelle du bouf.

Pendant toute la durée de la chasse, nous avons répété les expériences sous forme de consommés, daubes, biftecks, et toujours il nous a semblé manger de bon boeuf.

I'ne réminiscence de la grande époque gastronomique romaine nous fit songer à consommer, pour la rareté du fait, un plat de cervelles d'autruche.

Les Mekhalifs nous offrirent gracieusement en hommage toutes celles des leurs.

Notre cuisinier, les ayant réunies, en fit un ragoût avec de la moelle des tibias et force épices. - Ce mets rare, tant par son mérite réel que par les souvenirs qu'il rappelait, nous sembla délicieux.

(') Le hammoum est la chair de l'autruche coupée en menus morceau et euits dans la graisse de celle-ci. Cette préparation est savoureuse et très nourrissante.

Les Mekhalifs disent que la chair de l'autruche tient longlemps au ventre, el lui en font un mérite de plus.

Ils ajoutent que l'on peut en manger beaucoup impunément, qu'elle maintient le corps en bon état et la bouche fraiche; qu'on ne peut en dire autant de la chair de gazelle qui, plus succulente, échauffe davantage et rend la bouche amère.

Je laisse la responsabilité de cette opinion à ses auteurs. 


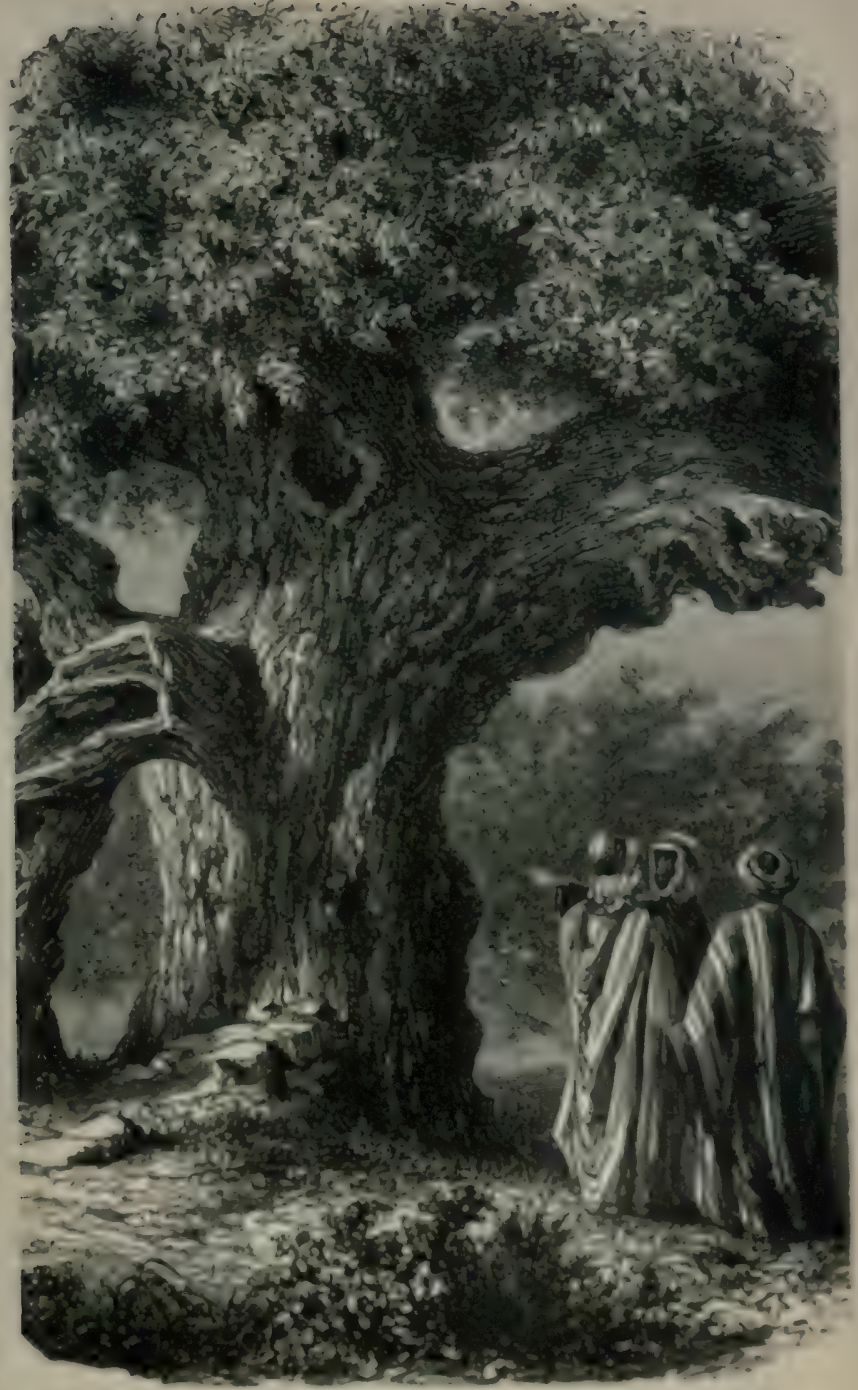

Trune de terntugateve botuum! 



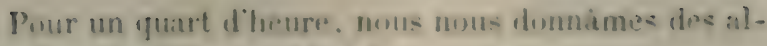
lures de Lucullus el d'Apions.

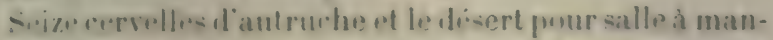
ger :... Nistait-ce pas la du faste et de la magnificonce?

I.es Mrokhatifs conservent avec suin la graisse de l'auIrueder qu ils renferment dans la peau des enisses et itu cou.

Pour mux, cest un spícilique propure à guérir tuutes les maladies intopnes et externes.

Ils ne se font faute de l'imployer et affirment sien tmouver bien.

La chair qui nest pas convertie en hammoum e-t

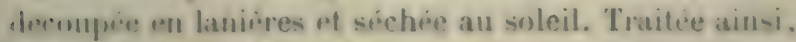
clle sie conserve des mois entirers.

Tout est hou, eout sert dans lautruche : les plumes -e vemulent, la chair se mange, la peau sot de requpentit la sritiose, qui guérit tous les maus; la plante des pieds sert a lace des semelles do brodequius pour less

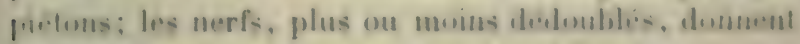
un fil trîs re-i-tant, propre a condre le cuir.

Ausa tes Mrkhatifs ont lhabitude de dire, quaund ils font unn afliare tris avantageuse : "C"est comme liaufruche, plumes et grainse."

Vintre ripus apres celle lexureuse jourufe fut parfait.

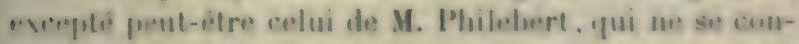
colcit guere de linsureces de son debut. Nais je me hate de dire que nous ne eumes pas à unus apuloner longt.mp.

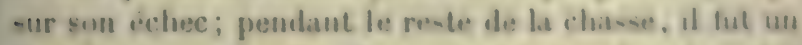
des plus heureux : il prit avee son autre rheval quatre Leaus males , dont les plumes firent notre admiratum.

te temdemain, mons fiomes mareher of trolter devant nons les ehe vaux qui avauent resuru, peur vour dans yqued etat ils se trouvaient 
Deux étaient boiteux pour longtemps, trois l'étaient moins et pouvaient continuer la chasse, un enfin était fourbu.

Ce sont là des accidents presque inévitables, vu les conditions dans lesquelles on est obligé de courir.

Le sol est très rocailleux dans certaines parties; l'autruche semble faire exprès de choisir celles-ci, quoiqu'elle soit la première à en pâtir et à user ses pieds au cuntact des cailloux tranchants, - en quoi elle me parait bien mériter la réputation de bétise qui lui est généralement dévolue.

Aussi proclame-t-on trois fois heureux ceux dont les chevaux se maintiennent sans accident et peuvent fournir les sept ou huit courres de la saison.

Les chasses qui suivirent celle que je viens de raconter y ressemblent tellement que, pour ne pas me répéter, je ne citerai que le nombre des autruches forcées dans chacune d'elles.

Après chaque courre, nous changions de campement, parcourant les plus belles daïas de notre Sahra.

Nous vagabondâmes ainsi, en pleine liberté, environ trois semaines, chasisant, en dehors de l'autruche, pour assurer notre subsistance quotidienne, des gazelles, outardes, lièvres, perdrix gangas; en un mot, tout ce qui nous tentait.

Pour varier encore nos plaisirs, nous entremêlions ces chasses de petites fètes, daus lesquelles nous organisions des tirs à la cible, des courses à pied et des courses à dromadaires pour nos chameliers.

Le jeu de balle surtout était notre récréation favorite.

Les Mekhalifs prenaient part à tous ces jeux avec une expansion que j'ai rarement retrouvée chez les autres Arabes. 
De colte vie journalière en commun s'itait digagée une surte diémulation qui nous entrainait tous à imiter ce que nous trouvions de bien les uns chez les antres. Ainsi nous tenions tête aux Mrkhalifs dans leurs flus rudes exercices a cheval et a pied; par contre, ceux-ri voulitient en faire autant dans la gymnastique et surtunt dans le tir, oi ils avaient des pretentions.

lin jour, pour bif n Irancher cette question d'adre-ce au tir à balle do pied ferme sur un but fixe, - car ils reconnaisaient motre supuriorité pour le tir de vitusse à pied ou a cheval, - nous fimes un concours dans les conditions suivantes.

Il s'agisail de ca-sir un teuf de proule, suspendu à un fil contre be tronc d'un betoum, a la distance de cent pars.

Pour faire la partie plus égale, nous avions dit aux Morkhalifs:

"Vous etes vinet lireurs, uness summes dens : tirez. chacoun ciny baltes sur le but, nous en ferons autant, et 1. parti qui touchem davantage aura gagné. on

Its deevaient tirer cent balles contre nous dix; mais mos armes nous semblationt meillenres.

L'enjeu etait un peau drautruche pour nous, si mos gagnions, ou sa valeur punur les Mrklualife, si nous perdions.

Les chuses ainsi convenues, le tir commença.

Nous en fimes les homeurs aux Mekhalifs, qui paraissaient ne pas douter du sueces.

Ils tirerent succussivement leurs cing balles chacun, mais en vain : aucun n'alteigmt l'ceuf, qui, je dois I avouer, se voyait à peine. - Javais aumai ehois. ce trut à cause de la jactance de leurs melleurs ureurs, qui pretendaient ne tuer le gibier quien lui mettant la balle dans l'ail. 
Quand ce fut notre tour, à M. Philebert et à moi, de tirer, l'attention de tous redoubla. Nos premiers coups, sans toucher, portèrent beaucoup plus près du but, ce yui fint franchement reconnu. A ma quatrième balle, l'ouf vola en éclats.

L'effet fut dautant plus grand que la conviction de tous, après expérience, était qu'on ne pouvaitl'atteindre.

"Tu as gagné la peau d'autruche, me dirent les Mekhalifs, et de plus, nous reconnaissons que rous ètes nos maîtres, mème dans le tir posé. »

J'eus, quelques jours après, l'occasion de les confirmer encore dans cette idée par un de ces hasards qui font sensation quand ils se produisent.

Nous changions de campement et marchions en goum vers notre nouveau birouac, quand nous découvrimes dans le lointain un troupeau de gazelles d'une soixantaine de têtes.

Les Mekhalifs, auxquels j'avais parlé de la longue portée de ma carabine de Lancastre dont ils connaissaient déjà la précision, me dirent :

"Certes, ton fusil ne porterait pas ses balles à ce troupeau. "

Je trouvai effectivement que la distance était considérable; mais comme il y avait un semblant de défi dans leurs paroles, je voulus le braver, et je répondis que je n'en doutais pas. Il y eut sur leurs lèvres un sourire qui protestait mentalement contre cette assertion. Pour le faire cesser, j'arrêtai mon cheval, j'ajustai le troupeau, en visant au-dessus de la hausse, qui portait déjà mille mètres, et, convaincu du reste que ma balle ne s'écarterait pas beaucoup du point visé, je tis feu... On sait qu'une balle de carabine met trois ou 
qualre secondes pour parcosurit un pareil tanjet. i) ija

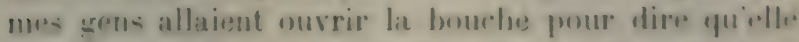
ne parviendrait pas. Lorsque elle fraplat au milieu du troupman en soulevant un prelit nuatge de punsiore.

Cie fut dabord de la sluperur et de libahi-sement. ynii se Iraduisirent ensuite par los exclamation- leplus variées.

L. frumpau de gazplles avait pris la fuite yuand la balle avait frappé, mais jegmorais s'il y avait "ll une het. d'afteinte. Jenvoyai deux ravaliers s'en as-ur.r. Cema-ri partirent au pelit trot sans le moindre espuig: mais yuand ils furent aux trois puarts du chemin, numl.- vimes prendre le galop en agitant leurs burnoms. pull ipres mettre pied a terre et se baisser comme puar ramil-ser qurlıue chose...

fösat, pour comble de l'etonmemont, - aufued je. pris part moi-mème celte fois, - une gazelle qui avait

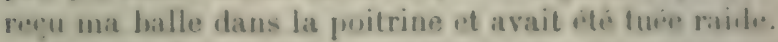

C. enup fut trouve merveilleus par les MekhalifIls vomlurent sfance tenante en perputuer le somsenir.

l'uur cela, ils rassemblierent ume grande quantite de pierres dont ils batirent un realjam th, puis its allierent en chablir un autre parroil a lemolenit ou mat balle asaul frappe la gazclle.

Je fus eurioux de mestrer la distance qui sipumal ces deux points.

II y aritit onze cents meitres :...

I.to Aralues appellent aujourilhui cos doux tumulus,

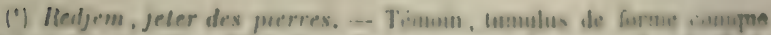

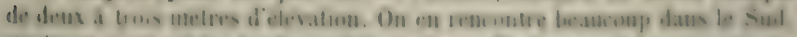

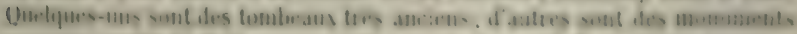

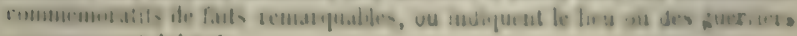
un ponom ont eles lues. 
ıpui se trouvent près de l'oued Nili : Redjrm-el-Ghezal, - témoins de la gazelle.

Quels bons souvenirs j'évoque là!... Je m'y complais peut-être un peu; mais qui n'en ferait autant à ma place?

Quelques orages qui eurent lieu vers le 1 å juillet nous-rendirent celte existence facile par la grande quantité d'eau qu'ils amenèrent dans les r'dirs de la région où nous chassions.

Sans celte bonne fortune, peu ordinaire dans cette saison, nous aurions dû faire venir notre eau, soit de Jaghouat ou du Mzab, ce qui aurait obligé nos chameaux à un trajet de quarante lieues, aller et retour.

Voici le résultat exact des chasses de la première année:

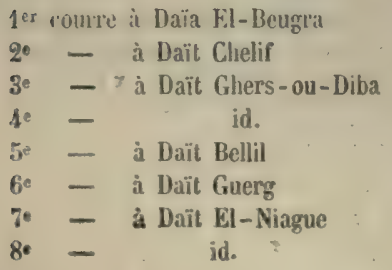

Total. . .
16 autiuches, dont 6 mailes.

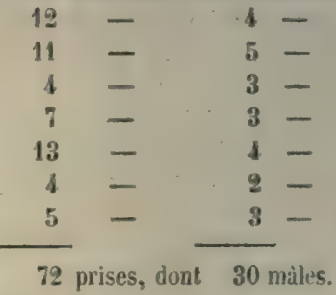

Toutes nos chasses ne furent pas sans quelques déceptions.

Ainsi nous fimes trois fois le pied de grue au gàd: la première, parce que les autruches parvinrent à se dérober aux rabatteurs; les deux autres, parce que ceux-ci n'en trouvèrent pas, et que, selon leur expres sion, "il n'en était pas poussé dans le pays. " 
In jumr au-i yüil faisait fruid, comparativement. nous courimos vainemont une bambe te dix-neuf au-

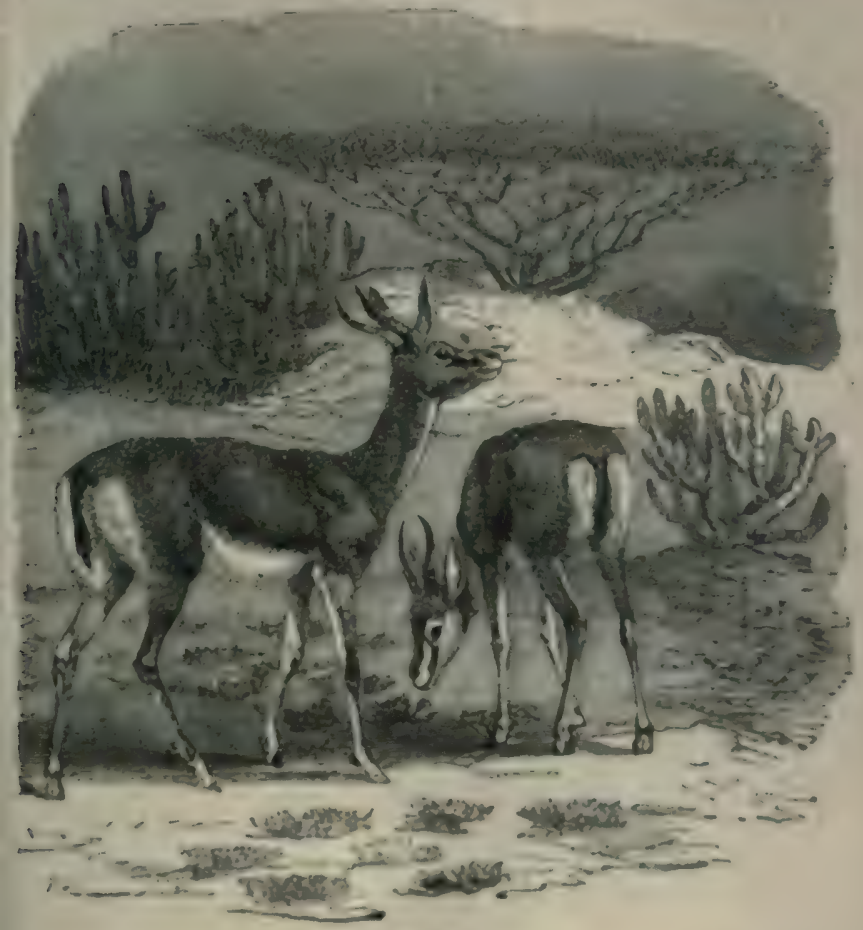

limanties.

Irurhes, qui mons avail defie lance de trop prois par les rabulteurs.

Suns nous efiuns fromis. on allant nums pouster et en mous apercevant yue la journeve nelant pass ansere

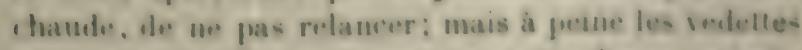
nous eurout-ellos cric yue les aulru-bes venatent. 
qu'oubliant nos bonnes résolutions, nous nous mìmes en chasse, plus forcenés que jamais.

Après deux heures de la course habituelle, la bande ne s'était pas encore séparée: elle avait rencontré dans sa fuite une compagnie de ringt-deux autruches. Ces dernières, étant toutes fraiches et reposées, emmentrẹt les autres en leur communiquant une nouvelle vigueur.

Le dépit de voir un si grand troupeau nous échapper sans nous laisser la moindre plume nous fit continuer notre poursuite contre toute prudence.

Mais, après une course insensée, nos chevaux étant à bout de souffle et de jambes, force fut d'arrèter. Nous avions parenuru un trajet de douze à quatorze lienes!... Nous n’étions plus que denx, le caidd Toumi et moi.

M. Philebert, qui avait apuryé it droite, était de son côté resté arec deux Mekhalif́. Tous les autres, soit que leurs chevaux n'eussent pu suivre, soit que, mieux arisés que nous, ils se fuscent arrètés à temps, araient rejoint le birouac en reconnaissant l'inutilité de pousser plus loin.

Le hasard avait dirigé ce jour-là notre course dans une région sans daïas, sans accident de terrain reconnaissable, en un mot, sans le moindre repère pour nous guider au retour.

Nous pouvions nous égarer et errer pendant le reste. du jour et, de la nuit, sans une goutte d'eau pour humecter nos gosiers desséchés. Nous avions vidé nos peaux de bouc pendant la course pour rafraichir la bouche de nos chevaux. - La situation était critique.

Nous essayâmes d'accélérer notre retour au bivouac en nous guidant sur le solêil, qui était encore sur l'ho- 
rizun: mais mus chevaux refusirent de fromire une

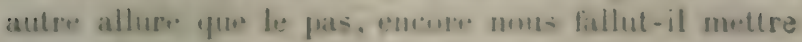
pird a terre pour les faire matrher.

Quand la nuit vint nous premiles, il mus re-tait en-

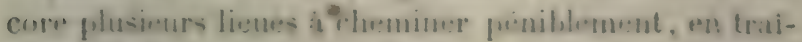
namt nus clervaus par la loribr.

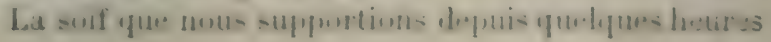

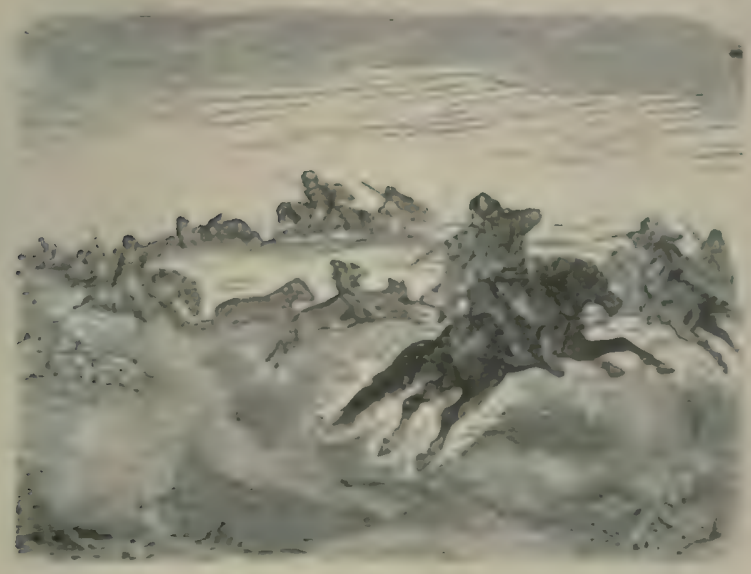

Cheme do la gerollo au galopo

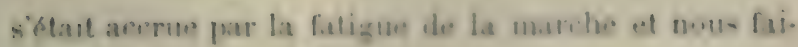
sait eruellement souftrir.

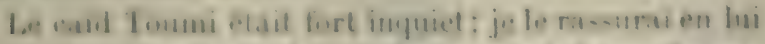

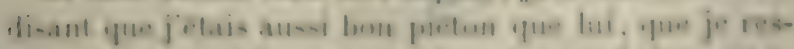

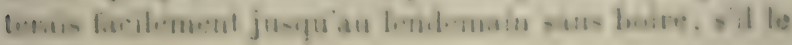
fallait.

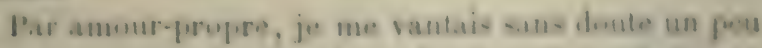

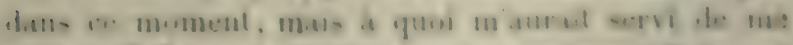

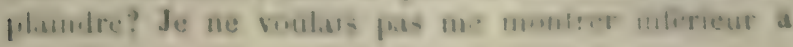


mon compagnon d'infortune lorsquil s'agissait de supporter une dure privation et une grande fatigue, choses dans lesquelles les Arabes n'ont que trop de lendance à se croire supérieurs aux Européens.

Nous continuâmes donc à avancer en faisant marcher nos chevaux devant nous, après leur avoir úté la bride pour les soulager encore.

L'émoi, pendant ce temps, s'était emparé de tous les Mekhalifs qui étaient restés au bivouac, et de ceux qui l'avaient rejoint après nous avoir quittés.

Ceurx-ci furent questionnés pour savoir la direction que nous avions suivie, - si nous étions loin, - si nous avions de l'eau. etc.

Mais lorsque, vers huit heures du soir, il ny eut pas apparence de notre retour, l'inquiétude de tous fut à son comble.

Le caid Bel-Abbès, qui redoutait un accident grave, s’arrachait la barbe. - "Que dira-t-on de nous, s'écriait-il, si le commandant et l'officier s'égarent et meurent de soif, eux et leurs chevaux, lorsqu'ils se sont confiés à nous? Allons, tout le monde à cheral avec des outres pleines d'eau! - Dispersons-nous dans la direction du sud-ouest qui est celle qu'ils ont prise. Que personne ne revienne avant de les avoir retrouvés! - C'est ce chien de Toumi qui est cause de tout ceci: il a entrainé la chasse au lıeu de la rompre, comme il aurait dú le faire, puisque la journée était froide. "

Ce pauvre Toumi ne méritait pas seul ces reproches, nous étions au moins aussi coupables que lui.

Ce fut le caïd Bel-Abbès, accompagné de deux des siens, qui nous rencontra le premier. Nous avions enfin entendu son appel, qu'il lançait de temps à autre dans 


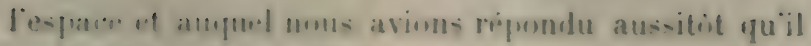
abaif ete a molre jurtoes.

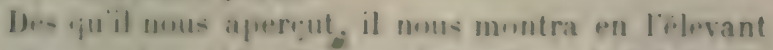

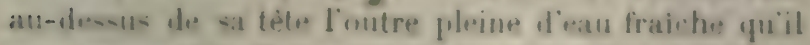

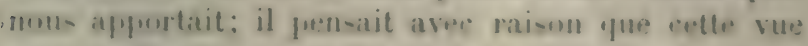

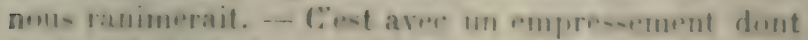

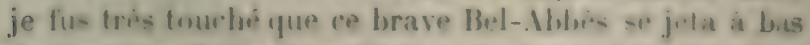

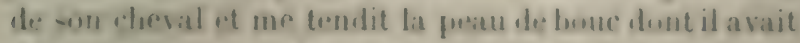
d.ja envert te goulut. a Tiens, mois: " me dit-il. -

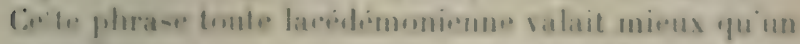

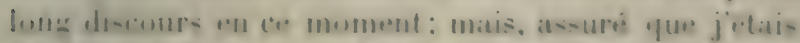
darriver loientid a mos tentes. jo tins a me montreg s-1еjue ju-fui au bout : je le remerciai en lui disant que

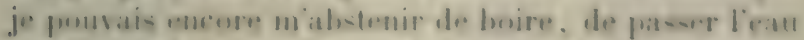

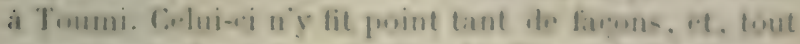

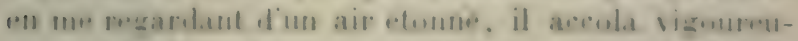

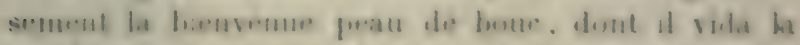

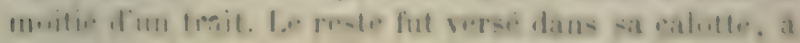
nos chevaus.

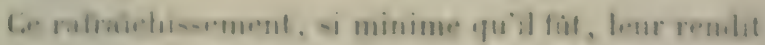

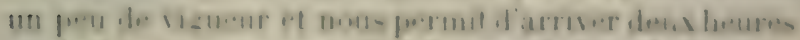

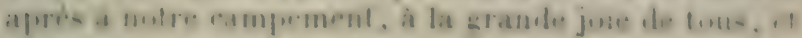
partarulinement a la notre.

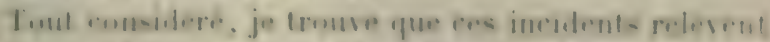

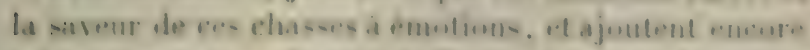
it leur piquant. - Je sus de l'avis de cens qui geon-ent

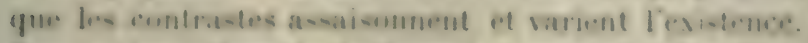

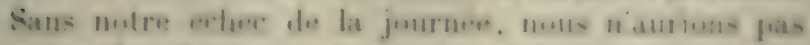

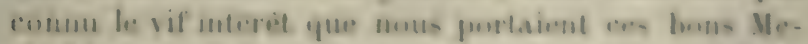
hhalifo: mons riaurions pare pprouse la grande sale-

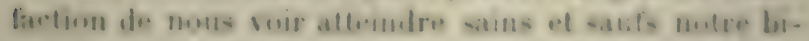

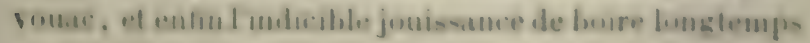
putur apaiser notre soif. 
Tout a un terme en ce monde, surtout les meilleures choses.

Il nous fallut rejoindre nos postes, mais non sans jeter un regard de regret à la région où nous avions passé de si heureux jours.

Dans notre réunion des adieux, les Mekhalifs nous donnèrent rendez-rous pour recommencer la chasse l'année prochaine. "Nous aurons plus de chasseurs que cette année, nous dirent-ils; les plumes se vendent cher, c'est le cas d'en faire de bonnes récoltes. "

Effectivement, jamais ils n'avaient vendu les leurs un aussi grand prix.

Des indigènes, des juifs d'Alger, de Tunis et de Tripoli, étaient en nombre dans le Mzab, à attendre les dépouilles que les chasseurs y apportent tous les ans.

Ils achetèrent les peaux des mâles à raison de $170 \mathrm{fr}$. chaque, avec l'obligation de prendre en même temps deux peaux de femelle pour une de mâle.

Le nombre des peaux de mâle fut augmenté par une petite supercherie que les Mekhalifs ne se font aucun scrupule de pratiquer vis-à-vis des juifs peu connaisseurs.

Il y avait dans le nombre des peaux de femelle dont les plumes étaient presque noires $\left({ }^{1}\right)$, il les déguisèrent facilement en dépouilles de mâle par l'adjonction de quelques belles plumes de ceux-ci. "C'est une bonne plaisanterie, racontail le Mekhalif qui avait réalisé cette opération; Dieu a aveuglé ce chien de juif, qui non seulement n'a pas chicané pour les peaux, mais qui de plus m'a donné un cadeau pour que je lui accorde la préférence. "

(') Celles des femelles sont grises ordinairement. 


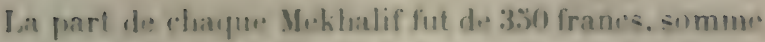

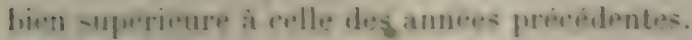

Vous empurlames. M. Philelwert et moi. chacun quatre dopmuilles des autruhes que nous avions forres. Les rabatteurs furent, bien entendu, convenablement rémunérés.

- Vous rmus avez prorté bonheur, nous dirent BedAblope tot Touni en prenant congé de nous; lan joruchain, si Dien veut of si nous sumumes en vip. unus recoumeneroms. Vous ne puuvez vous di-prenser durénavant de chasser lautruche avec nous: row bledevenus de vrais Mekhalifs, aussi apples qu'eus at la ve du désert. $\infty$

Ces toraves gens avaient l'intention de nous faire un compliment, de plus neus étions de leur avis: ausi leur repondis-je avee convidion : " I lan prochain:

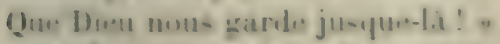

Iis-il heswin dajouler que liannee slivante nous

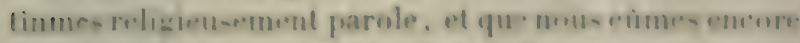

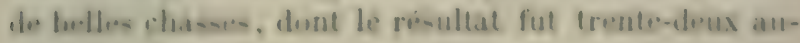
truches forcées.

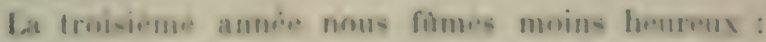

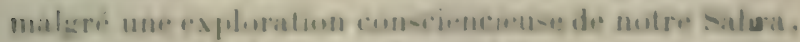
nums ne primes que douze aul rushes.

M. Phuletwert, qui asail ele nomme chef du bueau aralue de Miliana, nien sint fras moins chasser thus les ans avec nous.

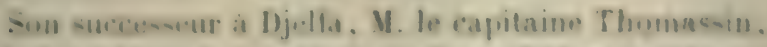
fut des nitres a la saison de la troincibue aunese Grand amateur de chases el do chevauchades, il compléta le trio.

Ce fut ares lui que mous accomplimes une course de suidante-dotaze lieues, qui ne fut usterrompque qque par 
une nuit de bal que nous vinmes passer à Laghouat. Jamais je n'ai vu de joie parcille à celle qu'éprouva le capitaine Thomassin quand il prit sa première antruhe : cela tenait du délire et se traduisait par des danses et des chants de la plus haute fantaisie. Comme il nous paraissait vrai alors ce proverbe des Mekhalifs : "Chasser l'autruche rajeunit! ” surtout quand on la prend, cela va sans dire.

Il avait été convenu qüil serait fait un tableau commémoratif de ce joyeux événement, avec notes, commentaires et musique d'une danse intitưée le Pas de l'autruche.

Je ne sais si ce tabléau a jamais été mis sur le chevalet; mais ce dont je ne doute pas, c'est qu il existe dans la mémoire de notre ami Thomassin, arec les personnages, le mouvement et la couleur qu'il avait à l'époque de sa conception.

A cette troisieme saison essistirent aussi quelques chefs indigenes de ma connaissance, de la subdivision de Miliana.

Un entre autres, Si-el-Habid, agha des Braz, vigoureux chasseur s'il en fut, était habitué à forcer le sanylier dans les courts espaces du Tell.

Il s'itait imaginé, malgré nos conseils, qu'il fallait lancer son cheval à fond de train et lui donner de l'éperon sans trève ni répit pour prendre les autruches de haute lutte.

Si-el-Habib arait amené trois bons cheraux pour pouvoir courir à souhait; au troisième courre, il ne lui en restait plus: il les avait mis sur le flanc par suite de boiterie, d'effort de tendons et de fourbures.

Son serviteur, un de ces vieux domestiques affectionnés, à la parole libre, comme il en reste encore 


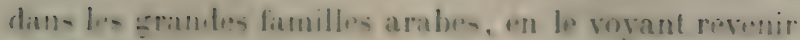

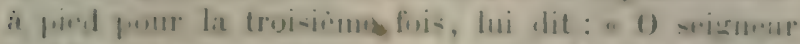
Habit, tu as encore ereve ion dernier cheral: Lih bien.

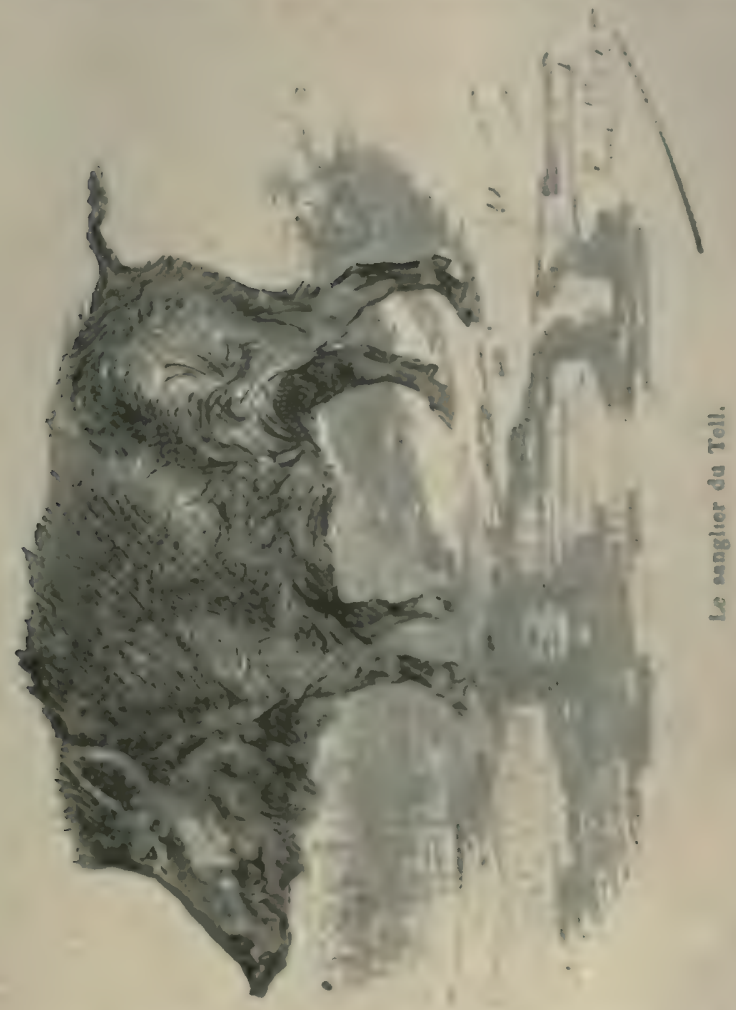

monle sur un chamean maintenant; c.ent ainsi que jo te ramènerai a tos fommes! w

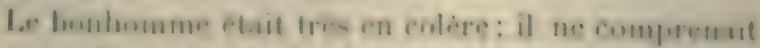
pas yow lion prit antsi, de gavele de curur, sacrili.r 
trois chevaux de prix pour courir après de grosses volailles, comme il appelait les autruches.

Tout en regrettant la mésarenture de son maitrc, nous ne nous attristions pas trop de ses jérémiades, nous comprenions, pour l'avoir éprouvée, la passion que Si-el-llabib avait pu mettre, comme hardi mais peu prudent cavalier, à courir ces trop intéressantes autruches.

Voir Naples et mourir! disait-on autrefois. Ge dieton est bien vieux et a fait son temps. - J'en propose un pour notre époque, avec une variante de circonstance : chasser l'autruche et... vivre lonetempls... pour recommencer!!! Pourquoi mourir, quand on a mieux à faire?...

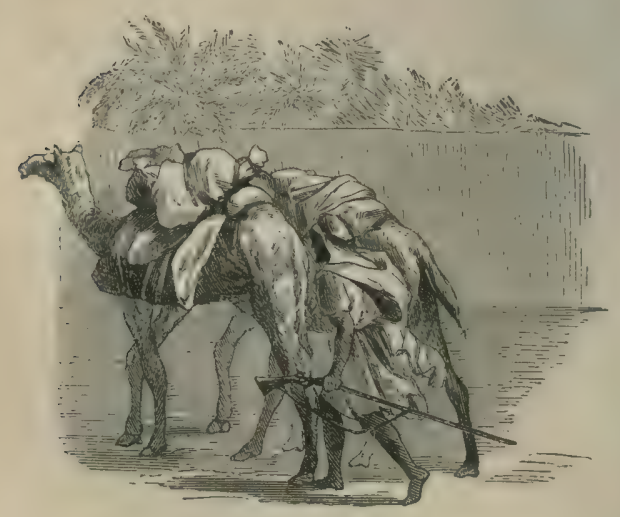




\section{L'AUTRUCHE}

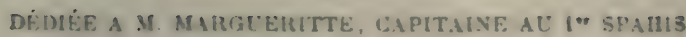
COMXANDAXT BUPERIEU DE LAG UOUAT

PAB ML. A. DB COUAVAL (1)
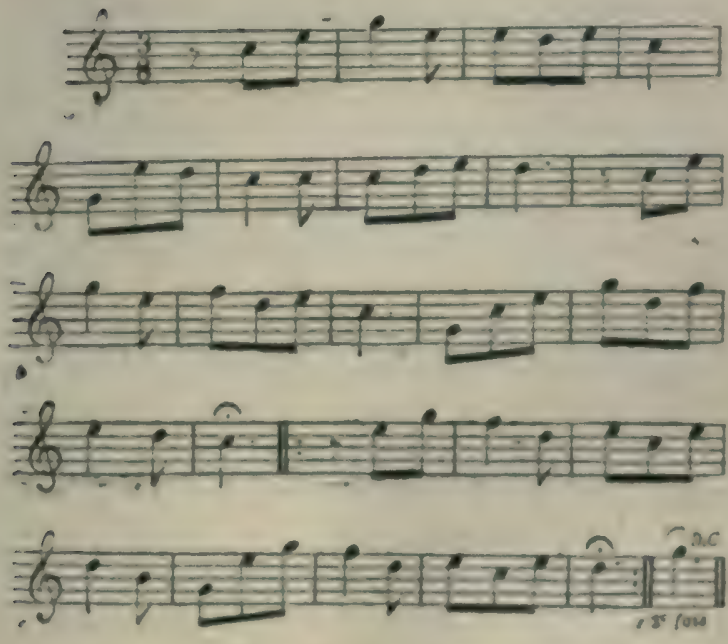

Laghtionat, le 24 juin 1855 .

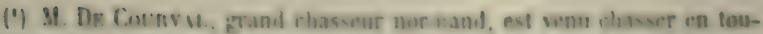

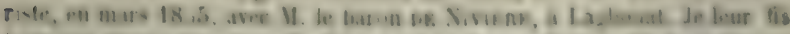

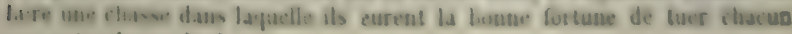
une autruclie au fusid. 


\title{
L E L I $0 \mathrm{~N}$
}

\section{DE LA FORÊT DES GÈDRES}

\author{
RENCONTRE FORTUITE
}

Dans thiver de 18:i, un lion vint prendre possession de la partie sud de la forèt des cèdres de Téniet-el-Hid. De là il raynunait à quelques kilomètres à la ronde et se sustentait aux dépens des donars de la tribu des Beni-Hayâne.

Je fus prevenu, dans les premiers jour: de janvier, quïl sacharnait particulièrement sur les bestiaux du Vieux de la Montagne, - El-Arbi-el-Hayàni, - personnage dont je reparlerai au chapitre suivant.

Je résolus d'aller passer deux ou trois nuits à l'affùt de ce lion.

J'arrivai donc un après-midi chez El-Arbi, qui me fit un bon accueil et me raconta, tout en aidant à mon installation d'affût $\left({ }^{1}\right)$, que depuis huit jours le lion

(1) Je dira une fois pour toutes que ces préparatifs consistaient, quand j'étais pressé par le temps, à faire un trou de deux pieds de profondeur, autour duquel je plantais quelques branches d'arbre pour me dissimıler.

Lorsque j'en avais le loisir, je faisais un trou plus profond que je recou- 
navail pas mangue une senle nuit de venir robler au-

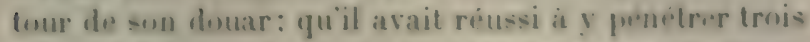
fuis et a lui prondre trois brebis: que, sans dunte, il roviembrait encore rette nuit, otc.

Jo dispusai mun affint a trente pas des fentes a un endruit yqu me semblait tres propice et par wis arrivait le lion.

Jy pharai comme appat un jeune taurillun malingre de peu de valeur.

Quand te suleoil se fut cuuches, je m installai dans mon tron, je veillat toute la nuil, mais vainement.

Le liun. contre liatlente générales, ne vint pas.

1. lendemain, je me remis a mon aftiot, espirant mieux. mais finalement sans autre resullat: jasais serulement constate que, vers minuit. les chiens diun dinar situo a emviron deux kilomotres de celui oi jo-

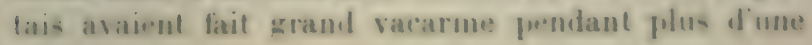
lieure.

Uuelifue chose: d'insolite arait dì se passor de ces cote.

Xious apprimes effectivement, dans la matumee, que le lion avait che prendre une brehis dans ce douar, et quiaprès favoir mangêe il avait do se réfugier daus la foret.

Peur celle fois, il n'y asait rien a faire. cinait partie romise. - Le temps, du reste, stait devenu mesuacant, Thurizon s'etait teint en moir et domongait ane grosse

vrais alurs de branclues solutes que comshtuanent un siritable alori cuntre te

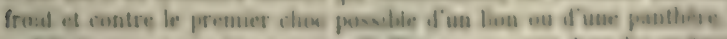

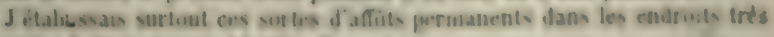

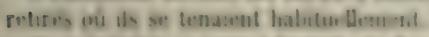

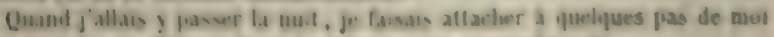

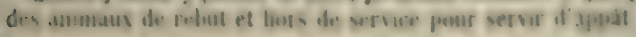


neige; le mieux était de regagner Téniet-el-Hâd au plus tot $\left({ }^{1}\right)$.

Il me fallait, pour cela, traverser une partie de la pente sud de la forèt des cèdres.

Lorsque j'y arrivai, suivi d'un cavalier qui m'arait accompagné pour prendre soin de mon cheral, je trouvai, au gué d'un ruisseau appelé l'oued Fersionan, un nommé Si-Yahia, chasseur de profession, arec lequel jarais chassé quelquefois le sanglier. Il semblait regarder avec attention quelque chose à ses pieds.

Après nous être salués, il me dit : "Voici les traces fraiches d'un lion; elles sont fortement empreintes dans la terre glaise des bords du ruisseau. "

Je mis pied à terre et je vis aussi ces grandes traces qui ne dataient que de quelques heures à peine... D après leur direction, c'était sans nul doute celles du lion que j'avais roulu affûter et qui arait été manger une brebis ailleurs.

Les traces allaient au nord vers Kef-el-Siga, partie très boisée et rocheuse de la forêt, où le lion avait probablement son repaire.

J'en fis la remarque à Si-Yahia et lui demandai son avis.

"Le lion, me dit-il, doit certainement se trouver dans ce bois touffu de chènes blancs que tu rois làhaut. - Il y a quelques jours, en poursuivant des gazelles ledmi $\left({ }^{2}\right)$, j'y ai trouvé de ses laissées toutes frai-

(1) Ténet-el-Hàd est situé à 1160 mètres au-dessus du niveau de la mer, les livers y sont très rigoureux; j'y ai vu la neige à demeure pendant des périodes de trente à quarante jours.

Le point le plus élevé de la forêt des cèdres, au pied de laquelle est Téniet-el-Hâd, est de 1986 mètres.

() Ledmi, grosse gazelle de nontagne ả longues cornes, double en grosseur de la petite et gracieuse gazelle scini des plateaux. 


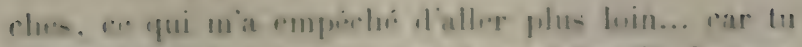

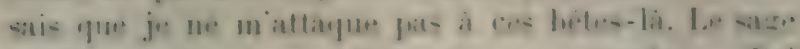

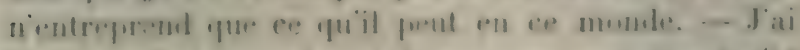

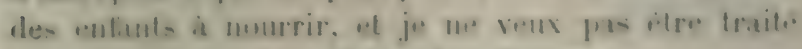

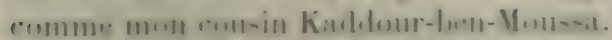

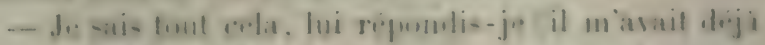

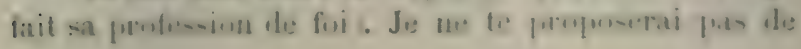

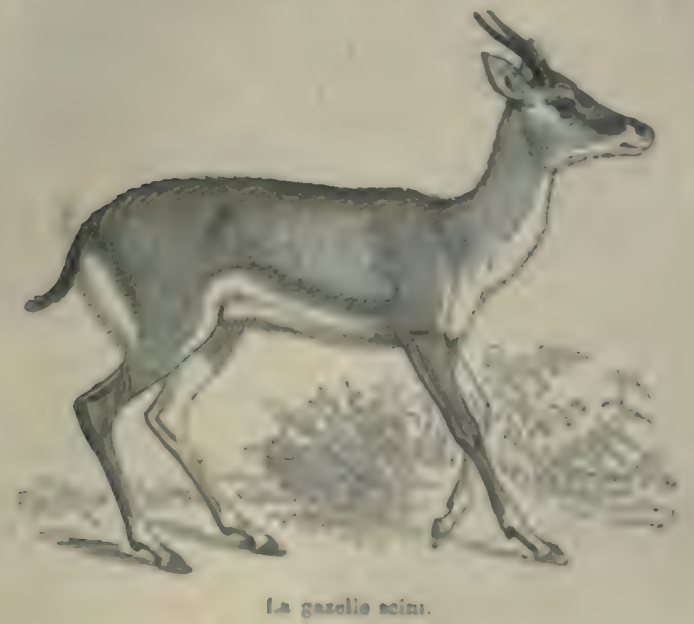

senir allatquer be lim dans son repmire: aldige-mon

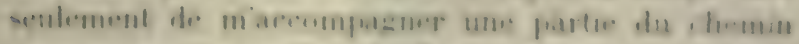

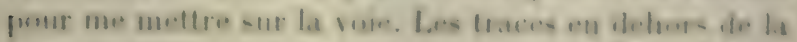

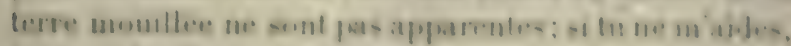

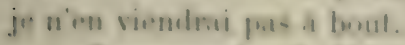

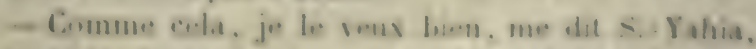

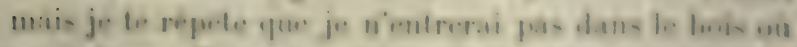

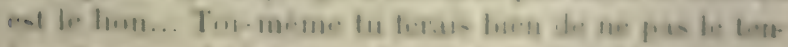

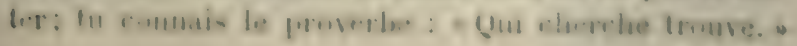


I. lion n'est pas une gazelle, et puis voici bientôt la neige qui va tomber; nous ferions bien de rentrer chacun chez nous.

- Fais-le si tu veux, répondis-je; mais puisque tu crois le lion là-haut, je veux y aller voir. Je viens de passer deux nuits à l'attendre, c'est beaucoup trop de peine pour rien. "

Et je me mis en marche, en recommandant au cavalier qui tenait mon cheral de me suivre de loin et de s'arriter quand il trouverait le chemin trop difficile.

Mon but était de gagner le bois en question, lequel se trouvait à environ quinze cents mètres de l'endroit où nous nous étions arrètés, d'y pénétrer avec précaution, de tàcher de tirer le lion si je le voyais; dans le cas contraire, de revenir prendre mon cheval et gagner Téniet-el-Hâd.

Si-Yahia, en me voyant partir, eut un moment d'inlécision; puis, par un bon mourement, il vint me rejoindre et se mit à me guider en marchant derant moi. De temps à autre nous trouvions, à défaut de traces, des foulées qui nous indiquaient que nous étions sur la piste.

Nous avions parcouru les deux tiers de la distance qui nous séparait du bois, sans trop de précautions, mais nous en primes davantage pour le trajet qui restait à faire.

Nous marchions doucement, nos fusils armés et l'œil en éveil. La neige commençait à tomber. Nous avions déjà dépassé plusieurs taillis, quelques autres restaient encore à traverser avant d'arriver au bois. lorsquen pénétrant dans un de ceux-ci, je ris Si-Yahia, qui marchait à deux pas devant moi et un peu à droite, s'arrèter net, comme pétrifié... Je m’arrètai aussi et 


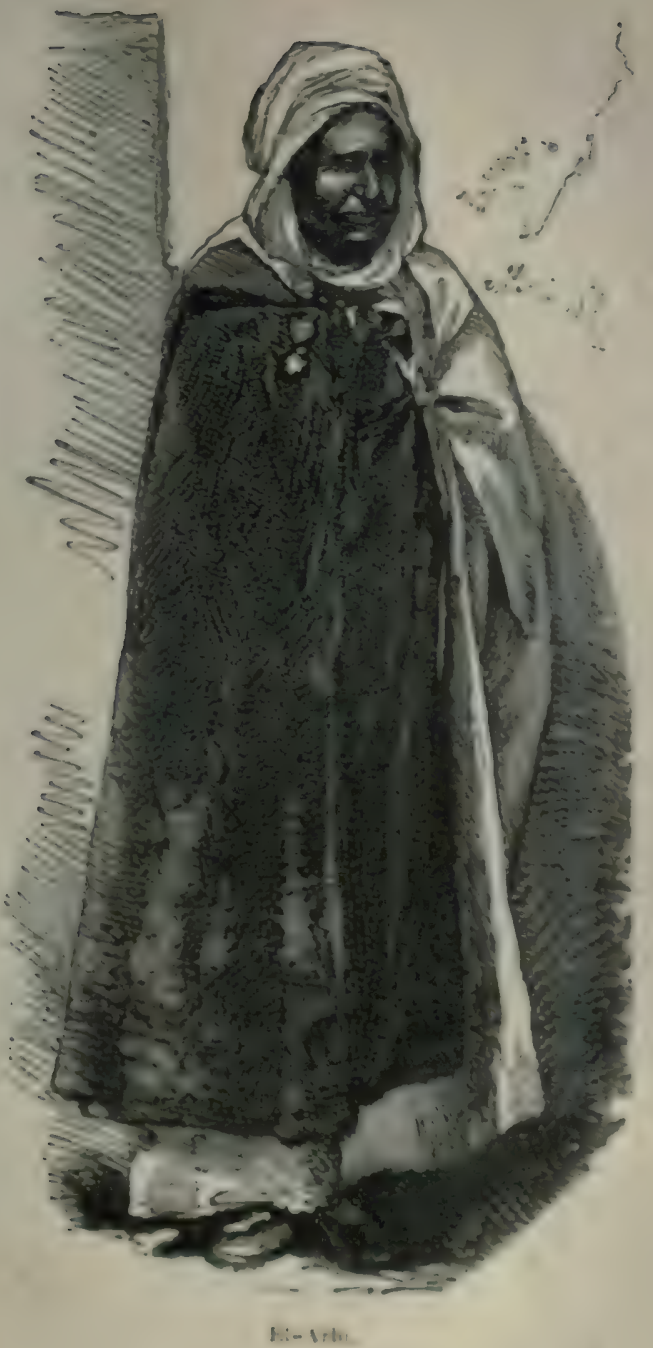





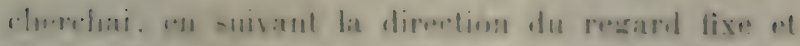

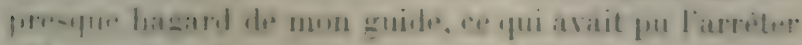

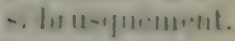

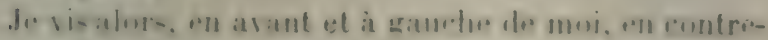

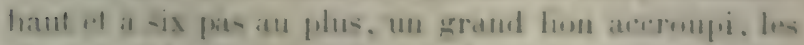

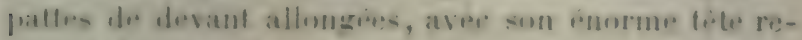

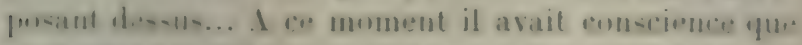

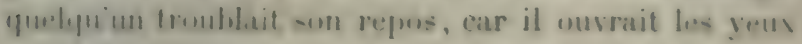
et levait la tele vers nous.

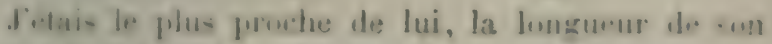

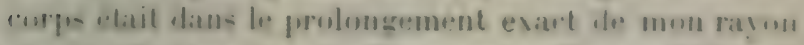

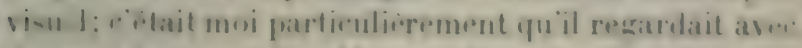
de stanuls youx limpieles et etomum, mais sans la moindre trace d'effroi.

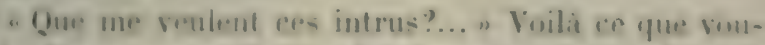

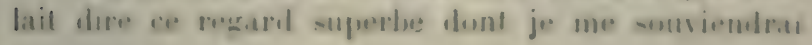
Loujours.

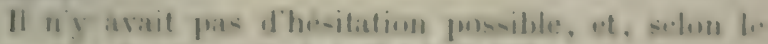
freosmber In bin etait tire. il fallat te bure...

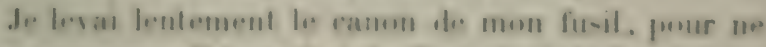

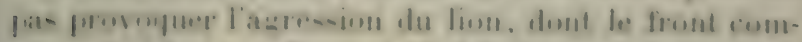

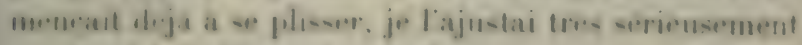

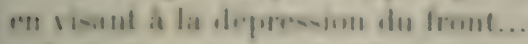

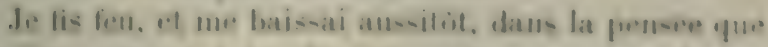

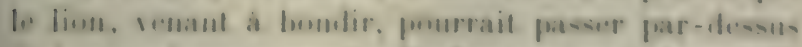
mon, e. qui me germellrall alopse ent me relournant.

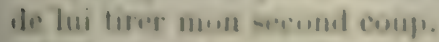

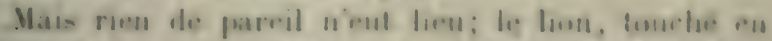

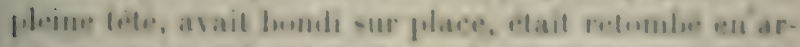

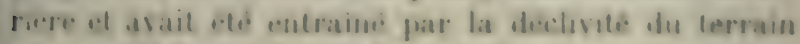

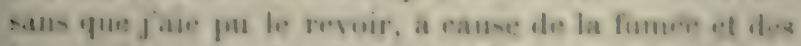

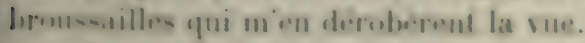

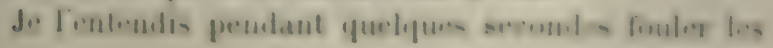


buissons et gronder sourdement en suivant la pente de la montagne.

J'avisai ensuite Si-Yahia, qui s'était tapi contre une souche d'arbre.

Il avait laissé tomber son fusil, qui s'était déchargé je ne sais comment; Jui-mème ne put me le dire. Il avait sans doute tiré en même temps que moi, mais sans viser et par un mourement machinal, car je retrouvai, en cherchant, sa balle enfouie à terre à deux pas de l'endroit où il s'était arrèté.

Je rechargeai mon fusil et me mis sur les traces du lion, que je pouvais suivre cette fois au sang qu'il laissait sur le sol et aux branches yüil frôlait.

J'espérais à chaque instant le trouver mort ou expirant; mes deux balles mariées ne pouvaient que l'avoir frappé au front, c'est-à-dire mortellement.

Mais la neige augmentait d'intensité à chaque minute; au bout d'un quart d'heure j'étais dans une véritable tourmente, qui ne me permettait pas de voir à quinze pas, et qui couvrit bientôt lesol de façon à faire perdre toutes traces.

Si-Yahia marait rejoint. - Cette fois je suivis son conseil d'abandonner la poursuite du lion.

Ce fut avec beaucoup de peine que je pris ce parti, mais il y arait cas de force majeure.

Je regagnai mon cheval et je repris le chemin de Téniet-el-Hâd avec mon cavalier, derrière lequel je fis monter en croupe Si-Yahia, qui, bien hébergé à la måison des hôtes pendant trois jours, se remit de ces émotions en prenant force tasses de café.

Le mauvais temps dura une semaine, le sol fut couvert d'un pied de neige; je perdis ainsi tout espoir de reconquérir ma bête. 
Ce niest que vingt jumrs apriss qpue dus patres en trouvierent les restes a peine reconnaissables dans un gros buision d'ui sortaient des rharals et au-dessus duyuel planaient des corloaux et dro vantwur:

Cens patres y entroment par rurinsité, ils virmot la carcasse d'un lion, avec des vestiges de eriniere et do patfos. Leurs maitres, auxquels ils firent part de cefte decouverte, vimrent me presenir. L'histuire de ma rencontre flait connue, ot ils ne doutaient pas que cee ne: lint la lo bun que javais tiré.

II itait alle mourir un peu au-rlessous du gui nu nous avions vu ses traces.

de the rendis aussitit sur les lieux, el je con-tatai,

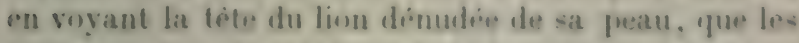
balles. an lieu de penelrer dans la cervelle. navaient fait que labuurer le erine dans sa lonemeur, assez foro. fombloment manmoins pour que mort sen-uisit.

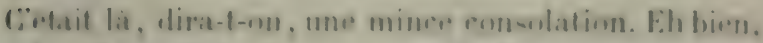

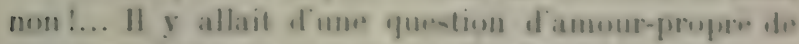

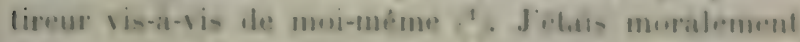

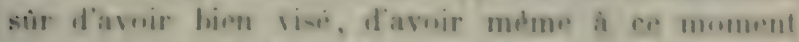

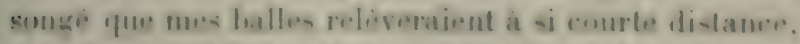

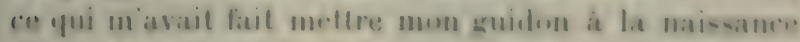
dufrout au lien de le mettre sur lo fromt méme de la hin.to.

Je voulais une rertifule matirielle, je lieus en tou-

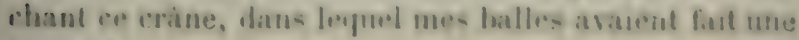
tranchion.

Bunreson, mon ami, - comme dimit Tepplter. -

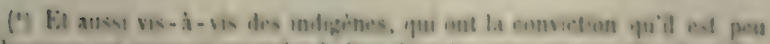

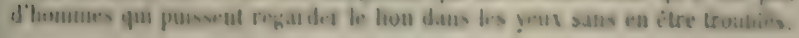


vous ètes pour quelque chose dans la recherche et dans la mise en évidence de ces preures! Hélas! nui, il y a 'juelque chose comme cela, et mieux vaut de suite en convenir... (Jui n'a sa petite pointe d'amour-propre en ce monde?

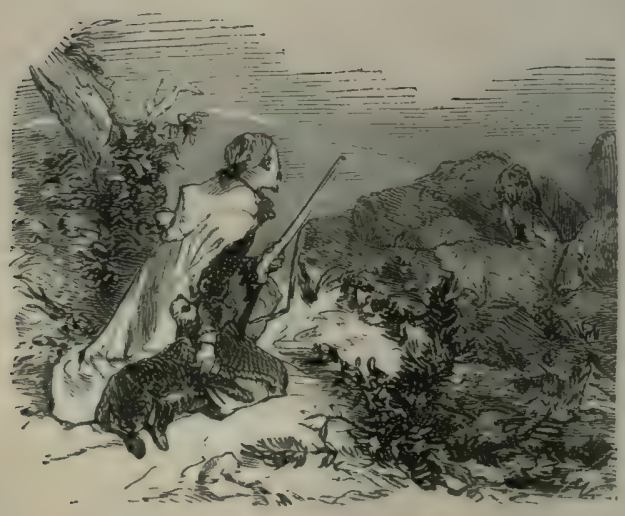


Il s'était trouvé à toutes les affaires sérieuses qui eurent pour théâtre le massif montagneux du Ouarsenis et les hauts plateaux du Sersou.

Il connaissait nos généraux et était connu d'eux.

Il les appelait familièrement Bugeaud, Changarnier, Saint-Arnaud, etc.

Quand il racontait le rôle qu'il avait joué près d'eux dans les grandes occasions, il assumait de très bonne foi la responsabilité ou le mérite de certains actes et s'exprimait ainsi : "Bugeaud, le jour de sa bataille avec les Beni-Boukhennous et leurs alliés, allait faire - tel mourement, lorsque je lui dis : "Bugeaud, crois" moi, je connais ces chiens-là et toutes leurs ruses. "Voici ce qu'il faut faire... "Il accueillit ma parole... et nous avons gagné ! " Ou bien: "Voussavez, mes enfants, la bataille de l'Oued-Fodda! elle a été rude! Heureusement j'étais arec Changamier, je ne l'ai pas quitté; quatorze fois nous avons chargé ensemble, et à chaque fois Changarnier me disait : - "El-Arbi, les "Arabes fatmas!" - C'était vrai, nous les renversions comme des femmes! ma balle ne tombait pas à terre. - Et avec Saint-Arnaud donc! Yous rappelezvous la grande razia que je lui fis faire sur les BeniMaïda? - J'eus assez de peine, c’était la première fois que le Roumiallait si loin. Il y arait une longue marche. de nuit à faire, de mauvais chemins; mais je dis à Saint-Arnaud: "Écoute, je vais aller moi-mème à la " découverte; je guiderai ta cavalerie, mon fils con" duira ton infanterie, et nous prendrons cette tribu " qui ne veut pas se soumettre. "Quel succès nous avons eu là ! - Aussi Saint-Arnaud me donne toujours la main quand il me voil et me dit: "Bono Larbi!" "

En dehors de ce qui était guerre, combats, aven- 


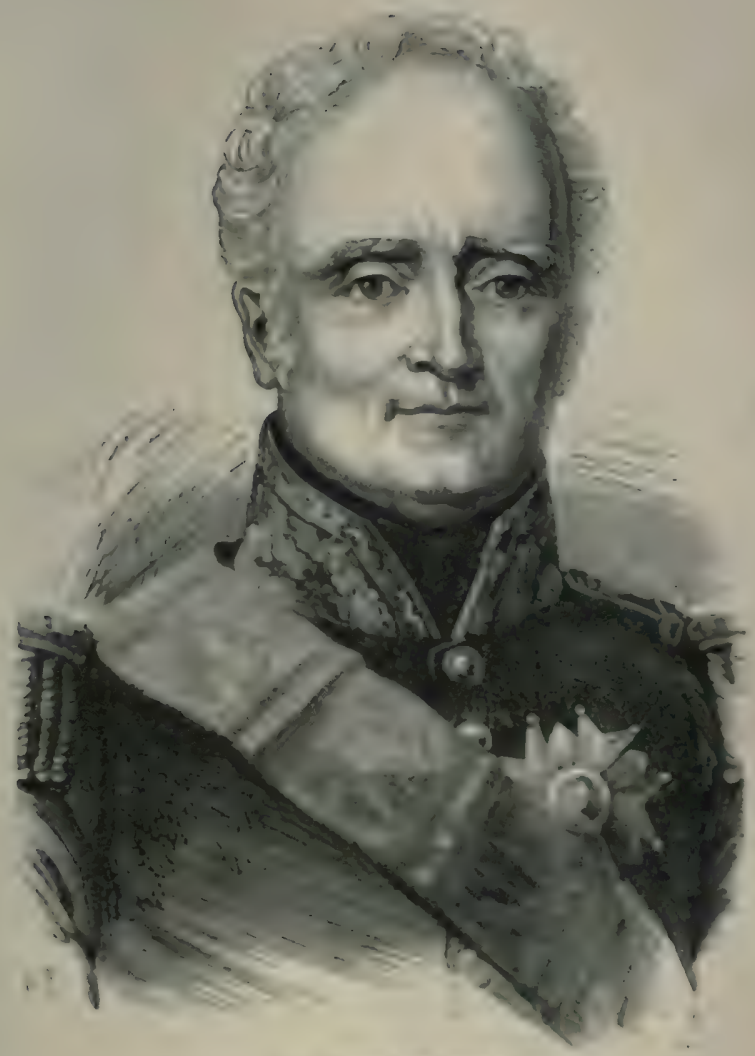

Le marectinel buspesual 



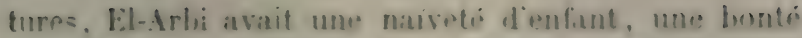

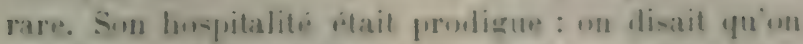
nes savat quil lemportatit chez lui de lhommen de poudre "uld de Flumme de tatum '.

Je liavis en affection puur sps bunnes qualitis ef sa franchise de combluite vis-at-vis de metre domination,

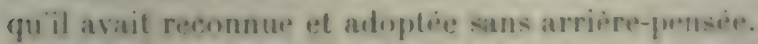

sumvent je prenais plaisir is lui faire raconter ses combatis aver les tribus hestiles. Bhal, Beni-Cithab.

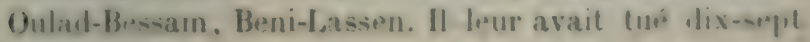
guerriers ?

En revanthe, il avait, fommesouvenir d'ulles, qualturze blessuress graves, dent lescicatrices lui couvanent le corps.

II ne lui restait que deux doigls mutiles de la main gaushe mais il trouvait encore moren de s.en servir

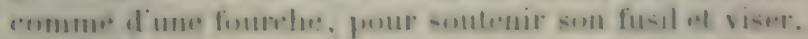

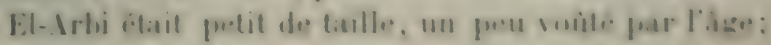

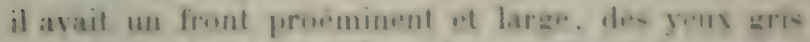

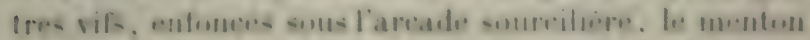

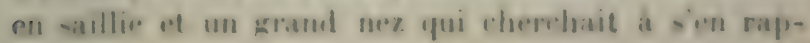
procher.

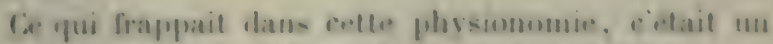

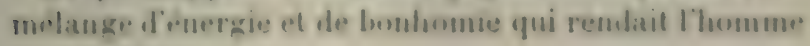
sympathique.

1.e Vieux deg In Montagne commenous le nommions,

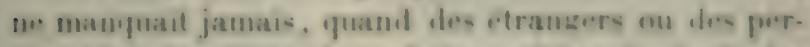

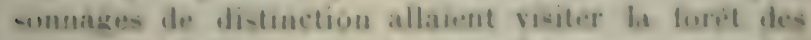

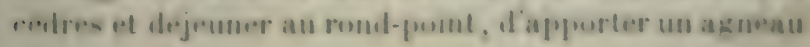

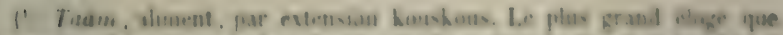

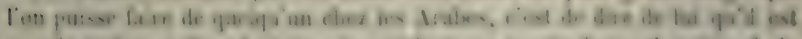

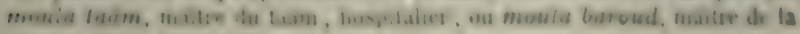

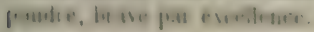


qu'il faisait rotir et qu'il offrait avec la meilleure bonne grâce.

Il était surtout ravi quand il y avait des dames. Il dépeçait alors lui-mème l'agneau avec ses doigts, selon la coutume arabe, et leur offrait les meilleurs morceaux, sans tropse préoccuper du peu d'orthodoxie du procédé... Il croyait au contraire se montrer très galant. C'est en adoucissant sa roix qu'il leur disait : "Mangez ceci, mes filles! le vieux El-Arbi a bien de la joie à vous recevoir. C'est peu de chose ce qu'il vous offre, mais c'est de bon cœur. "Et il $\mathrm{y}$ avait tant de simplicité dans ses manières, que les dames acceptaient gracieusement, et s'arrangeaient de façon à lui faire croire qu'elles mangeaient de grand cœur ce qu'il leur offrait.

Si j’ai parlé un peu longuement du vieux El-Arbi, c'est que je voulais le faire connaitre avant de raconter son aventure avec un lion.

Pendant les deux jours que j'étais resté chez lui, il Y avait eu de grandes causeries, des histoires du temps passé.

Il y avait toujours cercle autour du bonhomme, dont la mémoire était richement pourvue et qui contait bien.

Je savais son aventure, mais je voulais me la faire redire par lui.

A un moment donc où l'auditoire était nombreux et composé en partie d'hôtes étrangers à sa tribu, je lui dis : " O El-Arbi! comment se fait-il qu'un si vaillant . homme que toi, si maitre de son fusil, n'ait jamais chassé le lion ni cherché à se venger d'un animal qui lui enlève tous les jours des bœufs et des moutons?" 
Le brave humme une regarda d'un air étonné ot me dit: " 10 sidi Amarguerit, tu platointes, sans donute! Tu sai-bien yuil y a un pacte entre moi et les lions, yue je ne puis ni ue duis les combaltre, de quelyue façon que ce soit!...

b- Xim! fis-je, je ne savais pas cela, of lu me feras grand plai-ir de me le dire. Tes hoiles au-si en seront fien contents. " - Il ny eut quine veis pour laffirmative.

El-Irloi, evidemment ravi d'avoir une pareille oca-ion de placer son histuire favorite, se recourillit quelques instants, puis entama le recit que lion va lire.

- Il $y$ a hien des annees de celat mes eufants. citait du tomps du bey Muhammed-el-Kehir. Jretais joune alors. ce-t a peine si le proil fitait levé sur ma figure. -

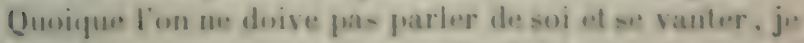
dois neannenins vens dire que jetass alore cite peour un Lun cavaluer. - Javais echange des bulles aver nus wor-

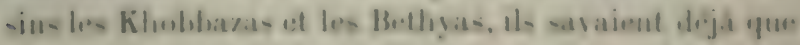

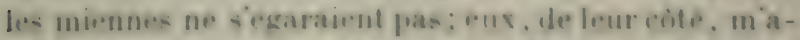
vaient troue la peau, - vogez crotte blewne a la jamber sauclue et celte autre an cont. - Javais recu la premiere a la jouruee des Silus-des-Amandurs, ot la orconde dans notre combere 'I sur les Lroupreaux dos beru-

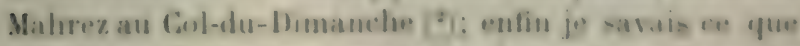

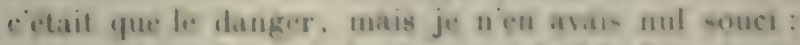
mes melleurs jours chaient ceus ou je daisais parles la poudre.

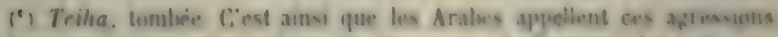

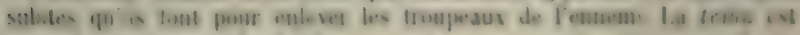

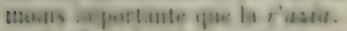

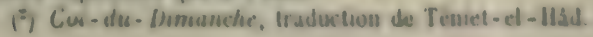


"Une: année, nous avions établi notre campement dhiver à la Colline-des-Gilands, sous Kef-el-Siga.

"Cétait bien près de la forèt et des repaires du hoûche (1); mais nous étions alors en guerre ouverte avec les Beni-Chaib et les gens du Ouarsenis, il n aurait pas été prudent de rester en plaine en butte à leurs altaques; mieux valait ètre exposés à perdre quelyue bétail par le fait des bètes, que d’ètre complètement raziés par nos ennemis.

" Ce que nous avions prévu toutefois arriva.

"Nous n’étions pas dans notre mérheta depuis deux semaines, que déjà nous avions eu trois bœufs de cassés et cing ou six brebis enlevées par le lion, du milieu de notre douar, malgré les grands abatis d'arbre: dont nous étions entourés.

"Mon père et mes oncles, que Dieu leur fasse miséricorde! étaient très peinés de ces pertes; de plus, le -ommeil arait quitté nos yeux; nous passions toutes nos nuits debout pour crier et éloigner l'ennemi, mais sans profit.

" Un matin, après aroir veillé, crié, lancé des tisons enflammés, et, malgré cela, avoir vu notre enceinte franchie, une nouvelle brebis enlerée, je sentis le sang bouillir en moi, et je me dis dans mon àme que nous ne pouvions vivre ainsi.

"J'excitai mon père, mes oncles et mes cousins.

(') Houche, nom donné au lion par les Arales de cette contrée; il vient de haïcha, bête. Ils le désignent encore sous le nom de metelouf', ėgaré. J'ai remarqué que les Arabes qui avaient à redouter l'agression du lion ne l'appelaient jamais de son trai nem, Sluâ.

Ils ont la croyance que celui-ci comprend son nom de Sbà et répond par sa présence, toujours fàcheuse, à ceux qui l'évoquent de cette manière.

Nolre proverbe populaire: "Quand on parle du loup, on en voit la queue ", a quelque analogie avec cette croyance. 


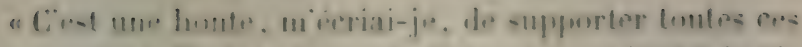

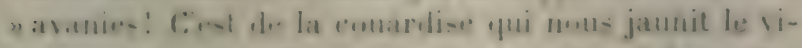

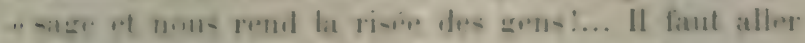

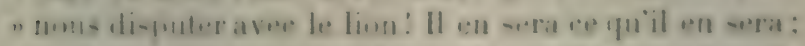

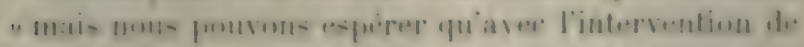

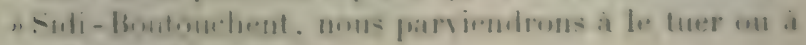
- Delingued de nums,

a fous de la peine dabord a derider mon prive et mes uncles: - ils nasateut jamais wulu saltapluer an lions, dans la rrante de Saftirer teur mimilie. - Maique funsait-elle de plus? Jes ne l'imaginatis pass encome.

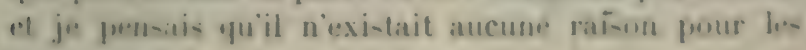
épargner à l'avenir.

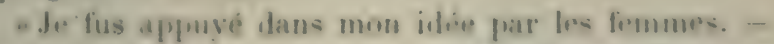

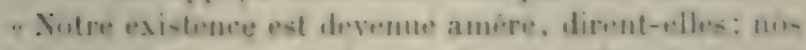

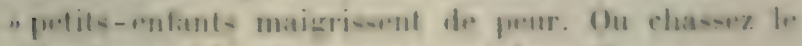

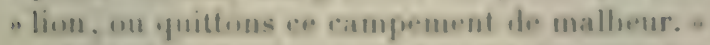

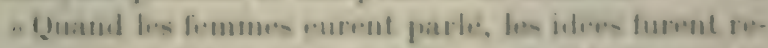

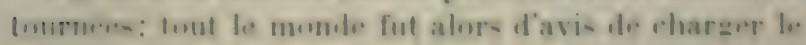
lion.

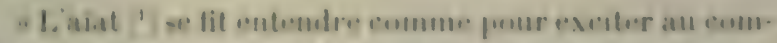
bat. L.c- hommes se coignirent of prirent leurs armes II news en sut des denars bui-ins. qui etatent de mo.

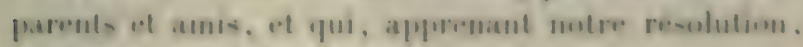
voulurent an joindre a nem-.

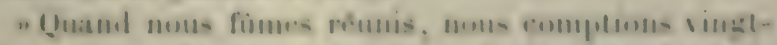
sis homumes, fous aree des firsils. lees frommes les pluse

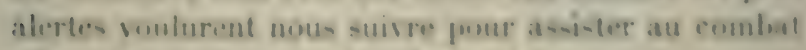
e.t mous entourager an besin.

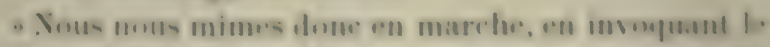
maralusut sidi-Bbutum-hent: - pust mon comple, je

(9) Jor un dis chapitres a - apres. 
lui vouai une ouâda (1) de mon plus beau bélier et de six djefnas de kouskous, si nous réussissions.

.. I. lion qui avait mangé nos bestiaux dormait dans le fourré des Fernanes $\left({ }^{2}\right)$, - leur repaire de prédilection quand ils viennent dans le Kef-el-Siga.

"C'est là quil fallait aller le trouver.

"Notre plan était de nous mettre sur deux rangs, d'approcher à vingt pas du fourré, après avoir préalablement laissé les femmes sur un rocher en arriere, et de défier le lion pour le firiresortir; une fois en vue, de faire sur lui une décharge générale qui ne pouvait manquer de le tuer raide.

"Tout cela bien convenu, nous approchimes du dortoir du lion, excités par les tzagr itz $\left.{ }^{3}\right)$ de nos femmes.

"Au premier rang étaient les hommes les plus valides et les meilleurs tireurs. On s'arrêta comıne il a été dit; les fusils furent armés et la crosse mise à l'épaule.

"J'appelai alors le lion et lui dis : "O mangeur de "boufs, sors de ton repaire! Viens voir en face des " hommes! C'est aujourd'hui le jour du payement!" II né répondit pas.

"Vous savez, mes seigneurs, qu il en est quelquefois ainsi, et qu'il faut répéter l'invitation pour faire sortir le lion. Je la répétai donc en ajoutant: "Ne fais pas le

(') Ouâda, veu. - Les Arabes en font souvent à leurs marahouts de prédilection, quand ils entreprennent des aventures difliciles ou quand ils veulent la réalisation de choses qu'ils ont grandement à coeur.

Le mouton inmolé pour la ouada et les plats de kouskous qui l'acconpagnent sont mangés par les indigents, qui sont prévenus à l'avance du jour et du lieu où elle sera servie. - La djefna peut rassasier dix hommes.

(i) Fernanès, cliênes-lières.

(3) Tzagr'it $\%$, - sorte de cri aigu et modulé, prolonge jusqu’à ce que la voix vieune à manquer. - Ces cris excitatils sont poussés par les femmes arabes dans les fêtes et dans les combats, ou bien encore devant un grand personnage, pour lui faire honneur. 
des pierres dans l'entroit où nous pensions qu'il étrit".

"Uh! alors, mes enfants (et en disant cette phrasr. Vil-Arbi oscillait sa tète de droite à gauche , si rous avicz ru cela.... Le tonnerre se mit à parler par li bouche de ce lion, et comme un éclair il tomba devant nous.

"Nus fusils partirent, mais il n'eut pas l'air de s'en apercevoir. Il s'élança sur le groupe du milieu, qüil prit dans ses pattes, et mit trois des nòtres sous lui. mon cousin Ben-Meftah arec la tète fracassée, le fils de Ben-Smail avec la poitrine ourerte, et mon oncl. Rabah, qui, par la protection du Prophète, n'avait pade blessures graves, mais qui, se voyant sous le lion. nous criait : " 0 mes frères, délivrez-moi! Par la figur"s "de Dieu le Très-llaut, sauvez-moi de ce péril! "

"Presque tuut le monde avait fui en royant ce que le lion avait fait des hommes; mais les femmes nous firent honte, surtout celles qui avaient un parent parmi les trois qu'il arait couchés sous lui. Ma cousine Aïha. qui devait être ma femme, pleurait et s'arrnchait les cheveux en voyant son père Rabah dans cette position. lille me criait: « El-Arbi, délivre-le! délivre-le, ou jamais "je ne te regarderai! - Je suis à toi! " m’écriai-je: et je m'avançai sur le lion pour le brûler avec mon fusil, ne voulant pas le tirer de trop loin, dans la crainte dr. blesser les hommes qu'il tenait. Il me laissa approcher d' trois pas; mais au moment où je l'ajustais à la tète, il se redressa, et, d'un coup de patte, m'arracha mun fusil, dont il fit une faucille $\left({ }^{1}\right)$. He trcurant ainsi desarmé, je me reculai d'un saut en arrière et me mis it fuir; mais l'affreux hoûche était sur mes pas... Je sentis

(1) Qưil tordit en forme de faucille. 


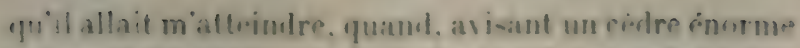
you avat ele abattu ol gisalt sur lo sul, je me jetai dos-

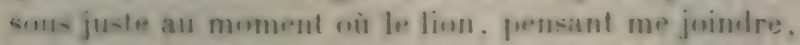
alail leve ses deux patles pour mesai-ir. Motant brus-

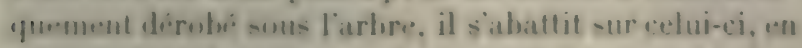

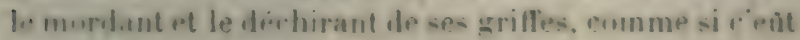
\&té moi.

- Yous voyez ma pusition. mos enfant-.... elle navat

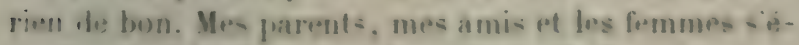
Eratiznaient les jours ell sigue de oleuil. On me erovait arharpes: jentemdais les lamentations que lon faisait - 115 inun sort.

- Jendant ce tempos. In liun etrit "ul travers de larbre et und desisnus. Ses dexux fattes de devant premlaient dincité, celles de derriere touchajent lerre de Tautre. II surtait de sa guexule des grondemesuts elfruyables.

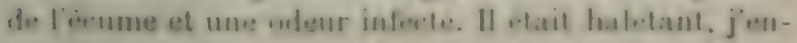
londal souffer sa poitrine comme - i rlle rit contenu la tempete.

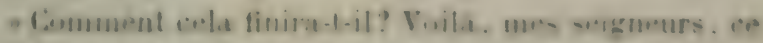
que je" peensais. II n y abal pas a compler =ur le secour-

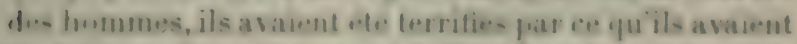

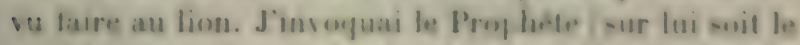

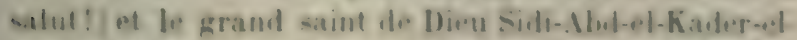
Djolam \%). Ils euremt pitio de mosi... Cine inspumation mo. vant... Javais entemulu dire que le lion comprenait

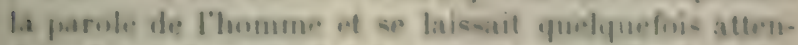
drir. Je madromal a lui d. celle faron : = 1) sullan doe

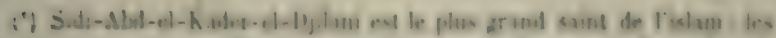

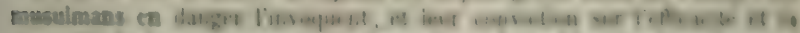

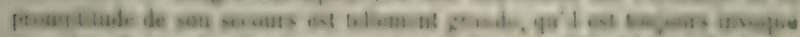

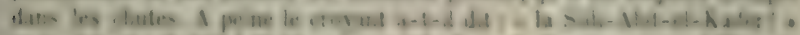

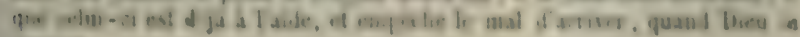
fin: $: \cdots 1$ 
" animaux! tu es le plus fort. sois généreux contre ton " ennemi vaincu. Si tu me laisses la vie, je prends Dieu "à témoin que jamais plus je ne m'attaquerai à toi, ni ") à ceux de ta race."

"Le lion, comme s'il m'eût compris et accepté le pacte, rugit encore une fois, puis quitta sa position de dessus l'arbre, et se retira lentement vers la forêt, en jetant de temps à autre un regard de mon côté.

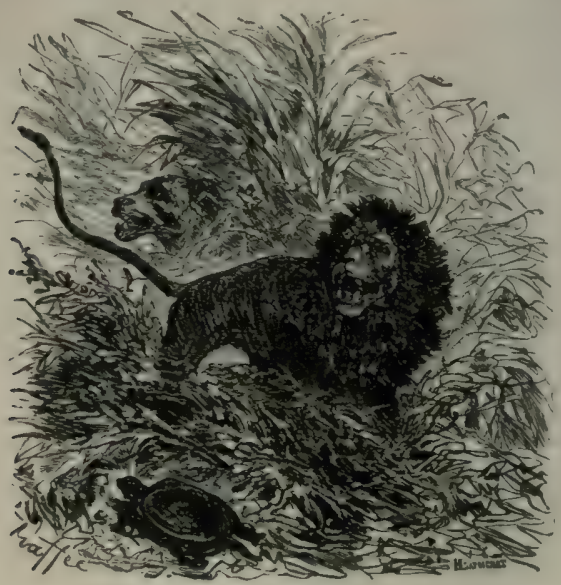

Le lion.

"J'étais bien joyeux, comme vous pensez, de voir le lion s'éloigner, mais je n'osais sortir de dessous mon arbre pendant qu'il était en vue.

"Ce n'est que lorsqu'il fut rentré dans le bois et que je l'entendis dire par mes compagnons, que je me relevai et'me mis à courir vers eux, comme si j'avais eu des ailes. "Je fus accueilli par tous avec des cris d'étonnement et de joie. 


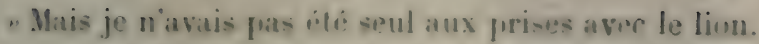
de propmai dialler relever les truis humm. s quil avait I'abord abaltus. Quel spuretacte, is envoyé de Dieu .... Ben-Meftah rtait mort, ... sat tite chait en fromage $\%$ : - Ben-Smal sivalit enrore. mats il avait la fmilrine unverte et devait mourir dans la journee: - enfin, mon oncle navait que des contu-ions, mais il avait ete foule par la poitrine du lion et se trouvait évanoui.

".ous dumes les rappurler tous les trois a mos tentes. vi les lamentations du deuil durierent huit jours.

n Vuilà, mes enfants. ce qqui est arrivé de moni aree le lion.

- J'ai eu bien de la peine davoir éte cause de la mort de deux hommes : aussi, depuis ce jour. jai temu parole... el jamais, quolqüil mait mange hien des lopuls et des brebis, je n ai sunge a me ballre de nouveau avec lui. Cobitit convenu, - on ne doit asnir qu'une seule parole.

- Je sais Lien, ajunta EI-Irbi en mannire de perm. raison, quil y a des formmes yui tuent le lonn conme si cer netait qu un chien, wais cint par la peermim-ion

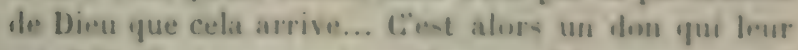
at liat, ils ne provent en tirer vanito. parce que st

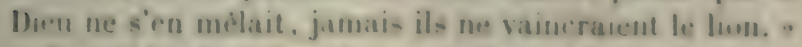

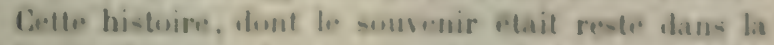
menure des gons du pass, nous asat fortement inte. remens.

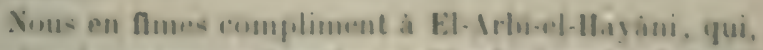

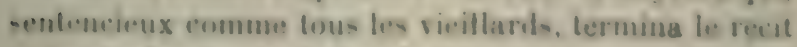
- da reunion par cetle masime:

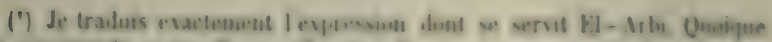

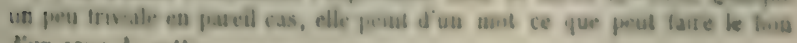
d'un coup de patte. 
"Il n'y a, mes enfants, de force et de puissance qu'avec l'aide de Dieu : tout passe en ce monde, lui seul est éternel!

* Allez avec le salut!

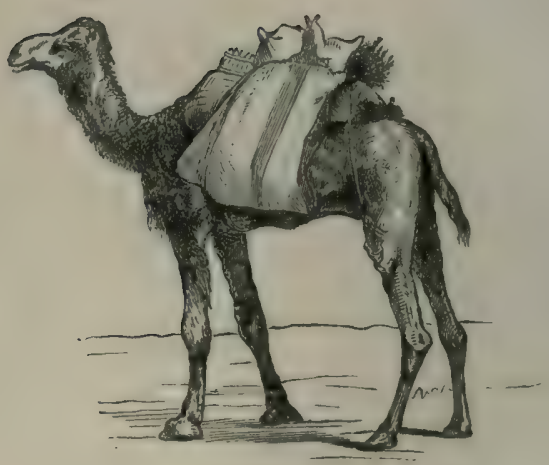




\section{A \\ CHASSE AU FAUCON}

La chas-e au lausun, en .lgerie. est restie lapanage dies grandes findille- du pays.

lie- un des principans reliefo de la veritable ari-lormalie araber.

Quelefues fartenu- ont escaye de se le demnes; mans

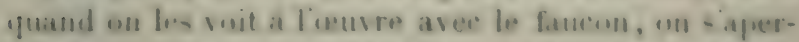

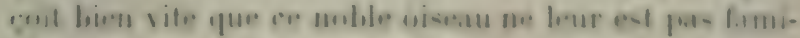
lier, et quil est deplace cutre leurs maine.

Cert flien ellet on ule simprowion pas malle en

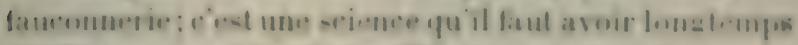
- dublice ou peraseder de tradition.

Las grandes familles de la proviuce d'Alger qui se

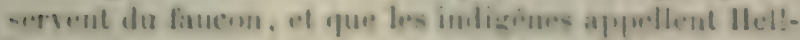

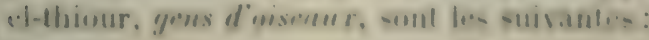

J.es Oulat- Vuldatar:

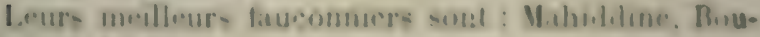
Disa, Lahlyilar.

les Oulad-Chand:

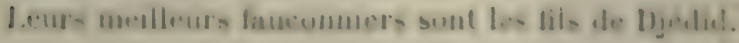


Les Oulad-Nayls :

Leurs meilleurs fauconniers sont : Kouider-benLegbèche, Telli-ben-Lekhal.

\section{Les Bou-Aiche:}

Leurs meilleurs fauconniers sont: Slimen, Abd-elSelam, Rahmoun.

Les Oulad-Aïssa :

Leurs meilleurs fauconniers sont : Si-Djelloul, Siben-Salem.

Les membres de ces familles djouades ( $\left.{ }^{1}\right)$ chassent de père en fils; ils ont, pour les aider dans le service des faucons, des façons d'écurers-fauconniers, plus particulièrement chargés de prendre les oiseaux de race, de faire leur éducation, de les nourrir, de les porter et d'aider à leur rappel quand on vole le lièvre ou l'outarde.

Il y a parmi ces gens, que l'on appelle biázes, oiseleurs, des types d'une grande originalité; le fond do leur caractère est un amour-propre démesuré à l'elldroit de leur science en fauconnerie.

Il y a deux sortes de faucons:

Les étrangers,

Les indigènes.

Les premiers sont préférés, ils sont très courageux el chassent aussi facilement la plume que le poil. Les fauconniers du moyen âge les appelaient sors; ils viennent le plus sourent de la Suède, de la Norvège et de la Finlande.

Ce sont des faucons de haut vol, genre gerfaut, arec lesquels on attaquait, au moỳen âge, le héron, la grue, l'oie sauvage; etc.

(1) Djunades, noblesse militaire. 
Le faucon indigine de l'Mlgérie est celui disigni en hi-tuire naturelle sous le mom de lanier. II mol ans-i tris brave, ot de haut rol; l'élucation developple sess qualités naturelles.

Pour prendre los faurons, les biazzes se servent de forrolix, de pigens of de ganeas.

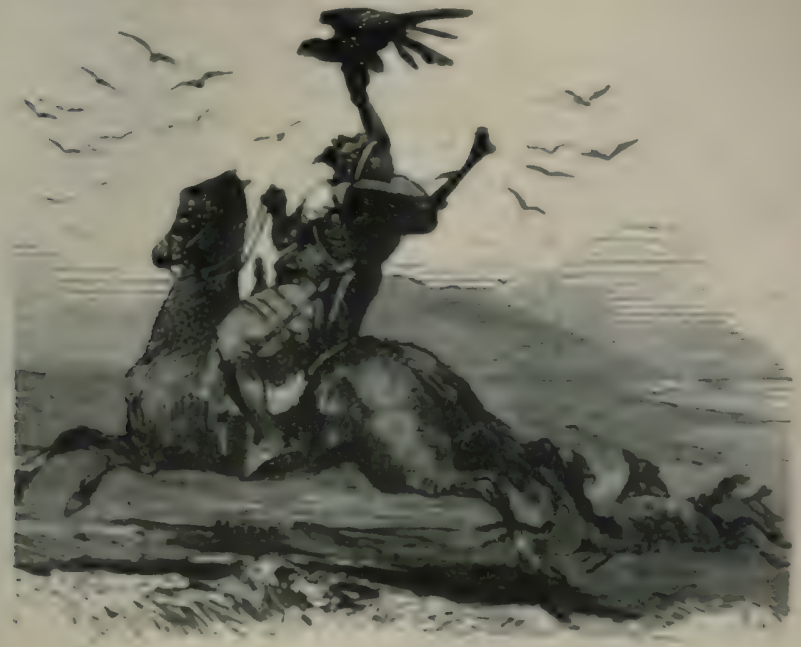

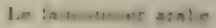

Ils enseluppent cos velatiles diun reseau de laes of

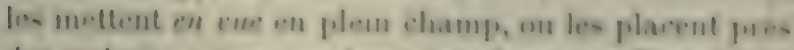

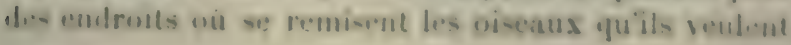
prendre.

Le faucon, ens se previpitant sur ce quil erout atre

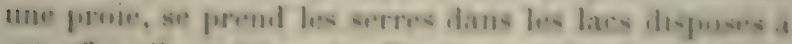

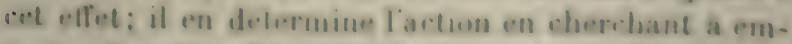
purter liappint yui est allache a une ficelle fivie a une piorre assez lourde prour ne pas étre enlevee. 
Le biâze, qui est resti i l’affut, s’approchr alurs avec précaution, s’empare du faucon, quil coilfe touí d'abord d'un chaperon pour lui ôter toute défense.

Il lui met ensuite de petites manchettes en cuir. auxquelles il attache des lanières de six à huit pieds de longueur, rattachées par leur autre extrémité au want en cuir à la crispin que porte tout fauconniur lorsyu'il a son oiseau sur le poing.

Le dressage de l'oiseau de race se fait à peu pris de la mème manière quil est indiqué dans les anciens "Déduicts de fuuconnerie " du roi Modus ou de Gaston Phobus.

Trente ou quarante jours suflisenl ordinairement jour amener le faucon ì fondre, an milieu des gons et des chevaux, sur les lièvres et les outardes, a les prendre à pleine serre, à les tuer à coups de bece, ì ubeir au cri de rappel, et enfin à venir se poser sur Je leurre quand la proie a été manquéc.

Chaque faucomnier élève plusieurs faucons, farmi lesquels il fait un clroix des meilleurs.

Pendant la période d'éducation des oisfaux, des renseignements sont pris par les fauconniers sur le nombre et le degré de perfection des faucons dr tel ou tel djouad. Une grande émulation, des rivalitŕs, s'emparent de leur esprit. Souvent ces rivalités s'utablissent entre les membres d'une mème famille, il en résulte des défis et des paris sur le plus de force ou de sagacité qui sera déployé par tel ou tel oiseau.

On donne aux faucons des nums qui sont zresque toujours ceux de leurs maitres, ou de personnages connus par leur braromre el leurs prouesses.

La saison de chasse une fois terminée, la liberté leur est rendue. 
On leur mel frialidilement, quand ils ont du mérite. un maryue à laquelle on jui-ser les reounnaitre plus tard: suit un ammau d'or ou d'argent antuur diune serre, aver un chiflie, wu des pointes de feu à la naissance du bec.

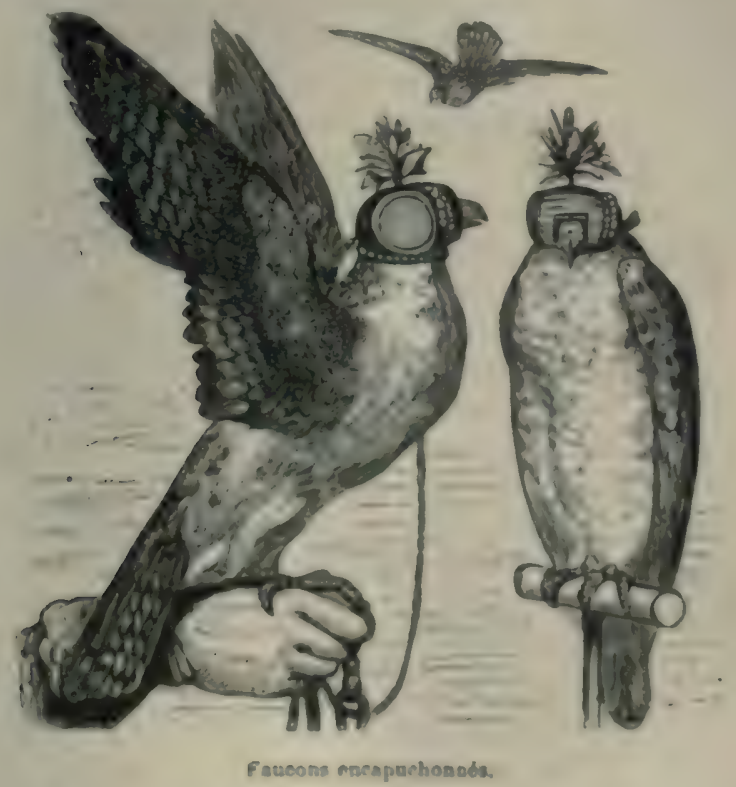

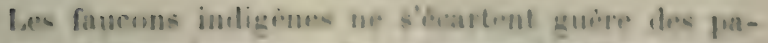
vages un ils naisent: on les refromse et on ies reppend

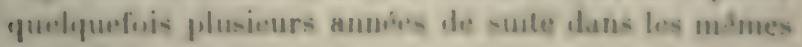
sendroith.

(linud un fancomnier capulure un ojecau de race mar. quil d'un sigur autre que le sen, il ent lemu de le rendre at son proprielaire. sil te commait. 
Quelquefois néanmoins, et par exception, des fanconniers gardent d'une année à l'autre des oiseaux tout à fait hors ligne, auxquels ils se sont attachés.

La chasse se fait de la fin de norembre à la fin de février.

Pendant ces trois mois d'hiver, l'oiseau de race a tout sa vigueur, ses plumes ont acquis tout leur développ"ment; son appétit, qui est considérable, le stimule encore.

Mais, vers le mois de mars, arrive la saison deamours, qui lui fait abandonner la chasse et son maitre, si, à ce moment, on ne lui rend sa liberté.

Quand les oiseaux de race sont drescés, et que le moment de s'en servir est venu, les djouades s'avancent, avec leurs familles, leurs clients, leurs sterviteurs et leurs troupeaux, vers le Sahra.

C'est alors une grande joie pour tous, car cette rígion possède un attrait puissiant qui agit sur toutes les organisations et sur tous les àges.

Vieillards, adultes, femmes, enfants, considèrent comme un jour de fète celui où ils quittent les pentes plurieuses du Tell pour s'enfoncer dans le pars du soleil.

Les animaux eux-mèmes sont accessibles aux charmes de cette transhumance hivernale, qui leur promet un climat plus doux et des pâturages plus précoces.

Quand donc les campements sont itablis en plein Sahra, que différentes régions ont été explorées, on commence la chasse, qui s'exerce uniquement sur les lièvres et les outardes.

On ne peut bien chasser au faucon que dans un par's découvert où le gibier, une fois lancé, peut presque toujours se voir, et où le faucon, en fondant sur sa proie, ne court aucun risque de se blesser. 
Los immenaes pleimes du Sud, couvertes done vegrefation dialfa et darmoise? qui ne forme pas dioh-tarles. sont essentiellement prepices pour le wol.

Ciet vers deux heures de l'apris-midi que se fait ordinairment le dopart do lat chasse, paree que la limim, qui est le principal stimulant des oispaux de tatre, ne sp prononce que vers ce moment, quand its ont eté repus la veille.

Les reunions pour le vol au fiucon se composent presque toujours :

Des djouades ayant leur faucon favori sur le poing;

Des hisizes aver trois ou quatre fucons quil portent. un sur le poing gauche, un sur la tóte et un sur chayue epaule;

Des parents, étrangersou invités:

Enlin, d'un plus ou moins grand nombre de cavaliers et ife serviteurs pour trajuer et porter le gibier.

Le depart est plein d'entrain. L'Arabe. Lujours grave dans tuus les artes de sa vie, laise vir dans ce moment-lia une partie de la passion qui l'entraine.

II est gai, il rit voluntiers, see gestes sunt animes. II fait caracoler son cheval devant les tentes do douar, oil il sait que des yeux le regardent avec des sentiment, yuis ne sunt pas ceux de l'indillerence; il parle à son faucon, lui demande s'il se compqortera dignemest, s'il yf a de lu', anjourd hui.

Enfin tous so mellent en marrh. en imsuguant le nom de Dieu.

Quand on est arrives sur le terrain ou l'un comple Irouver le gibier, on se forme sur whe ligne un preu concave, les fauconniors au centre. Tuus les assintants smit repartis aus ailes et dintancés entre eus de quelynes pas. 
Au signal du chef qui dirige la chasse, la traque eommence.

On marche au pas, on fait du bruit en frappant de l'éperon contre l'étrier et en criant de temps à autre i pleins poumons: haou! haou!

J.es traqueurs agitent les pans de leurs burnous comme suls chassaient vivement des mouches. Ces gestes effrayent et font lever les lièvres.

On dirige les chevaux sur les touffes les plus épaisses d'alfa ou de chihh; on fouille celles-ci avec de grands bàtons à l'extrémité desquels est une petite fourche. pour prendre les lières au gîte ou les relancer quanıl iis s'arrètent effrayés au milieu des traqueurs.

Enfin, chacun fait $c$ : qu'il peut pour faire Jever ces: pauvres lièvres, qu'une sorte d'instinct semble prévenir des dangers qu'ils vont courir, et qui ne débusquent qu’à leur corps défendant.

Les faucons sont débarrassés des lanières qui retiennent leurs manchettes en cuir.

Ils sont portés sur le poing à la hauteur de l'épaule.

On leur laisse la tète couverte du chaperon; il n'ost fait d'exception à cette règle que pour les vieux faucons, bien dressés, qui chassent de l'œil sur le proing de leur maitre.

Il y a avantage, dans ce cas, à déchaperonner l'oiseau, parce que sa vue est tellement perçante qu'il découvre, bien arant l'homme, le gibier levé. Il fait alor's des tentatives pour prendre son vol et attire l'attention du chasseur vers le point où il regarde.

Voilà à peu près tous les préliminaires du vol au faucon; je les ai amenés au point où l'action va commencer.

Comme j'ai beancoup pratiqué cette chasse, je vais 
pronire, dans mes suluvenir-, une de cellne qui onf sté houreuses, et en faire le récil. Elle donnera un. idese le cet exerice si particuliorement gat et plecin des mourement.

Pendant que jo commambais lo ererele de Laglumat, ju chassis au faucun toms les hiore.

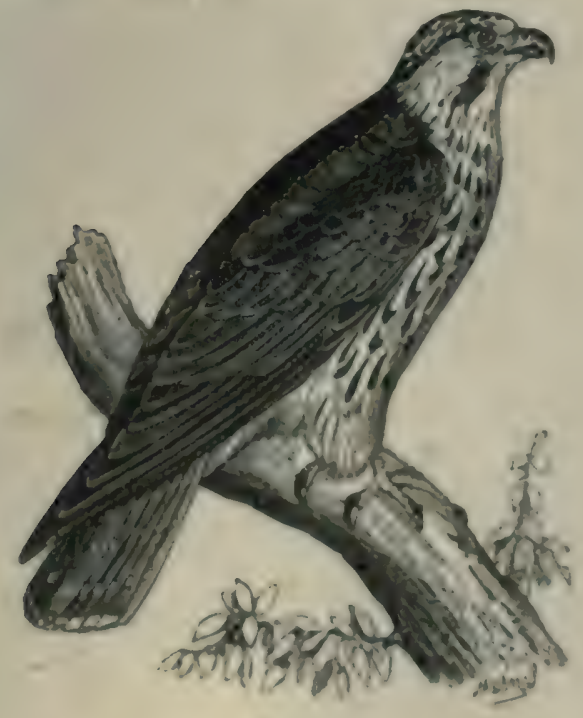

Fameon.

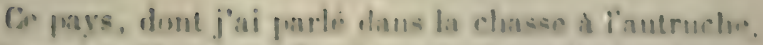

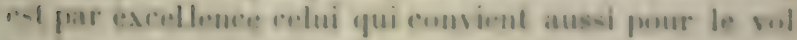

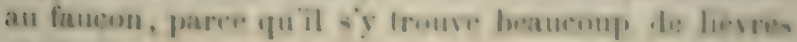
el d'uutardes.

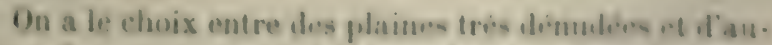

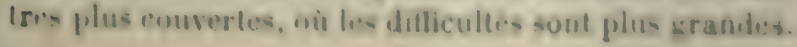


Cies dernières sont quelquefuis préférées parce que;, le gibier s'y défend mieux et qu'il faut ètre caralicr' consommé pour suirre les oiseaux et le lièrre dans un pèle-mile de cavaliers entrainés par des mouvemculrapides, des crochets brusques, avec des obstacles it franchir ou à éviter.

En 1857, j'avais pour agha des Oulad-D'hia, dine-ben-Dhilis, neveu du fameux Ben-Aouda des Oulad-Mokhtar, que j’ai déjà cité comme un des meilleurs fauconniers de l'Algérie.

Connaissant sa passion pour le vol au faucon, je l'avais mis en rivalité avec un caïd des Oulad-Nayls, Kouider-ben-Legbèche, qui prétend n'avoir pas de second dans l'art de la fauconnerie.

Il avait été conven:I, six mois à l'avance, que nous ferions l'hiver suivant de grandes chasses avec les oiseaux de race.

Il m avait en outre été proposé d'ètre juge pour décider qui aurait les meilleurs faucons, de Mahiddine ou de Kouider, et saurait le mieux s'en servir.

Comme cette proposition était grandement dans mes goûts, j'acceptai l'arbitrage.

Pour ètre plus sûr de l'exercer, j'avais eu soin de stimuler les deux biâzes rivaux au moment de la prise des faucons.

L'amour-propre, ce levier si puissant sur le caractère arabe, avait été mis en jeu. - Je faisais savoir par intervalles, à l'un, que l'autre avait capturé plus de faucons, faisait de plus grands progrès dans leur dressage, etc.

Il n'était pas besoin d'employer à outrance ce stimulant, car mes fauconniers, d'eux-mèmes, faisaient merveilie. 
Viahidiline, campi près des Sebaa-Rouss, la muntagnt anx Scpl-Tètes, qui borne an nord le pars des

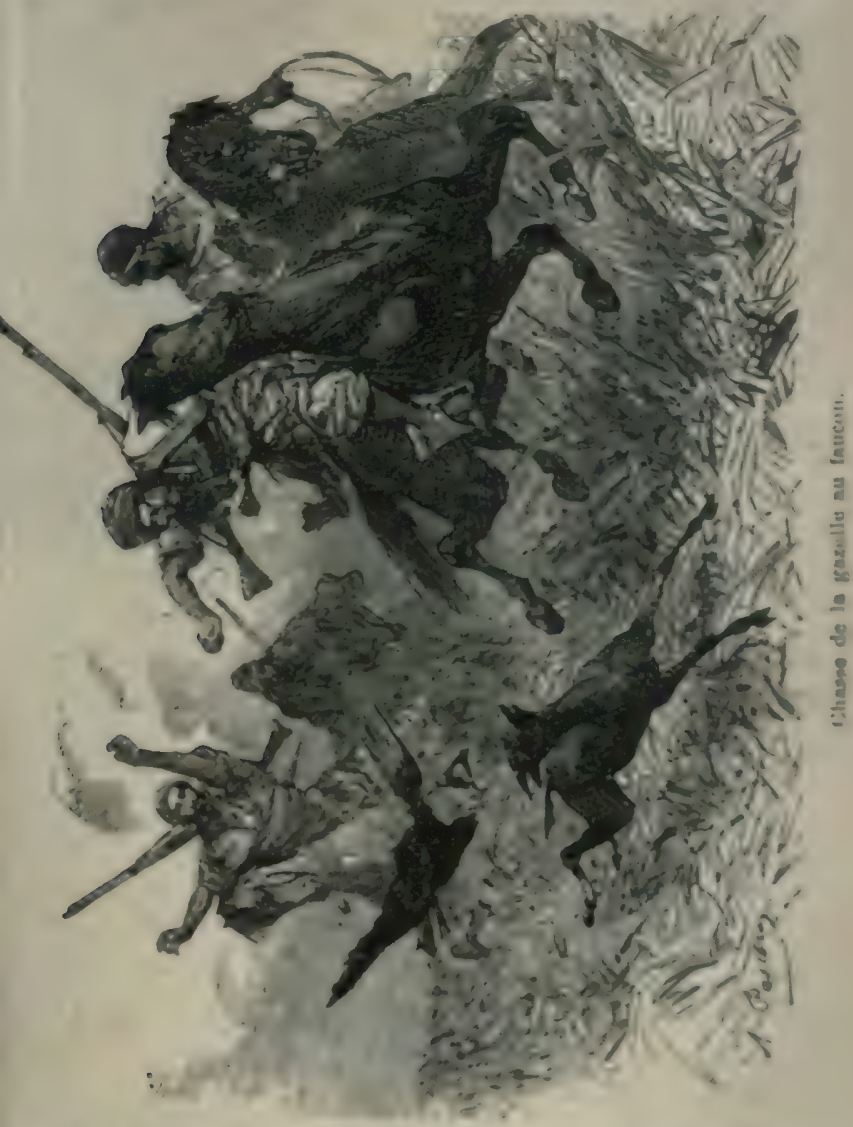

Uulad-Nayls, et à layuelle ce nom a ête donné à cause de sa configuration, - avait pris, avec l'aide de son 
fameux biâze, Mokhtar-el-Meguenni, huit faucons do Ia plus belle espèce du pays; deux, parmi ceux-ci, avaient léjà cha-sé avec Mahiddine l'année précédente, et purlaient sa marque.

Kouider-ben-Legbèche, de son côté, avait pris six faucons dans le pays des daïas, entre Laghouat et 1 . .Izab; - deux étaient des faucons sors, c'est-à-dir. pèlerins ou voyageurs.

Inutile de dire que, dans l'éducation de ces oiseaux, rien n'avait été négligé par nos djouades; ils y avaient mis toute leur science.

Je fus prévenu à peu près en même temps par tou: deux que les oiseaux étaient dressés et ne demandaient qu'à chasser.

J'indiquai aussitôt un point de réunion, en avant dì laghouat, et, au jour fixé, nous nous rencontràmes: sur le terrain de chasse.

Ce terrain, légèrement ondulé de dunes de sablr. couvert de drine $\left({ }^{1}\right)$ par endroits et dans dautres triplat, arec une végétation d'armoise, était peuplé de lièvres et d'outardes.

Notre réunion était nombreuse.

Lè défi que s'étaient porté Mahiddine et Konidrr itait connu, on savait que je devais être juge du mpirite de chacun. - Bor nombre de chefs et de cavaliers "'Citite des Oulad-Nayls et des Larbas avaient demandi: it assister à la grande épreuve.

Les biâzes, de leur côté, avaient une suite respec-

(.) Surte de grand chiendent dont les chameaux et les chevaux font leur nourritme. 


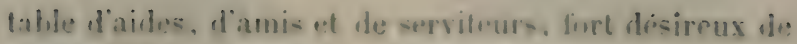
jonir du trimmples de leurs patroms.

Vuns eliuns plu- de soixante cavaliers bien muntes ef plevins diardeur pour la phasse. - Xins avions mos hagitees et $d$ - p provi-inns prour quinze jours.

L.e suir te untre rémion il y eut un gramul ronseil jour adopter le plan de nos opirations ultorieures.

II fut convenu d'aburil que lion ferait sejum le lendromain, que tous los cavaliers et servitours de la suite se mettraient, dans la matinee, a la recherolse ile

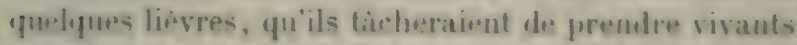
ans gite, afin liasherer ledueatun de trois nu puatre

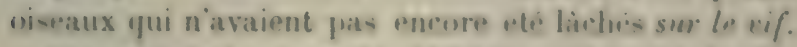

Je remaryuai que cerlle desision, jrise sur la demamd -imultanse des deoux eluefs, Stait me maniere de se titer resiprequement sur la force de leur- fiu-

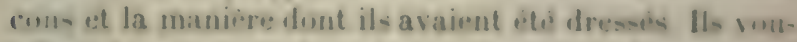
latent de cetle premiere epreus augurer des chame..

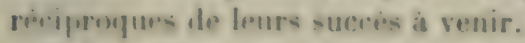

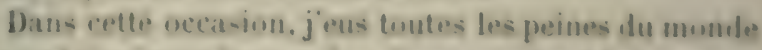

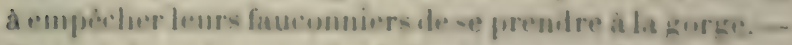
Co sont denx typer qui meritent une anention particulière.

Linn mo numme, cumme je l'ai dit, El-Moklitar-benel-Meguenai. Ciest un formme dont la repulation e-t fort connue dans le Sud, non-coulement comme brase, matis comme voleur andareious.

Oa sail que relle qualices chez los Irabes, quamal elle seserce dans certaimes conditions de dillicultes el do

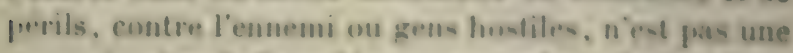
cause de reprobation: licen au contraire, c'est uneloges que l'on fail d'un indisidu quasud un dot de lus : " l'n

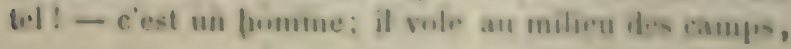


des armées, - il vole les cheraux, les chameaux, etc. "

Effectivement, il faut une singulière audace pour accomplir des rapts au milieu de gens armés, sur leurs gardes, et risquer vingt fois sa vie pour enlever un cheval ou des chameaux.

Le coup de maitre d'El-Mokhtar est d'avoir, dans une nuit, volé $1^{1}$, aux réguliers de l'émir Abd-elKader, lorsque ce dernier vint, en 1840ั, r'azier les Oulad-Chaib, - onze chevaux, - et tué trois cavaliers rouges qui s'étaient mis ì sa poursuile.

El-Mokhtar a quarante ans, sa taille est movenm: mais bien charpentée, les os et les muscles y dominent. Il est très brun, avec des yeux expressifs. Ses traits seraient réguliers si une chute ne lui avait déprimé le nez.

Il monte à cheval de facon à réaliser la fiction dı centaure. Quand il est lancé en chasse à toute vitesse, avec ses faucons aux ailes éployées sur le poing et sur la tête, il passe comme une évocation bizarre dont le tableau de Fromentin rend à peu près l'effet.

Une fois ses faucons lancés sur le lièvre ou l'outarde, El-Mokhtar devient ivre d'action, il encourage ou blâne ses oiseaux en termes énergiques, il oublie toute retenue, et, sans considération pour les personnages avec lesquels il se trouve, il débite un vocabulaire de mots impossibles.

Pour achever d'esquisser l'écuyer de Mahiddine, je vais rapporter un fait qui caractérise sa fanatique passion pour les oiseaux de race.

Un jour, étant à la recherche de faucons à prendre,

(1) Je conserve ici le mot arabe avec l'acception qu'il doit avoir selon eux, car c'est enlevé qu'il faudrait dire en qualifiant l'acte de ce moderne Spartiate. 
il aprent un masniligue cimm ${ }^{-1}$. qui avais en aire flus- uns anfratumsite diun romer lasillo: a pic a huit ou dix métres du sol.

II fallail, pour prendre ce fauron. fembe les laes presde lendruit on il passait la nuil. - Cola stait extri-

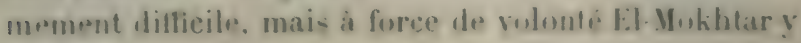
parvint.

Sun nperation toulefois navait ets acherese que tris taril, et ce ne fut que le lemplemain matiu que l'uiseau se prit, en -e jetient at son réseil sur le prismon apprat.

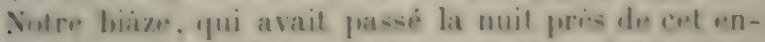
druit et gurltait levemement, ne vit pas plus tit lini-

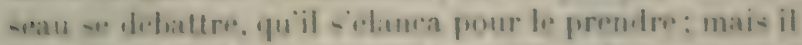
le fit aree tant de precipitation quid feretit tout point d'appui en le sai-i-sant et tomba de la hauteur de laaire du faucon.

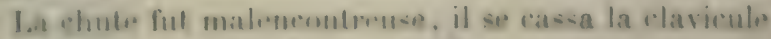

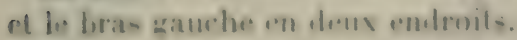

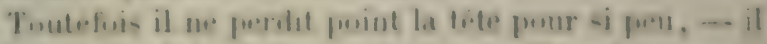

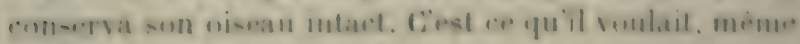

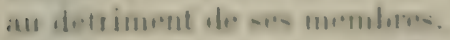

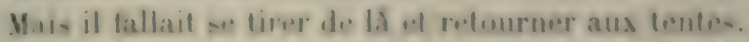

Vl- Muhhlar so mil en marehe, sum loras maude Inal. lane, tonant de la main troite son chere fauron.

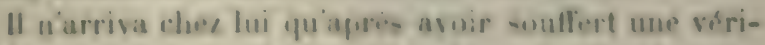
fahle Lorture, - moilie mort de dinuleur et de latigue.

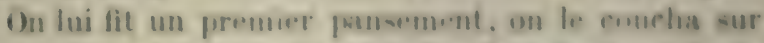

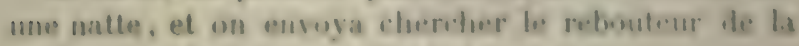
fribu peur lui remellere ses fractures

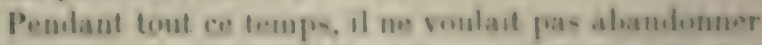
sun wisean; il pressorivil it sa lemmes de lu coudre les

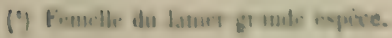


manchettes, de lui mettre un chaperon et de lui attarher les lanières. - Cela fait, il lui dit : "O femme, mits mon oiseau sur mon épaule malade, - c'est le viritable baume pour ma blessure!... - Vois comme il est fort et de belle prestance! - Je n'en ai jamais dressé de pareil."

Il fallut faire sa volonte, et pendant tout le temps de son traitement El-Mokhtar donna son épaule pour perchoir au faucon, qui effectivement devint un véritable phénix dont il se servit pendant plusieurs années.

Aujourd hui encore, il ne parle de son ârem qu'arec attendrissement.

L'écuyer de Kouider-ben-Legbèche était loin d'avoir la vigueur physique de celui de Mihiddine. Abd-el-Kaderben-Sahraoui, ìgé au moins de soixante-dix ans, parlait le moins possible et presque toujours par monosyllabes. - Cétait par gestes et par accident qu'il communiquait arec la gent humaine.

Ses faucons l'absorbaient entièrement.

A force de vivre en leur compagnie, il s'était identifié à cux, et comprenait, je crois, leur langage.

Sa physionomie était devenue celle d'un oiseau de proie : - nez proéminent et recourbé comme un bec d'aigle, yeux gros et saillants, d'un regard fixe et lointain. Les membres du vieillard s'étaient raidis dans la forme de perchoirs ambulants, asile ordinaire de ses oiseaux; ce qu'attestait encore la couleur de son burnous, illustre par de nombreuses maculations $\left({ }^{1}\right)$.

Abd-el-Kader, ancien serviteur des Legbèche, royait dans son patron, Kouider, le représentant de la troi-

(4) Les fanconniers arabes se font honneur de ces traces visibles du port de leurs oiscaux de race. 


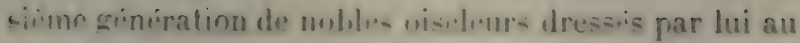
Frand art de famemonerir.

Prendant la rliseu-eien il silrstiut. selon son habiAmt. , de parler: - mais a la tamon dont il regardait

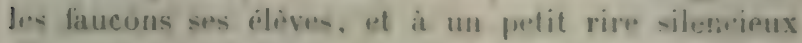
comme celui du vienx trappere do Conper, - qui re-

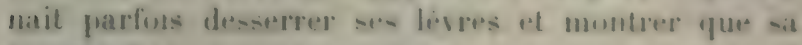

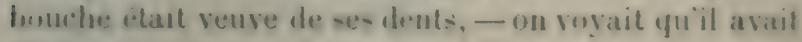
foi dans sa vieille exprerience.

Ce fut El-Mokhtar qui, aver sun humeur tant soit fwu vantarele, commenea lessarmonche: il se buyut rneourage four nous, et se croyait sir declifsecr an rival.

Il commenea d'un fon goguenard le dialugue suivant:

EL-Mukurak. - () vieillard: on dit que tu es savanl sur los wiseatux de race. Je ue suis qu un enfant pros

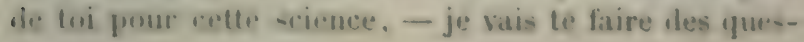
lions pour apprendre

ABD-EL-KADER. - Parle.

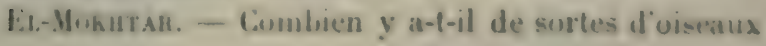
de race?

IBU-EL-KAnEn. - Guestion dienfant!

lil-Mukutak. - Dis luujuurs, je veua savoir.

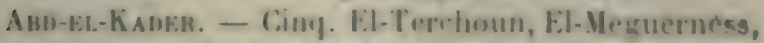
El-Arom, El-krelmui. El-Balıri.

Fi-Mokutan. - Virillard. Iu culslios les Terahifl?

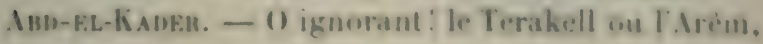
ce n'est qu'un.

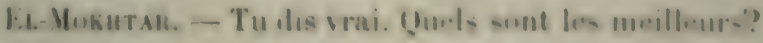

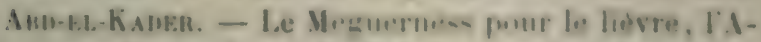

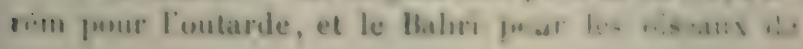
marais. 
Lit-Mozur.ur. - Quels soml les meilleurs faucons? ceux qui fondent de haut rol sur la proic, - ou la suivent et la happent au passige?

ABN-EL-Kinerr. - Question d'âne! - On ne parle yue des oiseaux qui tombent du ciel sur la chasse, et mon de ceux yui la suivent comme des chiens.

I:L-Mokntak. - Avec quelle chair nourris-tu tes oiseaux?

ABD-EL-KADER. - Irec du livre autant que possible, la chair donnée froide au dribut du dressage, et chaude à la fin.

lis-Mulintar. - Combien, ò vieillard! te faut-il de fromp pum lresser tes faucons?

.lbl-el-Kialer, qui commence à ètre faligué de tant cincer, du ton et du peu de portér des questions, rifund d'un air de dédicin : Ma femme suffirait prour ripondre a de parpilles niaiserics. - Il fiut quarant. jours pour dresser les oiseaux, quarante-cinq au plus. Vuilit tout ce que tu sauras de moi."

El-Mulihtar, froissé de la manière dont le traite le vieillard, et crovant l'avoir pris en défaut parce qüil a une autre méthude à lui, se met à rire aux éclats en sécriant: "Ila! ha! ha! le vieux śégare! - Quaranterinq jours pour dresser des oiseaux de race! quelle ulaisante histoire! C'était du temps de notre père Adam yuf lon faisait ainsi. Vous venez de l'entendre, mes seigneurs, le vieux radote, ou bien, malgré son âge. il nest qu'un apprenti, car il est bien prouvé qu il m. me faut, à moi, que huit jours pour dresser n importe чuel oiseau de race. - Perdu le vieux ! il ne sait pas !... Aussi, je vous le demande par la figure du Prophète, quelle prétention a ce vieux pâtre de rouloir tenir tète à nous, gens d'oiseaux de pire en grand-père? Cela, 


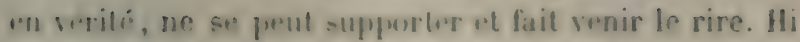
hii hii! " Et lal Muklutar de se tenir les cietes.

Alud-el-kader, se levant furienx et leigayant de coline: " Vous! Tellias 1"! wous n’etes que des enlantprour les vismans, mus fiemmes en sivent plus que vous: - Mes faurons resiennent toujours sur le leurre: Ips noilres sichalpent comme dos chiens qui ont volé un

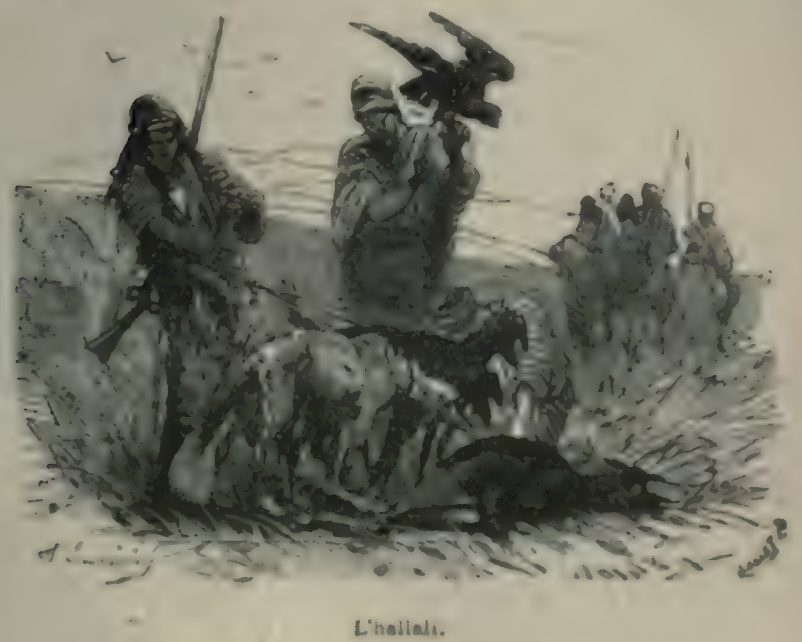

us: je nion fais pas pilus de ras que cola? o Et le bou

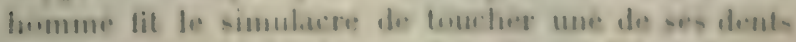

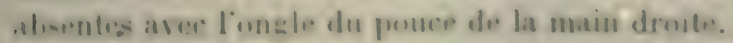

B. Mekntall. - Ah! mes fautens sont des chiens' virux tisom de frut... Tu iusulles mes enfanls: - Vous:

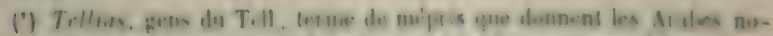

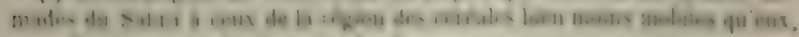

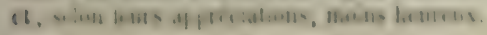


si ce n'était à cause de ta barbe blanche et la présence de ces seigneurs, je rendrais vide la tente de ton piere!...

Arrivés à ce point d'exaspération, nos deux biàzes se seraient fait un mauvais parti, si nous n'étions inlervenus.

" Assez! leur dis-je. Ce n'est pas sur des parole: quion peut juger de votre savoir et du mérite de vos methodes, mais à l'œurre. - A demain donc, et faites la paix arant de vous séparer. "

Je n'obtins qu'arec peine un semblant de réconciliation. Chaque parti, a près cette scène, rejoignit ses tentes. J'entendis El-Mokhtar monologuer en s'en allant: "Ah! vieux berger, je n'ai que des chiens! Tu verras demain, je couvrirai ta figure de confusion."

De son côté, le vieux biàze murmurait : "Cet âne du Tell enfle son ame comme une outre; - certes, je le dégonflerai demain, par la bénédiction de cheikh Abdel-Kader! ”

J'ai raconté celte scène pour donner une idée de Iimportance que les fauconniers mettent dans tout ce qui touche à leur art ou à leurs oiseaux.

Le lendemain, comme cela avait été convenu, nos gens se mirent en quête de lièvres.

Ils en rapportèrent trois, qu'ils prirent virants au gite, avec le bâton fourchu.

Virs quatre heures de l'après-midi, je convoquai Mahiddine et Kouider. Ils arrivèrent arec chacun deux faucons, qui avaient besoin de la dernière épreuve du :ak'relia $\left({ }^{1}\right)$.

(") De $k^{\prime}$ ralli, laisser, - lasser aller sus le vif, sur le gibier qui fuit. 
Le faucon de El-Mokhtar s'éleva de noureau dans l'air et descendit, comme la première fois, sur le lievre qui venait chercher un refuge au milieu de nous, e qu'il manqua à cause de cela; mais à la truisième passe il l'accrocha par la tète et roula avec lui dans l'arène

Ce n'était pas mal pour un coup d'essai. On applaudi fort.

El-Mokhtar, tout joyeux, vint l'arracher de dessus

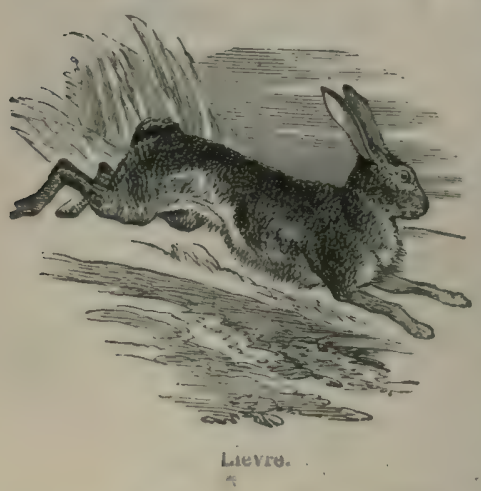
sa victime, qu'il sairna. Il fit boire de son sang tout chaud à l'oi seau, et lui donna quelques becquées de chair palpitante, que celui-ci absorba aver une gloutonnerie de bon augure.

Cela fait, El-Mokhtar lui remil le chape. ron, et revint comme un triomphateur prendre sa place près d'Abd-el-Kader, dont le faucon avait failli et disparu, aux bruyautes hmes des partisans de Mahiddine.

Le vieux semblait leur donner gain de cause, parce qu'il n'arait fait aucun effort pour le rappeler.

La première émotion calméc, je fis lancer le second lièvre et signe un peu après à El-Mokhtar de làcher son autre oiseau.

Celui-ci, moins bon, ou moins avancé en éducation que le premier, vola mollement sur le lièvre, qu'il effleura à peine dans plusieurs passes.

Il était temps de lui enroyer du renfort, car le lièvre 
et lachés en mème temps sur lui quand il aurait gagur quarante pas au large.

L'attention redoubla à celte dernière épreuve, qu était décisive.

Le lièvre fut donc lancé, et les oiseaux aussi lorsqu il eut suffisamment d'avance.

Les deux faucons d'El-Mokhtar volèrent à sa poursurte; mais, n'ayant pas assez d'expérience, ils suivirent le lièvre en rasant terre et essayèrent de le prendre à la nuque avec leurs serres. Ce dernier se défendait en faisant des crochets très brusques qui les déroutait.

Ils n'eurent pas, du reste, à faire de nombreuses tentatives; le faucon d'Abd-el-Kider s'éleva au vent, comme il avait déjà fait la première fois, et, sûr de lui, il fondit de très haut sur le lièvre en venant à sa rencontre. Cette fois le choc fut tellement rude et peu mesuré, que les deux combattants roulèrent dans l, sable : le lièvre tué raide d'un côté, et l'oiseau, de l'autre, évanoui par la force du coup.

Il n'y eut qu'un cri et qu'un mouvement spontané parmi tous les assistants. - Nous courûmes vers les deux adversaires, qui ne donnaient plus signe de vie.

Kouider-ben-Legbèche, qui nous devançait, s'arrachait la barbe de désespoir : " 0 mon viseau! disait-il, je porterai ton deuil. - Qu'est-ce que j’ai fait à Dieu, pour qu'il m'envoie cette épreuve!"

Heureusement le faucon n'était qu'étourdi. Dès que son maître l'eut repris dans ses mains, il rouvril les yeux et revint à lui; quelques minutes après, il n'y paraissait plus.

Kouider et son vieil écuyer en pleuraient presque de joie. Le premier me dit : «Je n'ai pas encore domns de 
nom ì ce fancon. Cios un bahri ' il vient, comme tui, the l'autre coté de la mer: je rais lapyeter, en tun hunneur, le Commandast, - et tu wois qu"il purlera dignement son nom.

Je ripundis que jeétais très flatté. que désormais jo -nivrais avec interet les prouesses de mon hemonyme.

Ce dénouement avait, selon l'expression du vieus liàze, jauni ${ }^{2}$, les figures d'El-Mokhtar et de ceux des siens.

Je relevai le moral de cenx-ci en leur rappelant ques nous navions pas encurecommencé la chasse sérieuse, que l'avenir leur réservait des compensations, que le lendemain surtout derait atre un grand jour.

Ce lendemain désiré par lous arriva enfin. A deux heures de T'aprós-midi nous etions à cheval.

Je répartis notre monde en deux bandes de vingtcimp cavaliers, afin que la trayue put s'effect.ter dans des conditions également avantageuses puur les deux partis.

l.es fanconniers furent places au centre comme ot habilude. Mahiddine aver cing faurons et Konider ave quatre. - Chaque oiseau elait porte individuellement ce jour-la prar les maitres, les lihizes et les aides-fauconniers, afin de pousuir les mettre tous en action sto multanement si on le jugeait a propros.

Nous nous mimes rn marrhe, apros awir pris des

(1) Marin.

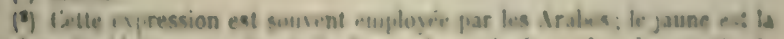
Conleur da d. Bute, de cunt:ason ef de mallewer.

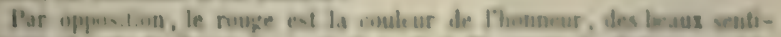

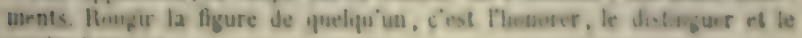
rendre hemrous. 
points de diretion et ètre convenus du pays que nums allions parcourir.

La trayue en ligne par des cavaliers ayant le faucon sur le poing et montés sur de beaux chevaux que l:: chasse anime a un grand cachet d'originalité, qui constitue dejai un spectacle intéressant.

Les interpellations, les cris des chasseurs, les apustrophes adressées aux lièrres qui gardent le gite. maintiennent hommes, chevaux et oiseaux dans éveil surexcité.

" Hé ! un tel, fouille ces toulfes à ta gauche. - Mohammed, retiens ton cheval. - Avance-toi, Ialihdar! - Il n'y a donc plus de lièrres! - Par Sidi-.1is-il, le Saint de Dieu, je n'ai jamais vu un pays aussi vicle:Où se cachent-ils? - Haou, haou! - Brr! Brr! IIé! fils du péché, levez-vous! - Votre jour est arrivé !... - Vous devez finir entre les sabots de nos chevaux et les serres de nos oiseaux. ”

Nous marchions ainsi depuis un quart d'heure, battant l'alfa, le drine et le chihh, lorsque deux lièvres débusquèrent en avant des fauconniers.

Aux cris de : Le voilà! lièvre! lièvre! les biàzes déchaperonnèrent d'abord deux, - quatre, — puis six faucons.

C'était un magnifique lancer. L'alfa était assez. fourni pour offrir des refuges momentanés aux lièrres et dérouter les faucons. Ces braves oiseaux faisaient merveille, excités par les cris de leur inaitre et une ardeur longtemps contenue. Ils s'élevaient dans le ciel avec la rapidité d'une flèche, puis fondaient, comme des aigles, sur les lièvres qui, ayant perdu la tète, couraient en tous sens, cherchant à percer le cercle mourant qui les entourait et à se dérober à la poursuite acharnéc des oiseaux. 


\section{LA CHAST AH FATCON.}

II fant voir lanimation d'un pareil courre, nu mirus d'un pareil vol.

Cavalers, lievres, oiseaux, se croisent, se compent. se herurtront a toute allure, at toute site-ses: - ciet un lourbillon, une course incheveloe, acomplie par des

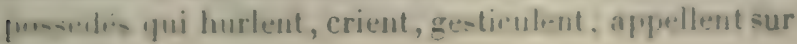
tous les tons.

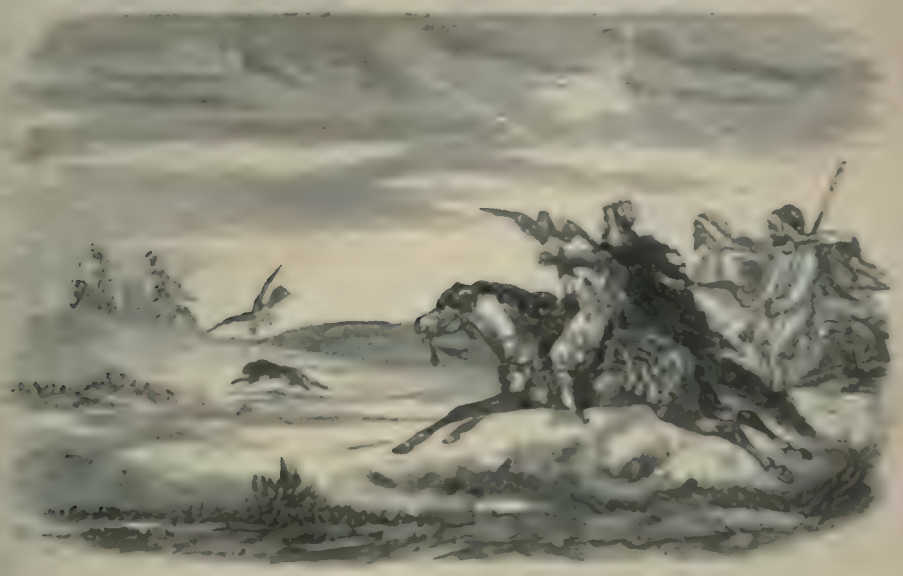

Chasen du lievro es faucon.

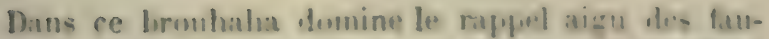

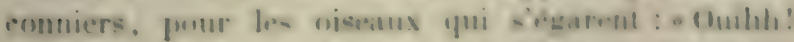
- Uuihh: n et los exclamations de joie quand ils frappent bien :

-Oni! - Oui ! - Force! - Vigupur! a mon sisesau. - mon viseau bleu.... Tu es mon fils cheri! - E.ı

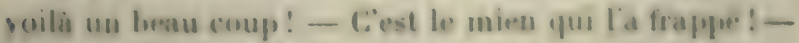
Pour, tu mens, cest le mien! - Si! -... Non ! - Ciest 
Mahiddine ('), te dis-je. - C'est Kouider, le rouge ! Non, c'est le Commandant; c'est l'oiseau marin que rien n'effraye! - Hein! comme il pleut sur le lièrre, dont la destinée est accomplie : - El-Mokhtar, rappelle donc tes chiens qui s'égarent. - Tu mens! ce sont les tiens qui sont areugles et ont besoin que tu aboies pour leur annoncer la curée! - Ici, ines fils, - c'est le jour de la vérité!... - Que les braves se montrent! Ouihh! ouihh! - Haou !... haou !... - Vous n'ètes donc plus mes enfants!... Ah! si, par Dieu! je vous reconnais à ce coup!... Haou !... haou! - Il l'a pris, il l'a pris, mon oiseau! c'est mon oiseau! - Tu ris? c'est le mien qui l'a assommé ! - Non! c'est Mahiddine qui le tient par la tête! - Menteur! - Chien !... au large. au large !...» Et les biâzes et les aides-fauconniers se gourment entre eux pour se convaincre réciproquement.

Nos deux lièrres sétaient fait chasser six ou huit minutes; - ils vinrent plusieurs fois chercher refige contre les faucons jusque sous le ventre de nos chevaux.

Nous étions alors obligés de les pousser du bâton pour les relancer, car l'effroi qu'ils ont des oiseaux est plus grand que celui de l'homme et des autres animaux.

Ils furent pris presque au mème moment, - un par les faucons de Mahiddine, et l'autre par ceux de Kouider.

Le plaisir est d'autant plus grand que les oiscaus chassent longtemps.

C'est un spectacle qui exalte au possible, que celui de plusieurs faucons qui fondent en cascade, l'un aprics l'autre, sur le lièvre.

(') L'oiseau qui parte le nom de son maitre. 
Quelquefois celui-ci est luc du firmier coup, le plus souvent apres plu-ieurs passes de haut en bas.

Il arrive aussi que le lievre est pris de bits vol par un fauron qui rase terre et qui, saccruchant de la serre is la tete, boule avee lui par la firre d impulsion.

I.ess autres faurons arrivent alors a la rescousse, se cramponnent a la malheureuse bete, qui punsere des cris plaintifs; ils forment avec elle une pelote mouvante dans layuelle on ne distingue plus quiun finullid'ailes, de pattes, de plumes et de puil. - I'uiş arrivent les fauconniers qui speparent les combatlants, car lon faucous, animes par la poursuite, sattaquent sunlent entre eux et se dichirent it comps de bere et a élreintes de serres. - Clateun alırs rmpend le -ien ut le chaperonne.

Le fatron qui a tue ou gris le lievre ubtient une

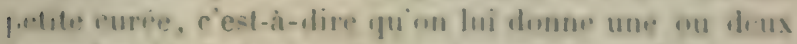

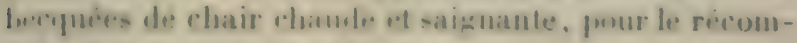
penser et le lenir en hathine.

Quand, ce qui est rase, le lesser parsient a fochap-

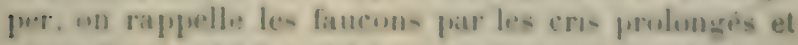
aisus ale: : ouihh! ouihh! et en leur mosatrant ou en leur jetant te leurre, sur looguel dis riennent presigue Loujours se poser quand its sont hion dremes.

Pendaut la chasse, si un aigle apparait. quelque Angue qui il soit du theatre de lartion, on rappulle les

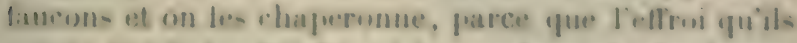
ont de langle les ficit fuir et les rend sonorl-. la phlupart dutemps, aux cris de rappel.

Ausen laigle e-t-il lenne:mi intime de tous los fan-

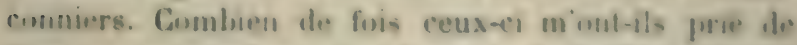
turer sur cens qui venaieut h porthe de fusil! - Uuand jen trais un, il etait mange asee avidte partes hilizes. 
qui l'injuriaient encore après sa mort : Voleur, fils du péché! tu voulais manger mes enfants! - c'est moi qui te mangerai grillé sur le feu!"

Après la réussite de notre première capture, nous continuâmes notre chasse, qui fut très heureuse et très animée. - Vers quatre heures et demie du soir, nous comptions dix-huit lièvres de pris.

Toutes ces prises n'avaient pas eu licu sans contes-

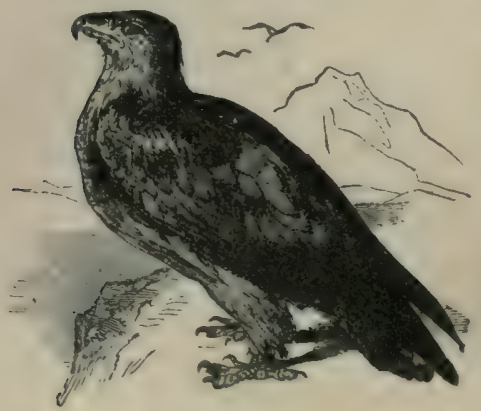

Aigle.

tation:- à propos d'un lièvre tué par deux faucons appartenant, un à ElMokhtar et I'autre à Abd-'el-Kader, ces deux rivaux faillirent en venị aux mains. Ils araient simultanément saisi l'animal par une patte, et, à force de tirer chacun dans son sens, ils l'avaient lacéré et s'en jetaient les morceaux à la tête.

Quand je vins les séparer, je leur fis honte de leur emportement et parvins, à force de compliments répartis le plus consciencieusement possible, à calmer leur susceptible antagonisme.

Comme nous avions assez de lièrres, je proposai de retourner vers nos tentes et de chasser, chemin faisant, quelques outardes que nous avions aperçues pendant la chasse, mais sur lesquelles nous navions pas voulu lancer nos oiseaux. 
Cest un wol que li. - faucunniers nentreprennent jamais sans apprethen-jun.

Tous les faucuns ne sout pris apules a premdre loutarile, yui se defend a torre et qui. par son rol puis. sant. les entraine of les ford sourent.

Mahiduline et kouders, tqui, en giens lien élevés, etaient re-cés en bons termes, malgre l'animosité recipronfue de leurs escugers. se gratlierent lioreille a ma fmom-ition. - Mais comme, en re-umé, le vol à l'outarile entrait dans nutre programme, il fut romermu guion dichaperonnerait pour celui-ci deux fancons serulement, - à la sràce de Dieu!

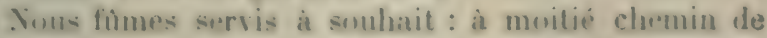

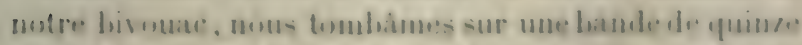

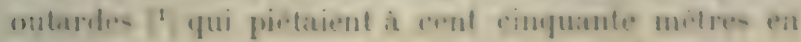
avant de nous.

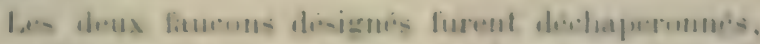

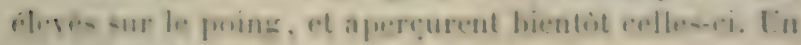
seul fut làché d'abord.

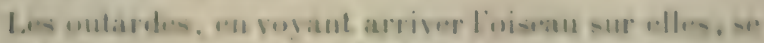

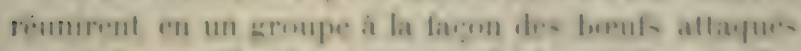
par un loup : elles firent lote en heri-sant heurs colle-

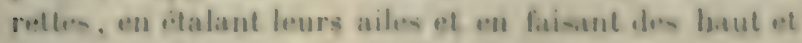

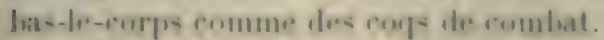

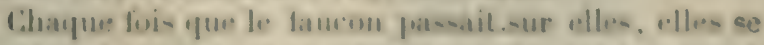
rasitent a terre puur se rellever ensuble et farre face a

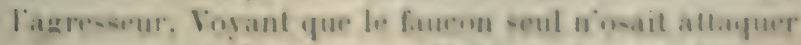

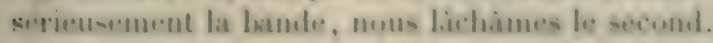

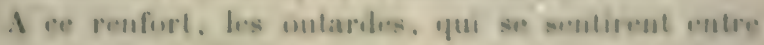
deas attayes, eurmt peur et simulerent dans trutes les directions.

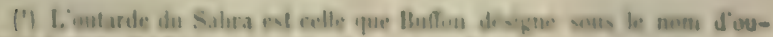

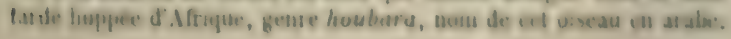


Le premier faucon lâché, qui avait l'altitude et le vent farorable, profita de sa position pour fondre sur une outarde qui vint à passer au-dessous de lui; il fut assez heureux pour lui casser l'aile droite du premier coup de serre, et l'abattre. Elle n'avait pas touché terre, qu'il l'avait saisie par le cou et tombait avec elle en conservant son avantage, c'est-à-dire le dessus.

Ceci est très important, parce que l'outarde a la vilenie de saliv le faucon quand elle l'a sous elle ou it sa portée.

C'est une défense suprême et très efficace que la nature lui a donnée là.

Quand cette défense est employée à propos, le faucon. qui recoit le jet liquide et corrosif, en est aveuglé, ses plumes sont mouillées, et il est obligé de lâcher sa proie.

S'il n'est pas lavé à l'instant avec de l'eau, il est hors de service pour le reste de la saison, la matirire lancée par l'outarde ayant la propriété de coller les plumes et de ternir la vue.

Le second faucon fut moins heureux que le premier; devancé par les outardes, il fit vainement tous -es efforts pour les rejoindre, et n'y put réussir.

Après plus de deux lieues de poursuite, il allait s'egarer quand son maitre, Kouider-ben-Legbèche, qui l'avait suivi à distance, arriva assez près pour lui faire entendre le cri de rappel et lui jeter le leurre.

Il le reprit de cette façon et nous rejoignit après notre rentrée aux tentes, fort satisfait d'avoir reconquis son oiseau.

Le vol de l'outarde offre plus d'intérèt encore que celui du lière, en raison de l'attaque et de la défense, uqui sont plus sérieuses, - et de la rareté de ces ma- 
ynifipues wiseaux , qui ont, avec un superbe plumaze, une chair exquise.

Voila lo rioit de notre prennière jusurnée.

Le surlendemain, nous primes sept outariles.

Co fut notre plus grand triomphe sur ces superbes gallinaros: mais il fut payé chirement : la perte de deax faucons, l'un efrayi par l'aigle. et lautre enPrainé bien loin par la puursuite et ègaré.

Pendant quinze jours. nous avens chassé aver: das chances diverses, mais toujours avec des taptures plus on moins gramles de lievres ou dioutardes.

Souvent jo fus sollicite par Mahiddine et Kunider de me pronomere sur la valeur do leurs fameons.

Cola arrivait, rommone on le devine, toutes les foris

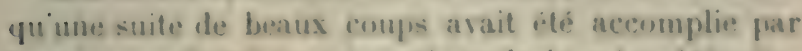
les niseanux do lol un tel parti; mais je men défemdis. remellant tonjours la sonteree derisive a lissue de la rhasse.

Unand elle arriva enfin. je délarai, dans la reuniun des adiens. que Vahidatue el Kendider blaient dee faueonniers imcomparaleles. que leurs wiseaux de race etaient les meilleurs que jarais vus suler: que leurs

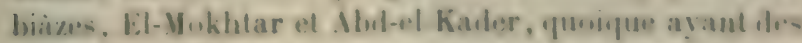

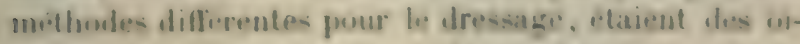
seleurs du fremier merile: enfin que bebes et gens so. taient admirablement conduit-: que mous navionsqua

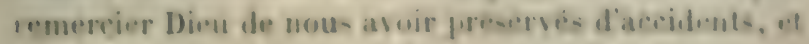
i Jui domander de nous innmer hous less ans une -i Inume sai-un de cluasse.

C̈. itait la vérité, el, de plus, je niaurais pas vumb faire le mécontronts en dounant la prafme a un partiau détriment de l'autre.

Jeus soin toulefwis, en particulier, de nuaucer um 
opinion, et je tiens pour assuré que l'am!our-propre de mes fanconniers aura su en tirer parti pour se décerner respectivement le premier rang; - ${ }^{*}{ }^{*}$ 'est une satisfaction qu'ils n'auront eu garde de se refuser.

En terminant ce récit, j’affirmerai encore que le courre de lautruche et le rol aux faucons sont les chasses les plus attrayantes que lion puisse faire en ce monde.

Elles rajeunissent, disent les adeptes, et je le crois

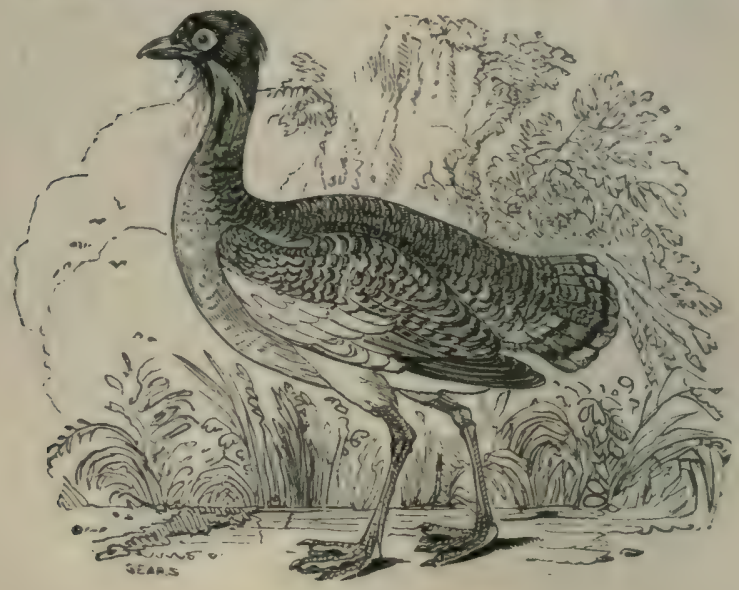

Outarde.

sans peine, en raison du plaisir intense qu'elles donnent, en mème temps qu'elles poussent au maximum d'action toutes les facultés locomotives.

Les fauconniers ont des chants pour leurs oiseaux de race.

Chaque biâze, du reste, est un peu diseur. - Dans les longues nuits du dressage et pendant la chasse, il 
improvise souvent des stances quil chante a haule voix sIr un mole caldence, qui se termine loujours pare le cri de rappest.

En whici un ique jai a peu prìs rebenu dans mes smuvenirs :
Ia flueir et-lweta:
O eiseau de la lutle:
fellown el- leama,
Combattant de liair.
M.toleh not iouha.
Comme tui il we sen truuve.
Ou..in: ousin!
Ouhlh! ou lih!

Nit ar aj-....id

MI : thents sit

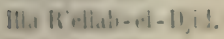

Unah' owiht !
Au juste de la clen...

1! a's a de stignear

Uue Riellal, le Nutsw.

Multh! outh: !

On ain tlienla ia arneb el -m khrua 1.:?

Ou ain elteilit Ja oum el-houbara?

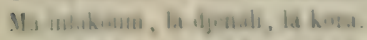

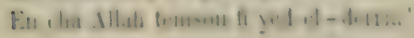

Owith! ovilhb! Haou 1 haow?

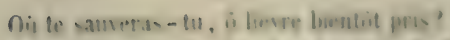

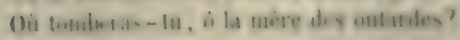

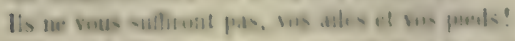

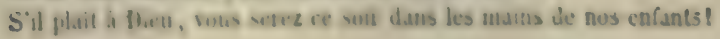

Duilh! ste.

Men, y ferrah benal archi?

Men y bamoucur oudj khrouti?

Men ibièn khrussetet Mhrrilh?

Men, men hermenu dewa heess?

Uuilah! etc. 
Qui donne la joie aux filles de ma tribu?

Qui rougit la figure de mes frères?

Qui fait paraitre les vertus de me's chevaus?

Qui des maux de ce monde donne l'oubli?

Ouililı! etc.

Theiri? their el-Salura el-kerim !

Futhoil Allah cl-adlim!

Nechekerck ĩa ouldi alı el - daïm.

N'haar maik, nem İum el-djenna, ida makount naïm! Ouihh? ouihh! Haou! haou!

C'est mon oisean, l'viseau du disert, le généreux!

Présent de Dieu le fort! le très haut!

Je te louerai, o mon fils, sans cesse ni répit.

Un jour avec toi est un de ceux du Paradis, si je ne suis en rève! Ouihls! ouilh! Ilaou! haou!

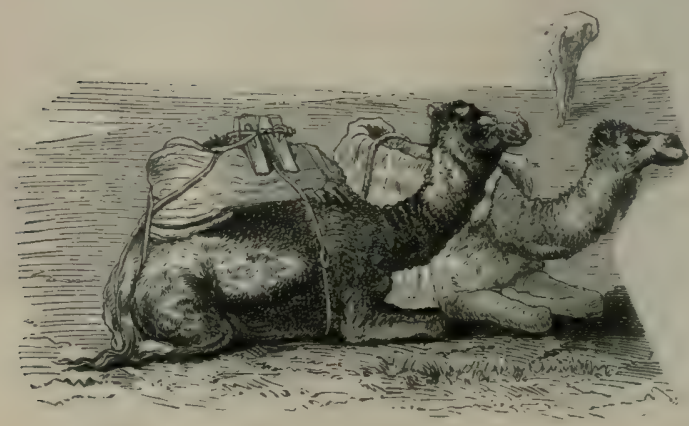




\section{H A II E A U X}

\section{MIRAGE - PRESAGES - DIVINATIONS}

I.ITTE் RATU゙BE ET POE்SIE ARABE

\section{CHAMEAUX}

Parmi les animan pue l'homme a assujettis is an service et quil a faits ces auxilaires dans le pêterinase te lat vie, il $y$ en a de plus parteculatement utiles. dont il ne saurait se passer tlans certaunes ronditions d'exiatence.

Tris sont les chameaus i' prour les prouples numades "- photeurs qui virent dans les régions desertes.

Pour ne parler que du Salira de l'Algerie, que uncolunnes ont traverse tant de furs en sumbant ses mystires, on preut dire plue los chameans y sont en si stancle estime, qui elle approches foe la veneration; ot if fuut reconnaitre ijue re sentiment se trouve justilie par

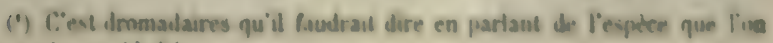
renculotre en Alyerie. 
les services conslants, exceptionnels, que les chameaux rendent à leurs possesseurs.

C'est gràce à ces excellents animaux que les nomades menent cette existence si libre, si indépendante, et s'aflranchissent de certaines misères qui pèsent sur les citadins, mème les plus civilisés.

Certes, il niest pas facile de convainere des Français,

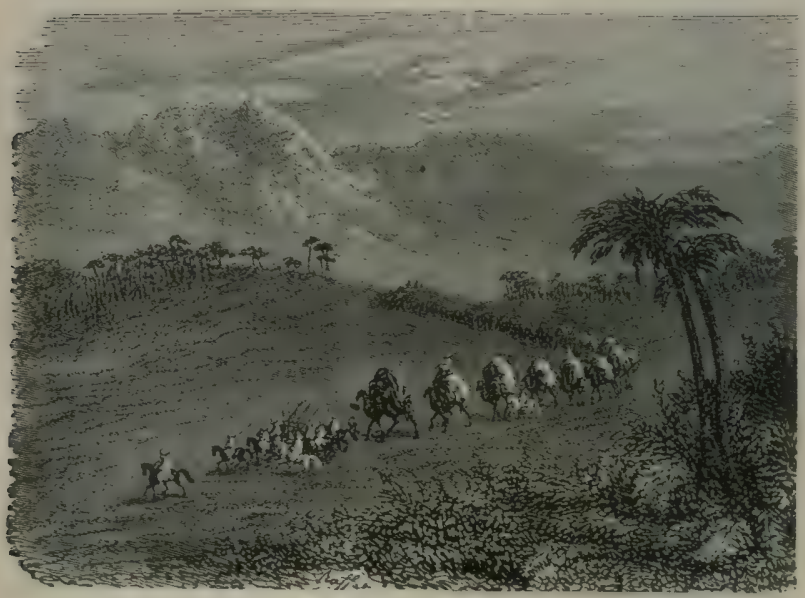

Ciaravane.

des Anglais, des Allemands et tant d'autres. - qui se croient avec quelque raison it la téte du progrìs humanitaire, - qu'il y a un trìs grand charme dans cette antique existence des Arabes pasteurs.

Cependant rien n'est plus vrai : c'est la conviction de tous les Européens qui ont pu en jouir accidentellement. - Tout en reconnaissant qu'ils ne sont peutêtre pas aptes à la mener toujours, ils comprennent l'attachement passionné qu'elle inspire aux nomades. 
Wh hien, celte existence si large, que bien souvent jls préfèrent mourir que de la perdre, les Arabes ne peuvent la mener qu'arec leurs chameaux, seuls véhicules possibles dans le Sahra. - Aussi on comprent que ces indispensables moteurs de la vie au désert soient l'objet d'un culte tout particulier.

Ce sont les chameaux qui portent la tente, les bagages, les provisions, les femmes, les enfants, les vieillards, les éclopés.

Ciest gràce à eux que les Arabes vivent dans le pars de la soif, en les envoyant chercher l'eau à des distances le vingt et trente lieues du point où ils unt planté leur: tentes, et peuvent rester ainsi des saisons entières dans les régions de pacage où s'engraissent et foisonnent leurs troupeaux de brebis.

$\mathrm{Cu}^{2}$ sont les chaneaux yui ront chercher Je blé el lorge dans les pays qui en produisent, - qui chargent. dans les ksours, les tissus fahriqués, les dattes, elc., servant au commerce d'échange.

C'est avec eux que les Irabes se dérobent à l'enneıni par de longues marches, et évitent le plus souvent d'en ètre alteints. Cela faisant, ils donnent leur lait si substantiel pour l'alimentation des gens et des chevaux.

Aussi les chameaux sont-ils en grand honneur chir les Arabes. De tout temps les plus grands poètes leont chantés : - on les retrouve dans les Moallakat $\left({ }^{1}\right)$, dans le poème d'Antar et dans le Coran lui-mème. Mohammed n'avait-il pas une chamelle pour monture favorite?

I.importance de la race caméline depuis cette époque

(9) Poèmes sacrés qui méritèrent d'être accrochés (d'où vient leur nom) a la Koubha vinérée de la Necque, pour leur incomparable beauté. 
est sans doute un pen amoimbrie, mais elle est loin l'alre anfantie, el daus mulre sud il est facile de voir

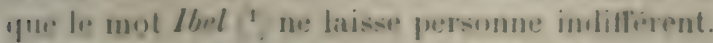

lient une gloire de compuirir les chameaux: iest une honte de les laisier prendre.

Lees plus brares parmi les guerriers tu salura sinurEnosillissent dietre appeles Fersan-rl-Brl, cavaliers dechameaux.

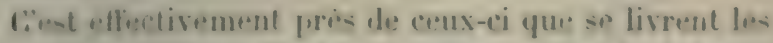

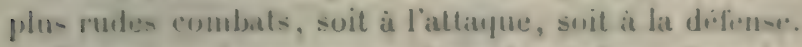

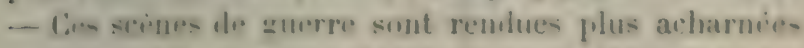

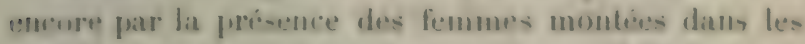

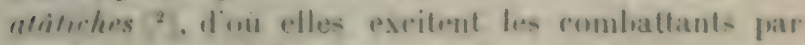
1.0ur- lonatueres nu lesur improbation.

Wuand on dit diun gruerrier: Cn tel samve les cha-

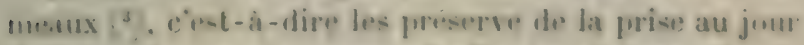

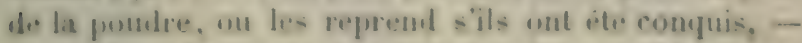

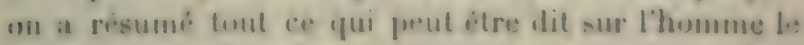
plus brave.

Kleram-el-Bel. voleur de chameans, $\cdots$ l aussi un sus mom elogiras pour colui qui se live a cette aventu-

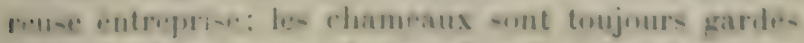
en lempes de guerere par le- cavaliers le- plus vigoureus de la tribu.

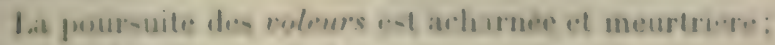
ans s'y bat peor lumt de turt.

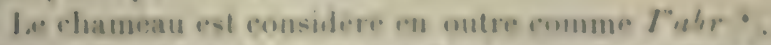

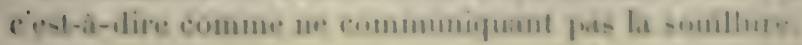

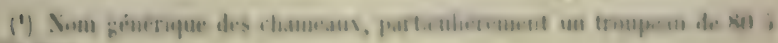
sinotions.

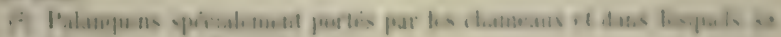
Whireat los fernumes et les enfants.

(1) Nine, Folk-el-Bel.

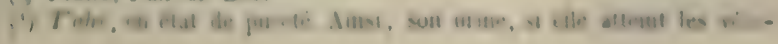


Aux funérailles des grands, pendant les quarante ou

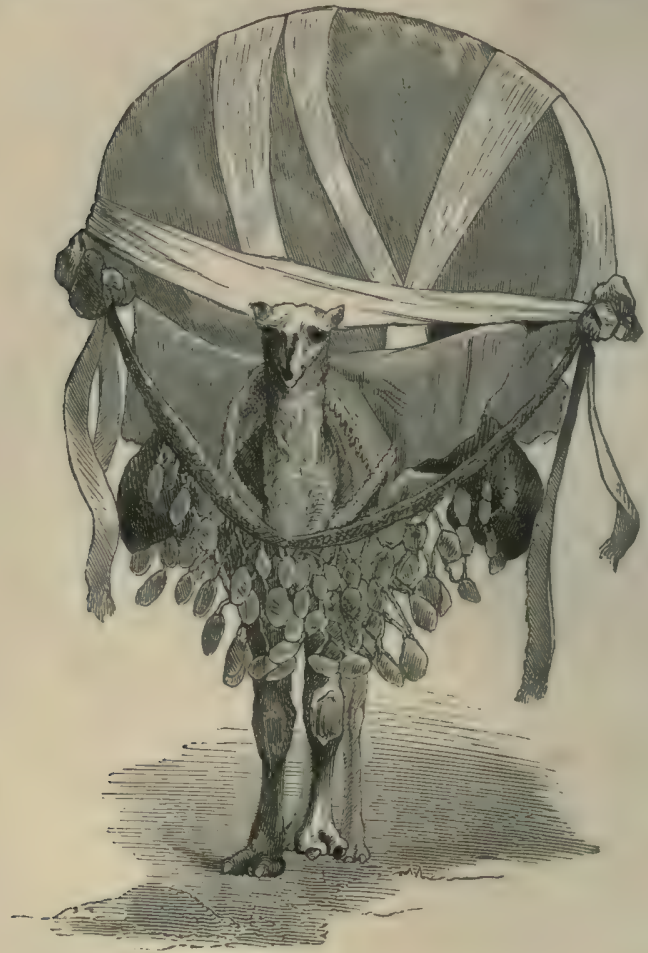

Atatiche.

soixante jours de lamentations publiques qui les sui-

ments de l'homme, ne souille pas, et l'on peut prier avec, sans être obligé de les laver préalablement.

Les crottins de chameaux sont recueillis comme combustible, ils servent à faire cuire les aliments; le feu qu'ils donnent a une grande puissance de calorique. 


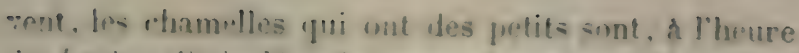

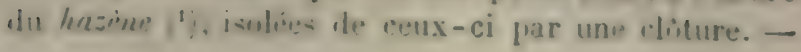

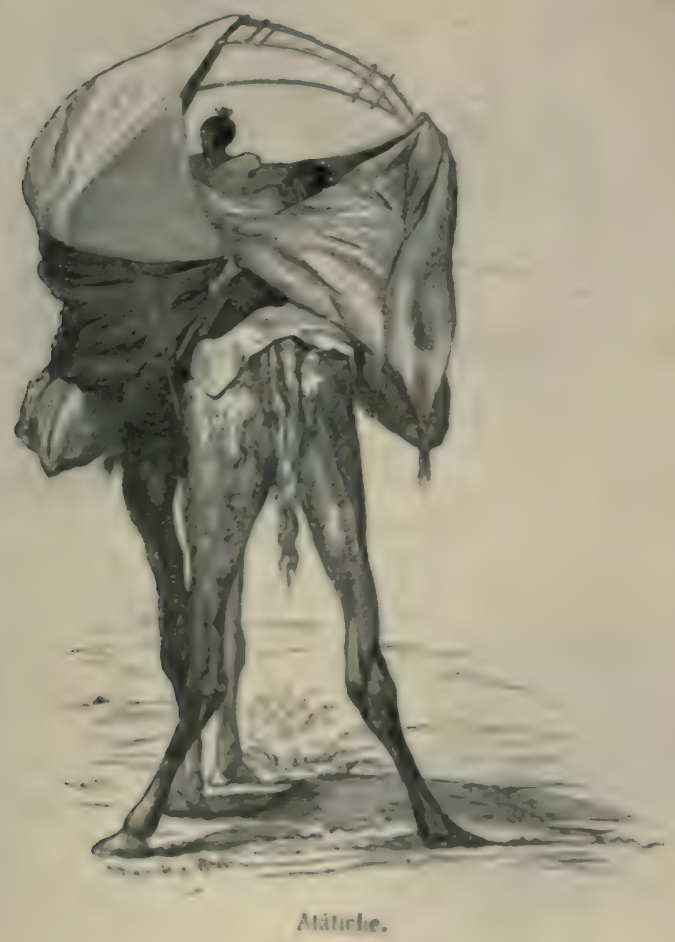

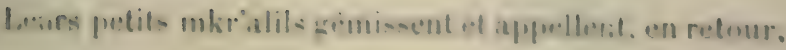

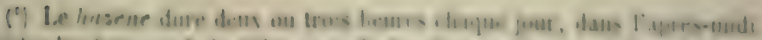

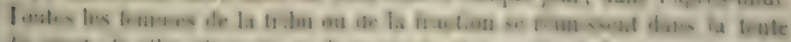

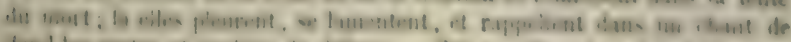

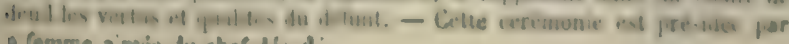
a fomme a inoin du chef the di: 
les cris plaintifs des chamelles, qui pleurent en mème

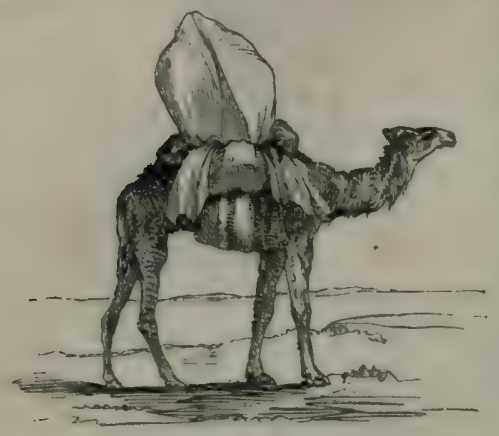

Chameau de voyage. temps à grosses larmes.

Les Arabes, en parlant de leurs chameaux, les considèrent cornme les meilleurs soutiens de la famille. "Ce sont nos amis, disent-ils; ils nous accompagnent et nous aident dans notre existence sur cette terre. Tous nos actes importants. pérégrinations journalières, longs royages, fètes, com-

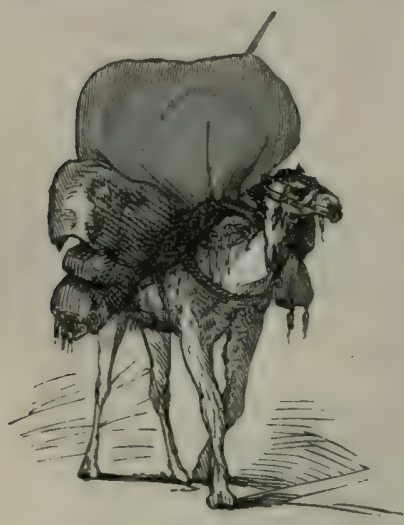

Chameau de royage. bats, fuites, deuil, richesse, - tout cela n'a lieu qu'avec la présence et la coopération des chameaux chéris. "

Ce que je viens de dire du chameau sapplique encore mieux au mahri, qui est áu premier ce que le cheval de selle est au cheval de trait.

Le mahri a mème quelques qualités plus développées, telles que la puissance de locomotion et la résistance à la fatigue; il peut faire pendant plusieurs jours de suite 
jusing trente ou trente-ciny lisues par vingt-quatres heures.

C'e-t avei le mahri que les Tinnareng affrontent le disirt et $y$ vivent. comme un constant problime d. sobriété et d'aventures.

Futin, cest avec les chameaux que mus-mémes avons fu nous rendre matres de la rigion saharmone,

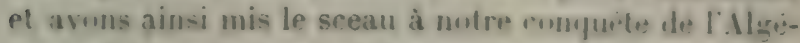
rip. qui nanat jamais etes complot. antroment.

Il y a lout un livre a faire sur les chameaud.

\section{MIRAGE}

Le: mirage a lien partuculierement daus tes grands

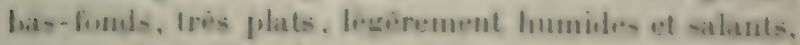
lols que lese chotte, les zagress, - ilden- lee plateaus du Sialia ulgirien, leds que cens du sersun, - et dans

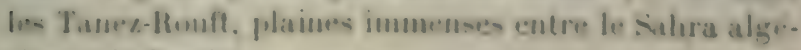
rien et le Soudan.

l.e micage est produit. je crois, par une rondensation vapureuse de hair humbele.

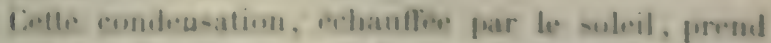
lippareme nuagenes, et forme dimmenses nappes

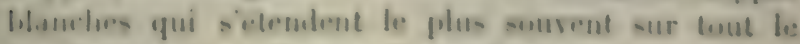

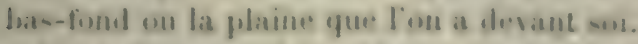

Vu a di-lance diun pumt un peu elowe, le mirage

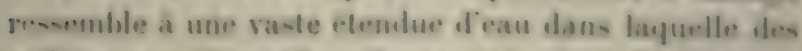
callous ou de peetits arbustes prennent lappareme d thes de furèts igui se deplacent et elongent daspect

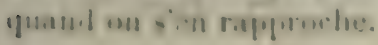


Quand il se troure des animaux dans la partie affec lie au mirage, ils prennent aussi des proportions gigantesques et fantastiques.

Des chameaux que j'ai aperçus une fois à environ trois cents mètres mont semblé aroir des membres de dix mètres de hauteur, et un corps gros en proportion.

Ils semblaient marcher dans l'eau, qu'on aurait juré voir se téplacer par le mouvement de leurs jambes.

Dans les Tanez-Rouft surtout ( $\left.{ }^{1}\right),-m$ ia raconté Cheikh-Atman des Touareg, - les effets du mirage sont prodigieux, parce qu'ils s'exercent sur des plaines sans fin et planes comme une glace : - un crottin de chameau semble ètre une grande tente; des brins de Irine ou de régétaux de la grosseur du doigt prennent l'apparence d'arbres immenses couchés ou debout sur le sol.

Les animaux qui vivent dans la région où le mirage se froduit ne s'y trompent pas; ils n'en subissent pas l'altraction, et je n'ai pas remarqué qu'elle s'exerçât sur ceux qui ne le voient qu'accidentellement.

Je crois, au contraire, que les animaux ont un instinct qui les prémunit contre les effets du mirage et les tentations qu'il peut donner.

L'homme seul, - soit par le fait de son manque de compréhension de certains effets naturels, soit par celui d'une imagination qui aime à rèver l'impossible, - est soumis à l'action attractive du mirage.

Il lui plait de croire ou de supposer qu'il a là, devant lui, des lacs, des îles, des forèts, parce qu'il se trouve dans une zone et soumis à une température où toutes ces choses lui seraient utiles et agréables.

(1) Immenses plaines très unies, dans le centre du grand désert central de l'Afrique. 


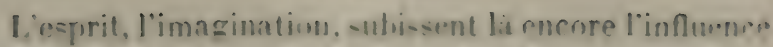

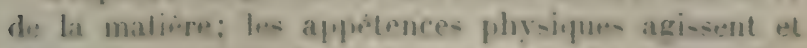

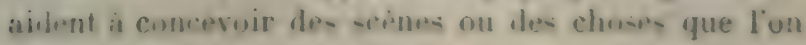
aimerait a voir reillement.

Ausi. tout en ue crovant pas a la rialite des effet: du mirage, on pat puis-amment séduit par les tableaux quil repreicentr. of on sabandonne a lillu-ion.

Combien de firis jai revere, en contemplatant ders effetde mirage, que jotais lautenr de la transformation quss ; opmiait sur une région que je savais ètre torrid. et improductive!

Je prouluisais a ma guise des lars, des rivieres: jo

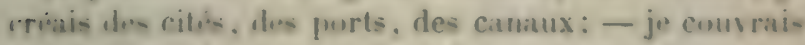
1. sol de forits, de richer moissons, de troupuaux; - 1 , chuse bizarre. qui aidait a la continuation ef at ha pussance de mes illu-inns. le mirage soublait etre a me-s ordres et prouluire, par le fait de ma volonte. lo creations oque jumaginais.

Hirlas ! eient le eas de le dire, - autant en emprortait te rent; un soufllo de Boree me rondait a la malat..

Mais javone que jai trouve taut de clarme ans sinfations ique donue lo mirage, gue je m y sus soumis loutes les fois que jen ai eu loccasion.

\section{PRESAGES}

Tous les penpless sauvages, harlsares ou di demi civiliats, ont une crogance aus choses surnaturelles of a leur manitestation par diverses causes vu movens. 
Les Arabes, quoique musulmans, ont conservé des anciennes habitudes païennes et idolitres de leurs ancètres, drs coutumes, des crovances et des superstitions que la religion de Mahomet n'a pu complétement détruire.

Ainsi, ils sont très accessibles aux présages. Il y en a d'une infinité de sortes et pour tous les actes de la vie. Je veux en citer quelques-uns.

Le présage par les corbeaux est généralement admis quand il s'agit de départ pour voyages, chasses, combats, etc.

Voir deux corbeaux à sa droite est un bon présag̣e; - n'en voir qu un, et à gauche, en est un mauvais.

S'entendre rappeler quand on a commencé la marche pour un départ - est un mauvais signe. C'est la n'echa (1).

Entendre, dans les mèmes circonstances, des mots signifiant joie, bonheur, bien en général, — bon présage.

Rencontrer de jeunes et jolies filles, - bon présage.

Rencontrer une vieille femme, un borgne, un aveugle, un lièvre, - mauvais présage.

Voir, au moment du départ pour la chasse, une haouma, réunion de corbeaux qui décrivent en l'air des cercles concentriques, - excellent présage, signe infaillible de succès.

On pourrait multiplier ces citations à l'infini. Je nai fait mention de celles qui précèdent que pour donner une irlée de cette crovance aux présages chez les Arabes sahariens.

(') De n'echa, retenir, retirer, empêcher. 


\section{DIVINATIONS}

La divination joue aussi un assez. grand rolle sur liesprit crédule des Irabes. La plus connue et la plus ac-

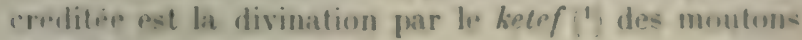
dépouillé de sa chair.

Les devins ou prétendus tels y lisent l'avenir et aflirmont qui un tel sera tuŕ dans telle rempontre, - mourra de telle mort: - que diantres serunt riches. glorienx. ubtiendrunt du pouvoir, - se marieront avec les femmes yu ils aiment, ete.. ete.

\section{LITTERATURE}

$8 T$

FUESIE AHABE DE NOS JOURS

Ia litlerature proprement dite nesel plus en homueur

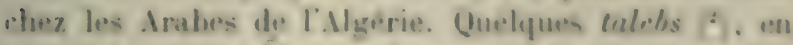
mumbre firt restreint, seuls auteurs de motre cponge. rerivent parfois des notes, des gloses, des commentolaires, sur des questions de droit, de juri-prodence of de religion: mais ces cerits peu connus n'entrent meme pats daus lat cireulation.

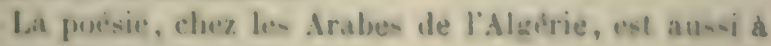

(1) Airter. épanle, amenplate.

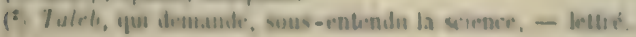


son déclin, quoique l'on puisse dire qu'elle leur soit restée familière et qüils aient l'imagination chaude et facile à l'exaltation.

L'Arabe sent les beautés de la nature, mais ses sensations et sa verve s'exercent plus particulièrement sur la beauté, les charmes de la femme, l'excellence des chevaux, la bonté et le luxe des armes, les faits de guerre, chasse, elc.

Cette poésie se manifeste par des Gals et Ghrazels. sortes de lais.

Quelques-uns s'écrivent, la plupart se chantent dans l'inspiration, se répètent de mémoire, et vont ainsi de tribu en tribu à travers l'Algérie, colportés par les Diseur's, derniers vestiges des anciens bardes et trouvère: d'autrefois.

Ces diseurs se divisent en Gouals, Aiats et Meddah's.

Les Gouals, ou poëtes ambulants, doués du don de l'improvisation, vont de douar en douar, au foyer hospitalier des grands et des riches, chanter, en s'accompagnant de la flûte primitive et du tambourin, les exploits des guerriers en renom, les amours d'amant: célèbres.

Ils fréquentent les marchés, les réunions, les nuces et les fêtes.

Mais comme tout passe en ce monde, ils vont tous les jours s'éclipsant, et, moins heureux que leurs devanciers de l'ancienne Grèce ou des régions du Nurd, les derniers diseurs arabes voient finir avec eux leurs chants et leurs récits héroïques. J'en excepte toutefoi: ceux qui ont été tirés de l'oubli par plusieurs ouvrages sur l'Algérie, entre autres par les livres du général Daumas. 


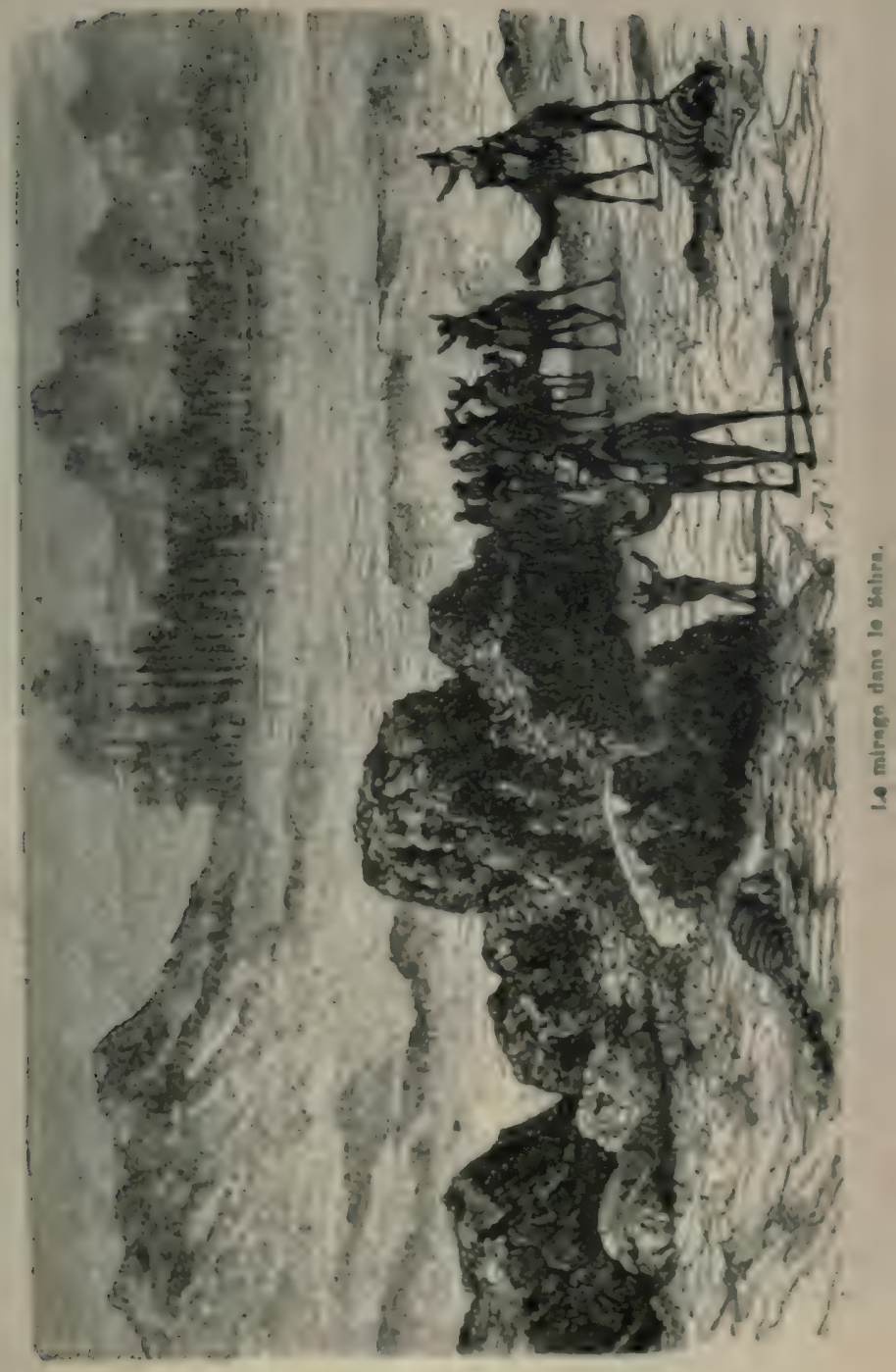





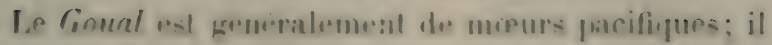
na proint de costume partirulior: il se resonnait neanmoins is une physionomic rèveuse of sousent inspirée. is ses mude-tes instruments quil porte dans le capuchon de son lourmons. On le dísigne allisi sous le nom de Sulnal-ed-s.na ami du metier, de la gnie srience!

L. Aint:' a plus d'analugie avep les bardes belliqueux

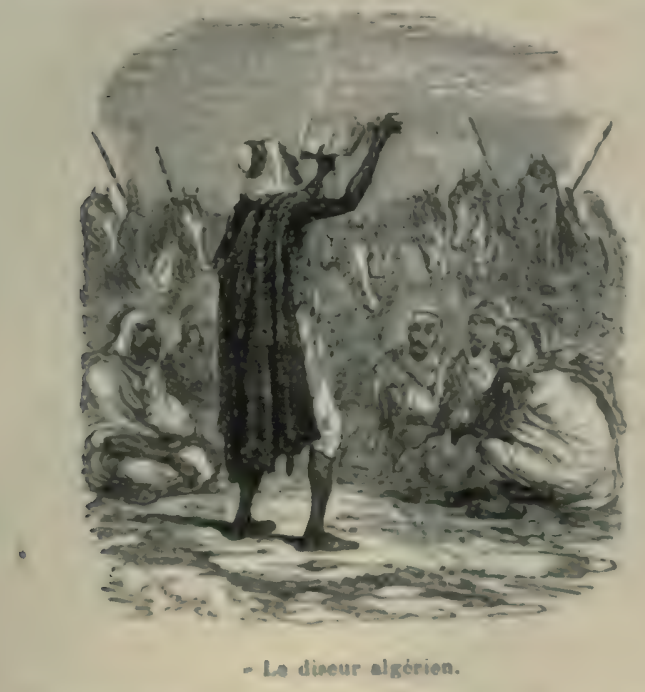

1. la virille Irlande. 'fui savaient aussi binn manier la lourde épée que lo lyre.

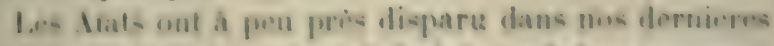
aturress, et eiest a peine si de loin en hoin on en rencontre encure yuelifues-uns dans les trabus guerritres dus siul.

(') De wief, eriet, appeler hauk. 
LAiat, homme de cheval et de poudre, - comme il aime à s'en vanter, - posside une voix d'un timbre aigu, d'une immense portée.

Dans la mèlée des combats, il jelte des appels, des excitations scandées et rythmées, qui exaltent jusqu'à lia frénésie le courage des gucriers.

Véritables clairons humains, - avec leurs voix de cuirre, - ces inspirés de la lutte ont souvent, comme les héros d'Ossian, déterminé la victoire par leurs chants énergiques.

Les cris, les appels des Aïats, agissent sur les nerlavec un effet semblable à celui que nous produit lia charge battue par le tambour: ils doment cette horripilation que l'on définit souvent en disant : "Avoir la chair de poule. "

J'ai été soumis à l'action du chant des Aiats, et mr suis rendu compte de sa stimulante énergie.

Les lambeaux de phrases ou de vers lancés par les Aiats dans les moments décisifs du combat sont des appels aux sentiments élevés, it la gloire des guerriers, à leur's anciens exploits. Quelquefois mème il est fait allusion à l’amour des plus braves pour des beautés en renom.

Après ces indiscrétions, - que l'on peut appeler suprêmes au moment du péril, - un guerrier doit vaincre ou ètre mis hors de combat; il n'oserait jamais se représenter vaincu devant la femme qu'il aime, lorsqu'elle a été invoquée en son honneur pour déterminer la victoire de son parti.

Les Meddah's $\left.{ }^{(}\right)$chantent particulièrement la poésie, religieuse, les prouesses des compagnons du Prophète.

(') Meddah, louangeur; - medh, lonange. 
Le Iedhs, dou ils liment leur men. sont de petils

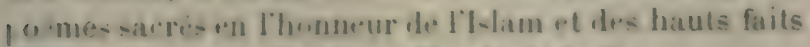

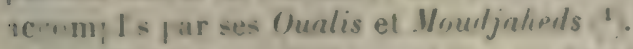

l. s Mediatis so distinguent par un finatisme outré.

P'nétre. et convaincus de la viracite des dires et crotes de leurs herus. de la superiorite de leur rel ginn sur celle der infialeles. ils senisrent a leurs propres

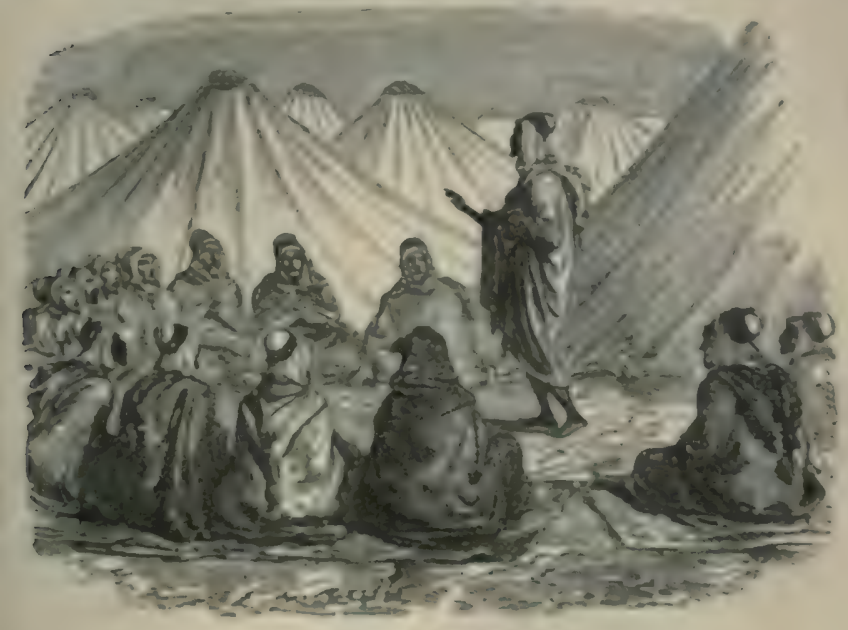

Lo guesal of too enditoire.

chant- et entraiuent lenurs auditeurs par le recit extraurdimaire des faits cel-hores et par le mode sur lequed ils gont rythmés.

Ia calence, munulune au debut, siolenie el heurtee far frogre-sion, fint par operer une surte d'enisrement imystuque, dout leffet ra tonjours cromsant, et entraine jusquia Peslase les sectateurs du Prophete.

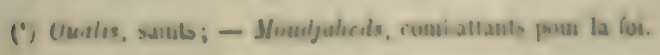




\title{
MESSAOUDA EL-HARZLIA
}

\section{EPISODE DE GUERRE}

ENTHE

\author{
LES BENI-JAGHOUAT, LES LARBAS
}

5. T

LSAR EL-IIIRANE

Avant que notre domination sétablit dans le Sud les tribus nomades et leurs Ksours (1) viraient en guerres continuelles, à peine interrompues par d rares trêves.

Ces guerres ont eu de tout temps pour motifs prin cipaux les rivalités et les questions de prépondérance elles se sont éternisées par les rengeances et les re présailles, de sorte que l'on peut dire qu'elles faisaien partie de l'existence des populations arabes.

Il n'y avait de diversion à cet état d'hostilités ou vertes qu'à l'apparition d'un grand personnage po litique ou religieux, d'un sultan ou d'un chérif, comm

(4) Villes des oasis. 
les Arabes mu donuent si ficilement l. num aux arita-

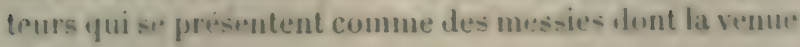
a été prophétisée.-

Mats cette diversion aux querolles intertions orrli-

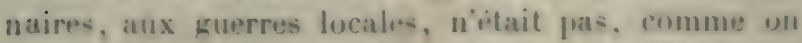
puntrait le supposer, en faveur de? la pais. C:itait une onea-ion, au contraire, pour rendre ce- guerres pluartives et julus génerales.

l.es divers petits partis discidents furmairnt alurs le.s gruupes plus considérables, et, selun leurs intereits un leurs affinifrs, se reunissaient à lappel des nouveaus Sultans, vu restaient du parti local le plus prépund.rant.

II en ri-ultait ainsi un plus grand antagnnisme qui. en are concentrant davantage par le numbre dos indiviluset les passions mises en jeu, amenait un paum ysme dhumeur belligueuse of dactions forergiques remarquables somvent par des combats singuliers. - do

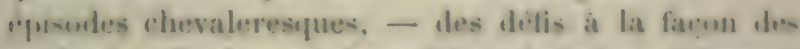
ancilons, of finalement des meleses gromerales, wi le parti vaimpurur etait sans pitie punr le parti vaimen.

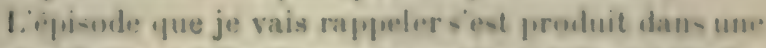
de lees lutles ou les ksours. appeelant a leour atide le.

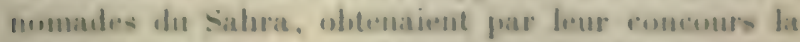

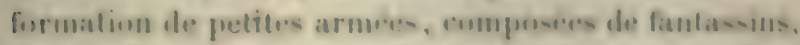

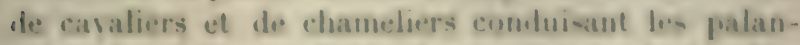

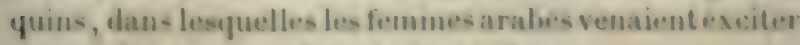
an comblait les guerriers de lecur tribu.

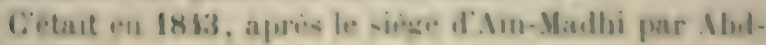
el-hiader.

On sait que liomir, niayant ju prendre la sill. sainle?

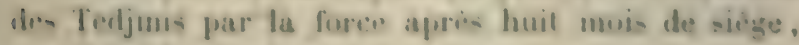
alail use diune founte pour y entrer. 
Il sétait arrangé de façun à faire savoir à Terljini qu'il ne pouvait s’eloigner d’ Iïn-Madhi sans ètre entré dans cette ville avec ses troupes - et avoir fait sa prière dans la grande mosquée.

Il s'était, disait-il, engagé par le serment de ses femmes $\left({ }^{1}\right)$, dont la mort ou le succès pouvaient seuls le relever.

Tedjini, en sa qualité de marabout, comprenint parfaitement l'importance d'un pareil serment, craignant aussi peut-ètre la chute possible de la ville apros quelques mois encore de blocus et de tranchée ouverte, fit faire à Abd-el-Kader des propositions que celui-ci accepta, et qui étaient celle-ci :

Abd-el-Kader se retirerait avec son armée it ElGhricha $\left(^{2}\right)$;

Lorsqu'il y serait arrivé, Tedjini évacuerait lïnMadhi arec sa famille, les défenseurs qui avaient soutenu le siège, et se retirerait à Laghouat;

Abd-el-Kader reviendrait à Ain-Madhi, y ferait ses dévotions à la grande mosquée, respecterait la ville. les jardins restés intacts, eu un mot, se conduirait en ami plutôt qu'en ennemi;

Ses dévolions faites, et conséquemment son vou accompli, il devait quitter le pays avec ses troupes et laisser désormais en paix Tedjini et sa ville.

La convention 's'exécuta en partie, c'est-à-dire qu'Abdel-Kader, après l'évacuation de la ville par le marabout, y revint avec son armée; mais il y revint avec la honte d'une retraite subie, et confus d'avoir échoué

(1) Le serment par les femmes est celui où le musulman jure que ses femmes lui seront sacrées, c'est-à-dire qu'elles ne seront plus siennes, si telle ou telle chose n'est pas accomplie par lui.

Il est obligé de les répudier si cette close ne s'accomplit pas.

(2) Ksar du Ijebul-snour, distant de 8 lieues d'Ain-Madhi. 


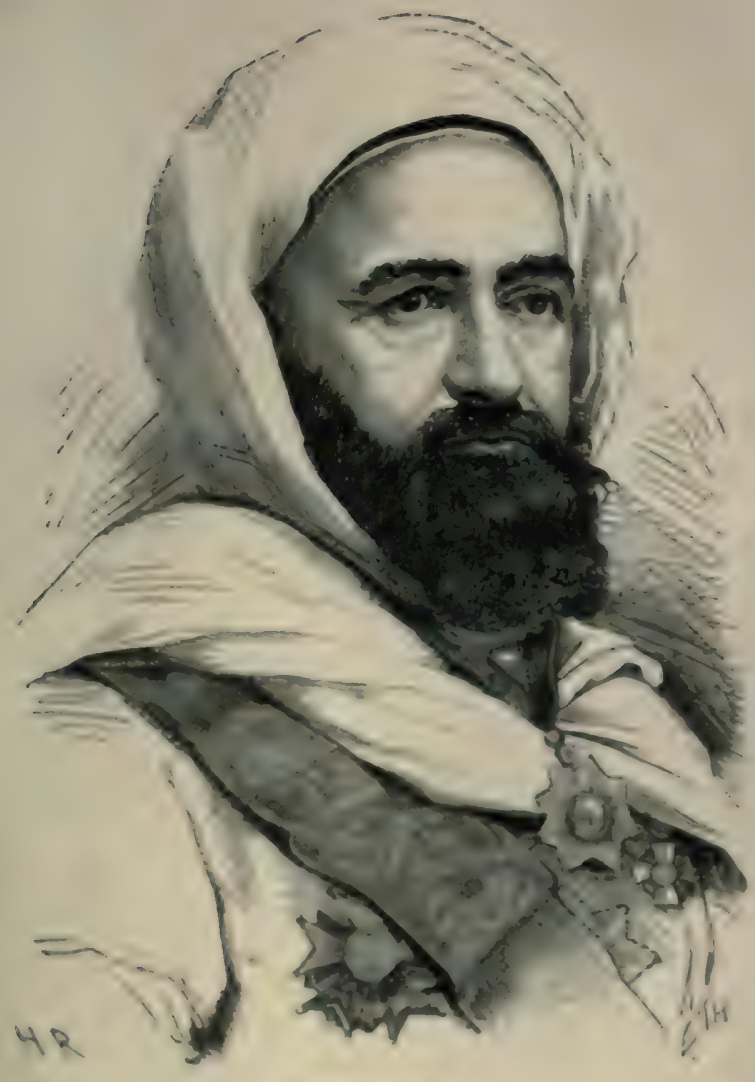

Abd-el-rader. 
devant une bicoque défendue par des pàtres et des Tolbas efféminés, comme il appelait les défenseurs d'Aïn-Madhi.

Dans cette disposition d'esprit, il céda aux suggestions de ses lieutenants et de ses troupes : il rasa complétement la ville et détruisit les jardins restés intacts pendant le siège.

Iprès avoir accompli cet acte de vindicative desLruction, Abd-el-Kader songea à retourner vers le Tell, nù le rappelaient de sérieuses complications.

Il voulut toutefois, avant de quitter le Sahra, lui donner un semblant d'organisation, pour faire croire à la conquête réelle de celte rigrion.

A cet effet, il nomma khalifa du Sahra Si-el-Hadjel-Arbi, descendant du fameux Si-el-IIarlj-Aïssa, l'auleur des prédictions sur l'arrivée des Français en Algérie, dont la koubba ( $\left.{ }^{1}\right)$ est à Laghouat.

Il lui laissa, pour assurer son autorité dansle désert, denx compagnies de fantassins réguliers, une pièce de canon, des k'rials et mekr ezen ${ }^{2}{ }^{2}$.

El-Harj-el-Arbi; qui vivait antérieurement en hostilité avec l'influente famille des Oulad-Zanoun de Laghouat, avait rallié $\mathrm{Abd}$-el-Kader lors de son entrée dans le Sahra; il l'avait servi avec zèle, pendant le siège d'Aïn-Madhi, tant de son influence personnelle que de celle de ses clients.

Il était donc, par ce fait, devenu l'ennemi du marabout Tedjini et de ses nombreux khreddems $\left({ }^{3}\right)$. Il était

(') Minaret renfermant un ou plusieurs tombeaux.

( $K^{\prime}$ 'rials, cavaliers, troupe régulière à cheval créée par l’émir. Mekr'ezen, cavaliers auxiliaires attactús à l'administration du pays.

( $\left.{ }^{3}\right)$ Khreddems, serviteurs religieux affiliés à un ordre. Celui des 'Tedjinis est un des plus considérables dans le sud de l'Algérie. Il étend son action jusque cliez les Touarey. 
de plus considire comme un intrirant ambiticus par les tribus saharienues, pour avoir pactise avec un sultan du Till t:

La purition de si-el-Harj-el-Arbi, après le dipart d'Mbl-el-Kader pour le Tell, fut. comme on le comfirend bien, tress ditlicile.

Malgré sa qualite de marabout descendant de Si-elIladj- It-ca, il n'obtint, en retour de ses avances. yue haume et mauvais vouloir de la part de limportante tribu des Larbis of de la population de Laghouat, pour avour aiité à la ruine de la Zaouia du marabout vénéri d'Ain-Madhi.

Il se seutint quelque temps d'abord dans laghouat. aver l'aide de ses ríguliers.

Mais, harcele chaique juur par la plus grande parti. die la population de la ville et par le- goums des larhise, ne recevant pas de secours d Il d-el-hader qui . on at moment, elait fortement premer par nous, il ferrolit peu á peu son pre-tige et son artion.

Force lui fut alors daller se refugier à k-ar-el-ll.

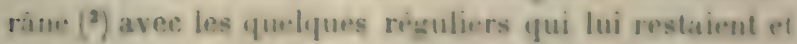
un parti de nomades ef de ksouriens compone de HI. ruzlas, de Ileudjaje et de Raluman.

la situation du hhalufa du Sitora me tarda pas a siz. graver encore.

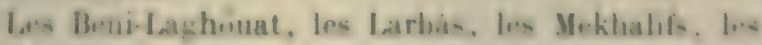

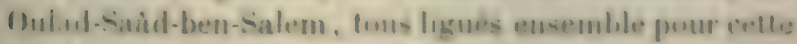

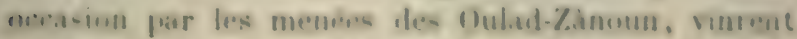
lasmatlir dans sun dernieg asile.

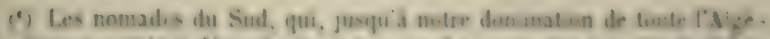

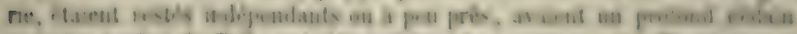

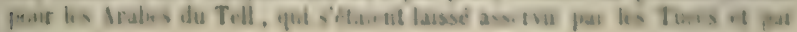
(1) เи।

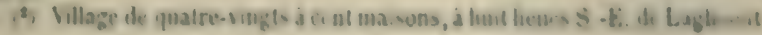


Ce ksar, comme tous ceux du Sud, était entomrí "lime enceinte bàtie en mottes de terre cuites au soleil. Il n'avait aucune autre défense et ne pouvait résister longtemps à une attaque sérieuse.

L'ardeur des assiégeants était extrême, celle des assiégés n'était pas moindre; il y allait pour eux de la vie : ils savaient qu'ils n'avaient aucune merci à attendre de leurs ennemis.

Ils se défendaient en désespérés, et étaient soutenus, comme il n'est pas rare de le voir dans ces combats pntre Arabes, par leurs femmes, dont quelques-une: clonnaient l'exemple de l'abnćgation la plus complète de leur existence en se mêlant aux premiers rangs des combattants lorsque l'assaut était donné aux murailles.

Une jeune fille, entre autres, de la tribu des Нarazlias, se faisait remarquer par sa vaillance; elle se nommait Messaouda.

Cette jolie fille de dix-huit ans possédait une beauté remarquable, éclose et dorée aux rayons du soleil du Sud. Elle avait une taille élevée et élégante, de magnifiques proportions. Elle se distinguait surtout par lexaltation de ses sentiments pour le triomphe de son parti.

C'était une façon de Jeanne Hachette orientale; mais si elle pouvait sous ce rapport être comparée à l'héroïne du siège de Beauvais, elle était peut-ètre moins austère. Elle avait de nombreux admirateurs parmi les guerriers des Harazlias, et s'en faisait gloire. Sa beauté était chantée par tous les ménestrels du pays.

Messaouda, en un mot. était généralement aimée; son action sur les siens était sans bornes; elle devait bientôt s'en servir pour le salut de tous 
Karar-el-Hirane etait as-iren dijit depuis plus dump semainse. Lorstu un soir, apris une jumrnes de combals dans lespurels la firtune atait restee indecise comm. dans les jours précident - les guerriers des Larbâs, deIBeni-layluobat et des Mekhalifs risulurent d'en tinir aser les assisgés par un dernier effort.

Ils se rassemblent de nouveau a cet effet. Encourages par leurs chefs. par l'appat du butin et de la vengrauce, ils se ruent sur les murs de la ville avec des cris de difi et des chants de victwire, - ce qui est tonjour, wntre Arabes, l'inlice d'une affaire sérieuse.

Las defenseurs du Kasr-el-Hiràne, bien moins nombreux que les asciaillants, plus faligués de la résistance que crux-ci de lenrs attaques, recoivent le chose du mirus qu'ils peuvent: mais, apres awir repouse les premier assant, ils sont ohligés de cobler au nombre des altayuants qui se remurelle sans cesse.

II abandonnent la definse de lenceinte, particulispment a un endroit ou une sorte de brèche avait etó ouverte par un thot dassaillants.

C̈en elait fait du knar et de sess défensenurs, si ce premer elisn ent emblinus.

Un connait la maniere de combattre ders Arabes: quand la ticle de coloune linche pried, tout cirle.

Mais ausil, qugnd le surris de ceux qui sonten avan!

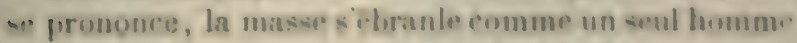
.l sep presipite comme un torrent irresi-lible.

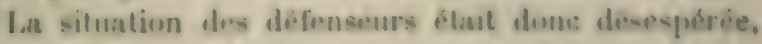
quant la jeume Massamuda, attiree par le fen et les inciférations des vaimpueurs. arrive sur le lieu du combat.

II un eoup dioil elle woit less siens, mis en deronte. ahandunner la defense: elle wit les gurrriers des Lav- 
bîs et des Beni-Laghouat se précipiter sur la brèche, en hurlant des menaces de meurtre et de pillage.

Saisie alors d'une exaltation causée par la honte et la douleur, animée d'une sublime résolution, elle s'élance au-devant des fuyards, les interpelle d'une roix vibrante, et leur jette à la face de ces paroles qui ont tant de puissance sur les hommes d'une nature génireuse, et réveillent toujours d'un moment d'effro: ou de torpeur:

"Où courez-vous, fils des Harazlias! L'ennemi n'est pas de ce côté, il est derrière vous, il vous chasze comme un troupeau de brebis!... Vous abandonnez vos femmes et vos enfants à la rage de ces chiens de sang... 0 jour du deuil noir!... il n'y a plus d'hommes de la race de Harzallah!... il faut que ce soit une femıne qui vous fasse souvenir que du sang rouge coule dans vos veines! $)$

Dénouant aussitôt sa ceinture et la faisant flotter comme un drapeau au-dessus de sa tête, elle redouble d'apostrophes véhémentes qui remontent tous les coulrages; elle s'écrie: "Oủ sont ceux qui disent des chants d'amour pour moi?... - Où sont mes frères?... - C'est ici que je les aimerai!... - Qu'ils se montrent!... qu'ils me suivent!... s'ils ne veulent me voir devenir la proie des jeunes guerriers des Larbâs qui se vantent déjà de posséder vos femmes, vos enfants et vos troupeaux! des Larbâs qui veulent vous faire filer la laine et cuire leurs aliments!"

Puis, joignant l'action aux paroles, elle se précipite au milieu des assaillants.

Peindre la confusion, la douleur et la rage qui s'emparent des guerriers des Harazlias, n'est pas possible. Ranimés par les paroles et par les gestes de la jeune 
hriroine, ils font whlte-liace, shipledtent les uns les an$11 \%$, s exalfent an souvenir de lenrs anciens expluits. quils énumirent a haute wix, et se rejettent it la suite i. Messaouda au plus epais des rangs ennemis.

La sengage alors une de can molopes ou les furces so" d.cuplent, ou l'on lait arme et prujectile de tout, où larme à feu cède le róle a l'arme blanche, ou cefle-ci, lientiot insuflisante, a pour auxiliaires les pierres, les debris de la briche et, dans les luttes corps at corpes, Ins cuuteinx (1), les dents et les ongles.

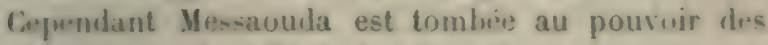
Larbàs, qui veulent l'entrainer vers leur camp.

Llle se prète a ce inouvement, elle laccélire meme en se jetant de lautre cote de la breche. - Sun but e-t dattirer la lutte sanglante rn dehors de lenceinte qui protège lés siens.

Arrive a vingt pas des murs, fle se retourne vers cend quielle a if mergifument ramenes au combat.

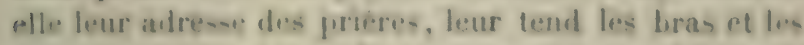
conjure, par thut ce yuils aimut en ce monde, de ne fas la lai-ser emmener par los enurmis, - matir la funte of le mejoris de leurs femmes.

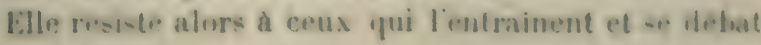
de leurs étreintes.

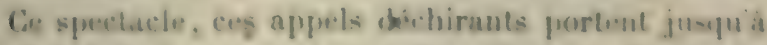
In frenésie fo courage des Rara/lass. - Ruzisane comme des tigres et boulis-ant comme ces pusants animaux, sans tenir comple de leurs lilessures nit de la mort yui les attent, ils remorsent et foulent aus pioculs l.urs alversaires yui, de vainqueurs quils elasent.

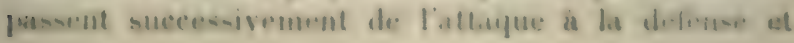

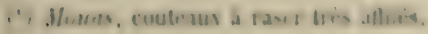


enfin à la fuite. Ils cident it une force surhumaine.

Dans leur retraite précipitée, les Larbâs et les BeniLaghouat essayent d'entrainer l'enthousiaste Messaouda.

Mais celle-ci, qui résiste maintenant autant qu'elle s'est laissée emporter d'abord, est enfin rejointe par ses frères, par ses amis, et c'est autour d'elle que se portent les derniers coups, qui décident une complète victoire en faveur des défenseurs de Ksar-el-Hiràne.

Ce que jaai dit de la facilité qu'ont les Arabes à fuir yuand les premiers combattants sont repoussés, explique comment les Larbâs et les Beni-Laghouat, après avoir été vainqueurs d'abord, virent leur triomphe se changer en déroute lorsque leurs plus braves guerriers eurent été culbutés par les Harazlias.

lls perdirent beaucoup de monde ce jour-là, parce qu'une sortie générale des assiégés vint achever leur défaite.

Quand le combat fut terminé, tous les guerriers des Harazlias restés valides, qui s'étaient distingués dans cette brillante action, ramenèrent en triomphe, au milieu d'une fantasia bruyante, leur bien-aimée Messaouda.

Les femmes et les filles rinrent à sa rencontre, elles lui baiserent les cheveux, les yeux et les mains en lui disant: "Tu es bien véritablement Messaouda ( $\left.{ }^{1}\right)$ ! C'est à toi que nous devons d'ètre encore les femmes de notre tribu. - Que Dieu te bénisse, te rende heureuse et féconde! "

Ce fut à qui la fêterait et immolerait un mouton en son honneur.

La victoire de Messaouda fut bientôt connue dans

(4) Messaouda signifie fortunce. 
tout le Sahra. La jeune fille se vit glorifiée par tous, sans distinction de parti nu d'origrine. Un chant de

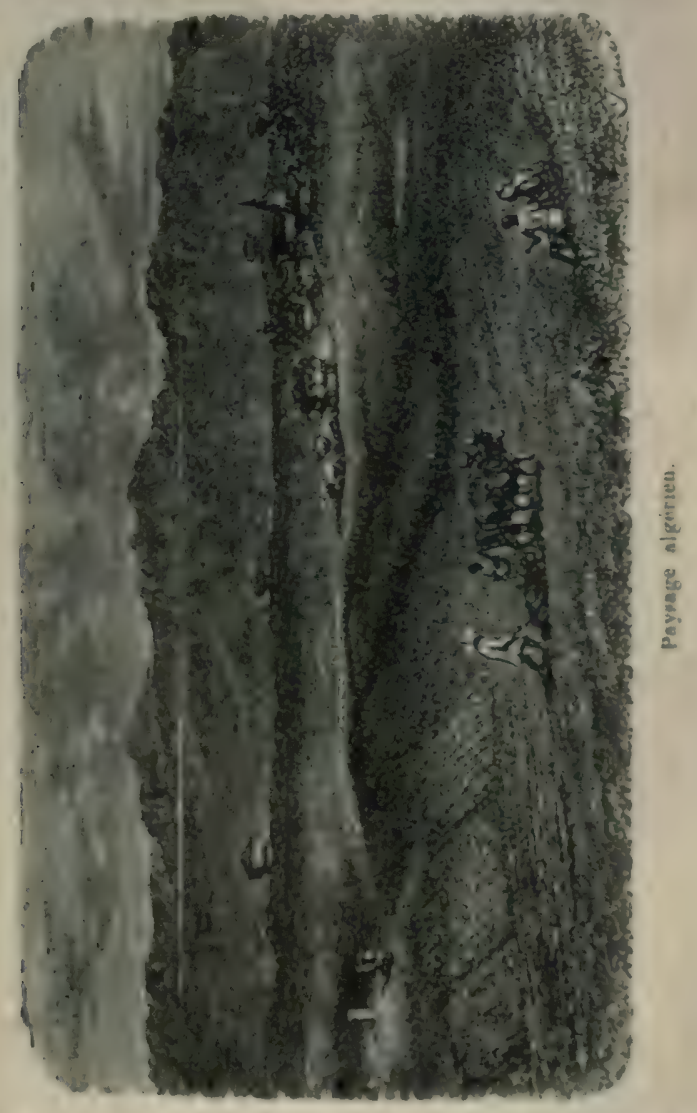

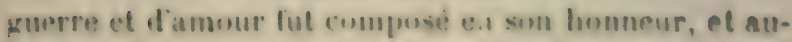
juurd hui encore il en reste des traces daus le's tribus nomades du Sud. 
Il est pénible d'ajouter que ce brillant épisode n'eut pour résultat que de retarder de quelques jours la prise de Ksar-el-Hirâne.

Le nombre des assiégeants était trop disproportionné, il s'augmentait tous les jours, tandis que le rarti d'El-Iladj-el-Arbi diminuait au contraire.

Finalement, Ksar-el-lliràne fut pris. Bon nombre de ses défenseurs perdirent la vie dans le dernier combat qui amena ret érénement. Si-el-Hadj-el-Arbi fit du nombre.

Avec lui finit le semblant de domination que l'émir Abd-el-Kader avait tenté d'établir dans le Sahra.

Mais ce qui survit dans lit mémoire des contemporains et qui se transmettra sans doute à celle des générations futures, c'est le souvenir de Messaouda-elllarzlia et de sun héroïque action.

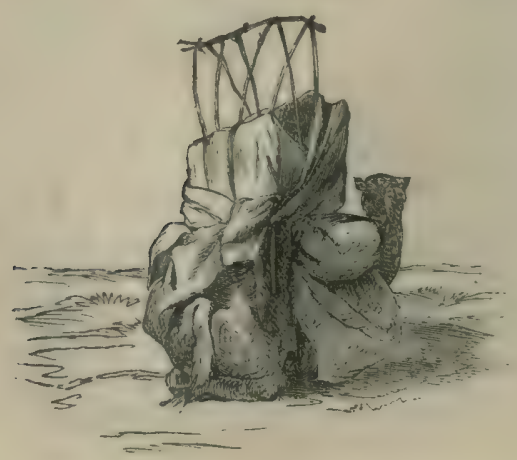




\section{LETTRE}

\section{5ะ 2 เ \\ REGGABS DU SUD}

Laghouat, le 5 juin 1858.

A M. le general DAUMAS.

\section{Mon Générat,}

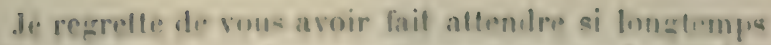
les quelyues renseignements que wous maser tait

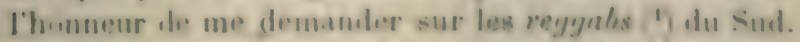

Jai, comme je vous lai somome tans ma dermere letlere, le type du genre chee las Oulat-Xavls: mallsemrensement mon pausre reggab est entre la vie et las mort depuis un mois de roulais tenir de sa bur.le le detail diss courses exlraurdinairos qu il a acenuplies:

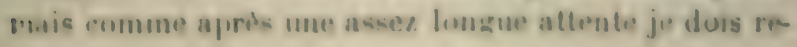

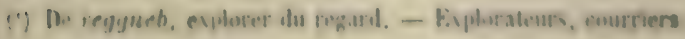


noncer à le voir en état de me raconter lui-méme ses prouesses, je vais vous dommer le récit de quelques-unes de ces dernières, parfaitement authentiques du reste et bien connues de tous les Arabes du cercle.

Ciest Ben-Saïdane que se nomme ce phénomène des coureurs.

Il est originaire de la tribu des Oulad-Saâd-ben-Salem, âgé de trente-huit à quarante ans: sa taille est grande, si conformation parfaite; ses jambes et ses pieds sont des modèles de vigueur et d'éligance, que la statuaire antique n'aurait pas reniés.

Ben-Saidane est toujours très simplement vêtu d'une chemise longue en colonnade et d'un burnous léger; une ceinture de cuir filali lui ceint les reins et sert à contenir quelques bouts de roseau dans lesquels il met ses provisions de bouche pour les grandes courses.

Il est chaussé de brodequins qu'il fabrique lui-mème avec du cuir de chameau et de la peau de chèrre. Il n'a pour arme que son fidèle bâton et un couteau à raser.

Vous savez, mon général, que les lrabes vicut du merveillenx dans les choses qui sortent un peu de la vie ordinaire. - Ben-Saïdane est donc considéré comme particulièrement doué par Dieu pour la marche, la faculté de ne jamais s'égarer et de vivre avec très peu de nourriture.

Voici en quelle circonstance ce don lui amrait étó accordé :

En 1845, Abd-el-Kader, prévoyant qu'il aurait à s'appuyer sur les Oulad-Nayls dans sa lutte avec nous, entretint, au moyen de Si-Chériff-ben-Lahrèche (notre khalifa actuel), des relations suivies avec les principaux 
personnages des Oulat-Si-1/umed et des Oulad-saitben-Salem.

Si-el-Boubali, chef de celte derniere tribu, ayant un a vis pre-n. a faire tenir a Abd-el-Kader, qui se trouvait alors vers Tiaret, lit chuix de: Ben-Saulane, quu flat ijeja en reputation comme marcheur, pour porter à l'émir une lettre pressée.

Ben-Sanlane ne conmaissait pas le pays de lOUn+-t quil avait a parcourir pour arriver a destination: il partit toutefois après sotre fait renseizuer sur la direection à suivre et la distance approximative. II rmfurta dans les roseaux de sa ceinture environ dis once: de rouina et suspendit a son cou une chibonen 's de lat contenance de trois litres d'ean.

II se mit en marche stimule par tous lin - ienthe et comble de caresses par son caid, qui lui dit : "Il u'y a que toi de capable daceomplir une parrille miscion. Pille est num seulement diflicile comme di-tance a parcourir tres rafuldement, mais encure enume danger fu-sible d'etre rencontre par les goums des colonnes, qui tiennent la campasme. -

Ben-Salilane, bien monce physiguement et morales ment, partit le matin du campement de sa tribu, qui

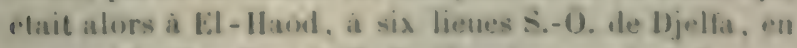
frenant la direction du X.-() . : il arriva sers trois heures dus soir a la hauleur de Sidi-Bomzili ; la, il sarréta un moment, consomma ensiron trois unces de remina :" el se remit en route.

Ia muit le surprit sur les hauls plateaux, il contumat à marefuer en so dirigenat sur les etudes: - molin le Indemain, vers huit heures du malin, il arrwait a

(1) Petile peau de boue.

(1) Parine de lats riki. 
Tagdempt, où se trouvait Abd-el-Kader, auquel il remit sa lettre. - Il avait parcouru cinquante-quatre lieuss en vingt-six heures.

L'émir et ceux qui l'entouraient avaient peine à croire all récit de Ben-Saidane; mais force leur fut de se rendre à l'évidence, en lisant la lettre, qui était datée, donnait des renseignements très récents et d'une grande importance.

Abd-el-Kader, voulant récompenser dignement le reggab des Oulad-Navis, dit à Ben-Saïdane: " Demandemui ce qui peut te faire plaisir, si cela est en mon punvoir je te le donnerai. " Ben-Saidane répondit: "U Prince des croyants, je ne te demande pas d'argent, tu combats pour la bonne cause, et c'est à nous ì t'aider de tous nos moyens; mais donne-moi ta bénidiction. Invoque Dieu pour moi, je me tiendrai porr bien récompensé.»"

- Ibd-el-Kader lui dit alors en lui imposant les mains:

"Que Dieu mette sa bénédiction sur tes jambes, et, par son aide, sois toujours ton propre cheval à toimème! ”

Il le congédia ensuite en le chargeant de missives pour les Oulad-Nayls.

Ben-Saïdane ne mit guère plus de temps pour revenir à son point de départ. "La bénédiction du marabout, raconte-t-il, - car il est parfaitement convaincu que elle a eu son efficacité, - avait produit son effet et je nis me sentais pas marcher."

Ciest depuis ce moment que nutre reggab a eu le, surnom de Aoud-Roho $\left(^{1}\right)$, sous lequel il est connu de tous les Arabes du Sud.

(') Cheval de lui-même. - Mot à mot, cheval de son àme. 
En 1819, Ben-Saidane et plusieurs Arahes de sa tribu allirent en caravane à Tugeurt pouracheter des dalles. Arrives dans cette ville, ils apprirent de Ben-Djellat, rue les goums de l'Est, les Bouizid. les selmia et leFilunls, setaient dirigós vers leurs campements qui -taient établis entre Mer-ad et le Djebel Bou-Khat, dans le but de les surprendre et de les r'azier.

Ben-Sandane soffrit pour aller immédiatement donner l'éveil aux Chlarl-sadid-ben-Salem, qui étaient loin d. soupconter cette agression.

Il partit done de Tuggurt sans presque se repuses. emportant seulement deux galottes ef une chibout.s pleine deau: il marcha jour et muit pendant quarantelum heures, sans prendre plus de tris repus deuvirun une heure rhacun; il arrisa it temps puor prevenir l.. suens, qui ser repliesent aussitit dans le Bon-Khat of eviterent la razia dont ils etaient menaces.

spres quelifues heures de repos, Ben-Saulaue, muni. comme in son depart de Tugurt, de deux galettes on de at pan de bouc pleine diau, se remit en mach. pour Tugeurt, wit il arriva cent deax hesures aprion on stre parti el avoir parcouru dans ce lape de tempo environ cenl quaranle lieues. - Il troura ses compagnomprets a partir; pour ne pas les retarder, il lit ses achats de dattes et revint avec erts.

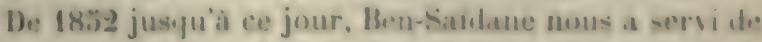
regrab; il a rendu dexcellents services a tous mos chef de colonne par la celerile de ses course-, sit comaissance parfaite du pays. -. Nus genéraux lont genereusement ricompense, et it est acluellement h Tabri dn besoin.

Mais Ben-Siblane nien exeree pas movins son melies fasure; rest, on preut le dore, par amour is la lowe. 
motion et sans doute pour maintenir sá réputation de coureur infatigable, à laquelle il tient beaucoup.

Pour s'entretenir les jambes, comme il le dit luimème, il vient assez sourent de Djelfa à Laghıouat en douze ou quatorze heures, faisant ainsi cent quinze kilomètres d'une traite.

Dans mes courses dans le Sud, Ben-Saïdane maccompagne toujours; il tient à honneur de marcher constamment à la tête des goums et de montrer le chemin.

C'est arec désespoir qu'il voit les cavaliers ou les: piétons prendre une autre route que celle qu'il trace, ou bien qu'il entend émettre un avis quelconque sur un itinéraire à suivre. - Il ne pardonnerait pas à son père, je crois, de dire un seul mot à ce sujet, tanl. son amourpropre de guide et de coureur est derenu susceptible.

Souvent, en chassant à courre la gazelle ou le lièrre, nous arons laissé Ben-Saïdane à deux ou trois lieues en arrière, mais toujours, moins d'une heure après, nous avons été rejoints par Aoud-Roho, qui ne manquait jamais, dans ces circonstances, en reprenant la t:te du goum, d'arborer au bout de son bàton un mouchoir en guise de drapeau et de l'agiter en cabriolant devant nous, comme pour narguer nos cheraux.

L'année dernière, au mois de juillet, pendant notre chasse à l'autruche, Ben-Saïdane fut plaisanté par les Mekhalifs, qui lui dirent: "Puisque tu te nommes Aoudhoho, pourquoi ne prends-tu pas comme nous des autruches à la course?"

Il leur répondit : "Vos chevaux sont des ânes! je les crèverais tous dans une longue course de fond, cela est connu des gens, et vous-mèmes ne l'ignorez pas; mais puisque vous me mettez au défi, je veux demain jaunir vos figures. Je partirai en même temps que vous au re- 


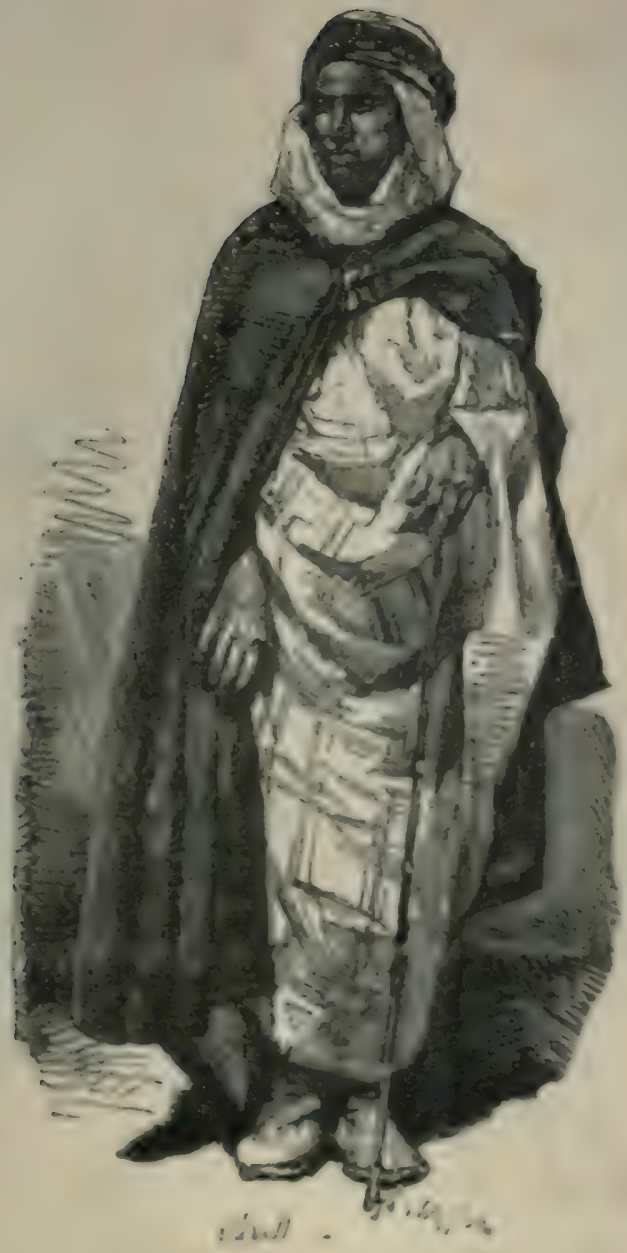

Deastalaxes 
lancer des autruches: puis, après avoir rejoint la premicre forcée, je veux ètre de retour au bivouac avant vous tous."

Le défi fut accepté. Le lendemain, Ben-Saïlane numaccompagna au Gâd (point d'où on relance les autruches). Aussitot que celles-ci parurent, nous nous élançâmes de toute la vitesse de nos chevaux à leur poursuite. Le reggab en fit autant arec ses jambes. Il arait résolu de suivre mes traces afin que je pusse constater son arrivée à la mort de l'autruche.

Ce jour-là, je forcai ma bèle en trente-deux minutes. après avoir parcouru seulement quinze ou seize kilo. mètres ( $\left.{ }^{1}\right)$.

Ben-Saïdane me rejoignit au moment où jachevais de dépouiller ma capture, opération qui avait dur trente-cinq minutes.

Il me dit: "Tu vois que je tiens ma parole et que ce's chiens de Mekhalifs, qui ne courent pas plus que leurs femmes, ont mauvaise grâce à s'attaquer à ton fils BenSaïdane. Je vais retourner maintenant au bivouac pour renverser toutes leurs marmites. J'aurai encore le temps de dormir comme un Kossri $\left({ }^{2}\right)$ avant leur rentrée. "

Il tint parole, et, deux heures après, je trouvai, quand je rejoignis notre camp, Ben-Saidane, arrivé longtemps avant tout le monde, se prélassant dans la tente du caïd des Mekhalifs, qui, pour le fêter en réconciliation, lui prodiguait son eau la plus fraîche et les meilleurs morceaux de hammoum.

Je pourrais multiplier à l'infini le récit des courses extraordinaires accomplies par Aoud-Roho; mais ce

(') II faisait très chaud; le thermomètre, à midi, marquait 50 degrés à l'onfure des betoums.

(z) Habitants des Ksours, que les Arabes traitent d'efféminés. 
qui pripiole el que je garantis exact sullit, je crui-

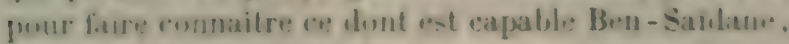

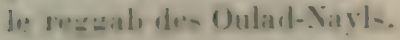

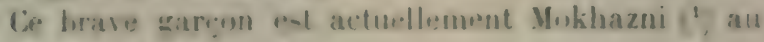
lourmatu arabe de Djellit.

L.or-püil sest agi de lincerire en cette qualité, solun sun disir, on lui a dit : "Achite-toi un cheval: tu sais ypun Vulihazni doil être montı.".

Ben-sablaur a malgreson resfrect et sa bonne tronte d.vant ses -mprivurs, ne put, a cette propusition, cunarver son serieux. Il se mit is rire de la facon la jula brusante et la phos cumique; contin, yuand il put farlere, il répondit au commamlant du puole :

" Peux-tu humilier aiusi ton serviteur en lui fropmsont de se servir d'un elorval? Be-l animal ma foi , - 11 .

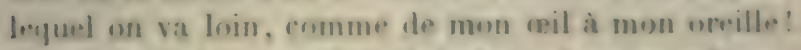
-. qui broit ef mange la substance qui nourrimat un. famille, -- qui henuit, lacise de graubles traces. - qqu. lon ne pent toujour-cacher farilement... Tu veus dume yuion rie de moi?... Nent-il pas, an contraire, de ton avantage davoir a ton sorvices un homme capable d. fare plus que tos meilleurs chevaux? qui n'aura jamas

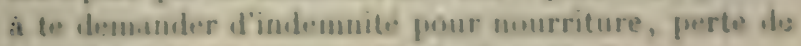
-heral, frais de ferrure, dentrellen, ete. ?..."

I de si lounmes rajoms il n'y avait rien a répumalre. Bon-Sundane fut inserit Khenal ${ }^{z}$, et placé, salon ses d.-irs, en tete de la liste de ces fonetionnanres, peur l.eipuels il a, sinon de la protié, du moins une grande commiseration.

1.es bons puetons ne sont fas ruros dans le Sahra, ot

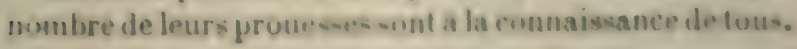

(') Cavalier solds.

(1) Caralive, homume de enteral. 
Je vais, mon généril, vous en riter quelques-unes. yui ont été accomplies dans ces dirniers temps et dont le souvenir est très vivant dans la mémoire des gens du pays.

Le nommé Ll-Touhami, originaire de Laghoual, sì il est encore, fut envoyé par le khalifa Ahmed-ben-Salem, en 1846, à Berryane, ville du Mzab. Parti à cinq heures du matin de Ksar-el-Hirâne, il arriva à destination le mème jour, à sept heures du soir, ayant parcouru la distance de trente-deux lieues.

Ce mème Touhami partit un jour de Nigouça, dans Ja mème année, et accomplit en vingt et une heures le Irajet de cette ville à Berriane (quarante-ciny lieues environ).

Pendant ces deux courses, il n’a mangé que quelques dattes et bu la valeur de deux litres d'eau.

En 1848, le nommé Maarouf-ben-Sliman, des Larbâ:, 'st venu de Guerrara à Ksar-el-Hirâne d'une seule traite, en marchant de minuit à sepl heures du soir; soit quarante-six lieues en dix-neuf heures.

Il est allé aussi en une journée de Guerrara à Ouargla.

El-Righi-Bel-Ouis, des Mekhalifs, en chassant l'autruche, fut entraîné à la poursuite d'un dólim, qui le mena plus loin que d'habitude et qui finit par lui échapper. Son cheval mourut au moment où sa dernière goutte d'eau s'épuisait. Il perdit la direction de ses compagnons pour revenir et s'égara.

Pendant trois fois vingt-quatre heures, il erra dans les plateaux, sans eau et sans nourriture. Il était très épuisé. Le jour il dormait sous un betoum, la nuit il marchait. Chez lui, on le croyait perdu.

Quand El-Righi arriva à ses tentes, on ne le reconnut pas, tant il était maigre et noir. Il raconta ensuite que 
co qui lawait smulomu dans sa détresse élait un rèves dans feqfuel sa more le soignait et lui dommait a buire a diserition: ce reve. qui se reprisenta plusienars foi-quen-

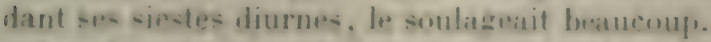

Le nommos Mhamed-lwn-Ilarzalliah, des Hadjadj.

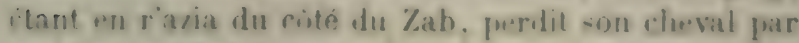
arovient: uldige de revonir a piend ver-at tribu, il dut mareher pendant puatre journeses de vingt-quatr. heoures. sans fuendre de nourriture ot sans boire. Cietait. il $\cdots t$ vrai, en hiser, et lit frmpuerature etait frobles.

Les Iumme Sidil-len-Sliman, de- Matmra, et qualre

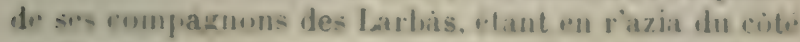
d. linerrarat Imtienent quatre jours sans maneer, mo

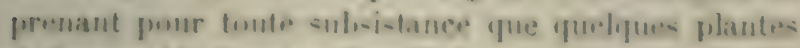

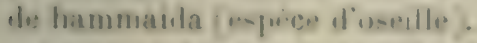

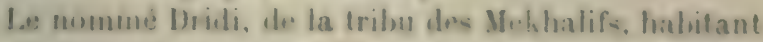

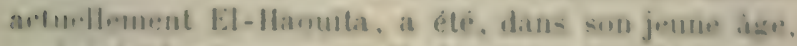
un intrepride chas-our. - In jour, étant tumbe sur un troupreaus de sefit moulluns à mam-lialles, il le pour-uivi dan- les kefs th of en tua six, en parreumant en sepl luevres un trajel te quinse lienes environ, dans un pays triss accidenté et dillicile.

fimprorte une fois par lardeur de la chasse, il suivil fermlant quatre jours les traces of un trouprau diautrumps. - Iu bunt le pe temps ayant muise sun ean it sers vives, il dut revenir chez lui, ne mangeant,

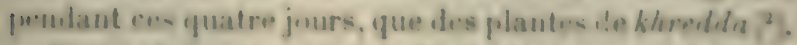

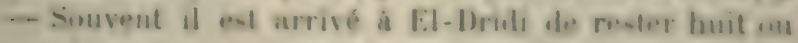

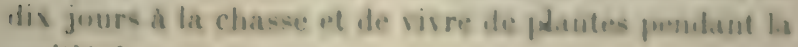
monitié de ce temps.

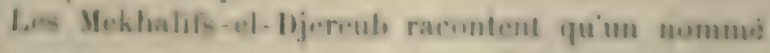

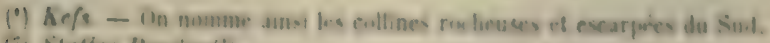

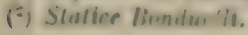


Messaoud-ben-Aissa, de leur tribu, mort il y a dix ans. forcait à pied des autruches au moment des plus grandes chaleurs.

Les exemples de longues marches accomplies rapidement et de sobriété exceptionnelle sont très nombreux dans le Sahra, on pourrait en faire un gros recueil.

Ces faits, qui nous paraissent si extraordinaires, n'étonnent personne ici; plus on avance dans le Sud, et moins le ventre, comme le disent les Arabes. domine l'àme.

Cheikh-Athman, des Touareg, qui était ces jours derniers à Laghouat, m'a cité quelques exemples qui confirment cette assertion. - Je dois dire que j'ai confiance dans la véracité de ce chef targui, pour l'avoir mis plusieurs fois à l'épreuve. Il ne m’a raconté, du reste, que des faits qui lui sont personnels,

Entre autres ceux-ci :

Dans l'année de l'hégire 1236, en été, Cheikh-Athman fit, avec un parti de soixante-dix Touareg d'Azeguer montés sur des mahris, une r'azia sur les Chambaàs de Ouargla.

A près avoir épuisé l'eau que contenaient leurs outres, ils restèrent cinquante-deux heures sans boire. Heureusement pour eux que leur r'azia rénssit: ils enlevèrent aux bergers des Chambaàs deux mille deux cents chameaux au moment où ils allaient mourir de soif.

Leur premier mouvement pour se désaltérer, aussitôt leur capture faite, fut de saigner soixante chamelles, dont ils recueillirent le sang et l'eau qu'elles avaient dans leurs estomacs.

C'est la ressource suprème, en cas de disette d'eau, que de tuer un chameau ou un mahri pour en boire 
Ie sans: mai- Il liut prentre la precaution, dit ChrikhAthman, de recueillir ce sang dans un rase, dattemelre ‘uil se suit congule; alons on jette le caillot qui si-t furmo., puis on lovit la partie liquide et séreuse yui m-te. Guand on na pas la patience dagir ain-i, le. sang que l'on buil chaud se fige sur l'e-tomar et lorùle les entrailles: il augmente alors le supplice de la soif.

Pendant six jours et demi, c'est-a-dire pendaut cent cimpluante-six heures avant la riazia, Cheikh-Ithman - des léns nont récu quave la valeur de six cents erammes de chatir de chamelle peour chacun.

Le chef des Tonareg d'Azeguer, Ikhenoukhen, en razia contre les Saud, avec quarante des siens montes sur dis mahris, est reste neuf jours et neuf nuits sans rau. Ils ont veru en buvant par quarante-huit heures un pen de sang ou deau, quils se procuraient en tuant leurs mahris. Lorsque to chiffre de ces derniers fut réduit a quinze, ils retournerent dans leur pays.

C"est un principue admis clrez les Touarog quiun parti en expeidition, à défaut de vivres et d'eau, peut, jour prolonger ses eperations, tuer deux

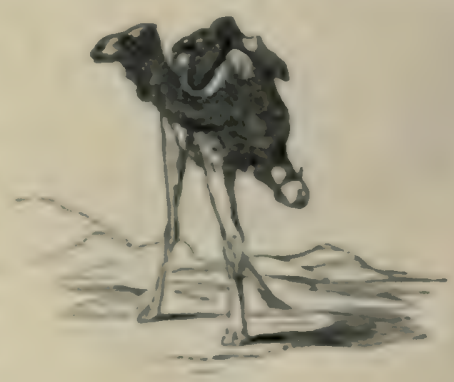

Mahri. malsris sur trois; mais jamnis plus, sous preine de preritre Ia vie.

tii malori. diment-ils, peut sauser trois hommors:

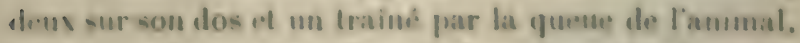
orise jamais qualer. Guand dome la riduction dess 
malıi- s'est faite jusqu'au tiers de leur nombre, le $\gamma^{\prime} e z o u\left({ }^{\prime}\right)$ rentre dans ses foyers pour recommencer une course plus heureuse.

L'année dernière, Cheikh-Athman, ayant entendı dire qüil était tombé de l'eau dans le pays, à six journées a l'ouest de leur campement, envoya un de ses serviteurs nommé Djouri à la découverte; puis, voulant s'assurer du fait par lui-mème, il partit un jour aprìs, avec quatre mahris. II suivit son serviteur à la piste pendant six jours et arriva en mime temps que lui à lindroit qui leur avait été clésigné.

Le trajet parcouru à pied par Djouri, en six jours, sans manger et sans boire autre chose que quelques gorgées do lait aigre, est d'environ cent cinq lieurs. C.e quil y a de plus remaryuible, c'est que cet imlividu, par suite de la morsure d'une vipère, n'a pluz que la moitié du pied gauche.

Enfin, Cheikh-Athman m'a allirmé qu'un de ses parents nommé Azoug, parti à la recherche de chameaux füris, était resté dix jours de vingt-quatre heures sans manger.

Azoug, monté sur un mahri, espérait toujours rejoinire les chameaux dont il suivait les traces. Il a raconté yue prndant les trois ou quatre premiers jours il avait suffert de la faim, mais que, s'étant toujours sanglé progressivement les entrailles, il avait fini par ne plus souffrir. Il était même dans une disposition d'esprit assez gaie; toutefois, il n'aurait pu accomplir un acte de grande vigueur.

Cheikh-Athman m'a raconté que les Oulad-Moulat, Irabes qui confinent aux Touareg-Hoggar, et dont le

(') Réunion armée en course de r’azia. 


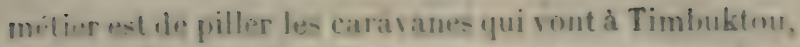
employaient un sinerulier muyen fuur conserver danleurs excursinns leur provision deau et the chair.

lls prennent de vieilles chamelles, qu ils privent d'ean pendant plusienurs jours; an mument de partiren course. its les font boires arfe exer-; rela fait, ils leur toupent I langue. puis les emminent arece eux.

1.nrsyue la provision deau et de vivres est épuises. Ios Mulad-Moulat turut steresaivement les whamellequi unt +u la langue coupere. lospuelles, parait-il, a cause de cette operation. ont conservé intacte l'eau absurbée au dipart. juur n'avoir pu la ramener dans le gosier, faute de langue.

Ces animaux leviement ainsi des réservoirs ambulants, dont la rhair est cunsommée par les hommes of par les rhevaux, qui sen muntrent tres avides. "6. chevaux, me disait throkh-Athman, hemnisesnt en vovant démpmer de la chatr, comme les votres quan.l ils voient l'orge.

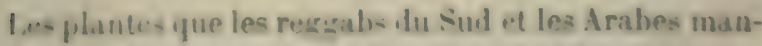
gont dans les muments de dieelte sunt celles-ci:

Komblat Statice Bundurlli.

Rl-Hammoumla, oseille sauvage.

lil-tiui-on, gine de ehicuré.

Br.zumb-el-Xindja pis de chamolle).

Talma, espresce de soursunere.

Rehia Danthomin Foskali.

A. ida, souches chroulrilloules.

Daghrmuns. Apterunthes gussoniana.

EI-Tifal, genre de laiterun.

Nelveg'se sedra, jujubes anuvages.

Danoun, Philiposa lusen et violarens. 
Aneb-el-Bctoum, grappes de pistachier sauvage térébinthe.

Cheikh-Athman m’a donné en outre quelques noms de plantes ou arbres, qui sont:

El-Yatil, arbre produisant des grappes jaunes.

El-Tolh, arbre à gomme et son fruit.

El-Harra.

Djerdjir.

El-Ghorran.

EI-Nefel.

Taouit, genre de graminćc.

Thafhif, id.

Drine ( $\left.{ }^{1}\right) ; \quad$ id.

En résumé, on peut dire que lorsque les indigines sont poussés par la faim, ils mangent à peu près tuntes les plantes, et ne font d'exception que pour celles reconnues vénéneuses.

(1) Le drine produit un petit grain comme du millet très menu et allongit.

Dans le pays des Touareg, des familles de fournis récoltent ce grain it en font des réserves assez. considérables. Les gens pauvres vont à sa rerinerclie et en font une sorte de farine, avec laquelle ils confectionnent des galettes et du kouskuus. Ceux qui vont ainsi s'approprier la subsistance iamassée à grande peine par la besogneuse fourmi ont soin de laisser quelques poignées de grains dans la fourmilière, afin que celle-ci puisse subsis-ter pendant quelques jours et reprenne l'espoir de nouveaux approvisionnements.

Nous agissons de même vis-à-vis des abeilles, quand nous recueillons les rayons de micl.

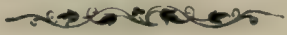




\title{
CANARDYILLE
}

f. $f$

\author{
LAPINBOLIRG
}

\section{CANARDVIILE}

Si co chapitre tumler janais putre less mains de cenx de mes conpragunns de chatse yui ont connu Canardville of Lappinluourg. je ne doute pas qu en se remémorant les jours heureux quils $y$ ont woru, ils ne semtent leur ecour baltre plus site et se rechauffor au souvenir

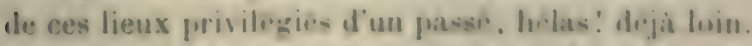

Pour moi, dusse je vivre cent ans, jaurai toujours prisentes a lie-prit les emotions juveniles, les junes untemose, que jai ressenties dans ies deux primcipales decousertes de ma vie de chassemer.

Puici ce quietail tanardsille, - je parlerai de Lapionlonurg ensuile:

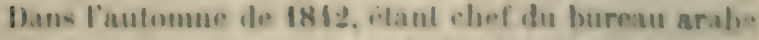

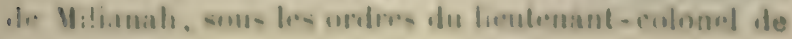


Saint-Arnaud, je fus chargé de la direction de petitrs opérations militaires qui avaient pour but d'achever la soumission de tribus ou de fractions restées hostiles.

C'est dans une excursion vers les plateaux du Sersou. pour rallier les derniers dissidents des Doui-Hasseni, que je décourris, à dix lieues au sud de Téniet-el-Hàd. près du confluent de l'Oued-Issa et du N'har-Ouassel ( ${ }^{1}$, un ravissant pays de chasse, unique dans son genre.

Il mérite une description toute particulière.

Le N'har-Ouassel, comme on sait, est le Chelif suprrieur. Il prend naissance sous Tiaret, se grossit à huit lieues plus bas des Sebaïn-Aïnun $\left(^{2}\right)$, traverse le pars

(') Fleuve naissant. Haut Chelif.

(2) Sebain-Aïoun, les soixante-dix sourees.

Une légende du parys veut que ce soit l'ancêtre du khalifa Sidi-el-Aribi qui ait, à l'instar de Noïse, fait surgir ces soixante-dix sources du sol.

Un jour, le khalifa fit le récit de ce miracle au maréchal Bugeaud, sans doute pour mieux glorifier son origine.

Il lui raconta longuement que le pays était à ce moment-là complètement privé d'eau, el que les populations demandèrent à son ancêtre Sidi-el-Aribı, qui vivait an neuvième siècle de l'hégire, de leur en procurer par sa baraka (bénédiction).

Sidi-ê-Aribi, touché de ces prières, se rendit à leur vou, et sur l'enplacement nù il se trouvait à cheval il fit faire à sà monture, en invoquant Dieu, soixante-dix sauts, et à chaque saut il surgit immédiatement une sumrce de l'endroit où portaient les sabots du cheval.

Quand le marabout crut avoir tiré assez d'eau de la terre, il se mit en marchr; les sources se réunirent et le suivment sous la forme d'une rivière à laquelle il donña le nom de N'har-Ouassel.

$1 \mathrm{innagina}$ alors de faire parcourir à cette rivière le plus de pays possible, afin que les musulmans pussent en profitur. C'est pourquoi, après l'avoir menée jusqu'aux monlagnes du Tittery, sidi-el-Aribi ramena le N'laar Ouassel, qui prit alors le nom de Chelif, jusqu'à la mer, et mit son embouchure pris de Mostaganem, presque sous le méridien de sa source.

Après avoir écouté paliemment cette lígende, le maréclial dit à notre kinalifa actuel : "C'est très bien, cela ; mais le Chelif, qui a une origine plıs ancienne que celle que tu lui attribues, était connu des Romains, déjà bien antérieurs à ton ancêtre. "

Le khalifa, d’abord un peu déconcerté, répondit : "C'est possible; mais alur's il n'avait pas d'eau. " 
mais mon imaginilim 11 aurait osi lo rever tel que je l'avais sous les yeux.

C'était un tourbillon, un fouillis d'oiseaux aquatiyurs. à faire croire que la gent emplumée du monde entir. s'était donné rendez-vous dans ce bienheureux marais. où sans doute elle avait à souhait, depuis des siècles, abondante pâture et quiétude.

Je fus vivement impressionné par cette merveilleuse découverte, et je me promis de revenir la compléter toutes les fois que je le pourrais.

J'étais loin encore de connaitre toute la richesse de? ce fortuné territoire de chasse. Ce n'est que plus tard, après l'avoir pratiqué plusieurs fois, que je découvris qu'en dehors de ces légions d'aquatiques, le pays était bourré de lièvres, de perdrix, et le plateau du Sersou, qui forme la berge droite du fleuve, peuplé de troupeaux de gazelles, de bandes d'outardes, de pluviers gris, de plwiers dorés, de gangas, de koudris $(1)$, etc.

Tout ce gibier, à l'exception des troupeaux de gazelles, n'avait jamais reçu un coup de fusil. Les Arabe: ne chassaient alors ni la plume ni le petit gibier. Que l'on imagine ce que furent nos premières chasses dans cette terre de promission.

Il nous fallait des mulets pour les charger. Mais n'anticipons pas.

Je ne pus revenir chasser à El-Goradia qu'à la lin de: 1844, quelques heures seulement; ce fut assez néanmoins pour m'assurer que le marais était aussi animé que l'année précédente.

En 1845, étant alors chef du bureau arabe de Ténietel-Hàd, je ralliai la colonne lu maréchal Bugeaud

(1) Ganga unibande, grosse espèce. 


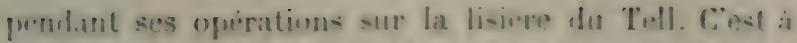

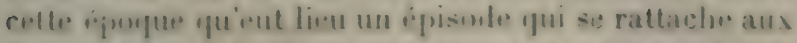

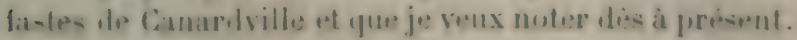

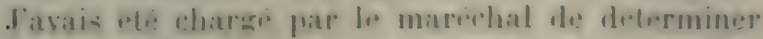

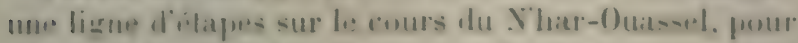
ume marrhe quil devait faire sur Bughar, en couvrant 1. Tell et en puursuivant Ibat-el-Kater, qui tenait alorJes hauls plateaux, diu il menaçait le Tillery.

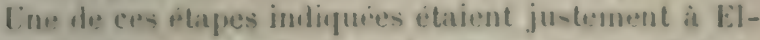
fiomalia, ou se trouvaient en abondance linu et le boinopessatres au spjour diune grosse colonne.

Javis mpingé au mariphal lit -urprise du grilorer: jo savais quil ny serait gas indilligent. comme chassemur émerite et excellent tireur.

fars dunc que je lui eus imliqur lemplacement du campr, sur un plateau sece of arire ou il devait sejourmere dems on trois jours pour allemire un convoi, je lui liremaryuer la grande quantite de canamls qui reltigeainnt autour de nous.

II en fut au-si en hanté que surpris.

"de vais etalulir le campl, me dit-11. el nums irons

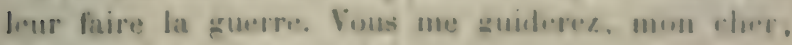
fuisfue rous comnaissery lo marais. a

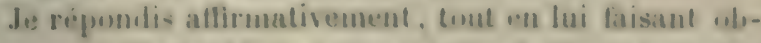

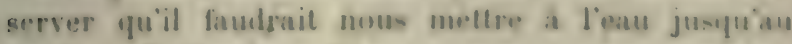

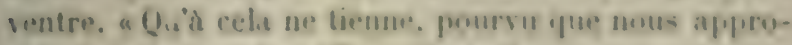

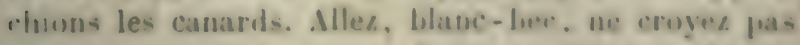
minfrayer avec botre eau. Je vous suivrai fartout? ajouta-t-il en souriant.

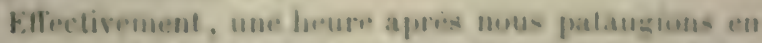
pleain marais. Jamais je niavais u l'exerllent marerthal si herureux. Il sectail, malere une temperature wenes

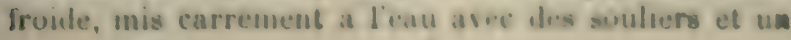


jantalon de troupier; il marchait et fusillait avec une ardeur juvénile; chaque coup abattait une pièce, tt souvent plusieurs, quand il tirait dans les bandes it. cols-verts et de sarcelles.

Deux heures après, nous étions à bout de munition: et avions notre charge de canards.

Le capitaine Rivet, alors officier d'ordonnance du maréchal, nous avait rejoints; il avait pris aussi sa part du gibier abattu.

Quand nous revinmes à la tente du maréchal, nous fûmes entourés par tout l'état-major et nombre de curieux qui vinrent admirer la chasse.

Le maréchal appelait à haute voix les retardataires: " Hé! Eynard, Pélissier, Trochu, venez donc voir notre récolte; - et il tàtait chaque pièce avec cette satisfaction de l'homme pratique, qui se retrouvait chez lui en toute occasion. - Regardez-moi cela! disait-il émerveillé, ce sont de véritables canards en caisse, des pelotes de graisse. " Et il les plumait un peu sur le dos pour démontrer l'exactitude de son assertion. "J'espère, chef, que vous allez en tirer bon parti. Ciest le cas d'inviter nos colonels. Quelle bonne idée Margueritte a eue de nous amener séjourner ici! C'est un double ravitiillement que nous allons y faire. "

En effet, les officiers eurent la permission de chasser, et le lendemain soir, toutes les broches étaient garnies; de nombreuses bourriches s'étalaient dans toutes les cuisines, à la grande joie des chef́s de popote.

"Fameux bivouac! bon pays tout de même ", répétaient nos soldats, qui avaient, eux aussi, trouvé dans la rivière de prodigieuses quantités de barbeaux, el sur ses bords des salades de cresson et de chicorée. Chaque gamelle avait son extra. 


\section{2.-otomis}

tom

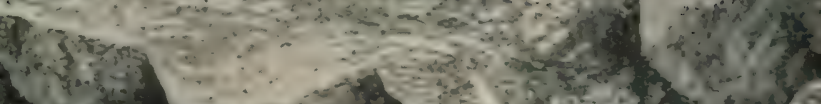
(1)

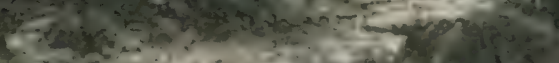

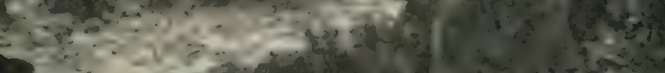

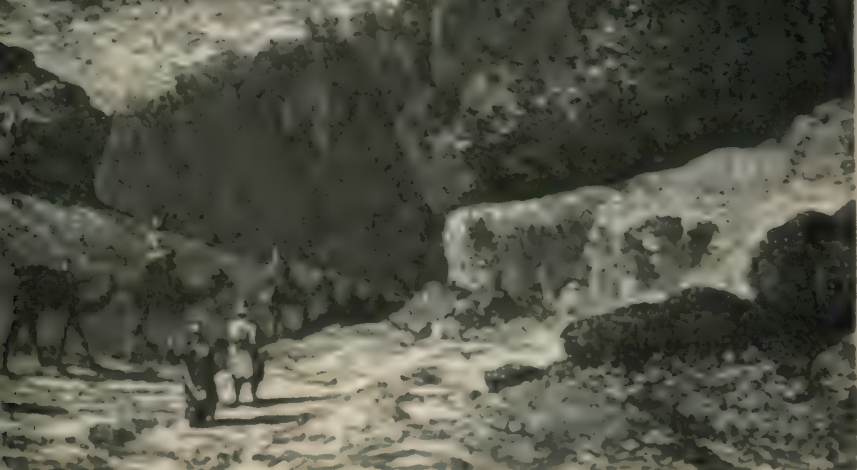

$+\infty$

$\cos +2 \tan x$

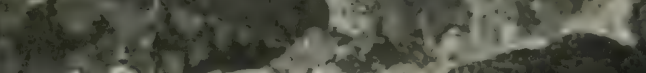

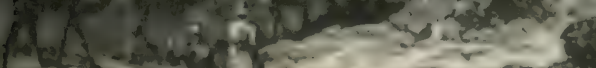



11 faut avoir vécu de la vie de nos braves soldats. dans aes longues expriditions faites dans un pays -ans re-sources, pour aroir une idée de la gaieté que donne La moin. Ire trouvaille propre à varier un peu le régime du biscuit et de la faible ration de viande maigre de tous les jour's.

Aussi ce fut avee des regrets unanimes et un excellent souvenir que la colonne quitla ce bon bivouac pour continuer sa laborieuse campagne.

A partir de celte année, je revins plusieurs fois tuus les hivers a la recha d'El-Goradia, avec ceux de mes compagnons de chasse qui en étaient aussi enthousiaste's qque moi.

Cirtait surtout lorsque la neige avait envahi Ténietel-Had pour des mois entiers, que nous éprouvions des joies a nulle autre pareilles a desiendre dans la vallie du Whar-Ouassel, ou, en raison d'une difference dialtitule de six à sept cents metres et d'une exposition au sud, nous trouvions une temperrature chaude relalivement et une prococe verdure.

Suts emportions alors motre outillage de campagne ef restions ales semaines entières, de la grande satisfaction des indigènes du cerele eux-mémes, qui préféraient venir traiter leurs alfaires dans un pays abordable, plutiot que diaflirnter les neiges de Teniet-el-Hád.

Ce n'est qu'en 1848 que jeas lidíe d'etablir a lilfioradia une construction qui, en nous servant de rendez-vous de chasee, nous dispenserait demporter a chaque excursion un matêrial de campenent quil atait jurlyuefois dillirile de sortir des neiges de motre pefite. Siberie.

Ce plan, une fois conçu, fut bientat mis à exestution. Janais alors pour commandant suprerieur un digne 
et brave officier, le capitaine Kennedy, du $2^{\text {me }}$ bataillon d'Afrique.

Il me donna quelques soldats de la garnison, et en huit jours, avec l'entrain exceptionnel que mettaient parfois au travail les joyeux zéphirs, j'édifiai CanardVILLE.

Je doute que jamais fondateur de ville ait eu autant de satisfaction que moi quand, au bout de la semaine, je pus contempler dans toute leur gloire mes trois constructions, formant les trois côtés d'un rectangle.

La première formait l'appartement des maitres et se composait d'une longue pièce de huit mètres de longueur sur quatre de largeur, avec porte, fenètre et grande cheminée. Sur le prolongement était la cuisine, d'une dimension moindre.

La seconde construction était destinée aux gens qui nous accompagnaient; elle pouvait loger une vingtaine de personnes.

Enfin la troisième était une écurie pouvant contenir une dizaine de chevaux.

Le tout bàti en pierre, crépi à la chaux, artistement couvert d'une toiture en tamarix et en jones de marais.

Voilà ce qu'était Canardville, situé à l'extrémité ouest du petit plateau qui domine le confluent de l'Oued-Issa (') et du N'har-Ouassel, à l'en l'roit où arait bivouaqué le maréchal Bugeaud.

Ce nom de ville des canards peut paraitre prétentieux, si l'on considère le nombre et la beauté des édifices; mais il était justifié sous le rapport de la population emplumée, qui, dans certains hivers, égalait celle d'une grande ville.

(") Rivière qui prend sa source près de Téniet-el-Hàd dans le DjebelR’ilass. 
Limasuration de lis mouredle cité ent lien quelques mois apres, en janvier 18 \$! a a moment le plus giboyeux de l'année.

La crémaillere fut pendur daus los régles.

Punr conte solennité et pour laire at Comaribille un: consiaration digne de son prosent et de son avenir. nuus résulumes, les six chasseurs que nous étions, le faire une guirlande de gihier autour du logis primcipal.

lo truisione jour au suir colle guirlamde etait achervie: les trente-deux mètres de développentent quiell. avait etairnt composés, sans la moindre ititerrupliun. d'un chafwetet de sazelles, lievres, outardes, canarils. grues, sarefles, vanneaux, pluviers, courlis, becassines, rilles, perctrix et cailles.

Cola faisait prés de trois cents pièces de gibier. dont la pulus grande partie fut expedice a nos amis et connaisances justupe sur la ciote, en flassant par Téniet. Milianah, Blidah, etc.

Cée-t ainsi que Canariville fut consacré et glurifie par tous les e-tomacs reconnaiscants.

En méme temps que nous fomdions la cite des canards, nous avions auss decide la criation diun juurnal des chasseurs.

liv devait ètre un repueil dans lequel on inscrirait. chatpues soir, le rroultat des chasses de la journece, les Gqusudes el fails interessants. Mais je dus dire que ce jumrnal we fut pas rolige trís exactement.

L." suir venu, on rentrait presugue toujours falıgué, surtout apress l'afriat, que nous ne manyuions jamais de faire, le suleil une fois rouche.

Un sait quit ce munembl les canards, qui se sont disjuerass dans la jumrnee pour aller chercher pafure au loin, revienuent coucher au marais. Nuus les altendior. 
dans les endraits les plus propices, et souvent nous faisions là, en moins d'une heure, le plus beau de notre chasse.

Les grues surtout ne pouvaient se tirer qu'à cette heure. Elles arrivaient par grandes bandes, en poussant leurs cris rauques et percants. Nous les abattions au moment où elles passaient sur nos têtes. J'entends encore le bruit qu'elles faisaient en tombant dans l'eau. Il fallait s'en garer afin de ne pas les recevoir sur la tète, car elles étaient assez lourdes pour assommer.

Quand donc nous rentrions le soir, vers sept heures, nous étions littéralement éreintés; nous ne songions qu'à nous sécher, à nous réconforter par un repas substantiel dont le gibier formait la base principale, et à prendre ensuite un repos nécessaire.

Les jours de plıie persistante étaient consacrés à la lecture de bons livres que nous emportions; la journée alors se passait à se bien chauffer autour de la grande cheminée de notre unique salle, à lire et à écouter les histoires des différents personnages qui renaient nous voir et humer notre café.

Quelles bonnes journées nous passions ainsi, chassant à outrance ou nous reposant de mème!

Notre commandant supérieur avait un caractère si sympathique, des qualités de cœur si élevées, un esprit si bienveillant, que l'on aimait à vivre près de lui.

Il avait traversé la vie en philosophe, prenant toutes choses par leur meilleur côté. Il avait beaucoup vu et beaucoup lu; sa mémoire était ornée d'un bon choix de faits et d'observations intéressantes dont nous profitions tous, quand il racontait et causait avec nous à cour ouvert.

Jamais je n'oublierai cet excellent homme, qui a eu 
la rare bonne fortume de nivoir, dans le cours de -on existence. que des amis ou des appréciatmurs bienveillant:, et, laus sa plus large acception, une roputation meritie dheusute homme.

Je molais particulièrement allaché à lui a la suite d'un accident dont $j$ avais ete la cause indirecte.

Lors diun retour de congé que jetais alle praseer en France, Kenuedy biait venu a ma rencontre. a quelyues kilometres de Triniet - el-Had, avec nus camaraders et bun nombre de cavaliers arabes qui roulurent alsoluuent, pun nous faire honneur, bruler de la poudre dans une fantasia.

On sait qu'il n'e-t pas toujours facile dempucher cers diables al'hommes de se liver a leur exercice favori ':

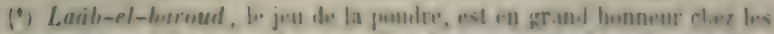

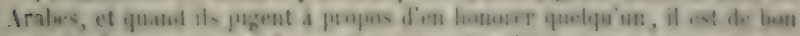

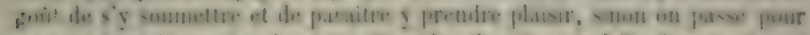

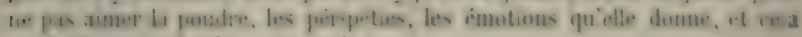

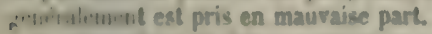

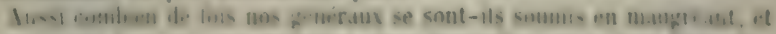

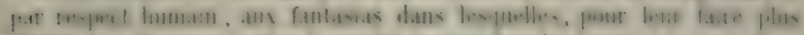

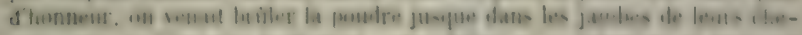

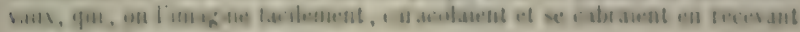
Cot ene ns des fimaters arales.

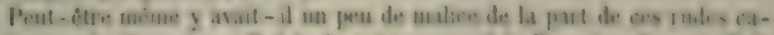

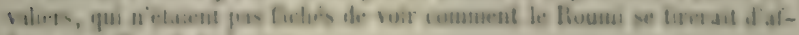
Date an milima de ia batione.

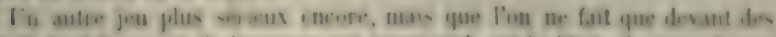

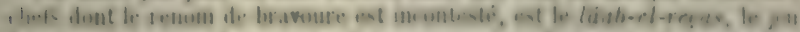

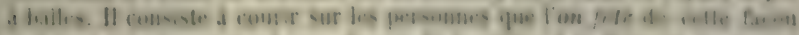

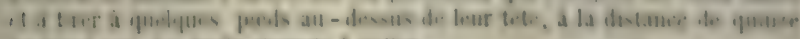

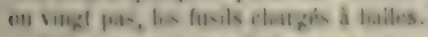

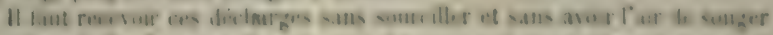

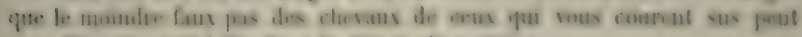

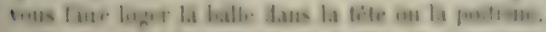

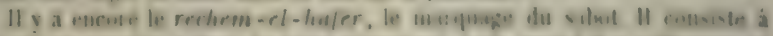

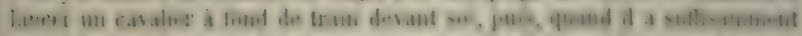

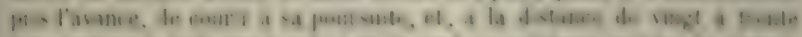

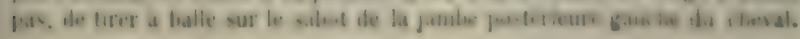


le jeu de la poudre se fit done sur la route même oì nous marchions.

Les cavaliers partaient deux à deux d'une centaine de pas, arrivaient bride abattue, et tiraient leurs coups de fusil à quinze pas de nous, puis arrètaient leurs chevaux court, et faisaient demi-tour pour laisser la place à d'autres.

Ce jeu ne nous amusait nullement, il était assez dangereux; la route était étroite, coupée à pic à gauche et en talus très raide à droite. Nous avions, du reste, à nous raconter des choses intéressantes, que tout ce mouvement nous empêchait de dire. Nous fìmes donc une injonction plus formelle que les précédentes, et la fantasia prit fin. Mais nous avions compté sans deux fanatiques, qui, ayant leurs fusils chargés, voulurent bruller leurs derniers grains de poudre.

Comme les autres, ils vinrent vers nous à fond de train, déchargèrent leurs armes, et arrètèrent leurchevaux. Toutefois, l'un d'eux ne put le faire complitement. Ses rènes de bride se rompirent dans la saccarle d'arrêt. Son cheval, ne se sentant plus retenu, s'élanca en avant de nouveau avec tant de vigueur. qu il vint heurter le poitrail du cheval de notre paurrt commandant supérieur, qui, n'ayant pu se garer i temps, fut culbuté dans un choc terrible.

Il faut alors que la balle frappe le sol au moment où le pied du cheval vient de le quitter.

Tous res jeux, auxquels jai souvent pris part dans mon jeune temps, tombent en désuétude.

Il ne faut pas le regretter : des mours et des allures plus douces remplaceront ces dangereux exercices, qui avaient pour but de former la jeunesse au mépris du danger.

Mais il n'est pas lors de propos de constater qu'ils ont été en grand honneur chez les Arabes de la génération actuelle, et de dire qu'ils s'en suuviennent elıcore. 


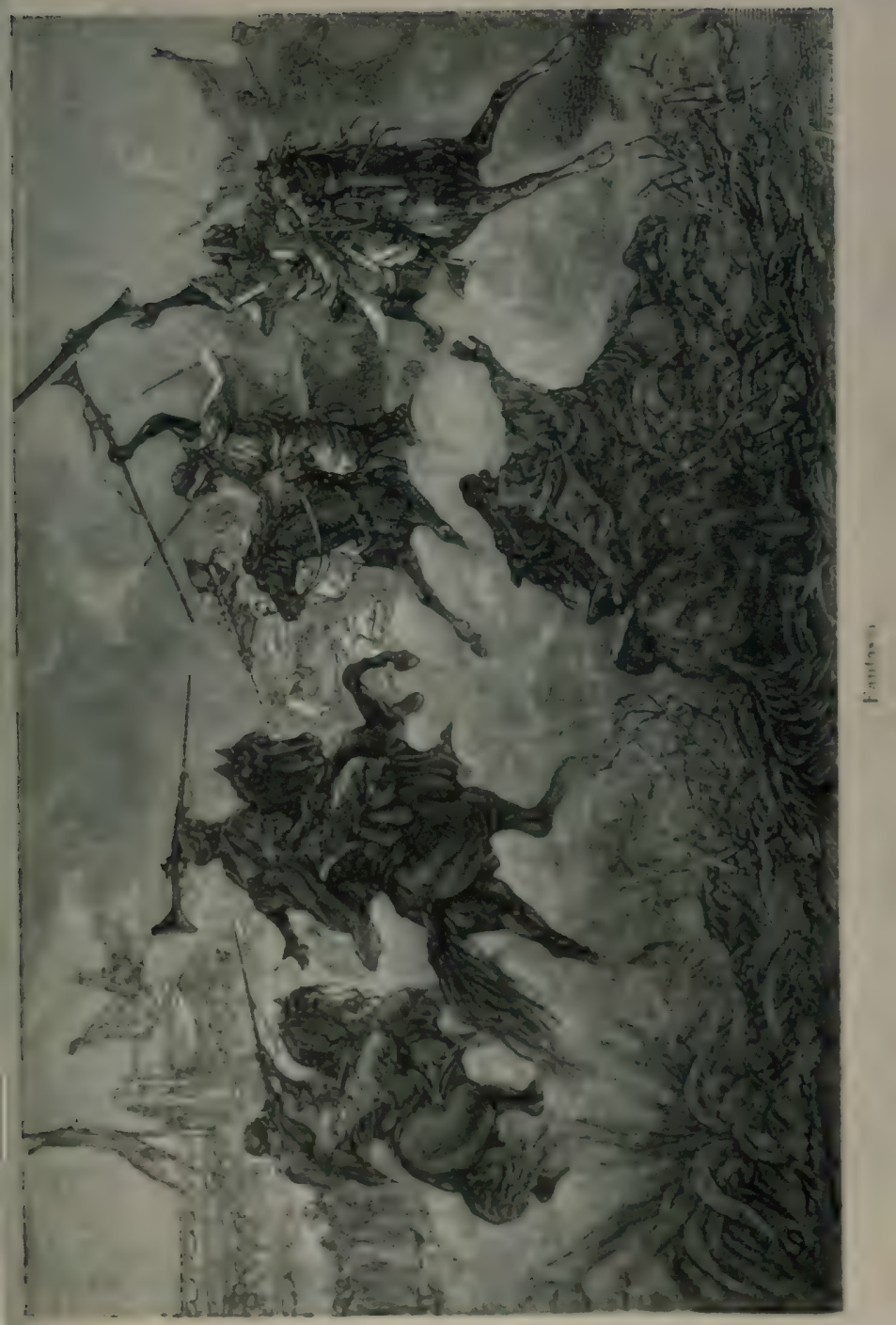



Je me jetai i lore prour le relever : il avait perdu connaisance. Quand il revint i lui preu apress, nous uous aperômes qu'il avait l'épraule gauche démise... Malgré cela, il voulut quion le remit à cheval, pour ne pas assumbrir, par l'aphareil d'un brancard, notre rentrée dans Téniet.

Le cavalier, cause de l'accirlent, était dans la consternation et daus lapprébension d'un châtiment qu il juent avoir mérité par sa lésoböissance. Kunnedy. maloré ses souffrances, prit à tache de le rassurer luimime et de lui dire yu'il ne lui conservait pas rancune.

Rentrés au camp, il fallut procidor a loperation de la remise de l'epaule; on chloroforma notre patient, sur le liras duypel plusieurs hommes furent appeles a tirer. Soit que lioperation fot plus ou moins hien faite. soit quw. la violence du choe, en débuitant la teite de Thumerus, rit lose une partie essentielle, la muirisun se) fit mal, et une sorte dankylose empecha le jeu de l'épaule.

Rien n'y fit : ni les eaux de Baréges, ni leo terpinges les plus energigues. I e bras conserva de la raideur.

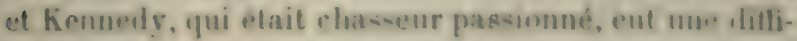
culte tullement grande a mettre son fusil en juthe, qu il dut remuseer it lien tirer.

Malere celte calamite, éstait lui qui praraisait prenilre le plus gatement sette situation. Guand il m'en royait altriste, il me disait : o Mon rher anni, Il fmurait marriver folus mal. Il me reste bon pied, bon wil. Si je ne puis abattre autant de gihier quiautrefors, je marcherai tout autant, et prendrai plat-ir a vous voir faire. II faut de la phtilosophie en ce monele. = Coeba itait simplement dit, comme it te perrait. 
Non seulement il ne voulut pas qu une punition quelconque fût infligée à celui qui l'avait estropié, mais plus tard il lui fut en aide dans plusieurs circonstances. Aussi était-il vénéré par les Arabes du cercle, qui le connaissaient et disaient de lui : kalbou kebir, son cœur est grand.

Je n'aurais pas trouvé ma description de Canardville complète, si je n'avais consacré quelques pages à celui qui était pour moitié dans sa fondation, et qui my tint si bonne et affectueuse compagnie.

Dans les années suivantes, Canardville devint une base d'opération pour des chasses plus éloignées, qu'il nous prit fantaisie de faire, tant pour varier que pour suivre nos bandes d'aquatiques, qui, à force d'ètre persécutées dans la Recha, allèrent chercher dans le Sersou des lieux plus hospitaliers.

Nous fìmes alors de petites expéditions, soit en remontant le cours du N'har-Ouassel jusque dans le pays des Beni-Lint, où se trouvaient des petits lacs couverts de bandes de courlis, soit en allant sur l'Oued-Fdoul, à Tzarritz, à Susellem, cours d'eau du Sersou central, soit enfin en allant sur l'Oued-Ourq et à Taguine, où nous retrouvions la mème abondance de sauvagine qu'au marais de Canardville lors de ses preniers temps.

Ces grandes parties étaient surtout séduisantes frar les chasses à courre la gazelle, que nous faisions arec les meilleurs cavaliers du cercle, qui se ralliaient $\dot{a}$ nous pour ces sortes d'excursions.

Je dois parler ici d'un marabout grand chasseur, qui obtint quelque célébrité dans les cercles de Ténietel-Hàd et de Boghar lorsque je l'eus fait comnaitre. 


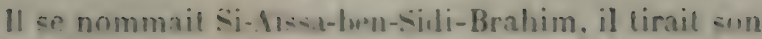
orisime diune famille de maralouts de Besusaarla. Depuis yutlques anness il s'olait fixé chez les Bou-liclse, ail il avait trouvi un refuge contre des haines et des perserintions de partis.

Si-Al-sit po-sfilait une mumbreuse famille: il vivait, lui et les sims, de chasse et de dons pieux que lui faisairnt ses Réperas ${ }^{2}$, sans doute le regime qui ré-

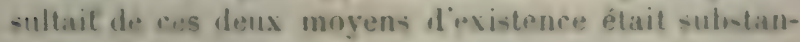
liel, war notre marabout a vait une bonne firure, large, colures. de grands yeux bleus, de mamifiegues dents blam.lses, une taille solide. deg membres vitworeux et un air avenant qui amenait cefte reflexinn: Visci un juyeux complere.

In jour que je chassais la gazelle vers les limites

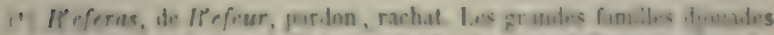

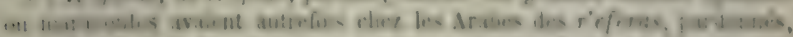

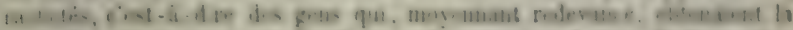

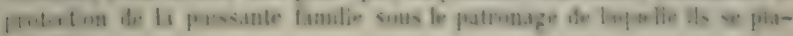
caine

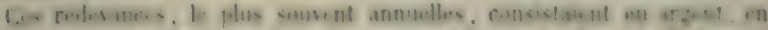

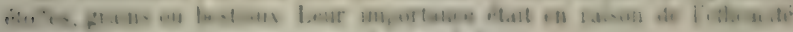

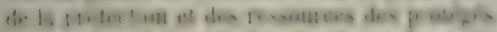

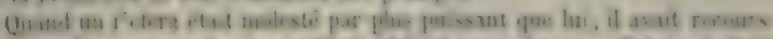

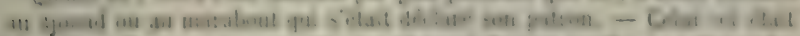

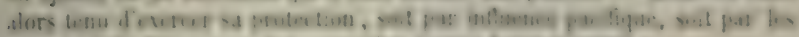

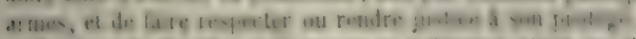

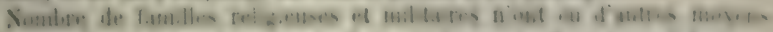

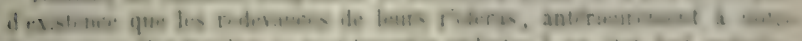

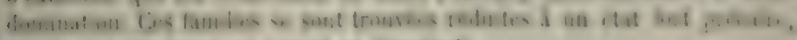

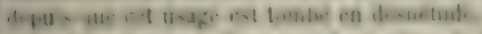

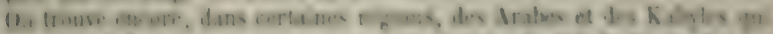

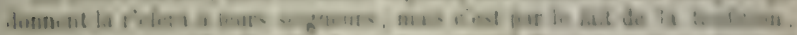

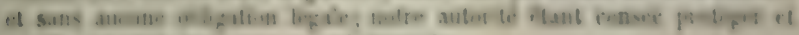

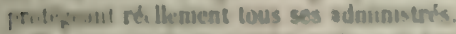

C.

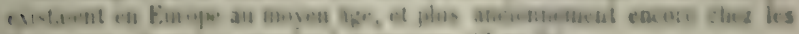

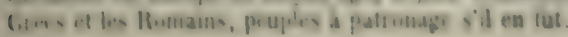


du pays des Doui-Hasseni, sur lOued-Fdoul, je fis la rencontre de Si-Aïssa. Je n'avais pas été heureux : les gazelles, au lieu d'ètre en troupeaux $\left({ }^{1}\right)$, étaient dispersées et clair-semées. C'était déjà le printemps, époque où chaque couple s'en va pour son compte.

J'avais dit mon désappointement à Si-Aïssa, qui, me connaissant de réputation, s'était mis à marcher près de moi pour me renseigner sur une région appclée El-Coudiat, où il y avait toujours beaucoup de gibier.

"C'est inutile, me dit-il, de chercher des troupeaux, ce n'est plus la saison, ils se sont dispersés; mais si tu veux, je te ferai tuer autant de gazelles que tu roudras, par mon moyen à moi.

- Je ne demande pas mieux, lui dis-je, et quel que soit ton moyen, il est le bienvenu. Que faut-il faire?

- Oh! c'est facile. Tu vas mettre pied à terre et te sacher derrière ce buisson de jujubiers; moi, j'irai chercher ces deux gazelles que tu vois là-bas, - et il montrait effectivement deux gazelles qui étaient à environ six cents mètres de nous, - je te les amènerai là, tout près, ajouta-l-il; je les ferai passer à cette touffe de cheihh. »Et il m'indiquait un bouquet d'armoise à quinze pas du buisson devant lequel nous étions arrêtés.

Je regardai mon homme bien en face, pour m'assurer qu'il parlait sérieusement. Lui, devinant ma pen-

(') En automne et en hiver, les gazelles se réunissent en troupeaux. J'en ai vu qui avaient jusqu'à deux ou trois cents têtes.

Plus un troupeau est nombreux et plus facilement on l'approche, pare: que les gazelles, en se massant, se gênent entre elles pour courir. Ell': sont quelquefois tellement rapprochées les unes des autres, qu'on entend leurs cornes s'entrechoquer, ce qui produit comme un roulement de coups de bagueltes. Dans le printemps et l'été, elles se dispersent pour la repruduction. 


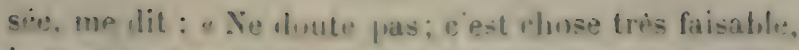
le temps est jropuce, arrange-toi seulement pour nètre pits vu: mais tu pourras regarder a travers le buisson et te libennuyer a me voir conduire les gazelles. Te tes presse pas surtıut, tu les tireras anssi près que tu voudras.

- Va, lui dis-je." Et, tris impattent de voir le résultat de ses promesses, je m'installai de façon a ctre parfaitement masugue par mon buisson.

Si-.li-at sen fut au petit trot, emmenant avec lui l. cavalier qui conduisait mon cheval. It prit a gaurhe. laissant les deux gazelles à sa droite, comme sil ne s'en occupait pas. Parvenu à envirun douze cents mitres, il oblinga a droite, mit les deux gazelles entre lui et moi, puis s'en rapprocha alors en marchant au pas. Cilles-ci, in mesure que Si-.1ssa remait vers elles. se mirent en mouvement, a une pelite allure; nietant pas trup pouscees, elles trottinatent, marrhaient, sidrrêtitent, allaient dans un sens, pruis dans l'autre, excepté. hio:n entendu, du côté du rabalerear.

Colui-ci, selon que les guzelles inclinaient trop at droite on a gaurfure de la ligne qu'il voulait leur faire suive. faisait des clangements de direction, sednismait méme, pour leur donner plus de confiance, et reprenait son manege.

Cost ainsi que, far dos mourements quil commu-

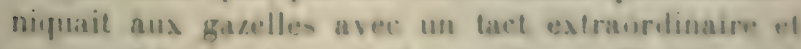
une parfaste intuition de leurs allures, Si-Aissa l. amena la ou it mavait dit quil les ferait paserer.

Javais suivi toute celte scine de raballage asee un vif plaisir. Je tuai le mále du couple, qui vint se plarm exartement prés de la touffe dirmose indiquee et is arréta, conme prour me dunner le tempes de le linen riser. 
J'étais tollement ébahi de ce que je renais de vorr, 'que je ne songeai pas à doubler sur sa compagne, qui s'enfuyait à petite portée.

Aussitot mon coup de fusil tiré, Si-Aïssa était acrouru au galop. "Eh bien, tu vois, me dit-il, que ma manière est bonne. On n'éreinte ni soi ni son cheval, "t on tue autant qu'en courant les troupeaux."

Javouai que cela était exact, mais que lui seul me paraissait posséder l'art de faire jasser les gazelles là tù il voulait. "Oui, moi et mes deux fils, me dit-il. Nous nous sommes exercés tant de fois, que nous faisons marcher à notre volonté la plupart du gibier qui piète. Si tu veux tirer des outardes, je t'en amènerai.

" - Comment, des outardes (1) aussi?

"- Oui, elles sont mème plus faciles à diriger que les gazelles, elles n'ont pas la tète si dure. "

Ce jour-là nous ne pûmes en trouver; mais le lendemain, Si-Aïssa, que j'avais emmené coucher à Canardville, me conduisit dans une région où il en connaissait, et m'en fit tuer trois dans la matinée, de la même façon qu'il m'avait fait tuer la gazelle de la veille.

A partir de cette époque, avril 18.49, Si-Aïssa fut de toutes les parties dans le Sersou; il s'était intitulé mon rabatteur en chef, titre que personne ne songea à lui contester.

Nous l'avions surnommé Bas-de-Cuir, parce qu'il portait des bottes de maroquin, et surtout parce que nous nous plaisions à lui trouver certains points de ressemblance avec le célèbre Bas-de-Cuir du roman des Pionniers.

Bas-de-Cuir donc m'a fait tuer autant de gazelles ef

(') L'outarde huppée d'Afrique, genre Houbara. 
dimtarites que jai voulu. Je miatais passiunné puur

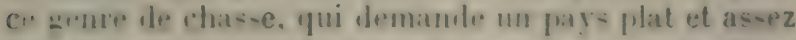
d.enuvert pour yue laetion du rabulteur sur le gibier mit rom-tante, jusyuau mument nit il est amenr at portée du tireur.

I.es mouvenents divers que l'un voit faire anx loetes yui se rapprochrut insensiblement du ehaś.ur qui les gurefte interessent autant que le rísultat final.

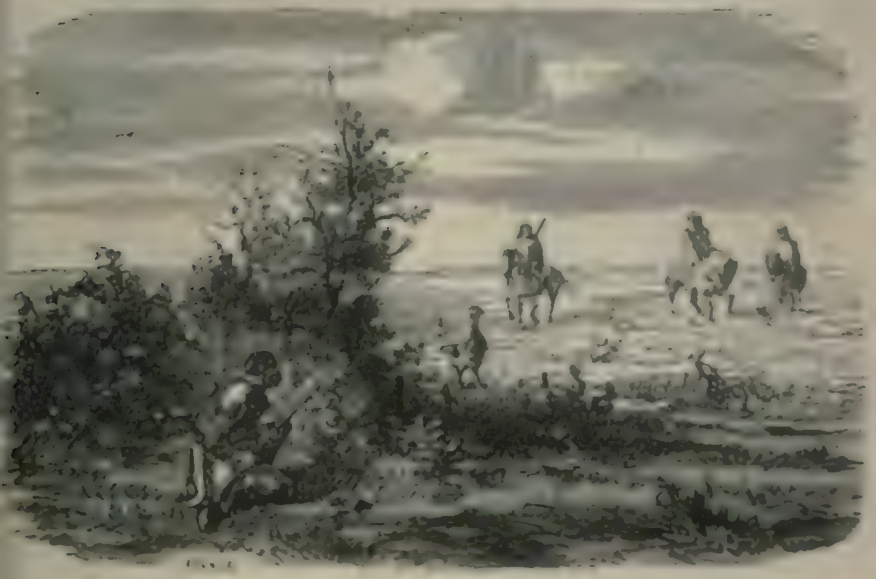

Rabaluge der outanies par st-Aisa of ses tin.

Si-Aissa avait, comme tous les hummes qui ont menes la vie imlipendante des sulituiles. une srame apulude prour se suftire a lui-mene. Il clait industrieus au fussible, et trouvait a se sustenter là wh i iautres seruient morts de faim.

II connaissait a fond les resouroes du farys. des glateaus quil avait tant de lisis parcuurus; il saviot trouver de linu lorspuil n'y eu avat pas dappare.ole 
à la surface du sol. Il était grand dénicheur de nids de toutes espèces d'oiseaux, savait récolter, dans la saison, les terfès ( $\left.{ }^{1}\right)$, les champignons, les jujubes, les grappes du pistachier sauvage, les tiges d'alfa, les différentes plantes plus ou moins nutritives. Il savait tendre les pièges et y prendre tous les animaux pour lesquels il ne voulait pas dépenser sa poudre. De plus, il n'avait pas son pareil pour découvrir le gibier au gite.

Bas-de-Cuir avait toujours dans sa djebira du sel et des épices, qui servaient à assaisonner ses repas improvisés. Jamais je n'ai mangé de meilleures grillades que celles quil nous faisait avec des cotes de gazelle, et le foie qu'il préparait en melfouf $\left({ }^{2}\right)$.

Un jour que nous avions égaré notre déjeuner et que la faim nous talonnait, Si-Aïssa me dit:

"Si tu veux que nous mangions, c'est facile. " Tout était facile avec lui.

- Tu as donc quelque chose dans ta djebira?

- Non, je n'y ai que du sel et du piment.

(') Truffes blanches du Sersou. Ce tubercule, que l'on trouve en grande quantité dans les plateaux du Sud, dans les bonnes années, a la forme de la truffe; son goùt est particulier et semble tenir du clamapignon et de la ponme de terre. Il y en a deux qualités : les terfès rouges et les blanes. Les rouges sont plus estines.s. Les Arabes en sont très friands; ils les mangent en ragoût avec de la viande, cuits avec du lait, ou simplement sous la cendre. C'est de cette façon qu'ils ont le plus de parfum et de saveur.

(*) Melfouf, de meleffef, voilé, 'entouré. Voici comment se fait celte préparation, aussi simple qu'excellente :

On fait griller sur la braise le foie de la gazelle. Lorsqu'il a une demicuisson, on le retire pour le couper par morceaux de la grosseur d'une noix. Ces morceaux sont saupoudrés de sel, puis entourés de panne ou toilette de la gazelle. On les enfile ensuite en brochettes et on soumet cellesci à la cuisson finale. Quand elles sont bien dorées, el que la succulenle graisse a pénétré les pores du foie, on retire. On mange brùlant, et, sellon l'expression de Brillat-Savarin, on voit merveille.

Les Arabes, qui sont parlaits rôtisseurs, ne manquent jamais de faire du milfouf tontes les fois qu'ils font rôtir leurs moutuns. 
- Ce nie-t pas assm lui dis-je.

- Ciest vai; mais $-i$ tu veux te rontenter d'un livire rbli, de bunnes figues et d'un bon coup d'eau fraiche. jo vais te procurer tout cela.

- Parilieu, uui, je m’en contenterai, et le plus tùt sera le mieux.

Yous chassions ce jour-la du côte de Kef-Recheiga. nous étions à la recherche d'antilopes bubale-, qui nous avaient eté signatees dans cette region, mais que nous navions pas encore rencontrées. Bas-de-Cuir, evidemment satisfait de montrer une fois de plus sun savoir-faire, prit la direction des collines, dont nous etions peu éloignés. Chemin faisant, il mit du putumb dans sun fusil a la place des balles, puis, sans ralentir lallure, il regarda altentivement autour de lui.

Nous a asions gats fait un demi-kilomidre dans notre numelle direction, lorsque je le vis arriter son cheval et viser une touffe de semnar' $(\$)$. Je me dontai hien que cétait notre dejeuner qui commençait a prindre. fillectivement, te couj, is peine parti. je vis rouler un beau lievere que Bas-de-Cuir se mit à saiguer et a depouiller en un tour de main. "La contree est bonne, me dit-il : il est tries gras. Allons a l'vau a present." - El, d'un temps de trat de ciny minutes, it me conduivit sur le bord d'un torrent pierreux, que je trouvai complïtement à sece, selon mes prèvistons.

Jen lis la remarque, mais Bas-ile-Cuir, qui avait dója ntlache son cheval et ramasse des branches seches auxiguelles il avait mis le feu, me fit voir une grabule pierre plate dans le lit du torrent, il la sombleva avec une vigneur preu commune, et décourrit une excava-

(1) Sirte de faus alfa, Stipus tenucissima. 
tion creusée dans le roc, de la contenance de deux hectolitres environ, à moitié pleine d'une eau potable et fraiche. "Voilà ma citerne, me dit-il; c'est une cachette qu'un vieux pâtre des Shari m'a montrée, un jour que je partageai avec lui une gazelle que je venais de tuer. " - Et, tout en me racontant cette histoire, il embrochail le lièvre avec une branche de jujubier, le saupoudrait de sel, d'une pincée de piment, et le soumettait à l'action du feu.

Comme je lui faisais compliment de sa lextérité, il me dit: "Mais il manque encore les figues. Tiens, prends la broche; pendant que le rôti achèrera de cuire, j'irai les chercher. Je dois en trouver; c'est la deuxième saison, les pâtré n'ont pu les manger, puisqu'ils ne sont pas venus dans ce pays depuis le printemps. "

Et il s'en fut dans la direction de quelques anfractuosités rocheuses qui nous dominaient à deux ou trois cents mètres. Un quart d'heure après il revint avec la moitié de son mdol $\left(^{1}\right)$ rempli d'excellentes petites figues, mûres à point, cueillies sur des sauvageons, qui avaient poussé là de quelques graines sans doute transportées par des oiseaux nourris de figues des jardins de Goudjila ou de Ben-Hammad.

Quoi qu'il en fût de leur provenance, que je ne cherchai pas trop à approfondir sur le moment, nous les trouvâmes parfaites et le lièvre très réussi. Il n'est tel qu'un bon appétit pour assaisonner les mets.

Cet excellent repas terminé, nous bûmes à mème à la citerne de Bas-de-Guir, que nous recouvrimes avec

(1) Mdol, qui ombrnge. Nom donné à ces grands chapeaux à larges bords et de hautes formes coniques, que I. s Arabes portent en été pour se préserver du sileil. 


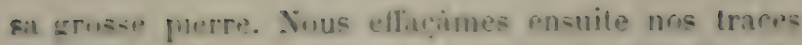
dan-ses alentours, puis nous primes la direction de mos tentes. yui avaient été plantées ce junr-la a la sturce de Rass-Fdoul, remellant au lendemain la recherelor des antilopes bulnales.

Quello ecule que cette vie au désert: (Jue de bunnes qualites elle developpe, au physique et au mural, chrz Thumme, quand celui-ci est sullicamment orfanisé pour la pouwir mener?

Jo veux, a lappui de cetle réflexion, citor un trait de sang-froid et de prisence de-prit que jai vu accomplir a Bas-de-Cuir, un jour que mons chrssions dans la vallie de Bellata.

Xinu venions de faire lever deux sazelles à envirun doux remt- molres de nous. Je vouhis les lirer, mais Si-lisia me dit: a Elles sont deja bien filoignioss il e-1 profirable de les rahattre: preul-etre ainsi pourras-tu

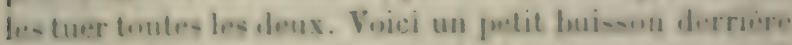

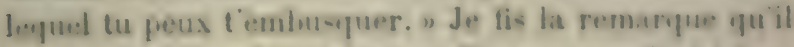

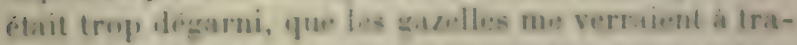
vers. "Cest srai, me dit-il: je vais tarraugur c..la."

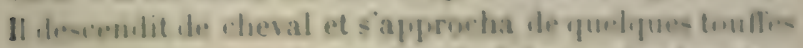

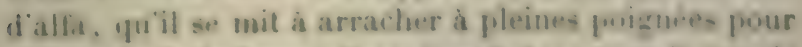
en garnir le bui-son. Il se livrait a ce travail depais

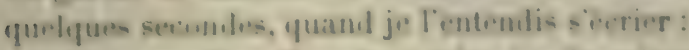

- Ih: maulite. Dien te pumen: o Je miapprochai frumr voir à qui il en avait. et je ris aree eftrai une wi.

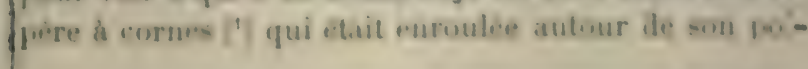

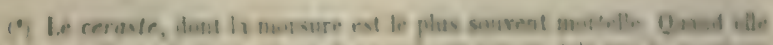

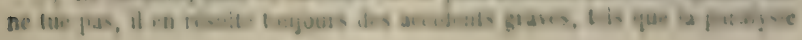


gnet droit; il la tenait à pleine main avec la poignée d'alfa dans laquelle elle se trouvait blottie, et la frottait aver vigueur sur le sol rugueux, lui usant ainsi la tête pour l'empêcher de mordre. Ce fut l'affaire d'un moment, après quoi il ouvrit la main et jeta à mes pieds le corps décapité de l'affreux reptile.

Sans cette subite résolution de frotter à terre avec force la tête de la vipère au moment où il s'était apercu qu'il la tenait à la main, Si-Aïssa aurait infailliblement été mordu; et Dieu sait ce qui serait advenu d'une pareille morsure, dans cette partie de l'avant-bras où abondent les muscles et les veines ! J'en avais la sueur au front. "N'as-tu rien? lui dis-je. Non, me répondit-il; elle n'a pas eu le temps de se reconnaître. Louanges à Dieu, qui m’a préservé! Allons! mets-toi à l'affutt; voilà ton buisson arrangé. Je vais tamener les gazelles."

J'admirai cet homme, dont l'émotion était à peine sensible après ce danger, et je n'osai lui dire que cet affût ne me plaisait guère. Je m'y plaçai néanmoins; mais, pendant que Bas-de-Cuir rabattait les gazelles, j'étais inquiet. Je me retournais souvent pour voir si la compagne du céraste, que je supposais près de moi, ne venait pas de mon côté.

J'ai toujours eu une grande répulsion pour les

complète ou partielle. Une cautérisation énergique et instantanée peut seule éviler ces accidents.

Les Arabes du Sud en sont tellement convaincus, que quelques - uns ont assez d'énergie pour faire l'ablation de la partie qui entoure l'endroit où ont pénétré les crocs du reptile.

J'ai ru, à Laghouat, un cavalier des Larbàs, qui, ayant été mordu p̧ar une vipère, s'était enlevé, avec son couteau à raser, la parlie charnue du mollet druit. Il boitait légèrement, mais il aimait encore mieux cela et vivre.

Quand l'ablation n'est pas suffisante, on y ajoute la cautérisat:on par de la poudre que l'on verse sur la plaie et à laqquelle on met le feu. 
reptites. J'avoue done que jetais loin d'etre calme; fillrilue a mon agitation l'insurers de cette embuscalle : les gazelles ne voulurent pas mapprocher: elles - infuirent, malgré les effurts de Si-Assa. "Gest un manvais endroit, me dit-il en me rejoignant, allons aillenurs. " J'ot is bien de son avis, et ne me fis point prier.

P'aure Bas-de-Cuir! comme jai plaisir a m non sm vinir: II a cté pour beaucoup dans mes surcies de rlasse du Sersou. Avec lui, il y avait toujours de lioriginal ef de limprévu. Notre pasision commune etahlissitit enlere nous la meilleure entente, je puis même dire une affection véritable. II completait Canaridville, et quand il fut entré dans notre intimité, il ny avait print de bonnes parties sans lui.

Line des plus grandes chasses que nous fimms damla grégion des plateaux eut lieu lautomme suivant puos la cébeluntion de la Saint-Hubert.

Partis de Canardville de 22 wetobre, en compraguie de Bas-de-Cuir, de nos compagnons de thas-e a coure habituels, les tils et neveux du bach-agha Ameur-lwen. Perhat, le calil des Doni-flatseni, celui des BeniManda, mon fidele El-Mebrouk et une vingtaine dautres cavaliers, nous fimes juspuia Taguine, en passant par les bous endroits comus, entre autres a loumb-0ury. ois nous trouvious toujours autant de péche que de chasse.

Voici les chilfres du gihier tué dans cette menusable tournée:

Gazelles................

Sianghers (rencositrés daus Kel-Mrtalis, pros de (ivuljila)

A reperter.

(i) $j \cdots$ 
Report. . . 61 pièces.

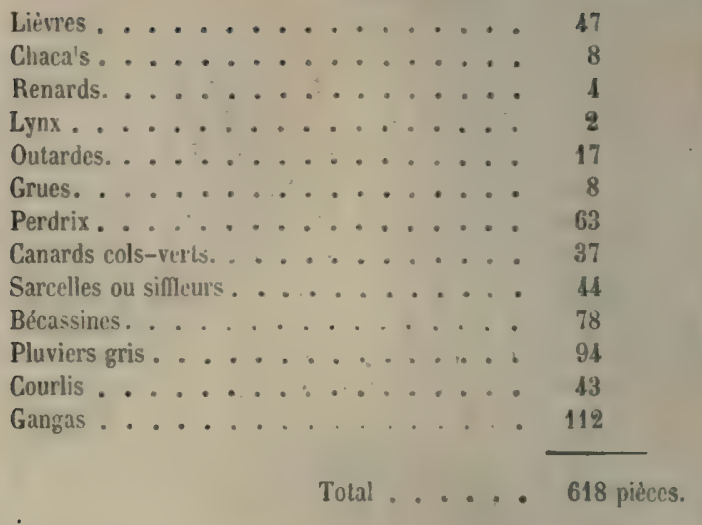

Nous étions soixante personnes environ. Pendant dix jours, nous avons vécu de gibier, et en aron: rapporté plus qu'il n'en fallait pour notre grand repas.

Dès l'année 1847, nous célébrions religieusement la Saint-Hubert à Téniet-el-Hâd.

Quinze jours avant le 3 novembre, on faisait circuler un tableau-liste, sur lequel signaient tous ceux qui voulaient y prendre part. Ce tableau, qui était composé par le plus habile artiste de la confrérie, représentait les principaux épisodes de chasse de l'année, arec ornementation d'accessoires et d'attributs à la fantaisie de l'auteur.

J'ai conservé la plupart de ces tableaux, qui font collection depuis 1850 jusqu'en 1857. Les signatures et les faits qui y sont représentés me remémorent mes compagnons de ce temps-là, des chefs aimés, dont beaucoup ne sont plus de ce monde : 
Parmi ceux-ci, les marichaus Buswaud, I'-liesicr, Bosquet ;

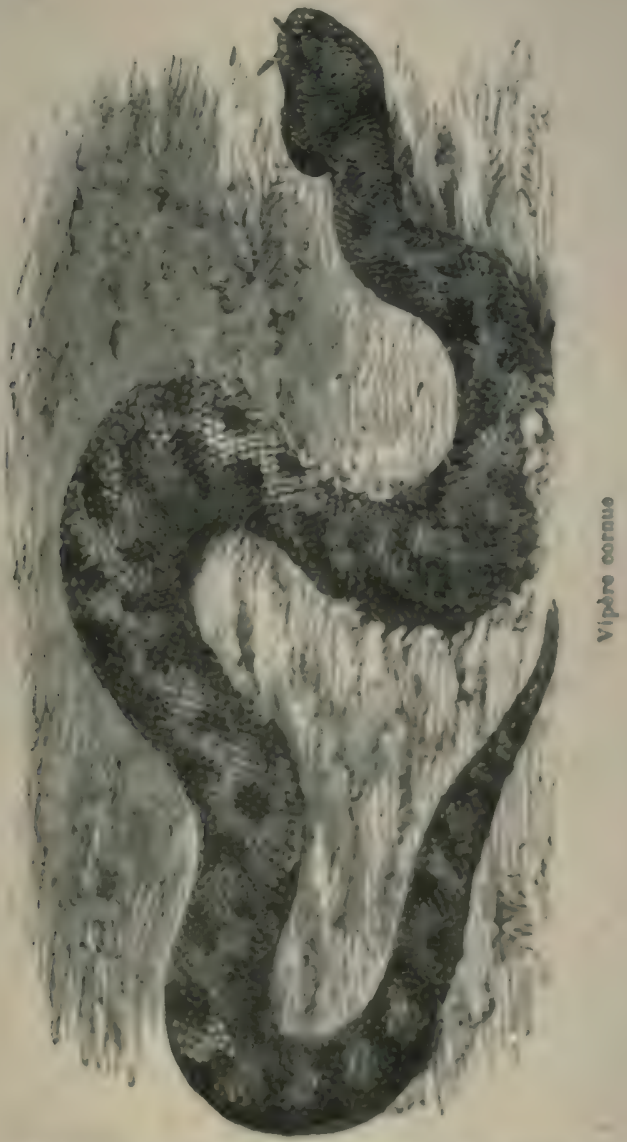

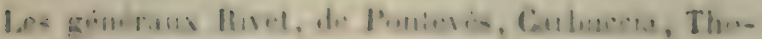
1:is, Clet, C.1sonigualles, Yusuf, 
Mon vieil ami Kennedy, mort en 1864 colonel et commandeur de la Légion d'honneur.

Parmi les généraux actuels qui ont chassé à Canardville il ne reste guère que le général de Fénelon, qui pourrait se rappeler certaine traque au lièvre en compaguie de notre bien regrelté général Rivet, le brouet saharicn du vieux Rahal, et surtout cette fameuse course de trente lieues en huit heures que nous fïmes, de Canardville aux Beni-Zoug-Zoug, à la poursuite du cherif ${ }^{1}$ Bou-Touala, à travers un pays boisé et montagneux;

Le général Bataille, qui ne peut aroir oublié nos chasses à la gazelle dans le Sersou, et nos bonnes stations à Lapinbourg.

Je velix, du reste, pour n'omettre personne, mettre à la fin de ce chapitre les noms inscrits sur les tableaux de nos Saint-Hubert. Maître Paul, après moi, aura plaisir à les lire.

Tous ceux qui prenaient part à ces fètes n'étaient

(') Cherif, noble, nom que les Arabes dunnent aux descendants de Fatına, la fille du leur prophète Mohamed. Par extension, ce nom de chérif a été souvent donné aux agitateurs qui ont voulu se faire passer pour le moul-elsaa, le maître de l'heure, le prédestiné envoyé par Dieu pour anćantir et expulser les clurétiens de l'Algérie, selon les prophéties accréditées chez les Arabes.

Un de ces plus célèbres agitateurs a été Bou-Maza, l'homme à la chèvre. Mais combien d'aulres, sous le nom indiqué dans les prophéties de Mohamed-ben-Abdalla, ont troublé l'Algérie en causant des insurrections partielles qu'il fallait vite étouffer sous peine de les voir grandir comme ces incendies qui ont à leur portée des matières inflanmables à dévorer !

Le ròle de ces chérifs est tombé en discrédit, aucun n’ayant réussi jusqu'à ce jour à amoindrir notre domination en Algérie.

Mais il ne faut pas se hâter de conclure qu'il ne sera plus joué à l'avenir.

Il tentera encore bien des têtes exaltées, et trouvera, dans certaines circonstances, bien des adliérents. Ne pas perdre de vue cette éventualité sera sage de notre part. 
rims chasseurs, mais pour la circonstance chacun en fremait le titre et les allures.

II etait de tradition de ne ennommer, dans res granı!'s repas, que du gibier tué par tous les adhérents. Huit jours avant, on se partageait les tàches, - qui allait a la grosse bète, qui au marais, qui an gihier orilinaire. Enfin les simples mazettes elles-mémes faisaimt arte de bonne volonté en récoltant dans les champ des environs quelyues douzaines dalouettes, de bees-fins, des champigmons, du cresson, de la -hicoree, et en pechant dans la rivitre des larbeaux et des crabes.

dinsi, depuis le polage jusiguau rit, le repras de Siant-Hubert était le produit de la cooperation de tous. II n'en avait que plus d'attraits dans une lusalite nu it nuns aurait etis difficile de trouver autrement les mets d’un festin.

Voiri un de nos menus: il donnera une idlee de la manive dont nous nous traitions en festoyant nutre grand patron.

DINER IE LA SAINT-HUBERT DE 185 A.

porages.

Consommé aut gangas.

Bispue de crales de rivitre. 


\section{ENTR RंES.}

Civets de lièvre du Sersou.

Matelolte de barbeaux à la marinière.

Timbales de perdrix aux choux.

Salmis de bécassines.

Rảles de marouettes à la chasseur.

Còtelettes de lapereaux à la saharienne.

Grues en dauhe.

Sarcelles braisées aux olives.

Filets de sanglier aux terfès (truffes blanches des liauts plateaux).

IIOTS.

Outardes farcies de becs-fins.

Cols-verts, bécasses.

Cuissots de gazelle.

BOUTS DE TABLE.

Jambon de lion, lure de sanglier.

Pàté froid de pluviers.

\section{LÉGUMES.}

Champignons, chicoréc, oseille.

\section{viNS}

De Milianah, de Bordeaux, de Champagne.

Ces banquets annuels, où régnaient l'entrain et la gaieté, étaient de joyeuses fêtes qui occupaient, quinze jours à l'arance, tout le personnel de la garnison. Ils mettaient un peu de variété dans l'existence forcément monotone des postes avancés, et, comme conséquence, ils cimentaient la bonne camaraderie en établissant entre tous ces bonnes relations, qu'il est si désirable de voir régner entre gens qui concourent au même but. 
Dans cette rivière, où l'on pouvait prendre d'exccllents bains dans la saison chaude, se trouvaient en profusion des barbeaux, des anguilles et des crabes. Nous pouvions alterner nos chasses par de jolies pêches, et nous ne nous en faisions pas faute.

Il reste toujours un peu du sauvage dans l'homme civilise. C'est ce qui explique pourquoi nous avions tant de satisfaction à vivre de notre propre industrie en prenant aux bois, aux champs et aux rivières notre subsistance quotidienne.

Quels riches tableaux de genre on aurait pu faire de nos bivouacs, si animés par la présence d'hommes affublés de costumes différents, avec nos chevaux, nos chiens, des guirlandes de gibier pendues aux arbres arec nos accoutrements de chasse et nos armes!

Le soir surtout, quand de grands feux étaient allımés, que la flamme éclairait le feuillage des arbres, les groupes variés de gens et d'animaux de toutes les attitudes, et que le repoussoir des ombres dégradées jusqu'au noir sombre de la nuit y mèlait d'étranges el magiques effets, il y avait de quoi inspirer les natures les plus rebelles. Combien nous regrettions alors notre impuissance à fixer par le pinceau ces tableaux qui nous charmaient!

: Le moment encore aurait été bien choisi, quand le rugissement d'un lion ${ }^{1}$ se faisait entendre près de nous.

les chefs-lieux des provinces que l'émir entendait gouverner, malgré notr conquête des villes de Mlédéah, Milianah, Mlascara et Tlemeen, auxquelle: ces forts correspondaient.

(') Nous eùmes trois fois l'occasion de faire ces remarques dans différent: séjours à Lapinbourg. Une fois surtout, le lion vint se désaltérer, vers neu heures du soir, dans la rivière, à quarante pas de notre bivouac, ainsi que nous pùmes le consiater le lendemain par ses traces. 11 rugit à trois reprises. sans doute à l'aspect de notre bivouac très éclairé, et si violemment que nous crûmes à une agrussion. Clıacuu prit son fusil et se mit en déiense; 


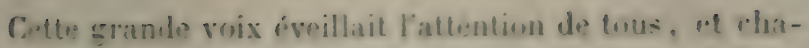
run, a ce unment, traluisiat son impression selun sa I1.1ture.

Les hommes faisaient bunne contenance. lunt en se romarilant furtivement pour imiter lattitule des muins impresimmes, car l'amour-propres ne juerol jamais ses druits au eranul juur ou a la lumire. Les chevaux tendairnt lenedure. regardaient dans la direction du danger, acpiraient lair fortement, en soullant a pleins nascaux, wt se cabraient pour se dobarrasser do lesurs entraves. Less chiens entin venaient se refugier dans nus jimbes en hurlant. ou aver des abovienents de menace et de crainte.

1)ui. tout cela aurait fait dadmirables tableaux: mais mous rtions inluabiles a les reprowhire. Malere la tentation, nul niaurait pu ripriter avee suerès lo mot du Corrige : a Et moi aussi je suis peintre : o

los touri-los franc ais et etrangers qui venaipht exphorer le prays etaient egalement emerveillos du site do. fapunibourg, du eachet de grambe arienalite qu il reviblut, yuand nous l'anjmions de nos bivouacs.

D. ceux qui vimrent ehamer avee unus, jai purtioudierenuent garde le somenir de desux Anslais qu ma-

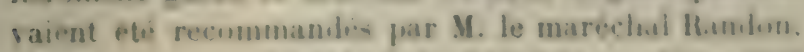
alurs goumerneur general de l'llgeriee. - Lun etall

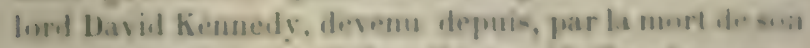

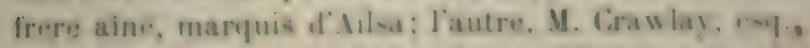

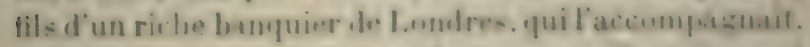

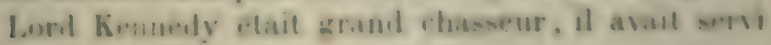

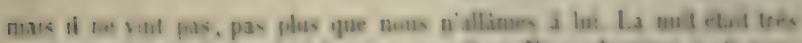

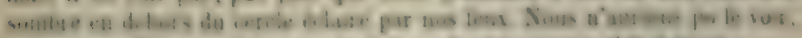

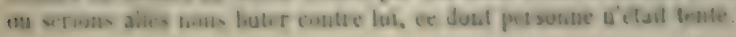


plusieurs années dans l'Inde et y arait tué bcaucoup de tigres. Sa grande ambition était de luer un lion. Je m’associai à ce désir, que je trouvai légitime; je tis sout ce que je pus pour lui procurer cette satisfaction, mais sans y pouvoir réussir. Affûts souvent répétés dans les meilleures conditions, poursuite des lions en suivant leurs traces fraiches dans la neige, rien n'y fit. Une fois seulement, lord Kennedy entendit le lion gronder à quelques pas de son affût, mais il ne put le roir, ni, par conséquent, le tirer. Ce n’était pas assez pour tant de peines.

Après chaque déception, et comme pour s'en consoler, lord Kennedy évoquait les souvenirs de ses triomphes de l'Inde, il me proposait d'y aller avec lui, tuer des tigres. J'y étais déterminé. Peut-être aurions-nou. réalisé ce projet, si la guerre de Crimée n'était venue modifier nos résolutions:

Lord Kennedy et M. Crawlay restèrent près de deux mois à chasser avec nous. Il va sans dire que je les conduisis dans nos meilleures réserves, y compris Canardville et le Sersou, où ils prirent grand plaisir ì la chasse à la gazelle.

Iis étaient pourvus, comme tous les Anglais le sont :énéralement, d'excellentes armes. Lord Kennedy surtout possédait une carabine de Lancastre, avec laquelle je l'arais vu tuer une gazelle à sept cents mètres, dans un petit troupeau d'une douzaine de têtes.

Nécessairement, j'étais devenu amoureux d'une arme pareille, surtout après l'avoir expérimentée. Je n’eus de satisfaction que lorsque lord Kennedy me promit de m'en faire fabriquer une semblable, tout en me prévenant "que Lancastre était un grand paresseux » et mettrait plusieurs mois à celte confection. 


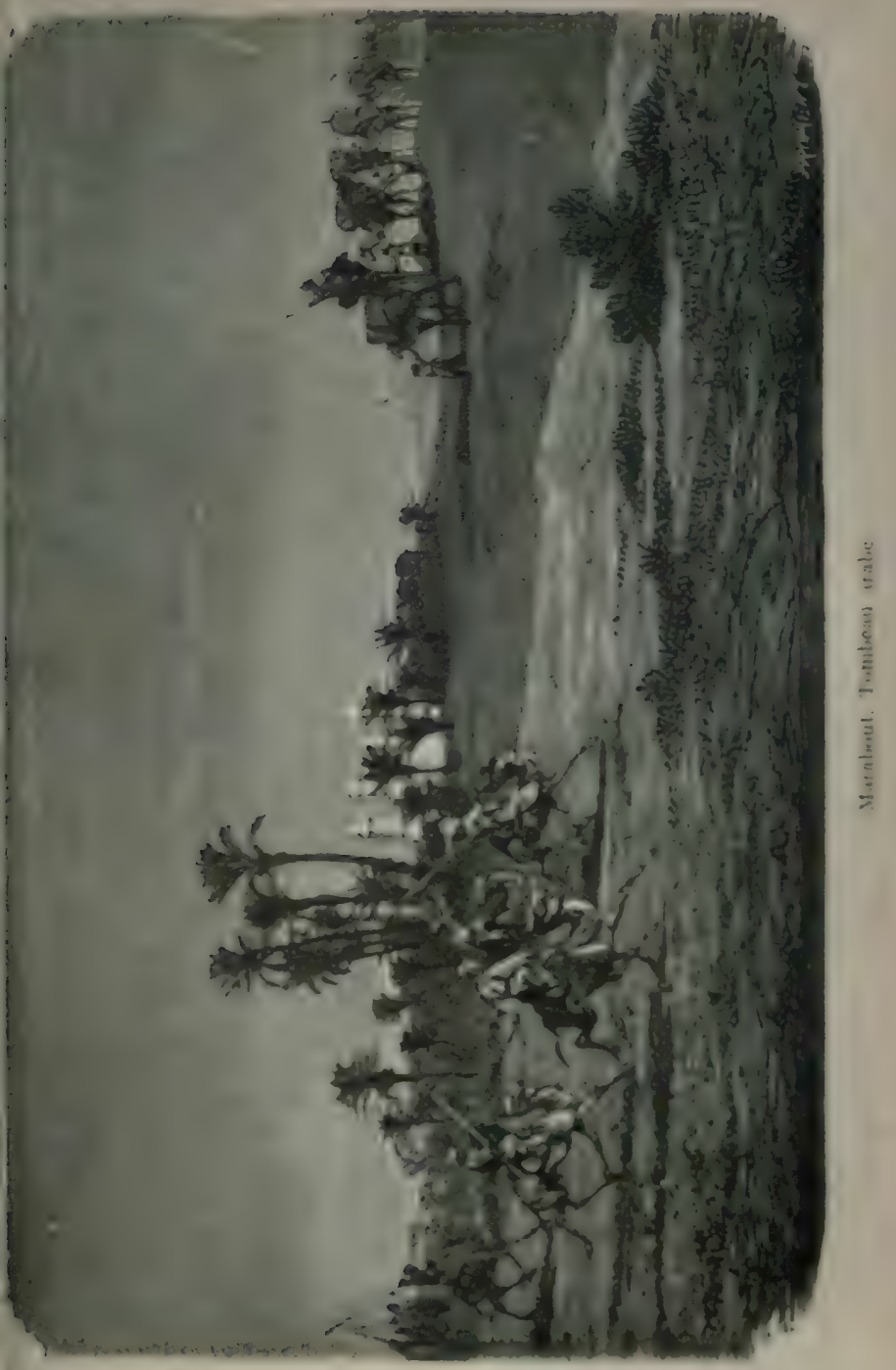



Gornme je me rieriai sur la longuenr du délai, il mexplisqua que cet aronurior conserencinux ne livaif ses armes quiaprés une sirie d'iprenves et avoir régle lui-meme, fendiant plus de trente jours, au tir, la porsition de l'encuche, de la hausse et du guidon. - Ce ufui expliquait la juste-se de sa carabine jusigu a la distance de mille yards environ gok metres.

Je reçus effecturement six mois plus tard cetle excollente carabine, avee layuelle je ne manyuai pas de miesercer. Je prenais plaisir a faire awe elle les paris los plus arentureux. Jen gagnai un, entre autress a M.M. de Biemeourt ef ite Ludre. qui vinrent a relle ipuque visiter laghouat. Cela me fit grand plai-ir. car it retairnt assez bons tireurs pur qu il y ent sati-fisetion a les vaincre. lienju, qui elait un panier de bordeaux. ful, du reste, conscrienciensement hu a leur saute.

D. I.apinbourg, il nums arrivait assez fréfur.mment ana-i d. faire des pelites pointes vers les endroits yui

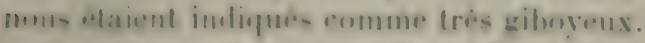

Xuns denciondinus alors loued Deor-Denr, fertile en

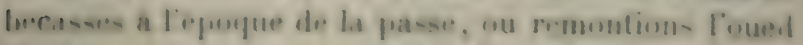
Monullat, le pass far escellene des perdrix.

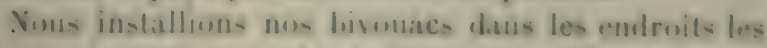

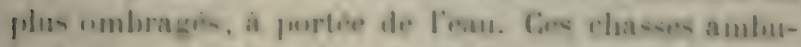
lanter, par le firit meme du deplacement, etaient ume

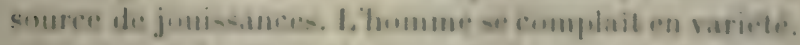
celit est certain. Nous aimions a fouler tous lwo jours un sol nouseau, d asperts diflerents. prenple d aul res genr..

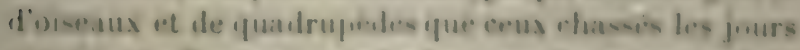

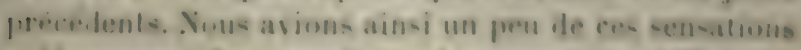
qu cpromsent les stamels explurateurs quand its conrhent une terre vierge et decouverent of gue dautres niout fats trouve arant eux. 
-Ouni! pas une déception, un insuccès, pour varicr cette constante sírie de réussites?

- Si, monsieur Paul, en voici une que je veux vous dire ici, sans préjudice de celles que je rous conterai plus tarı.

Un jour de l'automne 1833, le capitaine Bouchot, du

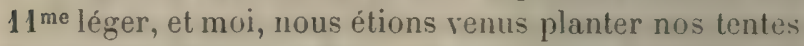
à Lapinbourg pour y faire une bourriche de gibier qui devait servir à notre repas de Saint-Hubert.

Nous avions déjà une jolie collection de lièvres, perdrix et bécasses, lorsque, dans la matinée du troisième jour, en parcourant la partie supérieure de la vallée, nous fimes la rencontre de El-Mokhtar-Bel-Arbi ( ${ }^{1}$ ), accompagné de deux de ses cavaliers el de quelques piétons des Matmatas. Il renait me roir, sachant que jétais à chasser à Bou-Radja.

Il me raconta qu il avait quitté sa tribu depuis trois jours pour suivre les traces de deux lions qui, en traversant le territoire des Beni-Mahrez, lui avaient tué une jument et lionné un de ses serviteurs.

J'avais déjà entendu raconter de ces histoires de gens lionnés, mais je n'y arais accordé qu'une mince criance.

Ainsi, on m’avait affirmé, chez les Blal et les Matmatas, que des individus, dont le moral n'était sans doute pas d'une trempe énergique, avaient été rencontrés isolément le soir $\left({ }^{2}\right)$ ou la nuit par des lions, et soumis de la part de ceux-ci à une sorte de fascination, dont l'effet était de se faire suivre par le patient, qui devenait alors estesebâ, lionné, comme nous disons médusé $\left({ }^{3}\right)$.

(1) Voir la chasse au lion, page $\mathbf{1 5 .}$

(2) Les lions ne sortent pas de leurs repaires dans le jour, et il est rare d'en rencontrer avant le coucher du soleil.

(3) Dans tous les récits de ce genre qui m'ont été faits, je n'ai pas trouvé mort d'homıne dans le souvenir de la génération présente.

Les Aralies prétendent néanmoins que le lion, après avoir entrainé l'homme 


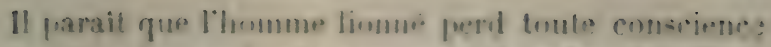
de -a -ituation vraie et ses soumet a la volonted du linen. qui le fromene lit wi il veut. Des serns ayaut de rencuntries dans cet elat disaient a reux qui voulaient le-s ramener: "Laissez-moi conduire mon lueuf. Vous voyez. hien quil s'enfuit et que jes vais le perdres. ou dautres flrugos aus-i incohérents. mais qui indiquaient texjours La presecrupation de suive le lion fasinateur.

le remeile, en pareil cas est dappliguer de vigouraux soufllets sur la face des lionnés, yui se réveillent alurs plus on moins de celle violente oborsion.

Ciette croyance au limnage est tellement ancriditée cherz les Arabes qui habitent les parties besisties du Tell. quilis emplonent à l'orcasion, pour s'y soustraire. le moyen suivent: sils sont a cheval et armés, ils tirent Jeurs sabres ou bramlisont leurs fu-il-. font caracolin leurs rhesaus, frappert de leperon contre leurs larews

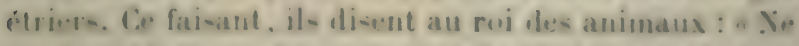
me connats-tu gas? jo suis un tel, fils d'un tel, la terremer de mes ennemis: Passe au large, tu nias rien a gagner avec moi.n

te lion, vogant a qui il a afficire, après deox ou trois

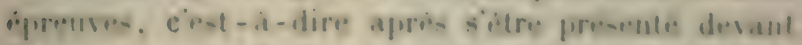
f'homme, sur sa droile ef sur sa gauche, sans parsenir 'a rintimialer, finit par le laisoer.

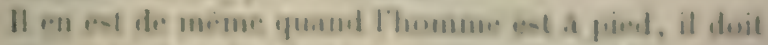

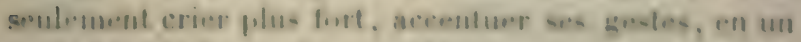

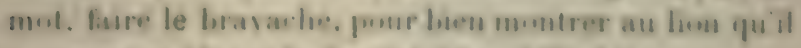

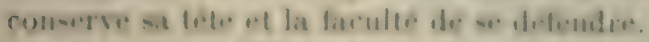

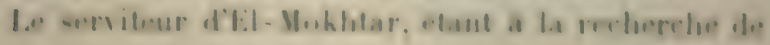

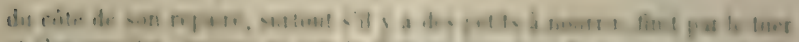

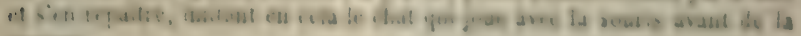
manger 
la jument de son maitre, avait rencontré deux lions, à la tombée de la nuit, dans un lieu boisé et éloigné de toute habitation. - Il avait sans doute éprouvé une grande peur à la vue de ce couple royal, et par suite une absence qui l'avait laissé inconscient de ce qu'il faisait.

Heureusement pour lui, il fut trouvé dans cet état par deux Arabes des Beni-Mahrez, qui étaient aussi à la recherche de bœufs égarés; ils n'hésitèrent pas à lui appliquer le remède des soufflets, et si vigoureusement, paraît-il, que notre homme, étant revenu à lui, avait pu dire sa fâcheuse rencontre et montrer l'endroit où il avait vu les lions, etc.

Il n'y eut aucun doute, du reste, quand on vint dire le lendemain à El-Mokhtar que sa jument avait cité retrouvée un peu plus loin, à moitié mangée, sur la limite des Matmatas.

Jamais personne, de mémoire d'homme, n'avait perdu autant de bestiaux que lui par le fait des lions et des panthères. Il semblait que ces animaux eussent une rancune toute particulière et bien justifiée contre ce grand destructeur de leur race.

Il m’avait fait un jour le dénombrement de ce qui lui avait été mangé; le chiffre s'élevait à une vingtaine de bœufs ou vaches, à une soixantaine de moutons, et cinq poulains ou juments.

La dernière tuée était une bête de valeur : aussi ElMokhtar cherchait à retrouver les auteurs de sa mort pour leur faire expier ce nouveau méfait.

On lui avait donné des renseignements qui lui faisaient supposer que les lions devaient être assez près dans les environs : l'avant-dernière nuit, ils avaient e:llevé une brebis dans un douar peu distant de nous. 
Je proposai it El-Mokhtar de venir dejeuner, apris quui nous avigerions à continuer les recherches, aux'fueiles je me proposai de prendre part.

Xous avions déjà fait plus de la moritié du chemin funr regagner notre bivouac, lorsque nous vimes deux Arabes apparaitre en eourant au sommet d'une colline yui se trouvait à notre droile. Dàs quils nous aprercurent, ils nous tirent des simnes avec leurs burnous, en nous criant de les attendre.

IIs paraissaient tres emus et avoir quolyue chose d"imprortant is nous communiquer. Anssitot qu'il-furent frris de mous, ils nous dirent:

- Jeux lims viennent de tuer motre anesse et sun pretit. Ils sont en train de less manger sur le versant "ppose. Si vous roulez les frapper, vous nivez pas de (1.0ups à perdre, nous cous conduirms vers enx. .

Cefte nerasion nous parut si favorable de joimlre mos frux malliatrurs, ear ce ne pouvait etre qu enx, que muns tuns mimeg en marche sur-le-champ. Le capilaime bourlunt avait voulu otre des notres themin faisant, unus chargeànes nos fiusils at balles.

Arrives sur la crete, fes Arabes neus montrirent, de lantro pite din ravin, a environ deux rents mitres. un gros buiseon, dans lespel ils nous aflirmerent avoir viu les lions ocenpés, quelyues minutes av ant, à manger l'inesse of ron petit.

Di-Mokhlar mit pied a terre, laiesa la sa monfure ot sint avee mous, ainsi que mus deus guiles, yui mon menirent sur le huisoon. Xuns fimes te moins de liruil pussible en auss dis-imulant derriere les touffe- de lintisques of de genevriers dont la pente de la colline élait boiscée.

Nius eperions, de celle mantere, approcher lons lione 
ocrupés à se repaitre, les voir el les tirer le mieux possible. Mais, arrivés à dix pas de l'endroit où ils devaient ètre, nous eûmes beau écouter et regarder avec attention, il n'y avait rien... que les deux înes lués, déjả fortement entamés à la partie du cou et des épaules.

Sans doute, les lions nous avaient vus apparaitre au sommet de la colline et avaient momentanément abandonné leur repas.

Nous étions à faire ces réflexions, lorsque nous entendimes un rugissement a deux cents mètres de nous. dans le fond du ravin.

"Ciest cela, me dit El-Mokhtar, nous les avons dérangés; mais ils ont encore faim, ils reviendront quand ils nous croiront partis. "

Nous fümes alors d'accord d'ćlablir immédiatement des alliits pour nous placer dedans et attendre leur refour. Nous nous mimes it l'œurre aussitût; nos deux Arabes étaient pourvus de leurs garloums ( ${ }^{1}$ ), qui nous servirent à faire deux trous, entumés de branchages: dans l'un devait se mettre le capitaine Bouchot, dans lautre, El-Mokhtar et moi.

Puur tirer parti du terrain, selon ma façon de faire habituelle, de mettre laffît en contre-bas de la bète à tirer, javais, pour aller plus vite, établi le mien dans le creux d'une ravine. Inspiration médiocre, comme on va voir.

Notre travail achevé, les deux Arabes étaient partis avec mission d'emmener au bivouac le cheval de Mokhtar et cclui du cavalier qui devenait le compagnon du capitaine Bouchot; puis nous nous étions placés dans

(') Instrument qui est pioche d'un còté et hache de l'autre. Presque tous les montagnards en sont pourvis et le portent à la ceintur'. 


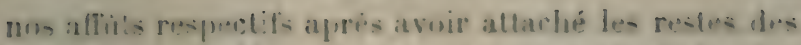
dend anes en dehors du fonrré, sur lendroil le plas propice in notre tir converarent.

11 netat quine heure de lapris-midi, nous espirrimas que les limss nattemelraient pas la nuit prour trevenir. Il sureltaiput sans doute nus mourements: lor-

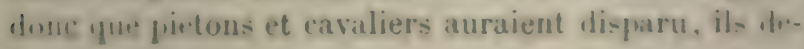
vaient arriver.

It mons semblait, tant uous étions convaincus d'ume. réu-ite. que ce netait plus quire question de patti.r.w.

Mais deux heures, trois heures s ienulirent, el rien ne viut. Pous commenaciuns a trouver le temp-long, a noms impatienter, lorspue nous entemdimes des rugi-

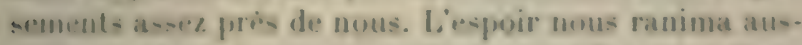

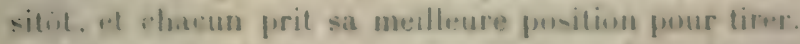
Ce fut en vain : les lions ne parurent pas. Nius les entemdions erpendant qui se ripuntaient a une trontaim.

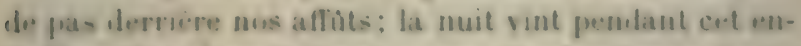
trotien. dans leppuel dis se communiquêrent sans doutr linure impirmalums.

Bue funvarent-ils se dire? Eidemmeat ils navairnt pa- i.t cerlitudes de motre prisence: mas queldue chune d'insulite les précempait. Elaient-ce nus aflists. que

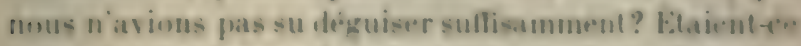

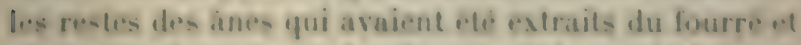
phares quelques pas plus haut, qui lecur hisuent ir.

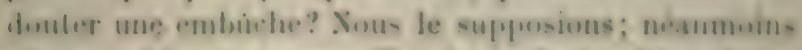

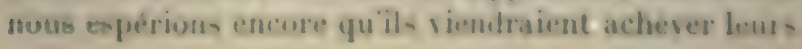
reliefs.

C.e qui nous le faisait rroire, cimbl que, depuis quu. la nuil etait veume ils sitaum rapproches, sans que endeliois nous pursious les ruir. 
Le temps s'était couvert vers quatre heures de l'aprèsmidi; au coucher du soleil, la pluie se mit à tomber sous forme de brouillard très épais. En dehors donc de l'endroit où gisaient les ânes, lequel se détachait assez nettement dans le ciel, le reste des alentours se trouvait dans une obscurité profonde.

Et nous étions là, toujours, le fusil à l'épaule, une main sur les batteries, pour empêcher la pluie de mouiller les capsules. Les lions rôdaient et grondaient, mais ne se présentaient pas au but. La pluie tombait en augmentant d'intensité, de facon que, vers huit ou neuf heures du soir, nous sentimes, El-Mokhtar et moi, 'ju'un petit ruisseau entrait dans notre affût.

Je dois le dire, l'idée de nous en aller nous vint à ce moment, mais ce n'était pas possible: ces maudits lions nous maintenaient en espoir. De temps à autre, ils rugis:aient ou grondaient comme de gros chats auxquels on veut enlever la viande qu'ils ont volée. Nous les entendions frôler les buissons dans un rayon d'une vingtaine de pas autour de nous. Mais l'eau aussi montait dans notre trou, profond de deux pieds, sans moyen de l'éviter, à moins de sortir de l'affût, ce que nous ne roulions pas faire. Vers minuit, elle finit par le remplir tout à fait, et nous nous trouvàmes dans une véritable baignoire des plus réfrigérantes.

A cette heure, nous étions trempés jusqu'aux os; la partie de nos individus qui se trouvait en dehors du trou n'avait rien à envier à celles qui étaient soumises à la baignade. .

Nous restâmes ainsi jusqu'à cinq heures du matin, connant un rare exemple dentêtement dans une situation qui n'avait rien de récréatif, on peut le croire; d'autant plus que nos estomacs étaient tiraillés par la 
faim. puner naveir tren mangés depuis plus de vinglquatre laeures.

Mais, je le réprate un toute conviction, il n'y anait fias d'aulre parti a prendre que de rester dans nus alfüls jusina un denmement avounble : ou la mort di-s lions, ou leur retraite. Cela était derenu une iqueslimn il amuur-propre entre ces bêtes el nous.

"Xous voudrions arhever l'änese et sion petit ". devaient-elles se dire.

Nous répontions tacitement, nous, à la maniere de Leonidas : "Ciest possible, venez les prentre."

Notre vaine percévérance finit par l'empurter, les linns ne vinrent puint. Vers trois heures du inatin, its - Celuignèrent dans la direction du Djebel-Trhatoun, or que nous comprimes par les rugessements, yui sideiguirent graduellement. Mais, pour bien maryuer nober rageuse tinacite, et aussi par un fol espoir quils pourratent se raviser of revenir, nous attendimes deux heures encore.

linfin, Paube prarais-ant, uens sortimes merfondus de noss baigmoires, ell deblatérant contre ces crelin- de lions qui araient ile assez, stuphes frour ne pas venir moratre à cess pauvres âne- quils avaient -i mallownotement expetilies de vie a trépas.

" Lies tils d'infideles, disait El-Mokhtar, nous ont devinés, ils sien cunt avee leur peau et leur morgue. lenr jour nelail pas arrive. o

Puila ce yue notre mauraise humeur nous laisail lire dans ce monnent. Mas plus tari, apores avoir monint notre bivouar, nous the sibhes et re-taurés. suus ềmes une meilleure opunion de la sagarcité de mus deux lions, ou du moins nutre maniere de nous exprimer à leur endroit fut plus révérenciense et plus juste. 
El-Moktar, qui avait contre eux une rancune ligitime, se mit dans la journie à leur poursuite, jusque dans le pays des Oulad-Antar; mais sans le moindre risultat, ainsi que je l'ai su après.

Le capitaine Bouchot et moi, nous rentrâmes le lendemain à Téniet-el-Hâd avec une cargaison de gibier, et, chose plus appréciable après notre fraiche nuit, sans le moindre rhume de cerveau.

Eh bien! ce souvenir, qui n’est pas précisément celui d'un succès, ni une réminiscence des délices de Capoue. m'est, à distance d'une quinzaine d'années déjà, particulièrement agréable.

Il en est ainsi dans la vie; nous nous remémorons plus volontiers peut-être les mauvais jours, les misères subies et bien supportées, que les plaisirs et les joies qui sont venus facilement à nous.

En déduire toutes les raisons mènerait loin. Mais, pour abréger, ne peut-on voir dans ce fait un stimulant des plus propres à nous faire mieux accueillir les épreuves successives de notre avenir?

Conclusion et morale: Il faut en ce monde receroir avec résolution les douches que la destinée nous enroie, afin d'acquérir une meilleure trempe.

Voilà, mon cher Paul, un axiome que je vous recommande tout particulièrement, en arrêtant ici cette première partie de mes souvenirs.

J'aurais encore beaucoup à narrer sur Canardville et Lapinbourg, sur les autres chasses, de mon long séjour à Téniet-el-Hâd. - Je pourrais y ajouter d'autres récits et d'autres souvenirs d'une période de cinq 


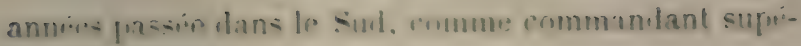
rieur de Laghouat.

Peut-atre mien aviarai-je un de ces jutrs, quand votre frere Victur vientra a eun tour me difmander des

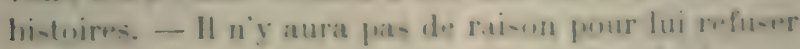

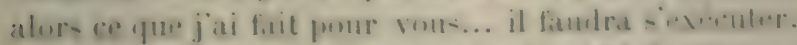
- Finatlemtant. et -elun un mot qui a deja du elurm. [und vos orrilles, - je me dunne campus!

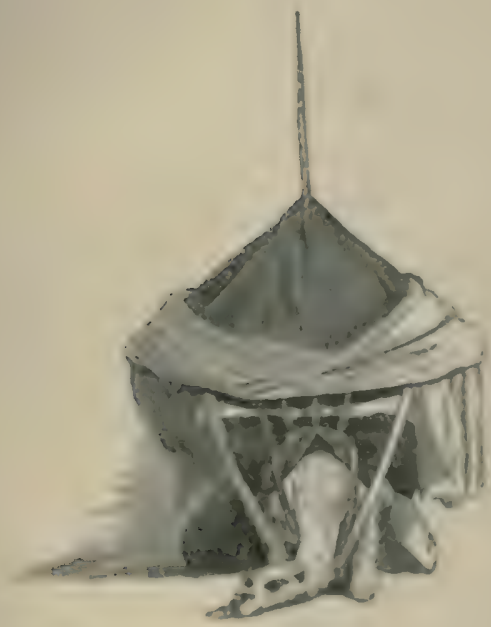




\title{
LISTES DES SOUSCRIPTEURS
}

\author{
SAINT-HLBERT DE 1850.
}

(TÉNIET-EL-HAD.)

Kensedr, commandant supérieur de Téniet-el-Hâd. - Mangevnitte, clief du bureau arabe de Téniet - el-Hàd. - DE MArhas, chef d'escadron de spahis. - Depotet, Klenc, Cocé, Gérard, capitaines au 2e bataillon d'Afrique. - Barrage, Régad, (apıtaines du génie. - Pavier, docteur. - Semziat, lieutenant adjoint au buregu arabe. - Casse, docteur. - Gocctis, Couté, lieutenants au $2^{e}$ bataillon d'Afrique.

\section{SAINT-HUBERT DE 1851.}

$$
\text { (TÉNIET-EL-HAD.) }
$$

Kexvedy, commandant supérieur de Téniet-el-Hâd. - Mlalgienitte, chef du bureau arabe de Téniet-el-Hàd. - De Maruan, chef d'escadron de spahis. - KLENc, capitaine au $2^{\circ}$ bataillon d'Afrique. - De Moynier, tonriste. - Pellemin, Lassts, docteurs. - Thomas, capitaine du génie. - Seriziat, lieutenant adjoint au bureau arabe. - Grimald, LÉo, officiers d'administration. 


\title{
SAINT-HCBEITT DE 1852.
}

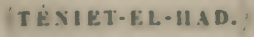

Kavrar, chef de bataillon an $33^{\circ}$ de ligne. - Marctratrr. rommandant suprerieur. - Boccusot, eapilane au 11 e leger. -

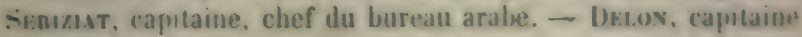
commandant la plare. - Tuosas, capulane du fruie. - Ginste. oflicier didunistration. - Cuttri.le, lieutenant au $\$$.

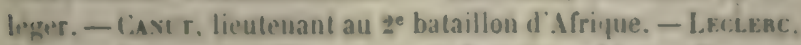
Lissis, docleurs. - Roziza, capitaine de spahis. - Bneros, lientenant. - Niconas. - Lalok, uflicier dadministration. Detatxk, lieutenant.

\section{SAINT-HUBEIT DE I853. \\ (TKXIET-RL-MAD.)}

Mum, f:rtrv, commandant superieur. - Butchot, capilain. au 11 - liggre. - Tuomas, colonel du 11 e legger, commandant la

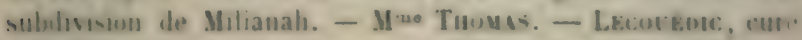

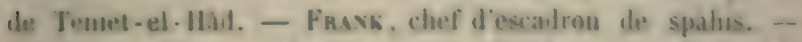

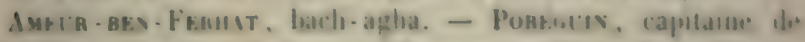

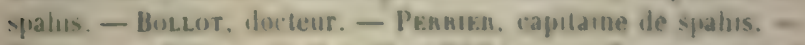

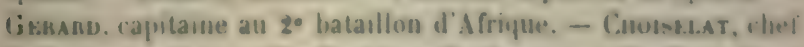

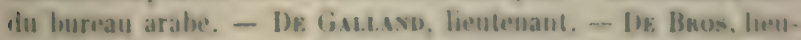

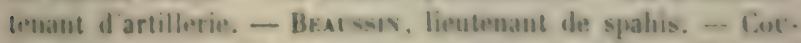

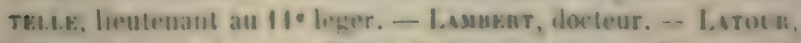

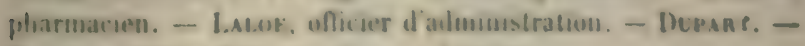
Prour, raputaute d'elat- major. - Arsanto.

\section{SAINT-HLEBERT UE 1856.}

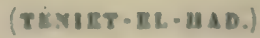

Batanle, colonel, commandane ha subdivision de Milianah. - 
Mangcenitre, commandant supérieur. - Elward Coorfar, oflicier anglais, grenadiers de la garde. - E. Hugart, major andans, 70 hussards. - Si-Sumex, agha de Milianah. - Si-EL-H.ubis, acrha des Braz. - Baron LAganoe, capilaine an fer spahis. Botrcenet, capilaine, chef du Jureau arahe de Milianalı. Horbigant, capitaine du genie. - Lemondan de Lavgornax, capitaine adjudant-major. - Beatssıx, lieulenant de spahis. Mavcue, docteur. - GaYtTe de Tévi, officier comptable dlalministration. - Lecocḱdı, curè de Téniel-el-Hàt. - AnELen, chef du lureau arabe de Téniel-cl-Ilàd. - PoвÉgtıx, capitaine

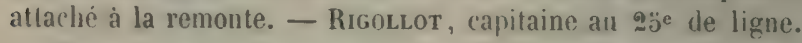
- Decos, commandant de place. - De Lassalle, capitaine de spahis. - Ozaxweau, lieutenant. - Richer, garde du génie. Si-Ahmed- Menzogga, Kholja. - J. Play. - E. Bertrand, capilaine. - Mocx. - Grsta, ofticier d'administration des hôpitaux. - A. Doter. - Bex-Omar, lieutenant de spahis.

\section{SAINT-HUBERT DE 1855.}

\section{(LAGHOUAT.)}

Margcentate, commandant supérieur. - Viscent, capitane du grinie. - Hollecx, Frère, capitaines au ze bataillon d'Afrịue. - Hensaxt, capitaine au fer tirailleurs indigenes, - DE PIsA, Deschamps, lieutenants de chasseurs. - Guérard, officier l'aclministration des hôpitaux. - Bracssıx, capitaine de spahis. Canms, capilaine de spahis, chef du bureau arabe. - Ch. Loysn, curé de Laghouat. — Duraxd, capitaiué au 2 e bataillon d'Afrique. - Robert, officier d'administration. - Mecxier, garde du génie. Bocderba, interprète militaire. - LE Bissonisais, lieutenant adjoint. - Deux signatures illisibles.

\section{SAINT-HUBERT DE 1856.}

(LAGHOUAT.)

Margueritte, commandant supérieur. - Vivcest, capitaine 
In grinie. - Carnes, capitaine de spahis. chuef du burean arabe. - Frisefe, capilaine au qe bataillon d'Afrique. - Mecsien, earde du geuir. - Besussis, capitaine de spahis. - Génnand, uflicier d'allministration des hojpitaux. - BocDeBBa, interprele milıtaire. - Segretaiv, capilaine du géife. - Faligu, lientenant au fer firailleurs. - Pel.1sstea, pharnacien de l'höpital unilitaire. Tissizr, docteur. - A. Faivre, Algar, Ginmd, Costaner. lieutenants. - Gruor, lieutenant adjoint au buraa arabe. A.-G. Boosazmr, docleur.

\section{SAINT-HUBERT DE 1857.}

(LAGHOUAT.)

Marcienttrs, commandant superieur. - De Rrascer. le barun dillooglagnd, De Bonely, touristes. - B. Fuchsien, doileur. - R. we Plessas. - Magnelane, capilaine d'artillerie, commandant la place. - Bentasive, Bolor. docleurs. - Alvenvie, curé do Laghoual. - I)Lsond, vieaire. - Colonec, receveur. - Gunacd, oflicier d'administration. - Gesano, officier d'adnunistration des hópıtaux. - Pulcenent, lueutenant, commandant le poste de Djelfa. - Dinst's, lieitenant adjout 8 . - Secineras, capilaine du génie. - Bucderas, interprele militaire. - Husanar, velerioaire. - Mouls, heutenant adjo:nt. - Prassies, pharmacien. - Alray, lieutenant. - Mecviem, garde du genie. - MaAma: - Bex-Dullasi, lieutenant de spahis. - Canacs, rapilaue, clef du bureau arabo. - Cusins Au, agha de Laghouat.

(1) M. Ie lieuteant Dinst's devint ensuite chef de Pannese de Djelfa et

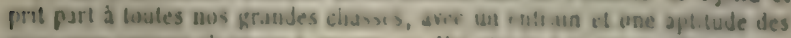

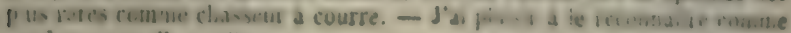
un de mes meilleurs éleves. 



\section{T.IBLE}

Pases

Denciace de te premiere sdition.............. 3

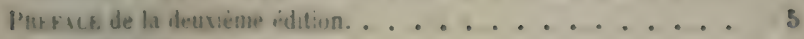

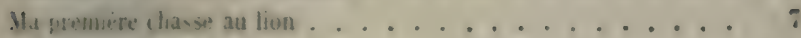

Ml prentere planthiere. . . . . . . . . . . . . . 3i

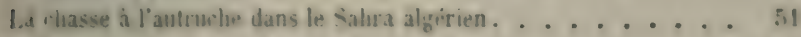

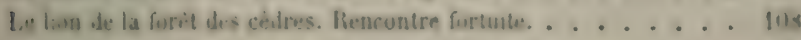

l.e livur de la moniligne et son parte ave un lon. . . . . . 11:3

La chawe au frmen . . . . . . . . . . . . 135

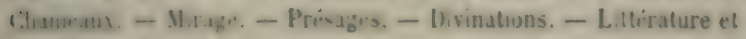

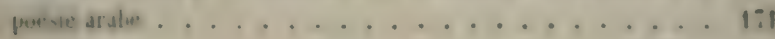

Clisturau. ...................... 1:1

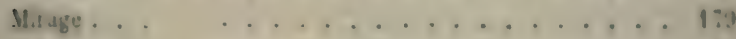

Irisapt...................... 181

Divinatiuns. . . . . . . . . . . . 14:3

Liturature et poisice arabe de nos jours. . . . . . . . 18.

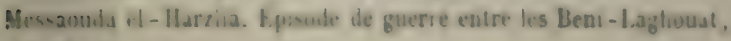

les Lar bas el Kar - el- llirine ............... 196

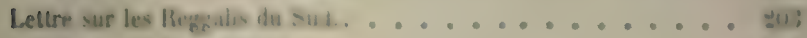

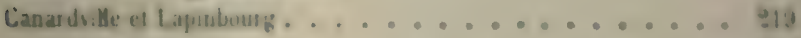

Canaril le...................... 919

Lapint . . . . . . . . . . . . . 803

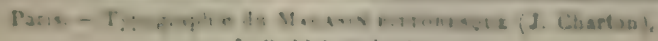

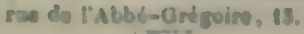





SK

255

A6M3

1888

BioMed
Margueritte, Auguste,

Chasses de I'Algérie. 4. éd.

SLIPS FROM THIS POCKET

\section{UNIVERSITY OF TORONTO LIBRARY}


(X)

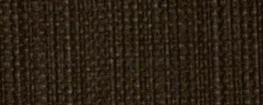

\begin{tabular}{|c|c|c|}
\hline & &
\end{tabular} 